\title{
Differential Dihydrofunctionalization of Terminal Alkynes: Synthesis of Benzylic Alkyl Boronates Through Reductive Three- Component Coupling
}

Megan K. Armstrong and Gojko Lalic*

Department of Chemistry, University of Washington, Seattle, Washington 98195, United States 


\section{Table of Contents}

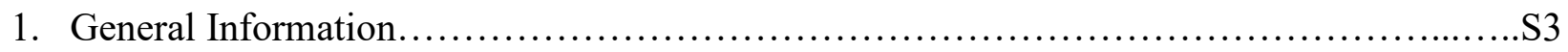

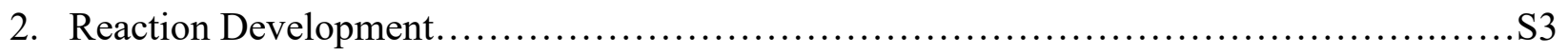

3. General Procedure for Differential Dihydrofunctionalization of Alkynes...................S7

4. Characterization of Product of Differential Dihydrofunctionalization....................S8

5. Aryl Bromide Starting Materials...................................................................................... 19

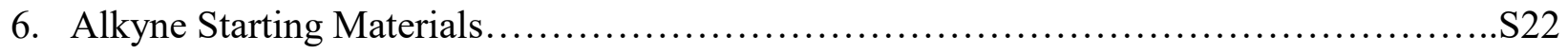

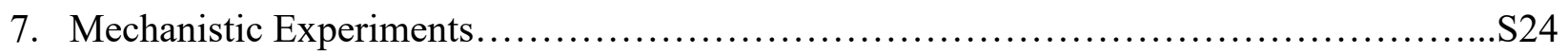

Analysis of Potential Catalytic Intermediates....................................S24

Synthesis of Heterobimetallic Complex.......................................... 25

Palladium-Catalyzed Cross Coupling of Heterobimetallic Complex and ArBr..........S26

Evaluation of Catalytic Intermediates in Catalytic Reaction.........................S27

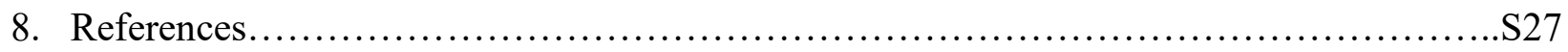

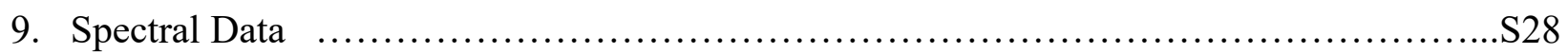




\section{General Information}

All reactions were performed under a nitrogen atmosphere with flame-dried or oven-dried (120 ${ }^{\circ} \mathrm{C}$ ) glassware, using standard Schlenk techniques, or in a glovebox (Nexus II from Vacuum Atmospheres). Column chromatography was performed using a Biotage Iso-1SV flash purification

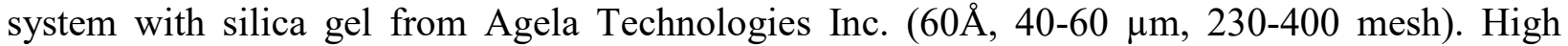
Pressure Liquid Chromatography was performed using a Agilent LC column (Zorbax CN PrepHT, $21.2 \times 250 \mathrm{~mm}, 7 \mu \mathrm{m}$ ). Infrared (IR) spectra were recorded on a Perkin Elmer Spectrum RX I spectrometer. IR peak absorbencies are represented as follows: $\mathrm{s}=$ strong, $\mathrm{m}=$ medium, $\mathrm{w}=\mathrm{weak}$, br $=$ broad. ${ }^{1} \mathrm{H}$ - and ${ }^{13} \mathrm{C}$ NMR spectra were recorded on a Bruker AV-300 or AV-500 spectrometer. ${ }^{1} \mathrm{H}$ NMR chemical shifts $(\delta)$ are reported in parts per million $(\mathrm{ppm})$ downfield of TMS and are referenced relative to residual solvent peak $\left(\mathrm{CDCl}_{3}: \delta 7.26 \mathrm{ppm}\right) .{ }^{13} \mathrm{C}$ NMR chemical shifts are reported in parts per million downfield of TMS and are referenced to the carbon resonance of the solvent $\left(\mathrm{CDCl}_{3}: \delta 77.2 \mathrm{ppm}\right) .{ }^{19} \mathrm{~F}$ NMR chemical shifts $(\delta)$ are reported in parts per million (ppm) and are referenced relative to the internal standard, hexafluorobenzene $\left(\mathrm{C}_{6} \mathrm{~F}_{6}: \delta-164.9 \mathrm{ppm}\right) .{ }^{11} \mathrm{~B}$ NMR chemical shifts $(\delta)$ are reported in part per million $(\mathrm{ppm})$. Data are represented as follows: chemical shift, multiplicity $(\mathrm{s}=$ singlet, $\mathrm{d}=$ doublet, $\mathrm{t}=$ triplet, $\mathrm{q}=$ quartet, $\mathrm{p}=$ pentet, hept $=$ heptet, $\mathrm{m}=$ multiplet), coupling constants in Hertz $(\mathrm{Hz})$, intergration. Mass spectra were collected on a JEOL HX-110 mass spectrometer. GC analysis was performed on a Shimadzu GC-2010 instrument with a flame ionization detector and a SHRXI-5MS column $(15 \mathrm{~m}, 0.25 \mathrm{~mm}$ inner diameter, $0.25 \mu \mathrm{m}$ film thickness). The following temperature program was used: $2 \mathrm{~min} @ 60{ }^{\circ} \mathrm{C}$, $13{ }^{\circ} \mathrm{C} / \mathrm{min}$ to $160{ }^{\circ} \mathrm{C}, 30{ }^{\circ} \mathrm{C} / \mathrm{min}$ to $250{ }^{\circ} \mathrm{C}, 5.5 \mathrm{~min} @ 250{ }^{\circ} \mathrm{C}$.

Materials: THF, $\mathrm{CH}_{2} \mathrm{Cl}_{2}$, ether, benzene, and toluene were degassed and dried by passing through columns of neutral alumina. Anhydrous isooctane was purchased from Millipore Sigma, and was subsequently degassed and stored over $4 \AA$ molecular sieves. Pinacolborane was purchase from TCI America and distilled over calcium hydride under reduced pressure before use. Deuterated solvents were purchased from Cambridge Isotope Laboratories, Inc. and were stored over $4 \AA$ molecular sieves prior to use. Commercial reagents were purchased from Millipore Sigma, TCI America, GFS-Chemicals, Ark-Pharm, Combi-Blocks, Oakwood Chemicals, Strem Chemicals and Alfa Aesar.

\section{Reaction Development (Table 1)}

All reactions were performed on a $0.05 \mathrm{mmol}$ scale with the stoichiometry shown in Tables S1S9. In a nitrogen-filled glovebox a dram vial was charged with a stir bar, alkoxide additive (Table S1), 5-phenyl-1-pentyne, 1,3,5-trimethoxybenzene (TMB, used as an internal standard for GC), palladium (Table S2 and S7), IPrCuX (Table S3 an S6), ligand (Table S4), 4-bromoanisole, HBpin (Table S5) and toluene (Table S8 and S9). The reaction mixture was stirred at $45^{\circ} \mathrm{C} .30 \mu \mathrm{L}$ aliquots were taken at $6 \mathrm{~h}$, and $24 \mathrm{~h}$ time points, pushed through a plug of silica with $1.5 \mathrm{~mL}$ of EtOAc and monitored by Gas Chromatography.

During preliminary reaction optimization both aryl chlorides and aryl bromides were explored. Aryl bromides were found to be competent coupling partners, while aryl chlorides did not provide product and were not used in further reaction development. 
Table S1: Alkoxide Additive Screen

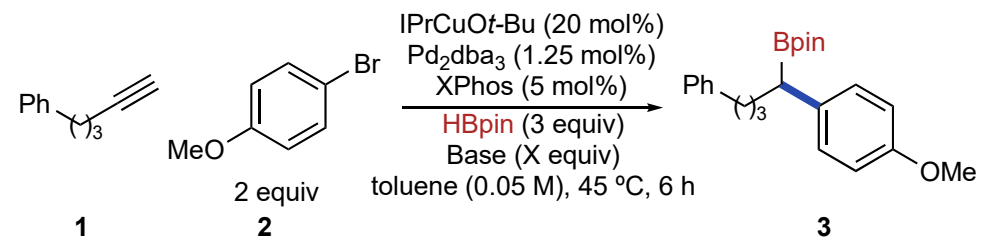

\begin{tabular}{ccccc} 
Entry & Alkoxide & Stoichiometry & \%Yield $6 \mathrm{~h}$ & \%Yield $24 \mathrm{~h}$ \\
\hline 1 & $\mathrm{NaO} t-\mathrm{Bu}$ & 2 equiv & 52 & 53 \\
2 & $\mathrm{NaO} t-\mathrm{Bu}$ & 3 equiv & 54 & 56 \\
3 & $\mathrm{LiO} t-\mathrm{Bu}$ & 2 equiv & 18 & 20 \\
4 & $\mathrm{LiO} t-\mathrm{Bu}$ & 3 equiv & 34 & 36 \\
5 & $\mathrm{KO} t-\mathrm{Bu}$ & 1 equiv & 5 & 4 \\
6 & $\mathrm{KO} t$-Bu & 2 equiv & 38 & 40 \\
7 & $\mathrm{KO} t-\mathrm{Bu}$ & 2.5 equiv & 82 & 85 \\
8 & $\mathrm{KO} t$-Bu & 3 equiv & 86 & 87 \\
9 & $\mathrm{KO} t$-Bu & 4 equiv & 73 & 75 \\
10 & $\mathrm{KO} t$-Bu & 5 equiv & 60 & 62
\end{tabular}

Table S2: Palladium Catalyst Screen

\begin{tabular}{|c|c|c|c|}
\hline Pd sourc & $\underbrace{\mathrm{IPrC}}_{2 \text { equiv }}$ & $\begin{array}{l}\mathrm{Bu}(20 \mathrm{~mol} \%) \\
\frac{(1.25 \mathrm{~mol} \%)}{(5 \mathrm{~mol} \%)} \\
\frac{(3 \text { equiv) }}{\text { ( (2 equiv) }} \\
55 \mathrm{M}), 45^{\circ} \mathrm{C}, 6 \mathrm{~h}\end{array}$ & Bpin \\
\hline Entry & Pd Source & $\%$ Yield $6 \mathrm{~h}$ & $\%$ Yield $24 \mathrm{~h}$ \\
\hline 1 & $\mathrm{Pd}(\mathrm{OAc})_{2}$ & 54 & 56 \\
\hline 2 & $\mathrm{Pd}_{2} \mathrm{dba}_{3}$ & 86 & 87 \\
\hline 3 & $\mathrm{Pddba}_{2}$ & 39 & 42 \\
\hline 4 & $\operatorname{Pd}\left(t-\mathrm{Bu}_{3}\right)_{2}$ & 20 & 30 \\
\hline 5 & $\mathrm{Pd}\left(\mathrm{PPh}_{3}\right)_{2} \mathrm{Cl}_{2}$ & 3 & 4 \\
\hline 6 & $\operatorname{Pd}(\mathrm{TFA})_{2}$ & 35 & 34 \\
\hline 7 & Peppsi Pd & 32 & 33 \\
\hline 8 & $\mathrm{Pd}($ cinnamyl $) \mathrm{Cl}_{2}$ & 15 & 16 \\
\hline 9 & $\mathrm{Pd}(\mathrm{COD})_{2} \mathrm{Cl}_{2}$ & 21 & 31 \\
\hline
\end{tabular}

Table S3: Copper Catalyst Screen

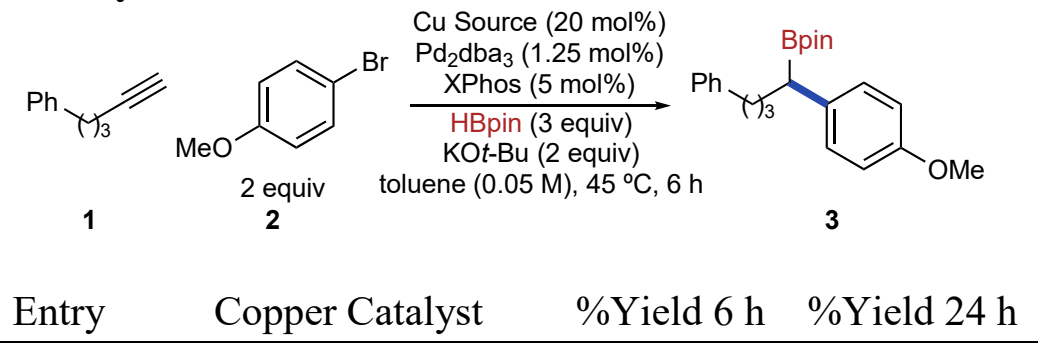




\begin{tabular}{cccc}
\hline 1 & $\mathrm{IPrCuOt}-\mathrm{Bu}$ & 86 & 87 \\
2 & $\mathrm{IPrCuCl}$ & 67 & 70 \\
3 & $\mathrm{SIPrCuCl}$ & 58 & 60 \\
4 & $\mathrm{SIPrCuO} t-\mathrm{Bu}$ & 54 & 58 \\
5 & $\mathrm{IMesCuCl}$ & 8 & 10 \\
6 & $\mathrm{SIMesCuCl}$ & 10 & 11 \\
7 & $\mathrm{ICyCuCl}$ & 0 & 0 \\
8 & $\mathrm{I} t-\mathrm{BuCuCl}$ & 0 & 1 \\
9 & $\mathrm{IBoxCuCl}$ & 0 & 0 \\
10 & $\mathrm{IPr}{ }^{*} \mathrm{CuCl}$ & 4 & 7 \\
11 & $(6 \mathrm{DIPP}) \mathrm{CuO} t-\mathrm{Bu}$ & 3 & 3 \\
12 & $\mathrm{CyIMesCuCl}$ & 0 & 0 \\
13 & $\mathrm{CyIPrCuCl}$ & 3 & 4 \\
14 & $\mathrm{CyIBoxCuCl}$ & 1 & 2 \\
15 & $\mathrm{IPrCuO}\left(2-t-\mathrm{Bu}-\mathrm{C}_{6} \mathrm{H}_{4}\right)$ & 43 & 47
\end{tabular}

Table S4: Ligand Screen

\begin{tabular}{|c|c|c|c|}
\hline 1 & $\begin{array}{r}\mathrm{IPrCuOt}-\mathrm{B} \\
\mathrm{Br} \\
\mathrm{Pd}_{2} \mathrm{dba}_{3}( \\
\text { ligand ( } \\
\mathrm{HBpin} \\
\mathrm{KOt} \text {-Bu } \\
\text { toluene }(0.05\end{array}$ & $\begin{array}{l}20 \mathrm{~mol} \%) \\
25 \mathrm{~mol} \%) \\
\text { mol\%) } \\
\underset{\text { equiv) }}{\longrightarrow} \\
\text { equiv) } \\
\text { l), } 45^{\circ} \mathrm{Ch}, 6 \mathrm{~h}\end{array}$ & Bpin \\
\hline Entry & Ligand & $\%$ Yield $6 \mathrm{~h}$ & $\%$ Yield $24 \mathrm{~h}$ \\
\hline 1 & XPhos & 86 & 87 \\
\hline 2 & RuPhos & 18 & 18 \\
\hline 3 & SPhos & 6 & 8 \\
\hline 4 & BrettPhos & 20 & 22 \\
\hline 5 & DavePhos & 13 & 16 \\
\hline 6 & CPhos & 8 & 15 \\
\hline 7 & Di-BIME & 3 & 5 \\
\hline 8 & JosiPhos & 4 & 4 \\
\hline 9 & Me-DuPhos & 2 & 2 \\
\hline 10 & $\mathrm{PCy}_{3}$ & 5 & 17 \\
\hline 11 & $(R)$-DTBM-SEGPhos & 8 & 8 \\
\hline 12 & QuinoxP & 2 & 5 \\
\hline 13 & XantPhos & 0 & 0 \\
\hline 14 & chiraphos & 5 & 6 \\
\hline 15 & $r a c$-BINAP & 5 & 5 \\
\hline 16 & NMDPP & 2 & 3 \\
\hline 17 & DPPF & 7 & 7 \\
\hline
\end{tabular}


Table S5: HBpin Stoichiometry Screen

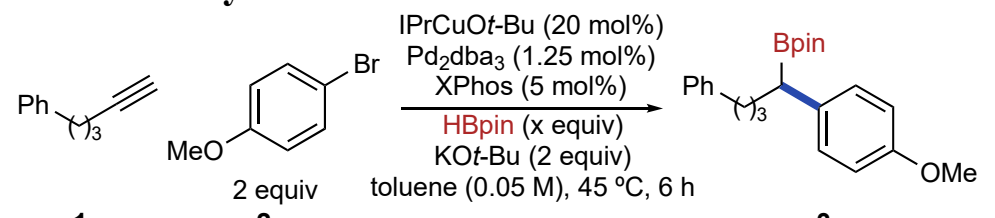

1

2

3

\begin{tabular}{cccr} 
Entry & Equivalents of HBpin & \%Yield 6 h & \%Yield \\
\hline 1 & 2.0 & 49 & 50 \\
2 & 2.5 & 61 & 60 \\
3 & 2.8 & 71 & 74 \\
4 & 3.0 & 86 & 87 \\
5 & 4.0 & 55 & 55 \\
6 & 5.0 & 52 & 53
\end{tabular}

Table S6: Copper Catalyst Loading Screen

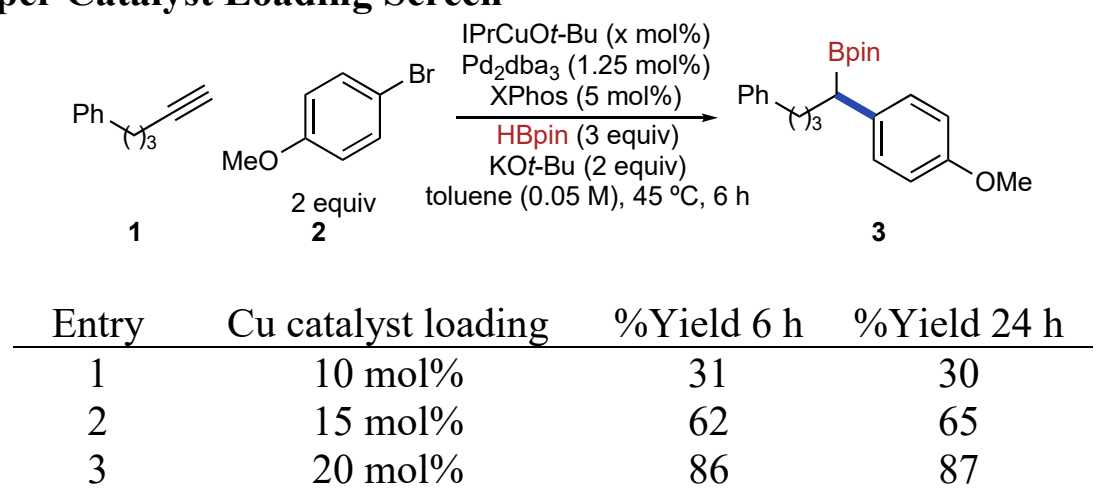

Table S7: Palladium Catalyst Loading Screen

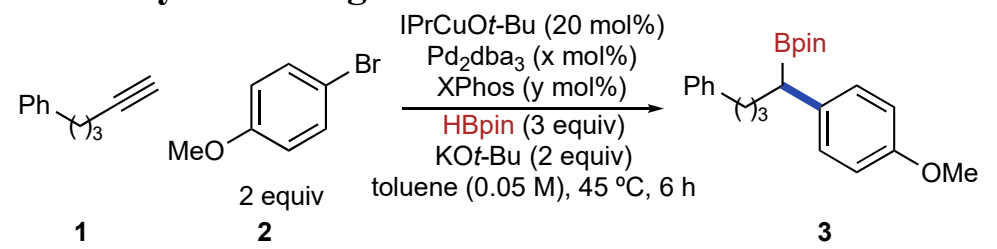

\begin{tabular}{ccccc} 
Entry & Pd catalyst loading & XPhos Loading & \%Yield 6 & \%Yield 24 h \\
\hline 1 & $1.25 \mathrm{~mol} \%$ & $5 \mathrm{~mol} \%$ & 86 & 87 \\
2 & $2.5 \mathrm{~mol} \%$ & $10 \mathrm{~mol} \%$ & 59 & 60 \\
3 & $5 \mathrm{~mol} \%$ & $20 \mathrm{~mol} \%$ & 32 & 32
\end{tabular}

Table S8: Solvent Screen

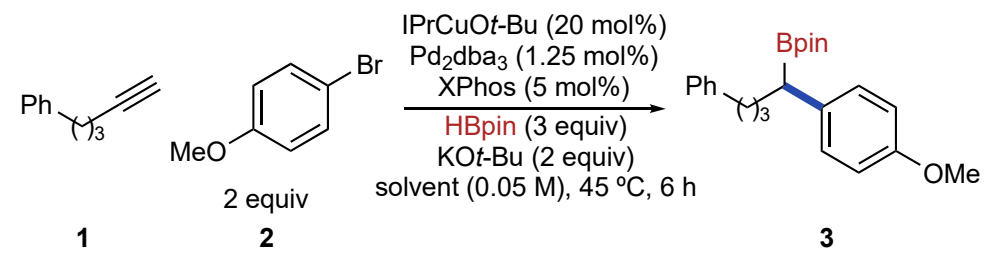




\begin{tabular}{cccc} 
Entry & Solvent & \%Yield 6 h & \%Yield 24 h \\
\hline 1 & toluene & 86 & 87 \\
2 & benzene & 65 & 68 \\
3 & isooctane & 24 & 24 \\
4 & dioxane & 5 & 6 \\
5 & THF & 0 & 0 \\
6 & ether & 0 & 0 \\
7 & DCM & 0 & 0 \\
8 & DME & 0 & 0
\end{tabular}

Table S9: Limiting Reagent Concentration Screen

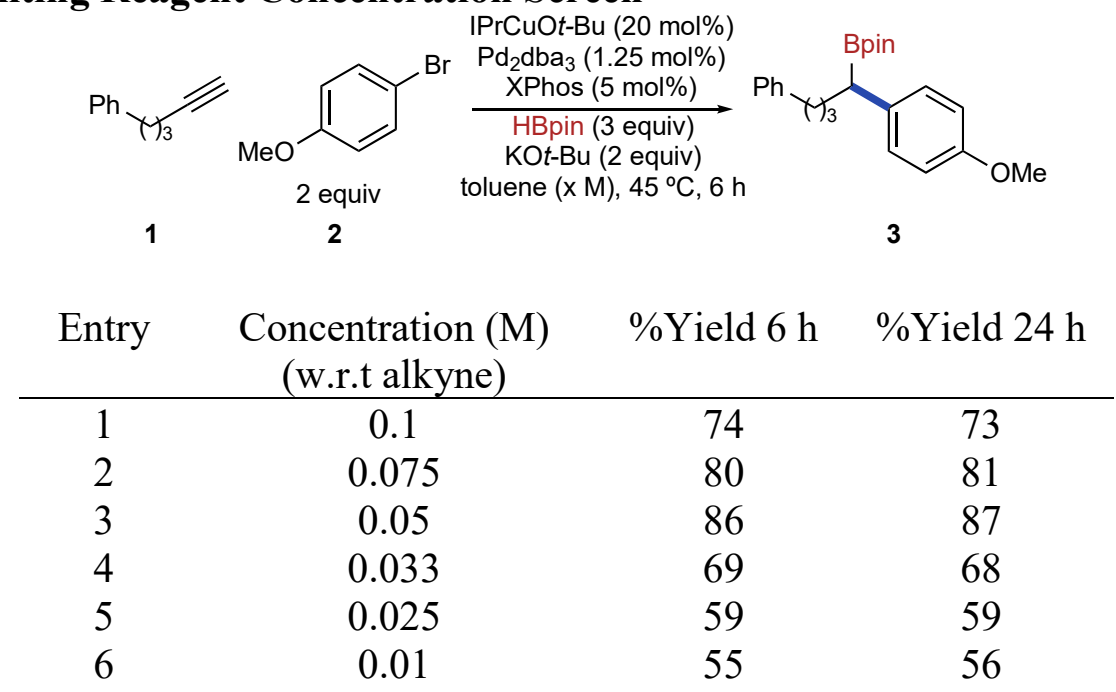

3. General Procedure for the Differential Dihydrofunctionalization of Alkynes (Table 2)

In a nitrogen-filled glovebox, a scintillation vial was charged with a stir bar, $\mathrm{KO} t$ - $\mathrm{Bu}(122.3 \mathrm{mg}$, $1.00 \mathrm{mmol}, 2.0$ equiv), $\mathrm{IPrCuOt}$-Bu (52.6 mg, $0.1 \mathrm{mmol}, 0.20$ equiv), HBpin (192.0 mg, 1.50 mmol, 3.0 equiv), toluene $(10 \mathrm{~mL}, 0.05 \mathrm{M})$ and alkyne $(0.5 \mathrm{mmol}, 1.0$ equiv). The reaction mixture was stirred at $45^{\circ} \mathrm{C}$ until the yellow color disappeared. To this reaction mixture was added $\mathrm{Pd}_{2} \mathrm{dba}_{3}$ (5.7 mg, $0.00625 \mathrm{mmol}, 0.0125$ equiv), XPhos ( $11.9 \mathrm{mg}, 0.025$ equiv) and aryl bromide (1.0 mmol, 2.0 equiv) and the reaction mixture was vigorously stirred at $45^{\circ} \mathrm{C}$. After $6 \mathrm{~h}$ a $60 \mu \mathrm{L}$ was taken and pushed through a plug of silica with $1.5 \mathrm{~mL}$ EtOAc and analyzed by GC. Upon consumption of the alkyne, the reaction mixture was diluted with $\mathrm{Et}_{2} \mathrm{O}$, washed with $1 \mathrm{M} \mathrm{HCl}$ and brine, dried over $\mathrm{Na}_{2} \mathrm{SO}_{4}$, filtered through a pad a silica gel and concentrated under reduced pressure. The crude mixture was purified by silica gel column chromatography.

A variety of aryl chlorides were also surveyed under the optimized reaction conditions using 5phenyl-1-pentyne as a coupling partner, but did not produce the desired product and were not further optimized. 


\section{Characterization of Differential Dihydrofunctionalization Products}<smiles>COc1ccc(C(CCCCc2ccccc2)c2ccccc2)cc1</smiles>

2-[1-(4-methoxyphenyl)-5-phenylpentyl]-4,4,5,5-tetramethyl-1,3,2-dioxaborolane compound was prepared according to reported procedure and was purified by silica gel column chromatography, 0-10\% EtOAc in hexanes and was isolated as a clear colorless liquid (162 mg, 85\% yield). ${ }^{1} \mathrm{H}$ NMR (300 MHz, Chloroform- $d$ ) $\delta 7.28-7.23(\mathrm{~m}, 3 \mathrm{H}), 7.21-7.06(\mathrm{~m}, 5 \mathrm{H}), 6.80$ $(\mathrm{d}, J=8.6 \mathrm{~Hz}, 2 \mathrm{H}), 3.78(\mathrm{~s}, 3 \mathrm{H}), 2.57(\mathrm{t}, J=7.7 \mathrm{~Hz}, 2 \mathrm{H}), 2.24(\mathrm{t}, J=7.0 \mathrm{~Hz}, 1 \mathrm{H}), 1.95-1.73(\mathrm{~m}$, $1 \mathrm{H}), 1.72-1.51(\mathrm{~m}, 4 \mathrm{H}), 1.38-1.24(\mathrm{~m}, 3 \mathrm{H}), 1.18(\mathrm{~d}, J=7.3 \mathrm{~Hz}, 12 \mathrm{H}) .{ }^{13} \mathrm{C} \mathrm{NMR}(126 \mathrm{MHz}$, Chloroform- $d$ ) $\delta 157.4,142.9,135.5,129.3,128.5,128.3,125.6,113.9,83.3,55.2,35.9$, 32.9, 31.5, 29.0, 25.0, 24.7, 24.7. ${ }^{11} \mathrm{~B}$ NMR (96 MHz, Chloroform- $d$ ) $\delta$ 32.7. GCMS (EI) calculated for $[\mathrm{M}]+380.25$, found 380.3. FTIR (neat, $\left.\mathrm{cm}^{-1}\right): 3053(\mathrm{~m}), 2980(\mathrm{~s}), 2929(\mathrm{~s}), 2858(\mathrm{~m}), 1604(\mathrm{~m})$, 1510(s), 1456(m), 1371(m), 1325(m), 1246(m), 1145(s), 1030(m), 851(m), 755(s).<smiles>FC(F)(F)c1ccc(C(CCCCc2ccccc2)c2ccccc2)cc1</smiles>

\section{4,4,5,5-tetramethyl-2-\{5-phenyl-1-[4-(trifluoromethyl)phenyl]pentyl\}-1,3,2-dioxaborolane}

(8), compound was prepared according to reported procedure using IPrCuCl instead of IPrCuOt$\mathrm{Bu}$ and $\mathrm{NaO} t$ - $\mathrm{Bu}$ instead of $\mathrm{KO} t$ - $\mathrm{Bu}$, and was purified by silica gel chromatography, $0-100 \% \mathrm{DCM}$ in hexanes and was isolated as a clear colorless liquid (171 mg, 82\% yield). ${ }^{1} \mathrm{H} \mathrm{NMR} \mathrm{(300} \mathrm{MHz,}$ Chloroform- $d$ ) $\delta 7.49(\mathrm{~d}, J=8.0 \mathrm{~Hz}, 2 \mathrm{H}), 7.34-7.19(\mathrm{~m}, 6 \mathrm{H}), 7.21-7.09(\mathrm{~m}, 3 \mathrm{H}), 2.65-2.51$ $(\mathrm{m}, 2 \mathrm{H}), 2.36(\mathrm{t}, J=7.9 \mathrm{~Hz}, 1 \mathrm{H}), 1.97-1.79(\mathrm{~m}, 1 \mathrm{H}), 1.76-1.51(\mathrm{~m}, 3 \mathrm{H}), 1.39-1.22(\mathrm{~m}, 2 \mathrm{H})$, $1.18(\mathrm{~d}, J=5.7 \mathrm{~Hz}, 12 \mathrm{H}) .{ }^{13} \mathrm{C}$ NMR $(126 \mathrm{MHz}$, Chloroform- $d) \delta 147.9,142.7,128.7,128.5,128.3$, $127.3(\mathrm{q}, J=34.2 \mathrm{~Hz}), 125.7,125.3(\mathrm{q}, J=3.7 \mathrm{~Hz}), 124.8(\mathrm{q}, J=266.7 \mathrm{~Hz}), 83.6,35.8,32.4,31.4$, 28.9, 24.7, 24.7. ${ }^{19} \mathrm{~F}$ NMR (470 MHz, Chloroform- $d$ ) $\delta-65.1 .{ }^{11} \mathrm{~B}$ NMR (96 MHz, Chloroform- $d$ ) $\delta$ 32.5. GCMS (EI) calculated for $[\mathrm{M}]+418.23$, found 418.4. FTIR (neat, $\left.\mathrm{cm}^{-1}\right): 3027(\mathrm{~m}), 2979(\mathrm{~s})$, 2931(s), 2858(m), 1617(s), 1454(m), 1371(s), 1325(s), 1164(m), 1123(s), 1068(s), 1018(s), 966(m), 851(m), 734(m), 699(m).<smiles>Fc1ccc(C(Br)CCCCc2ccccc2)cc1</smiles>

2-[1-(4-fluorophenyl)-5-phenylpentyl]-4,4,5,5-tetramethyl-1,3,2-dioxaborolane

(9), compound was prepared according to reported procedure and was purified by silica gel chromatography, $0-100 \%$ DCM in hexanes and was isolated as a clear colorless liquid (172 mg, 93\% yield). ${ }^{1} \mathrm{H}$ NMR (300 MHz, Chloroform- $d$ ) $\delta 7.32-7.10(\mathrm{~m}, 8 \mathrm{H}), 6.93(\mathrm{t}, J=8.8 \mathrm{~Hz}, 2 \mathrm{H})$, $2.65-2.50(\mathrm{~m}, 1 \mathrm{H}), 2.27(\mathrm{t}, J=7.9 \mathrm{~Hz}, 1 \mathrm{H}), 1.93-1.76(\mathrm{~m}, 1 \mathrm{H}), 1.73-1.46(\mathrm{~m}, 3 \mathrm{H}), 1.38-1.10$ $(\mathrm{m}, 14 \mathrm{H}) .{ }^{13} \mathrm{C}$ NMR $(126 \mathrm{MHz}$, Chloroform- $d$ ) $\delta 161.1(\mathrm{~d}, J=242.3 \mathrm{~Hz}), 142.8,139.1,129.7(\mathrm{~d}$, $J=7.9 \mathrm{~Hz}), 128.4(\mathrm{~d}, J=24.0 \mathrm{~Hz}), 125.7,115.0(\mathrm{~d}, J=20.9 \mathrm{~Hz}), 83.4,35.9,32.7,31.4,28.9,24.9$, 24.7, 24.7. ${ }^{19} \mathrm{~F}$ NMR (470 MHz, Chloroform- $d$ ) $\delta$-121.7. ${ }^{11} \mathrm{~B}$ NMR (96 MHz, Chloroform- $d$ ) $\delta$ 
32.4. GCMS (EI) calculated for [M]+ 368.23, found 368.3. FTIR (neat, $\left.\mathrm{cm}^{-1}\right)$ : 3058(m), 2978(s), 2930(s), 2857(m), 1603(s), 1506(s), 1370(s), 1324(s), 1219(s), 1142(s), 967(m), 852(m), 699(m).<smiles>Clc1ccc(C(Br)CCCCc2ccccc2)cc1</smiles>

2-[1-(4-chlorophenyl)-5-phenylpentyl]-4,4,5,5-tetramethyl-1,3,2-dioxaborolane

(10), compound was prepared according to reported procedure and was purified by silica gel chromatography $0-100 \%$ DCM in hexanes, and was isolated as a clear colorless liquid (126 mg, $65 \%$ yield). ${ }^{1} \mathrm{H}$ NMR (300 MHz, Chloroform- $d$ ) $\delta 7.31-7.06(\mathrm{~m}, 11 \mathrm{H}), 2.65-2.50(\mathrm{~m}, 2 \mathrm{H}), 2.26$ $(\mathrm{t}, J=7.9 \mathrm{~Hz}, 1 \mathrm{H}), 1.94-1.75(\mathrm{~m}, 1 \mathrm{H}), 1.60(\mathrm{~m}, 3 \mathrm{H}), 1.31(\mathrm{~m}, 2 \mathrm{H}), 1.17(\mathrm{~d}, J=6.0 \mathrm{~Hz}, 12 \mathrm{H}) .{ }^{13} \mathrm{C}$ NMR (126 MHz, Chloroform- $d$ ) $\delta$ 142.8, 142.1, 130.9, 129.8, 128.5, 128.3, 125.7, 120.1, 83.5, 35.9, 32.5, 31.4, 28.9, 24.8, 24.7, 24.7. ${ }^{11} \mathrm{~B}$ NMR (96 MHz, Chloroform- $d$ ) $\delta$ 32.3. GCMS (EI) calculated for $[\mathrm{M}]+384.20$, found 384.3. FTIR (neat, $\left.\mathrm{cm}^{-1}\right)$ : 3059(m), 2979(s), 2931(s), 2858(m), 1604(s), 1490(s), 1366(s), 1322(s), 1143(s), 1091(s), 1015(s), 967(m), 851(m), 699(m).<smiles>CN(C)c1ccc(C(CCCCc2ccccc2)Cc2ccccc2)cc1</smiles>

$N, N$-dimethyl-4-[5-phenyl-1-(4,4,5,5-tetramethyl-1,3,2-dioxaborolan-2-yl)pentyl]aniline (11), compound was prepared according to reported procedure using $\mathrm{IPrCuCl}$ instead of $\mathrm{IPrCuO} t-\mathrm{Bu}$, $\mathrm{NaO} t-\mathrm{Bu}$ instead of $\mathrm{KO} t-\mathrm{Bu}$ and a 1:1 mixture of toluene isooctane, and was purified by prep TLC, $2 \%$ TEA, $20 \%$ EtOAc in hexanes, and was isolated as a clear colorless liquid (156 mg, 79\% yield). ${ }^{1} \mathrm{H}$ NMR $(300 \mathrm{MHz}$, Chloroform- $d$ ) $\delta 7.83(\mathrm{~d}, J=9.0 \mathrm{~Hz}, 2 \mathrm{H}), 7.27-7.19(\mathrm{~m}, 6 \mathrm{H}), 6.61(\mathrm{~d}, J=$ $9.0 \mathrm{~Hz}, 2 \mathrm{H}), 3.01$ (s, 6H), 2.86 (t, $J=7.1 \mathrm{~Hz}, 2 \mathrm{H}), 2.63$ (t, $J=7.3 \mathrm{~Hz}, 2 \mathrm{H}), 1.88-1.83(\mathrm{~m}, 1 \mathrm{H})$, $1.70-1.55(\mathrm{~m}, 3 \mathrm{H}), 1.35-1.16(\mathrm{~m}, 14 \mathrm{H}) .{ }^{13} \mathrm{C}$ NMR $(126 \mathrm{MHz}$, Chloroform- $d$ ) $\delta 153.4,142.6$, $130.4,130.4,128.5,128.4,125.8,125.2,110.8,83.5,40.1,37.8,36.0,31.4,24.9,24.7,24.7 .{ }^{11} \mathrm{~B}$ NMR (96 MHz, Chloroform- $d$ ) $\delta$ 32.8. GCMS (EI) calculated for [M]+ 393.28, found 393.3. FTIR (neat, $\left.\mathrm{cm}^{-1}\right):$ 2052(m), 2980(s), 2940(s), 2858(m), 1606(m), 1511(s), 1456(m), 1415(m), 1340(m), 1248(m), 1152(s), 1035(m), 850(m), 755(s), 699(m).<smiles>N#Cc1ccc(C(CCCCc2ccccc2)c2ccccc2)cc1</smiles>

4-[5-phenyl-1-(4,4,5,5-tetramethyl-1,3,2-dioxaborolan-2-yl)pentyl]benzonitrile (12), compound was prepared according to reported procedure using $\mathrm{IPrCuCl}$ instead of $\mathrm{IPrCuO} t-\mathrm{Bu}$ and $\mathrm{NaO} t-\mathrm{Bu}$ instead of $\mathrm{KO} t-\mathrm{Bu}$, and was purified by silica gel column chromatography, $0-10 \%$ EtOAc in hexanes and was isolated as a clear colorless liquid (120 mg, 64\% yield). ${ }^{1} \mathrm{H}$ NMR (300 MHz, Chloroform- $d$ ) $\delta 7.53(\mathrm{~d}, J=8.3 \mathrm{~Hz}, 2 \mathrm{H}), 7.34-7.21(\mathrm{~m}, 5 \mathrm{H}), 7.15(\mathrm{~m}, 3 \mathrm{H}), 2.57(\mathrm{td}, J=$ 7.4, 3.4 Hz, 2H), 2.37 (t, $J=7.9 \mathrm{~Hz}, 1 \mathrm{H}), 1.98-1.81(\mathrm{~m}, 1 \mathrm{H}), 1.75-1.55(\mathrm{~m}, 3 \mathrm{H}), 1.39-1.24$ $(\mathrm{m}, 4 \mathrm{H}), 1.17(\mathrm{~d}, J=5.0 \mathrm{~Hz}, 12 \mathrm{H}) .{ }^{13} \mathrm{C}$ NMR $(126 \mathrm{MHz}$, Chloroform- $d$ ) $\delta$ 149.6, 142.6, 132.2, $129.1,128.5,128.3,125.7,119.5,109.0,83.8,35.8,32.0,31.3,28.8,25.0,24.7,24.7 .{ }^{11} \mathrm{~B}$ NMR $(96 \mathrm{MHz}$, Chloroform- $d$ ) $\delta$ 32.3. GCMS (EI) calculated for $[\mathrm{M}]+375.23$, found 375.2. FTIR (neat, 
$\left.\mathrm{cm}^{-1}\right): 3049(\mathrm{~m}), 2978(\mathrm{~s}), 2931(\mathrm{~s}), 2857(\mathrm{~m}), 2226(\mathrm{~s}), 1605(\mathrm{~s}), 1455(\mathrm{~m}), 1370(\mathrm{~s}), 1327(\mathrm{~s}), 1142(\mathrm{~s})$, 967(m), 851(m), 699(m).

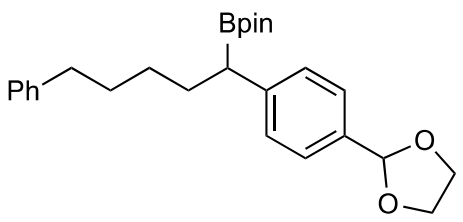

2-\{1-[4-(1,3-dioxolan-2-yl)phenyl]-5-phenylpentyl\}-4,4,5,5-tetramethyl-1,3,2-dioxaborolane (13), compound was prepared according to reported procedure and was purified by silica gel column chromatography 0-10\% EtOAc in hexanes and was isolated as a clear colorless liquid (187 mg, 89\% yield). ${ }^{1} \mathrm{H}$ NMR (300 MHz, Chloroform- $\left.d\right) \delta 7.36(\mathrm{~d}, J=8.1 \mathrm{~Hz}, 2 \mathrm{H}), 7.29-7.17$ (m, $5 \mathrm{H}), 7.14(\mathrm{~d}, J=8.1 \mathrm{~Hz}, 2 \mathrm{H}), 5.77(\mathrm{~s}, 1 \mathrm{H}), 4.29-3.88(\mathrm{~m}, 4 \mathrm{H}), 2.67-2.45(\mathrm{~m}, 2 \mathrm{H}), 2.30(\mathrm{t}, J=$ $7.9 \mathrm{~Hz}, 1 \mathrm{H}), 2.00-1.74(\mathrm{~m}, 1 \mathrm{H}), 1.74-1.46(\mathrm{~m}, 3 \mathrm{H}), 1.46-1.20(\mathrm{~m}, 2 \mathrm{H}), 1.16(\mathrm{~d}, J=6.7 \mathrm{~Hz}$, 12H). ${ }^{13} \mathrm{C}$ NMR (126 MHz, Chloroform- $d$ ) $\delta$ 144.7, 142.8, 134.5, 128.4, 128.4, 128.2, 126.5, 125.6, 104.0, 83.3, 65.4, 35.9, 32.5, 31.5, 28.9, 24.7, 24.7. ${ }^{11} \mathrm{~B}$ NMR (96 MHz, Chloroform- $d$ ) $\delta$ 32.6. GCMS (EI) calculated for [M]+ 422.26, found 422.3. FTIR (neat, $\left.\mathrm{cm}^{-1}\right): 3048(\mathrm{~m}), 2978(\mathrm{~s})$, 2930(s), 2857(s), 1615(s), 1454(m), 1370(m), 1325(s), 1217(m), 1217(m), 1142(m), 1020(m), 966(m), 911(w), 851(m), 699(m).

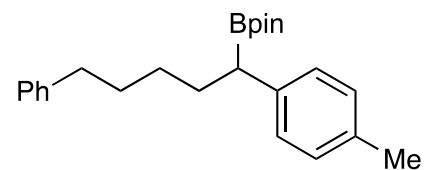

4,4,5,5-tetramethyl-2-[1-(4-methylphenyl)-5-phenylpentyl]-1,3,2-dioxaborolane

(14), compound was prepared according to reported procedure and was purified by silica gel chromatography, 0-100\% DCM in hexanes, and was isolated as a clear colorless liquid (142 mg, $78 \%$ yield). ${ }^{1} \mathrm{H}$ NMR (300 MHz, Chloroform- $d$ ) $\delta 7.34-7.01(\mathrm{~m}, 10 \mathrm{H}), 2.57(\mathrm{t}, J=9.0 \mathrm{~Hz}, 1 \mathrm{H})$, $2.43-2.20(\mathrm{~m}, 5 \mathrm{H}), 1.95-1.74(\mathrm{~m}, 1 \mathrm{H}), 1.64(\mathrm{dt}, J=15.2,7.6 \mathrm{~Hz}, 2 \mathrm{H}), 1.41-1.29(\mathrm{~m}, 2 \mathrm{H}), 1.18$ $(\mathrm{d}, J=7.2 \mathrm{~Hz}, 12 \mathrm{H}) .{ }^{13} \mathrm{C}$ NMR $(126 \mathrm{MHz}$, Chloroform-d) $\delta 142.8,140.3,134.4,129.3,129.0$, $128.4,128.3,125.6,83.2,35.9,32.7,31.5,29.0,24.7,24.6,24.6,21.1 .{ }^{11} \mathrm{~B}$ NMR (96 MHz, Chloroform- $d$ ) $\delta$ 32.3. GCMS (EI) calculated for $[\mathrm{M}]+364.26$, found 364.3. FTIR (neat, $\mathrm{cm}^{-1}$ ): 3059(m), 2977(m), 2857(m), 1604(s), 1512(m), 1453(m), 1370(m), 1325(m), 1142(s), 1030(m), 982(m), 851(m), 734(m).<smiles>Cc1cccc(C(CCCCc2ccccc2)Cc2ccccc2)c1</smiles>

4,4,5,5-tetramethyl-2-[1-(3-methylphenyl)-5-phenylpentyl]-1,3,2-dioxaborolane

(15), compound was prepared according to reported procedure and was purified by silica gel chromatography, $0-100 \%$ DCM in hexanes, and was isolated as a clear colorless liquid (134 mg, 74\% yield). ${ }^{1} \mathrm{H}$ NMR (300 MHz, Chloroform- $d$ ) $\delta 7.31-7.20(\mathrm{~m}, 3 \mathrm{H}), 7.21-7.08(\mathrm{~m}, 42 \mathrm{H}), 7.05$ $-6.90(\mathrm{~m}, 3 \mathrm{H}), 2.58(\mathrm{t}, J=7.8 \mathrm{~Hz}, 2 \mathrm{H}), 2.29(\mathrm{~d}, J=17.0 \mathrm{~Hz}, 4 \mathrm{H}), 1.97-1.76(\mathrm{~m}, 1 \mathrm{H}), 1.64(\mathrm{~m}$, $7.8 \mathrm{~Hz}, 3 \mathrm{H}), 1.35$ (t, $J=7.8 \mathrm{~Hz}, 2 \mathrm{H}), 1.18$ (d, $J=7.5 \mathrm{~Hz}, 12 \mathrm{H}) .{ }^{13} \mathrm{C}$ NMR (126 MHz, Chloroformd) $\delta 143.4,142.9,129.3,128.5,128.3,128.2,126.0,125.6,125.4,83.3,35.9,32.7,31.6,29.1$, 24.9, 24.7, 21.6. ${ }^{11} \mathrm{~B}$ NMR (96 MHz, Chloroform- $d$ ) $\delta$ 32.4. GCMS (EI) calculated for [M]+ 364.26, found 364.3. FTIR (neat, $\mathrm{cm}^{-1}$ ): 3061(m), 3026(m), 2978(s), 2929(s), 2857(m), 1604(s), 
1496(m), 1496(m), 1454(m), 1370(s), 1322(s), 1142(s), 1109(m), 967(m), 864(m), 750(m), 698(m).<smiles>COc1ccccc1C(Br)CCCCc1ccccc1</smiles>

2-[1-(2-methoxyphenyl)-5-phenylpentyl]-4,4,5,5-tetramethyl-1,3,2-dioxaborolane

(16), compound was prepared according to reported procedure using $\mathrm{IPrCuCl}$ instead of $\mathrm{IPrCuO} t-\mathrm{Bu}$ and $\mathrm{NaO} t$-Bu instead of $\mathrm{KO} t-\mathrm{Bu}$, and was purified by silica gel chromatography, $0-100 \% \mathrm{DCM}$ in hexanes, and was isolated as a clear colorless liquid (158 mg, 83\% yield). ${ }^{1} \mathrm{H} \mathrm{NMR} \mathrm{(300} \mathrm{MHz,}$ Chloroform- $d$ ) $\delta 7.36-7.05(\mathrm{~m}, 10 \mathrm{H}), 6.97-6.71(\mathrm{~m}, 2 \mathrm{H}), 3.78(\mathrm{~s}, 3 \mathrm{H}), 2.57(\mathrm{~m}, 2 \mathrm{H}), 2.42(\mathrm{t}, J$ $=7.7 \mathrm{~Hz}, 1 \mathrm{H}), 1.95-1.75(\mathrm{~m}, 1 \mathrm{H}), 1.75-1.55(\mathrm{~m}, 3 \mathrm{H}), 1.42-1.14(\mathrm{~m}, 14 \mathrm{H}) .{ }^{13} \mathrm{C}$ NMR $(126$ MHz, Chloroform- $d$ ) $\delta 157.1,143.1,132.4,129.7,128.5,128.2,126.3,125.5,120.6,110.0,83.1$, 55.1, 36.0, 31.6, 30.6, 29.0, 24.9, 24.7, 24.7. ${ }^{11} \mathrm{~B}$ NMR (96 MHz, Chloroform- $d$ ) $\delta$ 33.1. GCMS (EI) calculated for $[\mathrm{M}]+380.25$, found 380.3. FTIR (neat, $\left.\mathrm{cm}^{-1}\right): 3063(\mathrm{~s}), 2979(\mathrm{~m}), 2932(\mathrm{~s})$, 2858(m), 1600(m), 1490(m), 1454(m), 1370(s), 1319(s), 1241(m), 1144(m), 1030(m), 967(m), 851(m), 752(s).<smiles>c1ccc(CCCCC(Cc2ccccc2)c2ccc3ccccc3c2)cc1</smiles>

4,4,5,5-tetramethyl-2-[1-(naphthalen-2-yl)-5-phenylpentyl]-1,3,2-dioxaborolane

(17), compound was prepared according to reported procedure and was purified by silica gel chromatography, $0-100 \%$ DCM in hexanes, and was isolated as a clear colorless liquid (174 mg, $87 \%$ yield). ${ }^{1} \mathrm{H}$ NMR (300 MHz, Chloroform- $d$ ) $\delta 7.89-7.68(\mathrm{~m}, 3 \mathrm{H}), 7.63(\mathrm{~s}, 1 \mathrm{H}), 7.55-7.30$ $(\mathrm{m}, 3 \mathrm{H}), 7.26(\mathrm{~s}, 3 \mathrm{H}), 7.14(\mathrm{~d}, J=7.7 \mathrm{~Hz}, 3 \mathrm{H}), 2.57(\mathrm{~m}, 2 \mathrm{H}), 2.47(\mathrm{t}, J=7.9 \mathrm{~Hz}, 1 \mathrm{H}), 1.97(\mathrm{~m}$, $1 \mathrm{H}), 1.79(\mathrm{~m}, 1 \mathrm{H}), 1.63(\mathrm{~m}, 2 \mathrm{H}), 1.45-1.28(\mathrm{~m}, 2 \mathrm{H}), 1.18(\mathrm{~d}, J=8.2 \mathrm{~Hz}, 12 \mathrm{H}) .{ }^{13} \mathrm{C}$ NMR $(126$ $\mathrm{MHz}$, Chloroform- $d$ ) $\delta$ 142.9, 141.1, 133.9, 131.9, 128.5, 128.3, 127.8, 127.8, 127.6, 127.6, 126.3, 125.7, 125.6, 124.9, 83.4, 35.9, 32.4, 31.6, 29.1, 24.7, 24.7. ${ }^{11} \mathrm{~B}$ NMR (96 MHz, Chloroform- $d$ ) $\delta$ 32.8. GCMS (EI) calculated for [M]+ 400.26, found 400.3. FTIR (neat, $\left.\mathrm{cm}^{-1}\right)$ : 3059(s), 2978(s), 2930(s), 2857(s), 1632(m), 1601(s), 1506(m), 1454(s), 1370(s), 1329(s), 1269(m), 1210(m), 1135(m), 968(m), 856(s), 748(s), 699(s).<smiles>C=Cc1ccc(C(CCCCc2ccccc2)c2ccccc2)cc1</smiles>

2-[1-(4-ethenylphenyl)-5-phenylpentyl]-4,4,5,5-tetramethyl-1,3,2-dioxaborolane

(18), compound was prepared according to reported procedure using $\mathrm{IPrCuCl}$ instead of $\mathrm{IPrCuO} t-\mathrm{Bu}$ and $\mathrm{NaO} t$-Bu instead of $\mathrm{KO} t$ - $\mathrm{Bu}$, and was purified by silica gel chromatography, $0-100 \% \mathrm{DCM}$ in hexanes, and was isolated as a clear colorless liquid (188 mg, 86\% yield). ${ }^{1} \mathrm{H}$ NMR (300 MHz, Chloroform- $d$ ) $\delta 7.37-7.22(\mathrm{~m}, 4 \mathrm{H}), 7.15(\mathrm{dd}, J=15.3,8.7 \mathrm{~Hz}, 6 \mathrm{H}), 6.70(\mathrm{dd}, J=17.6,10.9 \mathrm{~Hz}$, $1 \mathrm{H}), 5.70(\mathrm{~d}, J=17.6 \mathrm{~Hz}, 1 \mathrm{H}), 5.18(\mathrm{~d}, J=10.9 \mathrm{~Hz}, 1 \mathrm{H}), 2.58(\mathrm{t}, J=7.7 \mathrm{~Hz}, 2 \mathrm{H}), 2.30(\mathrm{t}, J=7.9$ $\mathrm{Hz}, 1 \mathrm{H}), 1.87(\mathrm{dt}, J=13.2,7.9 \mathrm{~Hz}, 1 \mathrm{H}), 1.77-1.55(\mathrm{~m}, 2 \mathrm{H}), 1.43-1.27(\mathrm{~m}, 2 \mathrm{H}), 1.19(\mathrm{~d}, J=7.0$ $\mathrm{Hz}, 12 \mathrm{H}) .{ }^{13} \mathrm{C}$ NMR $(126 \mathrm{MHz}$, Chloroform- $d) \delta 142.9,137.0,134.7,128.6,128.5,128.3,126.3$, 125.6, 112.6, 111.0, 83.4, 35.9, 32.5, 31.5, 29.0, 24.7, 24.7. ${ }^{11} \mathrm{~B}$ NMR $(96 \mathrm{MHz}, \mathrm{Chloroform}-d) \delta$ 
33.4. GCMS (EI) calculated for $[\mathrm{M}]+376.26$, found 376.3. FTIR (neat, $\left.\mathrm{cm}^{-1}\right): 3059(\mathrm{~m}), 2978(\mathrm{~s})$, 2930(s), 2857(m), 1605(m), 1509(s), 1454(s), 1371(s), 1326(s), 1143(s), 851(m), 699(m).<smiles>BrC(CCCCc1ccccc1)c1ccc2occc2c1</smiles>

2-[1-(1-benzofuran-5-yl)-5-phenylpentyl]-4,4,5,5-tetramethyl-1,3,2-dioxaborolane

(19), compound was prepared according to reported procedure and was purified by silica gel column chromatography $0-10 \%$ EtOAc in hexanes and was isolated as a clear colorless liquid (180 $\mathrm{mg}$, 92\% yield). ${ }^{1} \mathrm{H}$ NMR $(300 \mathrm{MHz}$, Chloroform- $d) \delta 7.56(\mathrm{~d}, J=2.2 \mathrm{~Hz}, 1 \mathrm{H}), 7.48(\mathrm{~d}, J=8.0 \mathrm{~Hz}$, 1H), $7.39(\mathrm{~s}, 1 \mathrm{H}), 7.26(\mathrm{~s}, 2 \mathrm{H}), 7.21-7.07(\mathrm{~m}, 4 \mathrm{H}), 6.77-6.68(\mathrm{~m}, 1 \mathrm{H}), 2.68-2.50(\mathrm{~m}, 2 \mathrm{H}), 2.43$ $(\mathrm{t}, J=7.9 \mathrm{~Hz}, 1 \mathrm{H}), 2.03-1.85(\mathrm{~m}, 1 \mathrm{H}), 1.84-1.57(\mathrm{~m}, 3 \mathrm{H}), 1.49-1.30(\mathrm{~m}, 2 \mathrm{H}), 1.20(\mathrm{~d}, J=8.1$ $\mathrm{Hz}, 12 \mathrm{H}) .{ }^{13} \mathrm{C}$ NMR $(126 \mathrm{MHz}$, Chloroform-d) $\delta 155.6,144.3,142.9,140.3,128.5,128.3,125.6$, $124.8,123.8,120.7,111.0,106.5,83.4,35.9,33.0,31.6,29.0,24.8,24.7 .{ }^{11} \mathrm{~B}$ NMR (96 MHz, Chloroform- $d$ ) $\delta$ 32.1. GCMS (EI) calculated for $[\mathrm{M}]+390.24$, found 390.2. FTIR (neat, $\mathrm{cm}^{-1}$ ): 3027(m), 2977(m), 2927(s), 1621(m), 1531(m), 1454(s), 1370(m), 1323(m), 1266(m), 1142(s), 1028(m), 860(m), 733(m).

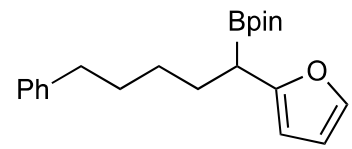

2-[1-(furan-2-yl)-5-phenylpentyl]-4,4,5,5-tetramethyl-1,3,2-dioxaborolane (20), compound was prepared according to reported procedure using IPrCuCl instead of IPrCuOt-Bu and $\mathrm{NaO} t-\mathrm{Bu}$ instead of $\mathrm{KO} t-\mathrm{Bu}$, and was purified by $\mathrm{HPLC}, 100 \%$ hexanes after, silica gel column chromatography $0-10 \%$ EtOAc in hexanes and was isolated as a clear colorless liquid. GC yield (82\% yield). ${ }^{1} \mathrm{H}$ NMR (300 MHz, Chloroform- $d$ ) $\delta 7.37(\mathrm{~s}, 1 \mathrm{H}), 7.33-7.22(\mathrm{~m}, 8 \mathrm{H}), 6.26(\mathrm{dd}, J$ $=3.2,1.9 \mathrm{~Hz}, 1 \mathrm{H}), 6.00(\mathrm{~d}, J=3.4 \mathrm{~Hz}, 1 \mathrm{H}), 2.45(\mathrm{t}, J=7.5 \mathrm{~Hz}, 1 \mathrm{H}), 2.20(\mathrm{q}, J=7.5 \mathrm{~Hz}, 2 \mathrm{H}), 1.77$ $(\mathrm{q}, J=7.8 \mathrm{~Hz}, 3 \mathrm{H}), 1.69-1.57(\mathrm{~m}, 2 \mathrm{H}), 1.36(\mathrm{q}, J=8.2 \mathrm{~Hz}, 1 \mathrm{H}), 1.22(\mathrm{~d}, J=2.7 \mathrm{~Hz}, 13 \mathrm{H}) .{ }^{13} \mathrm{C}$ NMR (126 MHz, Chloroform-d) $\delta 142.7,141.8,128.5,128.4,125.8,125.7,109.2,106.2,83.0$, 35.8, 34.9, 31.3, 29.8, 24.9, 24.7, 24.7. ${ }^{11} \mathrm{~B}$ NMR (96 MHz, Chloroform- $d$ ) $\delta 32.4$ GCMS (EI) calculated for $[\mathrm{M}]+340.27$, found 340.3. FTIR (neat, $\left.\mathrm{cm}^{-1}\right): 3024(\mathrm{~m}), 2976(\mathrm{~m}), 2927(\mathrm{~s}), 1616(\mathrm{~m})$, 1530(m), 1454(s), 1372(m), 1319(m), 1265(m), 1140(s), 1036(m), 861(m), 733(m).<smiles>c1ccc(CCCCC(c2ccccc2)c2cccs2)cc1</smiles>

4,4,5,5-tetramethyl-2-[5-phenyl-1-(thiophen-2-yl)pentyl]-1,3,2-dioxaborolane (21), compound was prepared according to reported procedure and was purified by silica gel chromatography, 0 $100 \%$ DCM in hexanes, and was isolated as a clear colorless liquid (139 mg, 78\% yield). ${ }^{1} \mathrm{H} \mathrm{NMR}$ (300 MHz, Chloroform- $d$ ) $\delta 7.25(\mathrm{~d}, J=8.1 \mathrm{~Hz}, 4 \mathrm{H}), 7.19-7.11(\mathrm{~m}, 3 \mathrm{H}), 7.07$ (dd, $J=5.2,1.1$ $\mathrm{Hz}, 1 \mathrm{H}), 6.90(\mathrm{dd}, J=5.2,3.4 \mathrm{~Hz}, 1 \mathrm{H}), 6.79(\mathrm{~d}, J=3.5 \mathrm{~Hz}, 1 \mathrm{H}), 2.61(\mathrm{~m}, 3 \mathrm{H}), 1.97-1.55(\mathrm{~m}$, 4H), 1.39 (m, 2H), 1.21 (d, $J=4.8 \mathrm{~Hz}, 12 \mathrm{H}) .{ }^{13} \mathrm{C}$ NMR (126 MHz, Chloroform-d) $\delta 146.6,142.8$, $128.5,128.3,126.8,125.7,123.8,122.5,83.7,35.9,33.8,31.4,28.8,24.9,24.8,24.7 .{ }^{11} \mathrm{~B}$ NMR $(96 \mathrm{MHz}$, Chloroform- $d$ ) $\delta 32.1$. GCMS (EI) calculated for [M]+356.26, found 356.3. FTIR (neat, 
$\left.\mathrm{cm}^{-1}\right):$ 3064(m), 3064(m), 3026(m), 2978(s), 2931(s), 2857(s), 1604(s), 1496(s), 1454(m), 1370(s), 1327(s), 1143(s), 1030(m), 966(m), 849(m), 750(m), 697(m).<smiles>c1ccc(CCCCC(c2ccccc2)c2cnc3ccccc3c2)cc1</smiles>

3-[5-phenyl-1-(4,4,5,5-tetramethyl-1,3,2-dioxaborolan-2-yl)pentyl]quinoline (22), compound was prepared according to reported procedure and was purified by prep TLC, $2 \%$ Triethylamine, $20 \%$ EtOAc in hexanes and was isolated as a clear colorless liquid (148 mg, 74\% yield). ${ }^{1} \mathrm{H} \mathrm{NMR}$ $(500 \mathrm{MHz}$, Chloroform- $d$ ) $\delta 8.88(\mathrm{dd}, J=4.3,1.8 \mathrm{~Hz}, 1 \mathrm{H}), 8.06(\mathrm{dd}, J=17.9,7.6 \mathrm{~Hz}, 2 \mathrm{H}), 7.67-$ $7.59(\mathrm{~m}, 2 \mathrm{H}), 7.38(\mathrm{dd}, J=8.3,4.2 \mathrm{~Hz}, 1 \mathrm{H}), 7.28-7.19(\mathrm{~m}, 2 \mathrm{H}), 7.19-7.12(\mathrm{~m}, 3 \mathrm{H}), 2.58(\mathrm{~m}$, 2H), $2.49(\mathrm{t}, J=7.8 \mathrm{~Hz}, 1 \mathrm{H}), 1.99(\mathrm{~m}, 1 \mathrm{H}), 1.80(\mathrm{~m}, 1 \mathrm{H}), 1.65(\mathrm{~m}, 2 \mathrm{H}), 1.39-1.35(\mathrm{~m}, 2 \mathrm{H}), 1.19$ $(\mathrm{d}, J=7.9 \mathrm{~Hz}, 12 \mathrm{H}) .{ }^{13} \mathrm{C}$ NMR $(126 \mathrm{MHz}$, Chloroform- $d$ ) $\delta 151.1,146.1,136.9,129.5,129.3$, $128.8,127.3,126.7,117.0,83.6,35.9,32.9,31.5,29.0,25.0,24.7,24.7 .{ }^{11} \mathrm{~B}$ NMR (96 MHz, Chloroform- $d$ ) $\delta$ 33.1. GCMS (EI) calculated for $[\mathrm{M}]+401.25$, found 400.3. FTIR (neat, $\mathrm{cm}^{-1}$ ): 3095 (m), 3062(s), 2978(s), 2924(s), 2860(s), 1630(m), 1604(s), 1509(m), 1450(s), 1372(s), 1322(s), 1269(m), 1210(m), 1134(m), 968(m), 856(s), 748(s), 699(s).<smiles>c1ccc(CCCCC(c2ccccc2)c2cnc(N3CCOCC3)nc2)cc1</smiles>

\section{4-\{5-[5-phenyl-1-(4,4,5,5-tetramethyl-1,3,2-dioxaborolan-2-yl)pentyl]pyrimidin-2-}

yl\}morpholine (23), compound was prepared according to reported procedure using $\mathrm{IPrCuCl}$ instead of IPrCuOt-Bu, KOTMS instead of $\mathrm{KO} t$-Bu and a 1:1 mixture of toluene and THF. Compound was purified by prep TLC, $2 \%$ Triethylamine, 20\% EtOAc in hexanes and was isolated as a clear colorless liquid (178 mg, 81\% yield). ${ }^{1} \mathrm{H}$ NMR $(300 \mathrm{MHz}$, Chloroform- $d$ ) $\delta 8.37(\mathrm{~s}, 2 \mathrm{H})$, $7.43-7.13(\mathrm{~m}, 7 \mathrm{H}), 3.84(\mathrm{qq}, J=4.8,2.3 \mathrm{~Hz}, 10 \mathrm{H}), 2.30(\mathrm{t}, J=7.9 \mathrm{~Hz}, 1 \mathrm{H}), 2.00-1.79(\mathrm{~m}, 1 \mathrm{H})$, $1.74-1.46(\mathrm{~m}, 3 \mathrm{H}), 1.46-1.20(\mathrm{~m}, 2 \mathrm{H}), 1.16(\mathrm{~d}, J=6.7 \mathrm{~Hz}, 12 \mathrm{H}) .{ }^{13} \mathrm{C} \mathrm{NMR}(126 \mathrm{MHz}$, Chloroform- $d$ ) $\delta 155.2,129.3,128.6,128.4,125.9,123.9,120.8,83.7,66.9,44.6,35.5,32.8,31.1$, 29.8, 25.1, 24.8, 24.8. ${ }^{11} \mathrm{~B}$ NMR (96 MHz, Chloroform- $d$ ) $\delta$ 32.7. GCMS (EI) calculated for [M]+ 437.28, found 437.4. FTIR (neat, $\left.\mathrm{cm}^{-1}\right): 3104(\mathrm{~m}), 3095(\mathrm{~m}), 3062(\mathrm{~s}), 2978(\mathrm{~s}), 2924(\mathrm{~s}), 2860(\mathrm{~s})$, 1630(m), 1604(s), 1509(m), 1449(s), 1401(s), 1375(s), 1319(s), 1299(m), 1269(m), 1209(m), 1132(m), 1101(m), 966(m), 856(s), 748(s), 699(s).

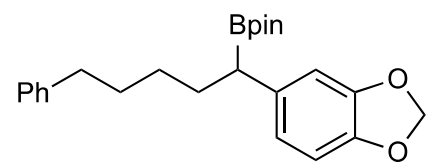

2-[1-(2H-1,3-benzodioxol-5-yl)-5-phenylpentyl]-4,4,5,5-tetramethyl-1,3,2-dioxaborolane (24), compound was prepared according to reported procedure and was purified by silica gel column chromatography, 0-10\% EtOAc in hexanes and was isolated as a clear colorless liquid (184 mg, 93\% yield). ${ }^{1} \mathrm{H}$ NMR (300 MHz, Chloroform-d) $\delta 7.33-7.07(\mathrm{~m}, 7 \mathrm{H}), 6.77-6.56(\mathrm{~m}, 3 \mathrm{H}), 5.90$ $(\mathrm{s}, 2 \mathrm{H}), 2.63-2.48(\mathrm{~m}, 2 \mathrm{H}), 2.21(\mathrm{t}, J=7.9 \mathrm{~Hz}, 1 \mathrm{H}), 1.92-1.71(\mathrm{~m}, 1 \mathrm{H}), 1.70-1.52(\mathrm{~m}, 3 \mathrm{H})$, $1.30(\mathrm{~d}, \mathrm{~J}=16.1 \mathrm{~Hz}, 4 \mathrm{H}), 1.18(\mathrm{~d}, J=6.8 \mathrm{~Hz}, 12 \mathrm{H}) .{ }^{13} \mathrm{C}$ NMR $(126 \mathrm{MHz}$, Chloroform- $d$ ) $\delta 147.5$, 
145.1, 142.8, 137.2, 128.4, 128.2, 125.6, 121.2, 108.9, 108.2, 100.6, 83.4, 35.9, 32.8, 31.5, 28.8, 25.5, 24.7, 24.6. ${ }^{11} \mathrm{~B}$ NMR (96 MHz, Chloroform- $d$ ) $\delta$ 33.0. GCMS (EI) calculated for [M]+ 394.32, found 394.3. FTIR (neat, $\left.\mathrm{cm}^{-1}\right)$ : 3062(w), 3021(m), 2984(m), 2934(m), 1672(w), 1604(m), 1489(s), 1444(s), 1246(s), 1142(s), 1040(m), 933(m), 851(m), 755(m).<smiles>COc1ccc(C(CCCCC#N)c2ccccc2)cc1</smiles>

6-(4-methoxyphenyl)-6-(4,4,5,5-tetramethyl-1,3,2-dioxaborolan-2-yl)hexanenitrile

(25), compound was prepared according to reported procedure and was purified by HPLC, $0-1 \%$ IPA in hexanes, after silica gel column chromatography 0-10\% EtOAc in hexanes and was isolated as a clear colorless liquid. GC yield (76\% yield). ${ }^{1} \mathrm{H}$ NMR $(300 \mathrm{MHz}$, Chloroform- $d) \delta 7.31-7.19(\mathrm{~m}$, $3 \mathrm{H}), 6.80(\mathrm{~d}, J=8.5 \mathrm{~Hz}, 2 \mathrm{H}), 3.78(\mathrm{~s}, 3 \mathrm{H}), 2.68-2.43(\mathrm{~m}, 2 \mathrm{H}), 2.23(\mathrm{t}, J=7.9 \mathrm{~Hz}, 1 \mathrm{H}), 1.94-$ $1.71(\mathrm{~m}, 1 \mathrm{H}), 1.71-1.58(\mathrm{~m}, 3 \mathrm{H}), 1.38-1.24(\mathrm{~m}, 2 \mathrm{H}), 1.18(\mathrm{~d}, J=7.3 \mathrm{~Hz}, 12 \mathrm{H}) .{ }^{13} \mathrm{C}$ NMR $(126$ MHz, Chloroform- $d$ ) $\delta 131.6,127.4,125.5,119.8,114.1,83.5,58.9,31.8,29.8,29.5,25.3,24.7$, 16.5. ${ }^{11} \mathrm{~B}$ NMR (96 MHz, Chloroform- $d$ ) $\delta$ 33.2. GCMS (EI) calculated for $[\mathrm{M}]+375.24$, found 375.2. FTIR (neat, $\left.\mathrm{cm}^{-1}\right)$ : 3057(m), 2926(s), 2849(s), 2247(s), 2606(s), 1515(s), 1371(s), 1243(s), 1143(s), 1032(s), 967(s), 833(m), 751(m).<smiles>COc1ccc(C(CCCCc2ccccc2)c2ccccc2)cc1</smiles>

2-[1-(4-methoxyphenyl)-5-phenoxypentyl]-4,4,5,5-tetramethyl-1,3,2-dioxaborolane

(26), compound was prepared according to reported procedure and was purified by silica gel chromatography, $0-100 \%$ DCM in hexanes, and was isolated as a clear colorless liquid (185 mg, 93\% yield). ${ }^{1} \mathrm{H}$ NMR $(300 \mathrm{MHz}$, Chloroform- $d) \delta 7.31(\mathrm{~s}, 3 \mathrm{H}), 7.11(\mathrm{~d}, J=8.6 \mathrm{~Hz}, 2 \mathrm{H}), 6.80(\mathrm{~d}$, $J=8.6 \mathrm{~Hz}, 2 \mathrm{H}), 4.47(\mathrm{~s}, 2 \mathrm{H}), 3.77(\mathrm{~s}, 3 \mathrm{H}), 3.43(\mathrm{t}, J=6.7 \mathrm{~Hz}, 2 \mathrm{H}), 2.23(\mathrm{t}, J=7.9 \mathrm{~Hz}, 1 \mathrm{H}), 1.91$ $-1.73(\mathrm{~m}, 1 \mathrm{H}), 1.71-1.51(\mathrm{~m}, 2 \mathrm{H}), 1.43-1.23(\mathrm{~m}, 2 \mathrm{H}), 1.19(\mathrm{~d}, J=6.0 \mathrm{~Hz}, 13 \mathrm{H}) .{ }^{13} \mathrm{C} \mathrm{NMR}$ (126 MHz, Chloroform-d) $\delta 157.4,138.9,135.4,129.3,128.4,127.6,127.4,113.8,83.3,72.8$, 70.5, 55.2, 32.7, 29.8, 25.8, 24.7, 24.7. ${ }^{11} \mathrm{~B}$ NMR (96 MHz, Chloroform- $d$ ) $\delta$ 31.9. GCMS (EI) calculated for $[\mathrm{M}]+396.25$, found 396.3. FTIR (neat, $\left.\mathrm{cm}^{-1}\right): 3051(\mathrm{~m}), 2978(\mathrm{~s}), 2932(\mathrm{~s}), 2858(\mathrm{~m})$, 1601(m), 1510(s), 1454(m), 1370(m), 1325(m), 1247(m), 1143(s), 1031(m), 851(m), 755(s).

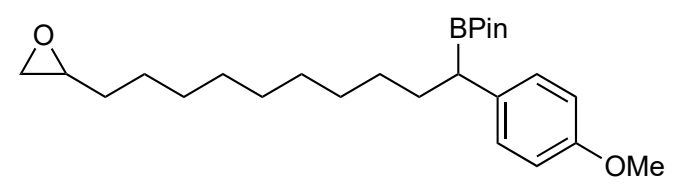

2-[1-(4-methoxyphenyl)-10-(oxiran-2-yl)decyl]-4,4,5,5-tetramethyl-1,3,2-dioxaborolane (27), compound was prepared according to reported procedure and was purified by silica gel column chromatography, 0-10\% EtOAc in hexanes and was isolated as a clear colorless liquid (193 mg, 93\% yield). ${ }^{1} \mathrm{H}$ NMR (300 MHz, Chloroform- $d$ ) $\delta 7.11(\mathrm{~d}, J=8.6 \mathrm{~Hz}, 2 \mathrm{H}), 6.80(\mathrm{~d}, J=8.6 \mathrm{~Hz}$, $2 \mathrm{H}), 3.77(\mathrm{~s}, 3 \mathrm{H}), 2.94-2.84(\mathrm{~m}, 1 \mathrm{H}), 2.79-2.69(\mathrm{~m}, 1 \mathrm{H}), 2.46(\mathrm{dd}, J=5.1,2.7 \mathrm{~Hz}, 1 \mathrm{H}), 2.22(\mathrm{t}$, $J=7.9 \mathrm{~Hz}, 1 \mathrm{H}), 1.89-1.69(\mathrm{~m}, 1 \mathrm{H}), 1.67-1.36(\mathrm{~m}, 6 \mathrm{H}), 1.37-1.13(\mathrm{~m}, 26 \mathrm{H}) .{ }^{13} \mathrm{C}$ NMR $(126$ MHz, Chloroform- $d$ ) $\delta$ 157.3, 135.5, 129.3, 113.8, 83.3, 55.2, 52.5, 47.2, 33.0, 32.6, 29.7, 29.6, 29.6, 29.5, 29.3, 26.1, 24.9, 24.7, 24.7. ${ }^{11} \mathrm{~B}$ NMR (96 MHz, Chloroform- $d$ ) $\delta$ 32.9. GCMS (EI) 
calculated for $[\mathrm{M}]+416.31$, found 416.3. FTIR (neat, $\left.\mathrm{cm}^{-1}\right): 3045(\mathrm{~m}), 2976(\mathrm{~s}), 2926(\mathrm{~s}), 2855(\mathrm{~m})$, 1611(m), 1512(s), 1454(m), 1370(s), 1323(s), 1246(m), 1142(s), 1112(m), 1037(m), 967(m), 851(m), 754(m).<smiles>COc1ccc(C(CCCCOC2CCCCO2)c2ccccc2)cc1</smiles>

2-[1-(4-methoxyphenyl)-5-(oxan-2-yloxy)pentyl]-4,4,5,5-tetramethyl-1,3,2-dioxaborolane (28), compound was prepared according to reported procedure and was purified by prep TLC, $2 \%$ Triethylamine in DCM, and was isolated as a clear colorless liquid (176 mg, 78\% yield). ${ }^{1} \mathrm{H}$ NMR $(300 \mathrm{MHz}$, Chloroform- $d$ ) $\delta$ 7.95, (d, $J=8.8 \mathrm{~Hz}, 2 \mathrm{H}), 6.93(\mathrm{~d}, \mathrm{~J}=8.9 \mathrm{~Hz}, 2 \mathrm{H}), 4.59(\mathrm{t}, J=3.5 \mathrm{~Hz}$, $1 \mathrm{H}), 3.94-3.71(\mathrm{~m}, 5 \mathrm{H}), 2.96(\mathrm{t}, J=7.3 \mathrm{~Hz}, 2 \mathrm{H}), 1.90-1.77(\mathrm{~m}, 3 \mathrm{H}), 1.78-1.45(\mathrm{~m}, 10 \mathrm{H}), 1.25$ (s, 12H). ${ }^{13} \mathrm{C}$ NMR (126 MHz, Chloroform-d) $\delta 145.2,136.4,130.5,113.8,99.0,83.5,67.4,62.5$, 55.6, 38.1 , 30.9, 29.9, 25.7, 24.9, 24.8, 24.7, 22.8, 21.6, 19.8. ${ }^{11} \mathrm{~B}$ NMR (96 MHz, Chloroform- $d$ ) $\delta$ 32.0. GCMS (EI) calculated for [M]+ 404.27, found 404.3. FTIR (neat, $\left.\mathrm{cm}^{-1}\right): 3035(\mathrm{~m}), 2972(\mathrm{~s})$, 2929(s), 2849(m), 1609(s), 1510(s), 1455(m), 1373(s), 1323(s), 1241(m), 1139(s), 1111(m), 1040(m), 966(m), 851(m), 755(m), 699(m).<smiles>FC(F)(F)c1ccc(C(CCCCCCl)c2ccccc2)cc1</smiles>

\section{2-\{6-chloro-1-[4-(trifluoromethyl)phenyl]hexyl\}-4,4,5,5-tetramethyl-1,3,2-dioxaborolane}

(29), compound was prepared according to reported procedure using $\mathrm{IPrCuCl}$ instead of $\mathrm{IPrCuO} t$ $\mathrm{Bu}$ and $\mathrm{NaO} t$ - $\mathrm{Bu}$ instead of $\mathrm{KO} t$-Bu, and was purified by silica gel chromatography, $0-100 \% \mathrm{DCM}$ in hexanes, and was isolated as a clear colorless liquid (163 mg, 83\% yield). ${ }^{1} \mathrm{H}$ NMR (300 MHz, Chloroform- $d$ ) $\delta 7.50(\mathrm{~d}, J=8.1 \mathrm{~Hz}, 2 \mathrm{H}), 7.30(\mathrm{~d}, J=8.0 \mathrm{~Hz}, 2 \mathrm{H}), 3.50(\mathrm{t}, J=6.7 \mathrm{~Hz}, 2 \mathrm{H}), 2.36$ $(\mathrm{t}, J=7.9 \mathrm{~Hz}, 1 \mathrm{H}), 1.99-1.58(\mathrm{~m}, 5 \mathrm{H}), 1.46-1.38(\mathrm{~m}, 2 \mathrm{H}), 1.30-1.25(\mathrm{~m}, 4 \mathrm{H}), 1.20(\mathrm{~d}, J=4.4$ $\mathrm{Hz}, 11 \mathrm{H}) .{ }^{13} \mathrm{C}$ NMR $(126 \mathrm{MHz}$, Chloroform- $d$ ) $\delta 147.6,128.4,127.6$ (q, $J=34.7 \mathrm{~Hz}), 125.1$ (q, $J$ $=5.5 \mathrm{~Hz}), 124.7$ (q, $J=265.3 \mathrm{~Hz}), 83.4,33.7,32.6,32.0,28.5,27.9,24.6,24.5,24.5 .{ }^{19} \mathrm{~F} \mathrm{NMR}$ (470 MHz, Chloroform- $d$ ) $\delta-65.0 .{ }^{11} \mathrm{~B}$ NMR (96 MHz, Chloroform- $d$ ) $\delta$ 32.3. GCMS (EI) calculated for $[\mathrm{M}]+390.17$, found 390.2. FTIR (neat, $\left.\mathrm{cm}^{-1}\right): 3029(\mathrm{~m}), 2985(\mathrm{~s}), 2931(\mathrm{~s}), 2861(\mathrm{~s})$, 1617(s), 1368(m), 1325(s), 1318(m), 1172(m), 1120(s), 1054(m), 1018(s), 967(m), 852(m), 699(m).<smiles>FC(F)(F)c1ccc(C(CCCCCCBr)c2ccccc2)cc1</smiles>

2-\{7-bromo-1-[4-(trifluoromethyl)phenyl]heptyl\}-4,4,5,5-tetramethyl-1,3,2-dioxaborolane (30), compound was prepared according to reported procedure using $\mathrm{IPrCuCl}$ instead of $\mathrm{IPrCuO} t$ $\mathrm{Bu}$ and $\mathrm{NaO} t$ - $\mathrm{Bu}$ instead of $\mathrm{KO} t$ - $\mathrm{Bu}$, and was purified by silica gel chromatography, $0-100 \% \mathrm{DCM}$ in hexanes, and was isolated as a clear colorless liquid (182 mg, 81\% yield). ${ }^{1} \mathrm{H}$ NMR (300 MHz, Chloroform- $d$ ) $\delta 7.49(\mathrm{~d}, J=8.1 \mathrm{~Hz}, 2 \mathrm{H}), 7.30(\mathrm{~d}, J=8.0 \mathrm{~Hz}, 2 \mathrm{H}), 3.38(\mathrm{t}, J=6.9 \mathrm{~Hz}, 2 \mathrm{H}), 2.36$ $(\mathrm{t}, J=7.9 \mathrm{~Hz}, 1 \mathrm{H}), 1.91-1.73(\mathrm{~m}, 3 \mathrm{H}), 1.74-1.54(\mathrm{~m}, 1 \mathrm{H}), 1.49-1.35(\mathrm{~m}, 2 \mathrm{H}), 1.35-1.20(\mathrm{~m}$, $6 \mathrm{H}), 1.19(\mathrm{~d}, J=4.3 \mathrm{~Hz}, 12 \mathrm{H}) .{ }^{13} \mathrm{C}$ NMR $(126 \mathrm{MHz}$, Chloroform- $d$ ) $\delta 147.9,127.9$ (q, $J=34.7$ 
Hz), 125.3 (q, $J=5.5 \mathrm{~Hz}), 124.9$ (q, $J=265.3 \mathrm{~Hz}), 83.7,34.0,32.9,32.3,29.1,28.8,28.1$, 24.9, 24.7, 24.7. ${ }^{19} \mathrm{~F}$ NMR (470 MHz, Chloroform- $d$ ) $\delta-65.1 .{ }^{11} \mathrm{~B}$ NMR $(96 \mathrm{MHz}$, Chloroform- $d$ ) $\delta 31.6$. GCMS (EI) calculated for [M]+448.14, found 448.2. FTIR (neat, $\left.\mathrm{cm}^{-1}\right): 2984(\mathrm{~m}), 2931(\mathrm{~s}), 2858(\mathrm{~s})$, 1617(s), 1465(m), 1371(s), 1164(m), 1123(s), 1018(s), 967(m), 852(m).<smiles>CC(C)(C)OCCCCC(c1ccccc1)c1ccc(C(F)(F)F)cc1</smiles>

tert-butyldimethyl\{[5-(4,4,5,5-tetramethyl-1,3,2-dioxaborolan-2-yl)-5-[4(trifluoromethyl)phenyl]pentyl]oxy\}silane (31), compound was prepared according to reported procedure using IPrCuCl instead of IPrCuOt-Bu and $\mathrm{NaO} t$-Bu instead of $\mathrm{KO} t-\mathrm{Bu}$, and was purified by silica gel chromatography $0-100 \%$ DCM in hexanes, and was isolated as a clear colorless liquid (185 mg, 82\% yield). ${ }^{1} \mathrm{H}$ NMR (300 MHz, Chloroform- $d$ ) $\delta 7.49(\mathrm{~d}, J=8.1 \mathrm{~Hz}, 2 \mathrm{H}), 7.30(\mathrm{~d}, J=$ $8.1 \mathrm{~Hz}, 2 \mathrm{H}), 3.56(\mathrm{t}, J=6.5 \mathrm{~Hz}, 2 \mathrm{H}), 2.36(\mathrm{t}, J=7.9 \mathrm{~Hz}, 1 \mathrm{H}), 1.98-1.77(\mathrm{~m}, 1 \mathrm{H}), 1.74-1.59(\mathrm{~m}$, $1 \mathrm{H}), 1.59-1.42(\mathrm{~m}, 2 \mathrm{H}), 1.41-1.13(\mathrm{~m}, 19 \mathrm{H}), 0.88(\mathrm{~s}, 9 \mathrm{H}), 0.02(\mathrm{~s}, 6 \mathrm{H}) .{ }^{13} \mathrm{C} \mathrm{NMR}(126 \mathrm{MHz}$, Chloroform- $d$ ) $\delta$ 148.0, 128.7, $127.3(\mathrm{q}, J=31.1 \mathrm{~Hz}), 125.3(\mathrm{q}, J=2.5 \mathrm{~Hz}), 124.7(\mathrm{q}, J=279.3$ $\mathrm{Hz}), 83.6,63.3,32.8,32.4,26.1,25.9,24.9,24.9,24.7,24.7,18.5,-5.1 .{ }^{19} \mathrm{~F}$ NMR (470 MHz, Chloroform- $d$ ) $\delta-62.1 .{ }^{11} \mathrm{~B}$ NMR $(96 \mathrm{MHz}$, Chloroform- $d$ ) $\delta 33.4$. GCMS (EI) calculated for [M]+ 486.29, found 486.3. FTIR (neat, $\left.\mathrm{cm}^{-1}\right): 3057(\mathrm{~m}), 2930(\mathrm{~s}), 2858(\mathrm{~s}), 1617(\mathrm{~s}), 1473(\mathrm{~m}), 1372(\mathrm{~m})$, 1325(s), 1257(m), 1164(s), 1124(s), 1068(s), 835(m), 699(m).

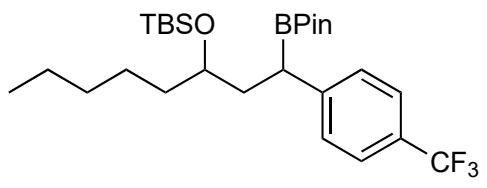

tert-butyl(\{[1-(4-methoxyphenyl)-1-(4,4,5,5-tetramethyl-1,3,2-dioxaborolan-2-yl)octan-3yl]oxy\})dimethylsilane (32), compound was prepared according to reported procedure using $\mathrm{IPrCuCl}$ instead of $\mathrm{IPrCuO} t-\mathrm{Bu}$ and $\mathrm{NaO} t-\mathrm{Bu}$ instead of $\mathrm{KO} t-\mathrm{Bu}$, and was purified by prep TLC, $2 \%$ Triethylamine in DCM and was isolated as a clear colorless liquid (199 mg, 76\% yield). ${ }^{1} \mathrm{H}$ NMR (300 MHz, Chloroform-d) $\delta 7.49(\mathrm{~d}, \mathrm{~J}=8.0 \mathrm{~Hz}, 2 \mathrm{H}), 7.30(\mathrm{~d}, J=8.2 \mathrm{~Hz}, 2 \mathrm{H}), 3.57$ (dt, $J=$ $12.1,5.9 \mathrm{~Hz}, 1 \mathrm{H}), 2.56(\mathrm{t}, J=7.7 \mathrm{~Hz}, 1 \mathrm{H}), 1.93-1.64(\mathrm{~m}, 2 \mathrm{H}), 1.32-1.26(\mathrm{~m}, 11 \mathrm{H}), 1.17(\mathrm{~d}, J=$ $3.2 \mathrm{~Hz}, 12 \mathrm{H}), 0.88(\mathrm{~s}, 9 \mathrm{H}),-0.01(\mathrm{~s}, 6 \mathrm{H}) .{ }^{13} \mathrm{C} \mathrm{NMR}(126 \mathrm{MHz}$, Chloroform-d) $\delta 132.2,129.8$ (q, $J=32.7 \mathrm{~Hz}), 127.0(\mathrm{q}, J=3.4 \mathrm{~Hz}), 126.6,125.1(\mathrm{q}, J=272.2 \mathrm{~Hz}), 85.9,83.4,38.7,31.6,25.9$, 25.0, 24.3, 24.0, 23.9, 22.7, 18.4, 14.2, -4.4, -5.0. ${ }^{19} \mathrm{~F}$ NMR (470 MHz, Chloroform-d) -62.3. ${ }^{11} \mathrm{~B}$ NMR (96 MHz, Chloroform- $d$ ) $\delta$ 33.3. GCMS (EI) calculated for [M]+ 514.33, found 499.3. FTIR (neat, $\left.\mathrm{cm}^{-1}\right)$ : 3058(m), 2929(s), 2860(s), 1617(s), 1474(m), 1376(m), 1326(s), 1254(m), 1160(s), 1122(s), $1069(\mathrm{~s}), 835(\mathrm{~m}), 699(\mathrm{~m})$.

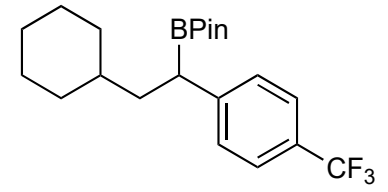

2-\{2-cyclohexyl-1-[4-(trifluoromethyl)phenyl]ethyl\}-4,4,5,5-tetramethyl-1,3,2-dioxaborolane (33), compound was prepared according to reported procedure using $\mathrm{IPrCuCl}$ instead of $\mathrm{IPrCuO} t$ $\mathrm{Bu}$ and $\mathrm{NaO} t$ - $\mathrm{Bu}$ instead of $\mathrm{KO} t$ - $\mathrm{Bu}$, and was purified by silica gel chromatography $0-100 \% \mathrm{DCM}$ in hexanes, and was isolated as a clear colorless liquid (133 mg, 77\% yield). ${ }^{1} \mathrm{H}$ NMR (300 MHz, 
Chloroform- $d$ ) $\delta 7.49$ (d, $J=8.1 \mathrm{~Hz}, 2 \mathrm{H}), 7.30(\mathrm{~d}, J=8.0 \mathrm{~Hz}, 2 \mathrm{H}), 2.52(\mathrm{t}, J=8.1 \mathrm{~Hz}, 1 \mathrm{H}), 1.86$ $-1.57(\mathrm{~m}, 7 \mathrm{H}), 1.18(\mathrm{~d}, J=2.8 \mathrm{~Hz}, 16 \mathrm{H}), 0.99-0.76(\mathrm{~m}, 3 \mathrm{H}) .{ }^{13} \mathrm{C}$ NMR $(126 \mathrm{MHz}$, Chloroformd) $\delta 148.2,128.7,127.5(\mathrm{q}, J=32.7 \mathrm{~Hz}), 125.3(\mathrm{q}, J=3.8 \mathrm{~Hz}), 124.6(\mathrm{q}, J=277.2, \mathrm{~Hz}), 83.6$, 39.9, 36.7, 33.8, 33.0, 26.8, 26.4, 26.4, 24.7, 24.7. ${ }^{19} \mathrm{~F}$ NMR (470 MHz, Chloroform- $d$ ) $\delta$-64.2. ${ }^{11} \mathrm{~B}$ NMR (96 MHz, Chloroform- $d$ ) $\delta$ 32.5. GCMS (EI) calculated for [M]+ 344.25, found 344.2. FTIR (neat, $\left.\mathrm{cm}^{-1}\right): 3051(\mathrm{~m}), 2980(\mathrm{~m}), 2924(\mathrm{~s}), 1617(\mathrm{~s}), 1448(\mathrm{~m}), 1381(\mathrm{~m}), 1324(\mathrm{~s}), 1163(\mathrm{~m})$, 1123(s), 1068(s), 1018(m), 968(m), 853(m).

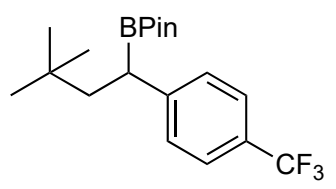

2-[1-(4-methoxyphenyl)-3,3-dimethylbutyl]-4,4,5,5-tetramethyl-1,3,2-dioxaborolane (34), compound was prepared according to reported procedure using $\mathrm{IPrCuCl}$ instead of $\mathrm{IPrCuOt}-\mathrm{Bu}$ and $\mathrm{NaOt}$ - $\mathrm{Bu}$ instead of $\mathrm{KO} t$ - $\mathrm{Bu}$, and was purified by prep TLC, $2 \%$ Triethylamine in DCM and was isolated as a clear colorless liquid (134 mg, 75\% yield). ${ }^{1} \mathrm{H}$ NMR (500 MHz, Chloroform- $d$ ) $\delta 7.48(\mathrm{~d}, J=8.1 \mathrm{~Hz}, 2 \mathrm{H}), 7.33(\mathrm{~d}, J=8.1 \mathrm{~Hz}, 2 \mathrm{H}), 2.46$ (dd, $J=9.5,4.1 \mathrm{~Hz}, 1 \mathrm{H}), 2.03$ (dd, $J=$ 13.4, $9.6 \mathrm{~Hz}, 1 \mathrm{H}), 1.49$ (dd, $J=13.3,4.0 \mathrm{~Hz}, 1 \mathrm{H}), 1.14(\mathrm{~s}, 12 \mathrm{H}), 0.90(\mathrm{~s}, 9 \mathrm{H}) .{ }^{13} \mathrm{C}$ NMR $(126 \mathrm{MHz}$, Chloroform- $d$ ) $\delta 128.5,127.7(\mathrm{q}, J=266.7 \mathrm{~Hz}), 127.5(\mathrm{q}, J=34.0 \mathrm{~Hz}), 125.3(\mathrm{q}, J=3.4 \mathrm{~Hz})$, 120.4, 83.6, 46.5, 31.6, 29.8, 24.7, 24.6, 24.2. ${ }^{19} \mathrm{~F}$ NMR (470 MHz, Chloroform- $d$ ) $\delta-64.1 .{ }^{11} \mathrm{~B}$ NMR (96 MHz, Chloroform- $d$ ) $\delta$ 32.4. GCMS (EI) calculated for $[\mathrm{M}]+356.21$, found 356.2. FTIR (neat, $\left.\mathrm{cm}^{-1}\right): 3052(\mathrm{~m}), 2979(\mathrm{~m}), 2924(\mathrm{~s}), 1615(\mathrm{~s}), 1450(\mathrm{~m}), 1382(\mathrm{~m}), 1322(\mathrm{~s}), 1159(\mathrm{~m}), 1124(\mathrm{~s})$, 1070(s), 967(m), 853(m).<smiles>COc1ccc(C(CCCC2OCCO2)c2ccccc2)cc1</smiles>

2-[4-(1,3-dioxolan-2-yl)-1-(4-methoxyphenyl)butyl]-4,4,5,5-tetramethyl-1,3,2-dioxaborolane (35), compound was prepared according to reported procedure and was purified by silica gel column chromatography, $0-10 \%$ EtOAc in hexanes and was isolated as a clear colorless liquid (158 mg, 87\% yield). ${ }^{1} \mathrm{H}$ NMR (300 MHz, Chloroform- $d$ ) $\delta 7.10$ (d, $\left.J=8.6 \mathrm{~Hz}, 2 \mathrm{H}\right), 6.79$ (d, $J=$ $8.7 \mathrm{~Hz}, 2 \mathrm{H}), 4.79(\mathrm{t}, J=4.9 \mathrm{~Hz}, 1 \mathrm{H}), 3.92-3.79(\mathrm{~m}, 4 \mathrm{H}), 3.76(\mathrm{~s}, 3 \mathrm{H}), 2.23(\mathrm{t}, J=7.9 \mathrm{~Hz}, 1 \mathrm{H})$, $1.83(\mathrm{~m}, 1 \mathrm{H}), 1.70-1.59(\mathrm{~m}, 3 \mathrm{H}), 1.39(\mathrm{q}, J=7.7 \mathrm{~Hz}, 2 \mathrm{H}), 1.19(\mathrm{~d}, J=5.9 \mathrm{~Hz}, 12 \mathrm{H}) .{ }^{13} \mathrm{C}$ NMR (126 MHz, Chloroform- $d$ ) $\delta$ 157.4, 135.2, 129.3, 113.8, 104.7, 83.3, 64.9, 55.3, 34.1, 32.9, 24.8, 24.7, 23.9. ${ }^{11} \mathrm{~B}$ NMR (96 MHz, Chloroform- $d$ ) $\delta$ 32.6. GCMS (EI) calculated for $[\mathrm{M}]+362.23$, found 361.2. FTIR (neat, $\left.\mathrm{cm}^{-1}\right): 3050(\mathrm{~m}), 2974(\mathrm{~s}), 2930(\mathrm{~s}), 2851(\mathrm{~s}), 1617(\mathrm{~s}), 1452(\mathrm{~m}), 1369(\mathrm{~m})$, 1326(s), 1218(m), 1202(m), 1142(m), 1019(m), 911(w), 851(m), 699(m).<smiles>COc1ccc(C(CCCCOC(=O)C(C)(C)C)c2ccccc2)cc1</smiles>

5-(4-methoxyphenyl)-5-(4,4,5,5-tetramethyl-1,3,2-dioxaborolan-2-yl)pentyl-2,2-

dimethylpropanoate (36), compound was prepared according to reported procedure and was purified by silica gel column chromatography $0-10 \%$ EtOAc in hexanes and was isolated as a clear colorless liquid (159 mg, 79\% yield). ${ }^{1} \mathrm{H}$ NMR (300 MHz, Chloroform- $d$ ) $\delta 7.10$ (d, $J=8.6 \mathrm{~Hz}$, 
2H), $6.80(\mathrm{~d}, J=8.7 \mathrm{~Hz}, 2 \mathrm{H}), 4.00$ (t, $J=6.7 \mathrm{~Hz}, 2 \mathrm{H}), 3.77(\mathrm{~s}, 3 \mathrm{H}), 2.22$ (t, $J=7.9 \mathrm{~Hz}, 1 \mathrm{H}), 1.81$ $(\mathrm{m}, 1 \mathrm{H}), 1.62(\mathrm{~m}, 3 \mathrm{H}), 1.45-1.00(\mathrm{~m}, 27 \mathrm{H}) .{ }^{13} \mathrm{C}$ NMR $(126 \mathrm{MHz}$, Chloroform- $d) \delta$ 178.5, 157.4, 135.0, 129.2, 113.7, 83.2, 64.3, 55.1, 38.7, 32.4, 28.6, 27.2, 25.5, 24.8, 24.6, 24.6. ${ }^{11}$ B NMR $(96$ $\mathrm{MHz}$, Chloroform- $d$ ) $\delta 32.5$. GCMS (EI) calculated for $[\mathrm{M}]+404.27$, found 404.3. FTIR (neat, $\left.\mathrm{cm}^{-1}\right): 3059(\mathrm{~m}), 2976(\mathrm{~s}), 2946(\mathrm{~s}), 2834(\mathrm{~m}), 1725(\mathrm{~s}), 1609(\mathrm{~s}), 1511(\mathrm{~m}), 1462(\mathrm{~m}), 1369(\mathrm{~s}), 1320(\mathrm{~s})$, 1245(s), 1142(s), 1036(m), 967(m), 851(s), 830(m), 756(m).

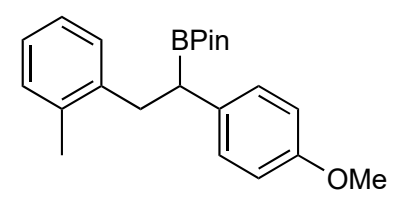

2-[1-(4-methoxyphenyl)-2-(2-methylphenyl)ethyl]-4,4,5,5-tetramethyl-1,3,2-dioxaborolane (37), compound was prepared according to reported procedure and was isolated as a clear colorless liquid (147 mg, 83\% yield). ${ }^{1} \mathrm{H}$ NMR (500 MHz, Chloroform- $d$ ) $\delta 7.48(\mathrm{~d}, J=8.4 \mathrm{~Hz}, 2 \mathrm{H}), 7.17$ $(\mathrm{d}, J=8.5 \mathrm{~Hz}, 2 \mathrm{H}), 7.09-7.03(\mathrm{~m}, 2 \mathrm{H}), 6.96(\mathrm{~d}, J=8.4 \mathrm{~Hz}, 2 \mathrm{H}), 6.81(\mathrm{~d}, J=8.3 \mathrm{~Hz}, 2 \mathrm{H}), 3.85$ (s, 3H), 3.12 (dd, $J=13.8,9.9 \mathrm{~Hz}, 1 \mathrm{H}), 2.87$ (dd, $J=13.8,6.4 \mathrm{~Hz}, 1 \mathrm{H}), 2.60$ (dd, $J=9.9,6.4 \mathrm{~Hz}$, $1 \mathrm{H}), 2.28(\mathrm{~s}, 3 \mathrm{H}), 1.13(\mathrm{~d}, J=6.7 \mathrm{~Hz}, 12 \mathrm{H}) .{ }^{13} \mathrm{C}$ NMR $(126 \mathrm{MHz}$, Chloroform- $d$ ) $\delta$ 140.1, 136.4, 135.0, 133.6, 130.1, 129.5, 128.0, 126.0, 125.6, 114.3, 83.5, 70.7, 55.9, 29.8, 24.8, 24.7, 19.5. ${ }^{11} \mathrm{~B}$ NMR (96 MHz, Chloroform- $d$ ) $\delta 32.8$. GCMS (EI) calculated for $[\mathrm{M}]+352.25$, found 352.3. FTIR (neat, $\left.\mathrm{cm}^{-1}\right): 2980(\mathrm{~s}), 2924(\mathrm{~s}), 2832(\mathrm{~m}), 1612(\mathrm{~s}), 1360(\mathrm{~m}), 1242(\mathrm{~s}), 1178(\mathrm{~m}), 1141(\mathrm{~m}), 1301(\mathrm{~s})$, 970(m), 841(m), 699(m).

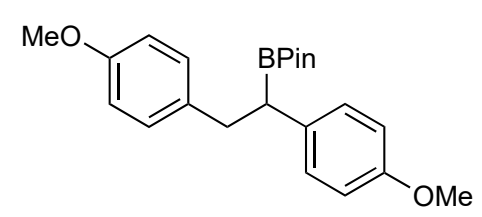

2-[1,2-bis(4-methoxyphenyl)ethyl]-4,4,5,5-tetramethyl-1,3,2-dioxaborolane (38), compound was prepared according to reported procedure and was purified by silica gel column chromatography $0-10 \%$ EtOAc in hexanes and was isolated as a clear colorless liquid (153 $\mathrm{mg}$, $81 \%$ yield). ${ }^{1} \mathrm{H}$ NMR (300 MHz, Chloroform- $d$ ) $\delta 7.12$ (dd, $\left.J=17.3,8.6 \mathrm{~Hz}, 4 \mathrm{H}\right), 6.79$ (dd, $J=$ $11.0,8.5 \mathrm{~Hz}, 4 \mathrm{H}), 3.77$ (d, $J=3.7 \mathrm{~Hz}, 6 \mathrm{H}), 3.07$ (dd, $J=13.5,9.6 \mathrm{~Hz}, 1 \mathrm{H}), 2.87$ (dd, $J=13.5,7.0$ $\mathrm{Hz}, 1 \mathrm{H}), 2.59(\mathrm{dd}, J=9.5,7.0 \mathrm{~Hz}, 1 \mathrm{H}), 1.13(\mathrm{~s}, 12 \mathrm{H}) .{ }^{13} \mathrm{C}$ NMR $(126 \mathrm{MHz}$, Chloroform- $d$ ) $\delta 157.8$, $157.5,134.7,134.1,129.9,129.4,113.8,113.5,83.4,55.3,55.2,38.3,24.9,24.7,24.7 .{ }^{11} \mathrm{~B}$ NMR (96 MHz, Chloroform- $d$ ) $\delta 32.5$. GCMS (EI) calculated for $[\mathrm{M}]+368.22$, found 368.2. FTIR (neat, $\left.\mathrm{cm}^{-1}\right): 2979(\mathrm{~s}), 2924(\mathrm{~s}), 2834(\mathrm{~m}), 1737(\mathrm{~m}), 1612(\mathrm{~s}), 1362(\mathrm{~m}), 1326(\mathrm{~m}), 1246(\mathrm{~s}), 1177(\mathrm{~m})$, 1142(m), 1307(s), 968(m), 841(m), 756(m).<smiles>FC(F)(F)c1ccc(CC(c2ccccc2)c2ccc(C(F)(F)F)cc2)cc1</smiles>

2-\{1,2-bis[4-(trifluoromethyl)phenyl]ethyl $\}-4,4,5,5$-tetramethyl-1,3,2-dioxaborolane (39), compound was prepared according to reported procedure using $\mathrm{IPrCuCl}$ instead of $\mathrm{IPrCuO} t-\mathrm{Bu}$ and $\mathrm{NaO} t$ - $\mathrm{Bu}$ instead of $\mathrm{KO} t$ - $\mathrm{Bu}$, and was purified by silica gel chromatography $0-100 \% \mathrm{DCM}$ in hexanes, and was isolated as a clear colorless liquid (147 mg, 83\% yield). ${ }^{1} \mathrm{H} \mathrm{NMR} \mathrm{(300} \mathrm{MHz,}$ Chloroform- $d$ ) $\delta 7.54-7.44(\mathrm{~m}, 1 \mathrm{H}), 7.39(\mathrm{~m}, 4 \mathrm{H}), 7.23-7.11(\mathrm{~m}, 4 \mathrm{H}), 3.12(\mathrm{dd}, J=13.7,8.9$ 
$\mathrm{Hz}, 1 \mathrm{H}), 2.93(\mathrm{dd}, J=20.6,13.1 \mathrm{~Hz}, 1 \mathrm{H}), 2.63(\mathrm{t}, J=8.2 \mathrm{~Hz}, 1 \mathrm{H}), 1.03(\mathrm{~s}, 12 \mathrm{H}) \cdot{ }^{13} \mathrm{C}$ NMR $(126$ MHz, Chloroform- $d$ ) $\delta 146.3,145.4,129.2,128.7,128.6$ (q, $J=311.2), 128.3$ (q, $J=32.3 \mathrm{~Hz}$ ), $128.1(\mathrm{q}, J=31.0 \mathrm{~Hz}), 125.5(\mathrm{q}, J=3.8 \mathrm{~Hz}), 125.2(\mathrm{q}, J=3.8 \mathrm{~Hz}), 124.9(\mathrm{q}, J=349.3 \mathrm{~Hz}), 84.0$, 38.3, 34.5, 24.7. ${ }^{19} \mathrm{~F}$ NMR (470 MHz, Chloroform- $d$ ) $\delta-65.2$, -65.3. ${ }^{11} \mathrm{~B}$ NMR $(96 \mathrm{MHz}$, Chloroform- $d$ ) $\delta$ 32.5. GCMS (EI) calculated for $[\mathrm{M}]+444.28$, found 444.3. FTIR (neat, $\mathrm{cm}^{-1}$ ): 2984(m), 2939(m), 1616(s), 2372(w), 1325(s), 1164(m), 1121(s), 1067(s), 1017(m), 848(m).

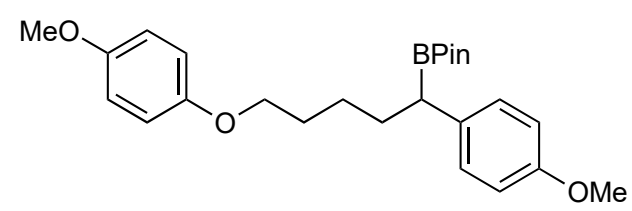

2-[5-(4-methoxyphenoxy)-1-(4-methoxyphenyl)pentyl]-4,4,5,5-tetramethyl-1,3,2-

dioxaborolane (40), compound was prepared according to reported procedure and was purified by silica gel chromatography $0-100 \%$ DCM in hexanes, and was isolated as a clear colorless liquid (179 mg, 84\% yield). ${ }^{1} \mathrm{H}$ NMR (300 MHz, Chloroform- $d$ ) $\delta 7.95$ (d, $\left.J=8.8 \mathrm{~Hz}, 2 \mathrm{H}\right), 6.93$ (d, $J=$ $8.8 \mathrm{~Hz}, 2 \mathrm{H}), 6.82(\mathrm{~s}, 4 \mathrm{H}), 3.96(\mathrm{t}, J=5.9 \mathrm{~Hz}, 2 \mathrm{H}), 3.87(\mathrm{~s}, 3 \mathrm{H}), 3.76(\mathrm{~s}, 3 \mathrm{H}), 2.23(\mathrm{t}, J=7.9 \mathrm{~Hz}$, $1 \mathrm{H}), 1.91-1.73(\mathrm{~m}, 1 \mathrm{H}), 1.71-1.51(\mathrm{~m}, 2 \mathrm{H}), 1.43-1.23(\mathrm{~m}, 2 \mathrm{H}), 1.19(\mathrm{~d}, J=6.0 \mathrm{~Hz}, 12 \mathrm{H}) .{ }^{13} \mathrm{C}$ NMR (126 MHz, Chloroform- $d$ ) $\delta$ 163.5, 153.9, 153.3, 130.4, 120.1, 115.6, 114.8, 113.8, 83.4, 68.4, 55.8, 55.6, 37.9, 29.1, 24.6, 24.2, 24.0, 21.3. ${ }^{11} \mathrm{~B}$ NMR (96 MHz, Chloroform- $d$ ) $\delta 32.4$. GCMS (EI) calculated for [M]+ 426.26, found 426.3. FTIR (neat, $\left.\mathrm{cm}^{-1}\right)$ : 3055(m), 2924(s), 2918(s), 2874(m), 1617(s), 1364(m), 1322(m), 1244(s), 1177(m), 1142(m), 1303(s), 966(m), 841(m), 756(m). 699(m).

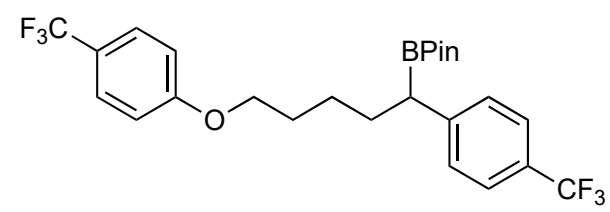

4,4,5,5-tetramethyl-2-\{5-[4-(trifluoromethyl)phenoxy]-1-[4-(trifluoromethyl)phenyl]pentyl\}1,3,2-dioxaborolane (41), compound was prepared according to reported procedure using $\mathrm{IPrCuCl}$ instead of $\mathrm{IPrCuO} t-\mathrm{Bu}$ and $\mathrm{NaO} t-\mathrm{Bu}$ instead of $\mathrm{KO} t-\mathrm{Bu}$, and was purified by silica gel chromatography $0-100 \%$ DCM in hexanes, and was isolated as a clear colorless liquid (171 $\mathrm{mg}$, $82 \%$ yield). ${ }^{1} \mathrm{H}$ NMR (300 MHz, Chloroform- $d$ ) $\delta 7.51$ (dd, $\left.J=8.6,3.7 \mathrm{~Hz}, 45 \mathrm{H}\right), 7.31$ (d, $J=8.1$ $\mathrm{Hz}, 2 \mathrm{H}), 6.90(\mathrm{~d}, J=8.6 \mathrm{~Hz}, 2 \mathrm{H}), 3.95(\mathrm{t}, J=6.3 \mathrm{~Hz}, 3 \mathrm{H}), 2.40(\mathrm{t}, J=7.9 \mathrm{~Hz}, 1 \mathrm{H}), 2.03-1.84$ $(\mathrm{m}, 1 \mathrm{H}), 1.77(\mathrm{~m}, 3 \mathrm{H}), 1.44(\mathrm{q}, J=7.7 \mathrm{~Hz}, 2 \mathrm{H}), 1.20(\mathrm{~d}, \mathrm{~J}=5.2 \mathrm{~Hz}, 12 \mathrm{H}) .{ }^{13} \mathrm{C}$ NMR $(126 \mathrm{MHz}$, Chloroform- $d$ ) $\delta 161.5,147.5,128.6,127.6(\mathrm{q}, \mathrm{J}=32.8), 126.8(\mathrm{q}, \mathrm{J}=3.8 \mathrm{~Hz}), 125.9(\mathrm{q}, \mathrm{J}=277.1$ $\mathrm{Hz}), 125.2(\mathrm{q}, \mathrm{J}=3.8 \mathrm{~Hz}), 124.5(\mathrm{q}, \mathrm{J}=272.2 \mathrm{~Hz}), 122.6(\mathrm{q}, \mathrm{J}=32.8 \mathrm{~Hz}), 114.4$, 83.6, 67.9, 32.0, 29.0, 25.5, 24.6, 24.5. ${ }^{19} \mathrm{~F}$ NMR (470 MHz, Chloroform- $d$ ) $\delta-64.3,-65.0 .{ }^{11} \mathrm{~B}$ NMR $(96 \mathrm{MHz}$, Chloroform- $d$ ) $\delta$ 31.7. GCMS (EI) calculated for $[\mathrm{M}]+502.21$, found 502.2. FTIR (neat, $\mathrm{cm}^{-1}$ ): 3054(m), 2980(s), 2937(s), 2872(m), 1617(s), 1372(m), 1326(s), 1259(m), 1162(m), 1111(s), 1068(m), 836(m).

\section{Aryl Bromide Starting Materials}<smiles>COc1ccc(Br)cc1</smiles> 
4-bromoanisole (2) was purchased from Millipore Sigma and distilled over calcium hydride under reduced pressure before use.<smiles>FC(F)(F)c1ccc(Br)cc1</smiles>

4-bromobenzotrifluoride (S1) was purchased from Millipore Sigma and distilled over calcium hydride under reduced pressure before use.<smiles>Fc1ccc(Br)cc1</smiles>

1-bromo-4-fluorobenzene (S2) was purchased from Millipore Sigma and distilled over calcium hydride under reduced pressure before use.<smiles>Clc1ccc(Br)cc1</smiles>

1-bromo-4-chlorobenzene (S3) was purchased from Millipore Sigma and distilled over calcium hydride under reduced pressure before use.<smiles>CN(C)c1ccc(Br)cc1</smiles>

4-bromo-N,N-dimethylaniline (S4) was purchased from Oakwood Chemicals and used without purification.<smiles>N#Cc1ccc(Br)cc1</smiles>

4-bromobenzonitrile (S5) was purchased from Millipore Sigma and used without purification.<smiles>Cc1ccc(Br)cc1</smiles>

4-bromotoluene (S6) was purchased form Alfa Aesar and using without purification.<smiles>Cc1cccc(Br)c1</smiles>

3-bromotoluene (S7) was purchased form TCI America and distilled over calcium hydride under reduced pressure before use.<smiles>COc1ccccc1Br</smiles> 
2-bromoanisole (S8) was purchased from Millipore Sigma and distilled over calcium hydride under reduced pressure before use.<smiles>Brc1ccc2ccccc2c1</smiles>

2-bromonaphthalene (S9) was purchased from Ark-Pharm and used without purification.<smiles>Brc1ccc2occc2c1</smiles>

2-bromobenzofuran (S10) was purchased from Ark-Pharm and used without further purification<smiles>Brc1ccc(C2OCCO2)cc1</smiles>

1-bromo-4-phenyl-1,3-dioxolane (S11) was purchased from Ark-Pharm and distilled over calcium hydride under reduced pressure before use.<smiles>Brc1ccco1</smiles>

2-bromofuran (S12) was purchased from TCI chemicals and distilled over calcium hydride under reduced pressure before used.<smiles>Brc1cccs1</smiles>

2-bromothiophene (S13) was purchased from Combi-Blocks and distilled over calcium hydride under reduced pressure before use.<smiles>Brc1cnc2ccccc2c1</smiles>

3-bromoquinoline (S14) was purchased form Millipore Sigma and distilled over calcium hydride under reduced pressure before use.<smiles>C=Cc1ccc(Br)cc1</smiles>

4-bromostyrene (S15) was purchased from TCI America and degassed before use.<smiles>Brc1ccc2c(c1)OCO2</smiles>

4-Bromo-1,2-(methylenedioxy)benzene (S16) was purchased from Millipore Sigma and distill over calcium hydride under reduced pressure before use. 


\section{Alkyne Starting Materials}<smiles>C#CCCCc1ccccc1</smiles>

5-phenyl-1-pentyne (1) was purchased from GFS Chemical and distilled over calcium hydride under reduced pressure before use.<smiles>C#CCCC[NH3+]</smiles>

hex-5-ynenitrile (S17) was purchased from Oakwood Chemical and distilled over calcium hydride under reduced pressure before use.<smiles>C#CCCCOc1ccccc1</smiles>

(pent-4-yn-1-yloxy)benzene (S18) was prepared according to a known procedure and has been previously characterized. ${ }^{1}$<smiles>C#CC[14CH2]C1CO1</smiles>

2-(dec-9-yn-1-yl)oxirane (S19) was prepared according to a known procedure and has been previously characterized. $^{2}$<smiles>C#CCCCOC1CCCCO1</smiles>

2-(pent-4-yn-1-yloxy)tetrahydra-2H-pyran (S20) has been previously characterized and spectral data match literature values. ${ }^{3}$<smiles>C#CCCCCCl</smiles>

6-chlorohex-1-yne (S21) was purchased from TCI America and distilled over calcium hydride under reduced pressure before use.<smiles>C#CCCCCCBr</smiles>

7-bromohept-1-yne (S22) was prepared according to a known procedure and has been previously characterized. ${ }^{4}$<smiles>C#CCCCCOC(C)(C)C</smiles>

tert-butyldimethyl(pent-4-yn-1-yloxy)silane (S23) was prepared according to a known procedure and has been previously characterized. ${ }^{5}$<smiles>C#CC(CCCCC)O[Sb]</smiles>

tert-butyldimethyl(oct-1-yn-3-yloxy)silane (S24) was prepared according to a known literature procedure and has been previously characterized. ${ }^{6}$ 
<smiles>C#CC1CCCCC1</smiles>

ethynylcyclohexane (S25) was purchased from Milipore Sigma and distilled over calcium hydride under reduced pressure before use.<smiles>C#CC(C)(C)C</smiles>

3,3-dimethylbut-1-yne (S26) was purchased from Milipore Sigma and distilled over calcium hydride under reduced pressure before use.<smiles>C#CCCC1OCCO1</smiles>

2-(but-3-yn-1-yl)-1,3-dioxolane (S27) was synthesized according to a known literature procedure and has been previously characterized. ${ }^{7}$<smiles>C#CCCCOC(=O)C(C)(C)C</smiles>

pent-4-yn-1-yl 2,2-dimethylpropanoate (S28) was synthesized according to a known literature procedure and has been previously characterized. ${ }^{8}$<smiles>C#Cc1ccccc1C</smiles>

1-ethynyl-2-methylbenzene (S29) was purchased from Millipore Sigma and distilled over calcium hydride under reduced pressure before use.<smiles>C#Cc1ccc(OC)cc1</smiles>

1-ethynyl-4-methoxybenzene (S30) was purchase from Millipore Sigma and distilled over calcium hydride under reduced pressure before use.<smiles>C#Cc1ccc(C(F)(F)F)cc1</smiles>

1-ethynyl-4-(trifluoromethyl)benzene (S31) was purchased from Millipore Sigma and distilled over calcium hydride under reduced pressure before use.<smiles>C#CCCCOc1ccc(OC)cc1</smiles>

1-methoxy-4-(pent-4-yn-1-yloxy)benzene (S32) was synthesized according to a known literature procedure and has been previously characterized. ${ }^{9}$ 
<smiles>C#CCCCOc1ccc(C(F)(F)F)cc1</smiles>

1-(pent-4-yn-1-yloxy)-4-(trifluoromethyl)benzene ( $\mathbf{S 3 3}$ ) was synthesized according to a modified procedure. ${ }^{9}{ }^{1} \mathrm{H}$ NMR $(300 \mathrm{MHz}$, Chloroform- $d$ ) $\delta 7.60-7.44(\mathrm{~m}, 2 \mathrm{H}), 6.96(\mathrm{~d}, J=8.5$ $\mathrm{Hz}, 2 \mathrm{H}), 4.12(\mathrm{t}, J=6.1 \mathrm{~Hz}, 2 \mathrm{H}), 2.42(\mathrm{td}, J=6.9,2.7 \mathrm{~Hz}, 2 \mathrm{H}), 2.10-1.92(\mathrm{~m}, 3 \mathrm{H}) .{ }^{13} \mathrm{C}$ NMR $\left(126 \mathrm{MHz}, \mathrm{CDCl}_{3}\right) \delta 161.6,127.0(\mathrm{q}, J=3.9 \mathrm{~Hz}), 124.7(\mathrm{q}, J=270.9 \mathrm{~Hz}), 123.0(\mathrm{q}, J=32.6 \mathrm{~Hz})$, 114.6, 83.2, 69.2, 66.5, 28.1, 15.2. ${ }^{19} \mathrm{~F} \mathrm{NMR}\left(470 \mathrm{MHz}, \mathrm{CDCl}_{3}\right) \delta-64.4$.<smiles>CC(C)c1cccc(C(C)C)c1N1C=CN(c2c(C(C)C)cccc2C(C)C)C1C(Cl)Cl</smiles>

\section{\{1-[2,6-bis(propan-2-yl)phenyl]-3-[2-methyl-6-(propan-2-yl)phenyl]-2,3-dihydro-1H-}

imidazol-2-yl\}(chloro)copper (S34), was synthesized according to a known literature procedure and has been previously characterized. ${ }^{10}$<smiles>CCCCC1N(c2c(F)cccc2C(C)C)C=CN1c1c(F)cccc1C(C)C</smiles>

\section{\{1-[2,6-bis(propan-2-yl)phenyl]-3-[2-methyl-6-(propan-2-yl)phenyl]-2,3-dihydro-1H-}

imidazol-2-yl\}(tert-butoxy)copper (S35), was synthesized according to a known literature procedure and has been previously characterized. ${ }^{11}$

\section{Mechanistic Experiments (Scheme 3)}

\section{Analysis of Potential Catalytic Intermediates}

Alkenyl Bpin (5)

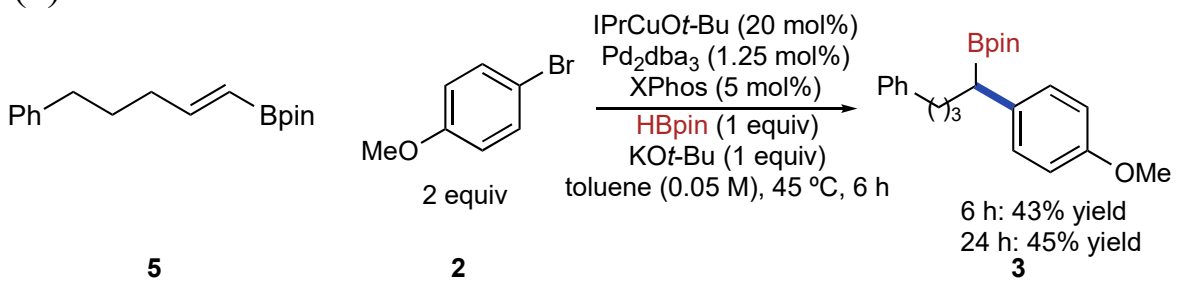

In a nitrogen-filled glovebox, a scintillation vial was charged with a stir bar, $\mathrm{KO} t-\mathrm{Bu}(6.1 \mathrm{mg}$, $0.050 \mathrm{mmol}, 1$ equiv), $\mathrm{IPrCuOt}$-Bu (5.3 mg, $0.01 \mathrm{mmol}, 0.20$ equiv), HBpin ( $6.4 \mathrm{mg}, 0.05 \mathrm{mmol}$, 1.0 equiv), toluene $(1 \mathrm{~mL})$ and alkenyl Bpin $(5)(13.6 \mathrm{mg}, 0.05 \mathrm{mmol}, 1.0$ equiv). The reaction mixture was stirred at $45^{\circ} \mathrm{C}$ for $1 \mathrm{~min}$. To this reaction mixture was added $\mathrm{Pd}_{2} \mathrm{dba}_{3}(0.6 \mathrm{mg}, 0.001$ mmol, 0.0125 equiv), XPhos ( $1.2 \mathrm{mg}, 0.0025$ equiv) and 4-bromoanisole (2) $(18.7 \mathrm{mg}, 0.1 \mathrm{mmol}$, 2.0 equiv) and the reaction mixture was vigorously stirred at $45^{\circ} \mathrm{C}$. After both $6 \mathrm{~h}$ and $24 \mathrm{~h}$ a 60 $\mu \mathrm{L}$ was taken and pushed through a plug of silica with $1.5 \mathrm{~mL}$ EtOAc and analyzed by GC. 
E-styrene (6)

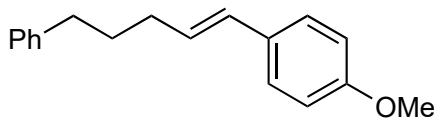

6

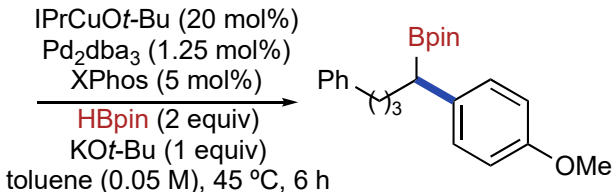

3

$6 \mathrm{~h}:<5 \%$ yield $24 \mathrm{~h}$ : $<5 \%$ yield

In a nitrogen-filled glovebox, a scintillation vial was charged with a stir bar, $\mathrm{KO} t$ - $\mathrm{Bu}(12.2 \mathrm{mg}$, $0.10 \mathrm{mmol}, 2$ equiv), $\mathrm{IPrCuOt}$-Bu (5.3 mg, $0.01 \mathrm{mmol}, 0.20$ equiv), HBpin ( $12.8 \mathrm{mg}, 0.10 \mathrm{mmol}$, 2.0 equiv), toluene ( $1 \mathrm{~mL}$ ) and $E$-styrene (6) $(12.6 \mathrm{mg}, 0.05 \mathrm{mmol}, 1.0$ equiv). The reaction mixture was stirred at $45^{\circ} \mathrm{C}$ for $1 \mathrm{~min}$. To this reaction mixture was added $\mathrm{Pd}_{2} \mathrm{dba}_{3}(0.6 \mathrm{mg}, 0.001 \mathrm{mmol}$, 0.0125 equiv) and XPhos ( $1.2 \mathrm{mg}, 0.0025$ equiv) and the reaction mixture was vigorously stirred at $45^{\circ} \mathrm{C}$. After both $6 \mathrm{~h}$ and $24 \mathrm{~h}$ a $60 \mu \mathrm{L}$ was taken and pushed through a plug of silica with 1.5 $\mathrm{mL}$ EtOAc and analyzed by GC.

Alkyl diboronate (7)
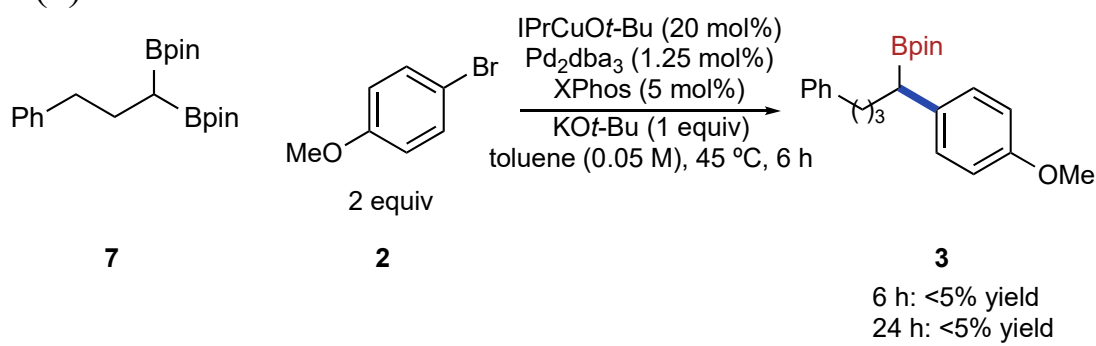

In a nitrogen-filled glovebox, a scintillation vial was charged with a stir bar, $\mathrm{KO} t$ - $\mathrm{Bu}(6.1 \mathrm{mg}, 0.05$ mmol, 1 equiv), $\mathrm{IPrCuOt}$ - $\mathrm{Bu}(5.3 \mathrm{mg}, 0.01 \mathrm{mmol}, 0.20$ equiv), toluene $(1 \mathrm{~mL})$ and alkyl diboronate (7) $\left(18.6 \mathrm{mg}, 0.05 \mathrm{mmol}, 1.0\right.$ equiv). The reaction mixture was stirred at $45^{\circ} \mathrm{C}$ for $1 \mathrm{~min}$. To this reaction mixture was added $\mathrm{Pd}_{2} \mathrm{dba}_{3}(0.6 \mathrm{mg}, 0.001 \mathrm{mmol}, 0.0125$ equiv), XPhos ( $1.2 \mathrm{mg}, 0.0025$ mmol, 0.05 equiv) and 4-bromoanisole (2) $(18.7 \mathrm{mg}, 0.1 \mathrm{mmol}, 2.0$ equiv) and the reaction mixture was vigorously stirred at $45^{\circ} \mathrm{C}$. After $6 \mathrm{~h}$ and $24 \mathrm{~h} \mathrm{a} 60 \mu \mathrm{L}$ was taken and pushed through a plug of silica with $1.5 \mathrm{~mL}$ EtOAc and analyzed by GC.

\section{Synthesis of Heterobimetallic Intermediate (42)}

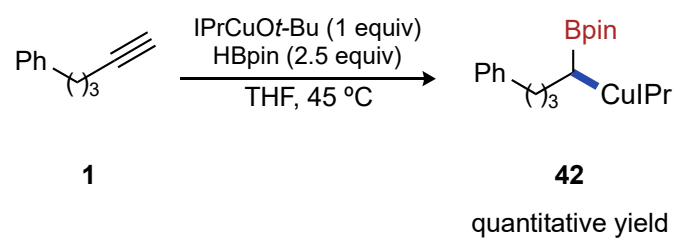

In a nitrogen-filled glovebox, a scintillation vial was charged with a stir bar, $\mathrm{IPrCuO} t$ - $\mathrm{Bu}(131.6$ $\mathrm{mg}, 0.250 \mathrm{mmol}, 1.0$ equiv) and THF $(1 \mathrm{~mL})$. To this reaction mixture was added HBpin $(80.0$ $\mathrm{mg}, 0.625 \mathrm{mmol}, 2.50$ equiv) and 5-phenyl-1-pentyne (1) ( $37.9 \mathrm{mg}, 0.263 \mathrm{mmol}, 1.05$ equiv) and the reaction mixture was vigorously stirred at $45{ }^{\circ} \mathrm{C}$ until the orange color had disappeared. The reaction was removed from hot plate and pentane was carefully layered over the reaction mixture 
and then placed in a $-35{ }^{\circ} \mathrm{C}$ freezer overnight. Filtration with cold pentane yielded the desired product as a white solid ( $180.3 \mathrm{mg}, 99 \%$ yield).

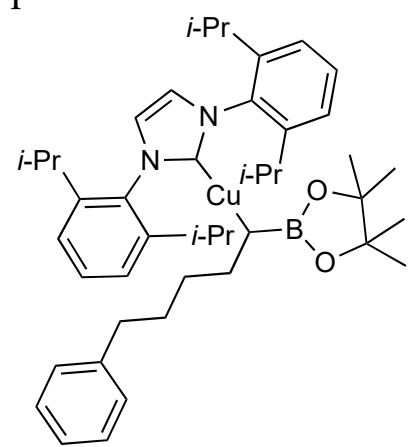

\{1,3-bis[2,6-bis(propan-2-yl)phenyl]-2,3-dihydro-1H-imidazol-2-yl\}[5-phenyl-1-(4,4,5,5tetramethyl-1,3,2-dioxaborolan-2-yl)pentyl]copper (42). ${ }^{1} \mathrm{H}$ NMR $(500 \mathrm{MHz}$, Chloroform- $d$ ) $\delta$ $7.31-7.14(\mathrm{~m}, 15 \mathrm{H}), 6.30(\mathrm{~s}, 2 \mathrm{H}), 2.71-2.60(\mathrm{~m}, 6 \mathrm{H}), 2.20-2.11(\mathrm{~m}, 1 \mathrm{H}), 1.90-1.86(\mathrm{~m}, 1 \mathrm{H})$, $1.69(\mathrm{p}, J=7.7 \mathrm{~Hz}, 2 \mathrm{H}), 1.52(\mathrm{t}, J=6.0 \mathrm{~Hz}, 12 \mathrm{H}), 1.16(\mathrm{~m}, 12 \mathrm{H}), 1.12(\mathrm{~s}, 6 \mathrm{H}), 1.08(\mathrm{~s}, 6 \mathrm{H}) .{ }^{13} \mathrm{C}$ NMR (126 MHz, Chloroform- $d$ ) $\delta 145.9,145.9,135.5,130.4,128.9,125.4,124.2,124.2,122.2$, 120.3, 117.6, 79.7, 37.7, 36.7, 32.7, 29.1, 29.0, 28.8, 25.5, 25.3, 25.1, 23.8. ${ }^{11}$ B NMR (96 MHz, Chloroform- $d$ ) $\delta$ 28.4. LCMS (ESI) calculated for [M+]: 726.3, found: 726.7 .

Palladium-catalyzed Cross Coupling of Bimetallic Intermediate (42) and ArBr (2)

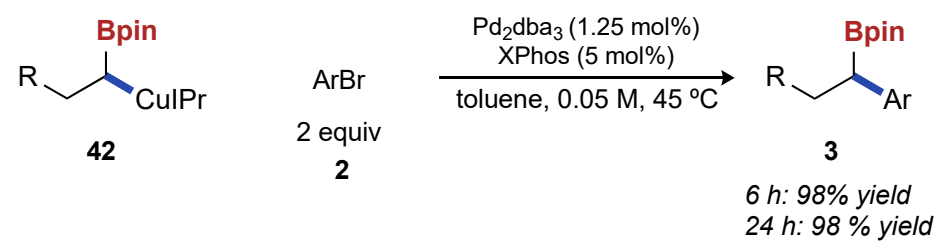

In a nitrogen-filled glovebox, a scintillation vial was charged with a stir bar, hetereobimetallic complex (38) (36.3 mg, $0.05 \mathrm{mmol}, 1.0$ equiv), XPhos ( $1.2 \mathrm{mg}, 0.0025 \mathrm{mmol}, 0.05$ equiv) and 4bromoanisole (18.7 $\mathrm{mg}, 0.1 \mathrm{mmol}, 2.0$ equiv) and the reaction mixture was vigorously stirred at $45^{\circ} \mathrm{C}$. After $6 \mathrm{~h}$ and $24 \mathrm{~h}$ a $60 \mu \mathrm{L}$ was taken and pushed through a plug of silica with $1.5 \mathrm{~mL}$ EtOAc and analyzed by GC.

\section{Characterization of Mechanistic Compounds}<smiles>C(=CCc1ccccc1)CCCc1ccccc1</smiles>

4,4,5,5-tetramethyl-2-[(1E)-5-phenylpent-1-en-1-yl]-1,3,2-dioxaborolane (5), compound was synthesized according to known literature procedure and has been previously characterized. ${ }^{12}{ }^{1} \mathrm{H}$ NMR (300 MHz, Chloroform- $d$ ) $\delta 7.33-7.22(\mathrm{~m}, 3 \mathrm{H}), 7.21-7.12(\mathrm{~m}, 3 \mathrm{H}), 6.65$ (dt, $J=18.0,6.4$ $\mathrm{Hz}, 1 \mathrm{H}), 5.46(\mathrm{~d}, J=18.0 \mathrm{~Hz}, 1 \mathrm{H}), 2.71-2.56(\mathrm{~m}, 2 \mathrm{H}), 2.28-2.12(\mathrm{~m}, 2 \mathrm{H}), 1.76(\mathrm{~m}, 2 \mathrm{H}), 1.27$ $(\mathrm{s}, 12 \mathrm{H})$.

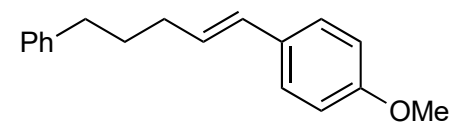

1-methoxy-4-[(1E)-5-phenylpent-1-en-1-yl]benzene (6), compound was synthesized according to known literature procedure and has been previously characterized. ${ }^{13}{ }^{1} \mathrm{H} \mathrm{NMR}(300 \mathrm{MHz}$, 
Chloroform- $d$ ) $\delta 7.37-7.12(\mathrm{~m}, 8 \mathrm{H}), 6.84(\mathrm{~d}, J=8.7 \mathrm{~Hz}, 2 \mathrm{H}), 6.34(\mathrm{~d}, J=15.8 \mathrm{~Hz}, 1 \mathrm{H}), 6.09(\mathrm{dt}$, $J=15.8,6.8 \mathrm{~Hz}, 1 \mathrm{H}), 3.80(\mathrm{~s}, 3 \mathrm{H}), 2.67(\mathrm{t}, J=7.5 \mathrm{~Hz}, 2 \mathrm{H}), 2.30-2.18(\mathrm{~m}, 2 \mathrm{H}), 1.80(\mathrm{p}, J=7.5$ $\mathrm{Hz}, 2 \mathrm{H})$.<smiles>[B]C(CCCc1ccccc1)CCc1ccccc1Br</smiles>

4,4,5,5-tetramethyl-2-[5-phenyl-1-(4,4,5,5-tetramethyl-1,3,2-dioxaborolan-2-yl)propyl]-1,3,2dioxaborolane (7), compound was synthesized according to known literature procedure and has been previously characterized. ${ }^{14}{ }^{1} \mathrm{H}$ NMR $(300 \mathrm{MHz}$, Chloroform- $d) \delta 7.41-7.05(\mathrm{~m}, 7 \mathrm{H}), 2.70$ $-2.53(\mathrm{~m}, 2 \mathrm{H}), 1.85(\mathrm{q}, J=7.8 \mathrm{~Hz}, 2 \mathrm{H}), 1.23(\mathrm{~s}, 25 \mathrm{H}), 0.81(\mathrm{t}, J=7.9 \mathrm{~Hz}, 1 \mathrm{H})$.

\section{Evaluation of Intermediates in Catalytic Reaction}

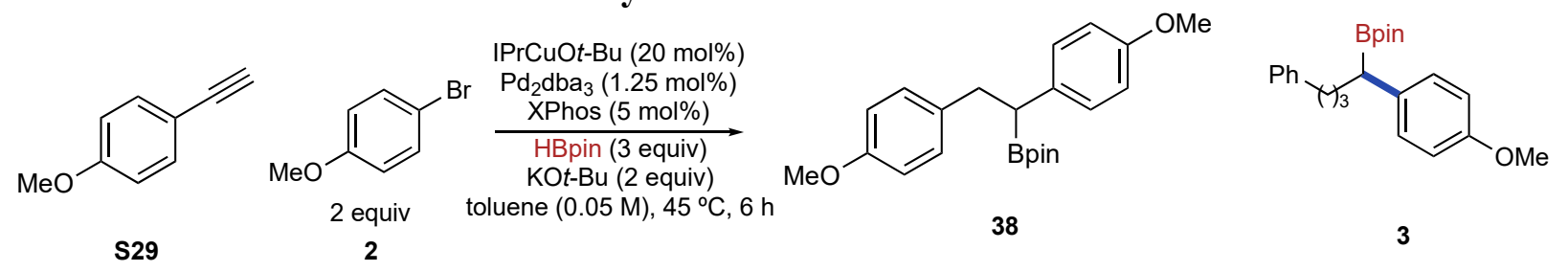

Intermediate Additives<smiles>[R]C=Cc1ccccc1</smiles><smiles>[R]C=Cc1ccc(OC)cc1</smiles><smiles>[R]CC([10BH2])[18OH]</smiles>

In a nitrogen-filled glovebox, a scintillation vial was charged with a stir bar, $\mathrm{KO} t$-Bu (12.2 mg, $0.10 \mathrm{mmol}, 2.0$ equiv), $\mathrm{IPrCuOt}$-Bu (5.3 mg, $0.01 \mathrm{mmol}, 0.20$ equiv), HBpin (19.2 mg, 0.15 mmol, 3.0 equiv), toluene (1 mL) and 4-ethynylanisole (S29) $(6.6 \mathrm{mg}, 0.05 \mathrm{mmol}, 1.0$ equiv). The reaction mixture was stirred at $45{ }^{\circ} \mathrm{C}$ for $1 \mathrm{~min}$. To this reaction mixture was added $\mathrm{Pd}_{2} \mathrm{dba}_{3}$ (0.6 mg, $0.001 \mathrm{mmol}, 0.0125$ equiv), XPhos (1.2 mg, 0.0025 equiv) and 4-bromoanisole (2) (18.7 mg, $0.1 \mathrm{mmol}, 2.0$ equiv) and either alkenyl Bpin (5), E-styrene (6) or alkyl diboronate (7) ( $0.05 \mathrm{mmol}, 1.0$ equiv). The reaction mixture was vigorously stirred at $45^{\circ} \mathrm{C}$. After $6 \mathrm{~h} \mathrm{a} 60 \mu \mathrm{L}$ was taken and pushed through a plug of silica with $1.5 \mathrm{~mL}$ EtOAc and analyzed by GC.

\begin{tabular}{cccc} 
Entry & Additive & Yield 38 & Yield 3 \\
\hline 1 & Alkenyl Bpin (5) & 28 & 33 \\
2 & $E$-styrene (6) & 31 & 0 \\
3 & Alkyl diboronate (7) & 42 & 0
\end{tabular}

\section{References}

(1) Jin, L. et al. N-Heterocyclic carbene copper-catalyzed direct alkylation of terminal alkynes with non-activated alkyl triflates. Chem. Commun. 53, 4124-4127 (2017).

(2) Uehling, M. R., Rucker, R. P. \& Lalic, G. Catalytic Anti-Markovnikov Hydrobromination of Alkynes. J. Am. Chem. Soc. 136, 8799-8803 (2014).

(3) Mostafa, M. A. B., McMillan, A. E. \& Sutherland, A. Structural diversification of the aminobicyclo[4.3.0]nonane skeleton using alkynylsilyl-derived allylic trichloroacetimidates. Org. Biomol. Chem. 15, 3035-3045 (2017). 
(4) Mailig, M., Hazra, A., Armstrong, M. K. \& Lalic, G. Catalytic Anti-Markovnikov Hydroallylation of Terminal and Functionalized Internal Alkynes: Synthesis of Skipped Dienes and Trisubstituted Alkenes. J. Am. Chem. Soc. 139, 6969-6977 (2017).

(5) Balas, L. et al. Regiocontrolled syntheses of FAHFAs and LC-MS/MS differentiation of regioisomers. Org. Biomol. Chem. 14, 9012-9020 (2016).

(6) Choppin, S., Barbarotto, M., Obringer, M. \& Colobert, F. Synthesis of an Advanced Fragment of (+)-Trienomycinol. Synthesis 48, 3263-3271 (2016).

(7) Abdel Ghani, S. B. et al. Total Synthesis and Stereochemical Assignment of cisUvariamicin I and cis-Reticulatacin. J. Org. Chem. 74, 6924-6928 (2009).

(8) Hurtak, J. A. \& McDonald, F. E. Synthesis of the ABC Substructure of Brevenal by Sequential exo -Mode Oxacyclizations of Acyclic Polyene Precursors. Org. Lett. 19, 6036-6039 (2017).

(9) Lee, M., Nguyen, M., Brandt, C., Kaminsky, W. \& Lalic, G. Catalytic Hydroalkylation of Allenes. Angew. Chem. Int. Ed. 56, 15703-15707 (2017).

(10) Santoro, O., Collado, A., Slawin, A. M. Z., Nolan, S. P. \& Cazin, C. S. J. A general synthetic route to $[\mathrm{Cu}(\mathrm{X})(\mathrm{NHC})](\mathrm{NHC}=\mathrm{N}$-heterocyclic carbene, $\mathrm{X}=\mathrm{Cl}, \mathrm{Br}, \mathrm{I})$ complexes. Chem. Commun. 49, 10483 (2013).

(11) Mankad, N. P., Laitar, D. S. \& Sadighi, J. P. Synthesis, Structure, and Alkyne Reactivity of a Dimeric (Carbene)copper(I) Hydride. Organometallics 23, 3369-3371 (2004).

(12) Pereira, S. \& Srebnik, M. Hydroboration of Alkynes with Pinacolborane Catalyzed by HZrCp2Cl. Organometallics 14, 3127-3128 (1995).

(13) Armstrong, M. K., Goodstein, M. B. \& Lalic, G. Diastereodivergent Reductive Cross Coupling of Alkynes through Tandem Catalysis: $Z$ - and $E$-Selective Hydroarylation of Terminal Alkynes. J. Am. Chem. Soc. 140, 10233-10241 (2018).

(14) Sun, C., Potter, B. \& Morken, J. P. A Catalytic Enantiotopic-Group-Selective Suzuki Reaction for the Construction of Chiral Organoboronates. J. Am. Chem. Soc. 136, 65346537 (2014).

\section{Spectral Data}



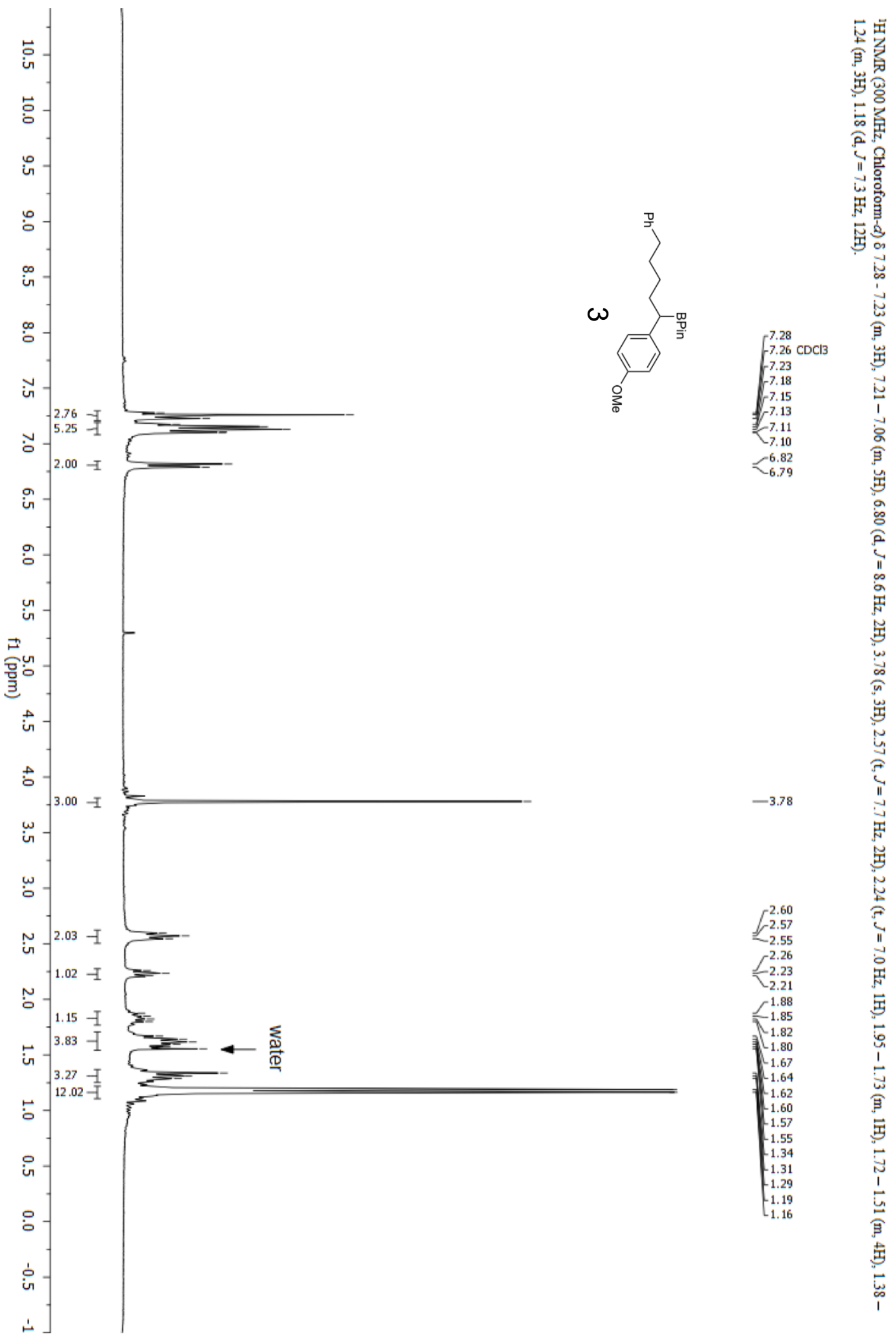

S29 

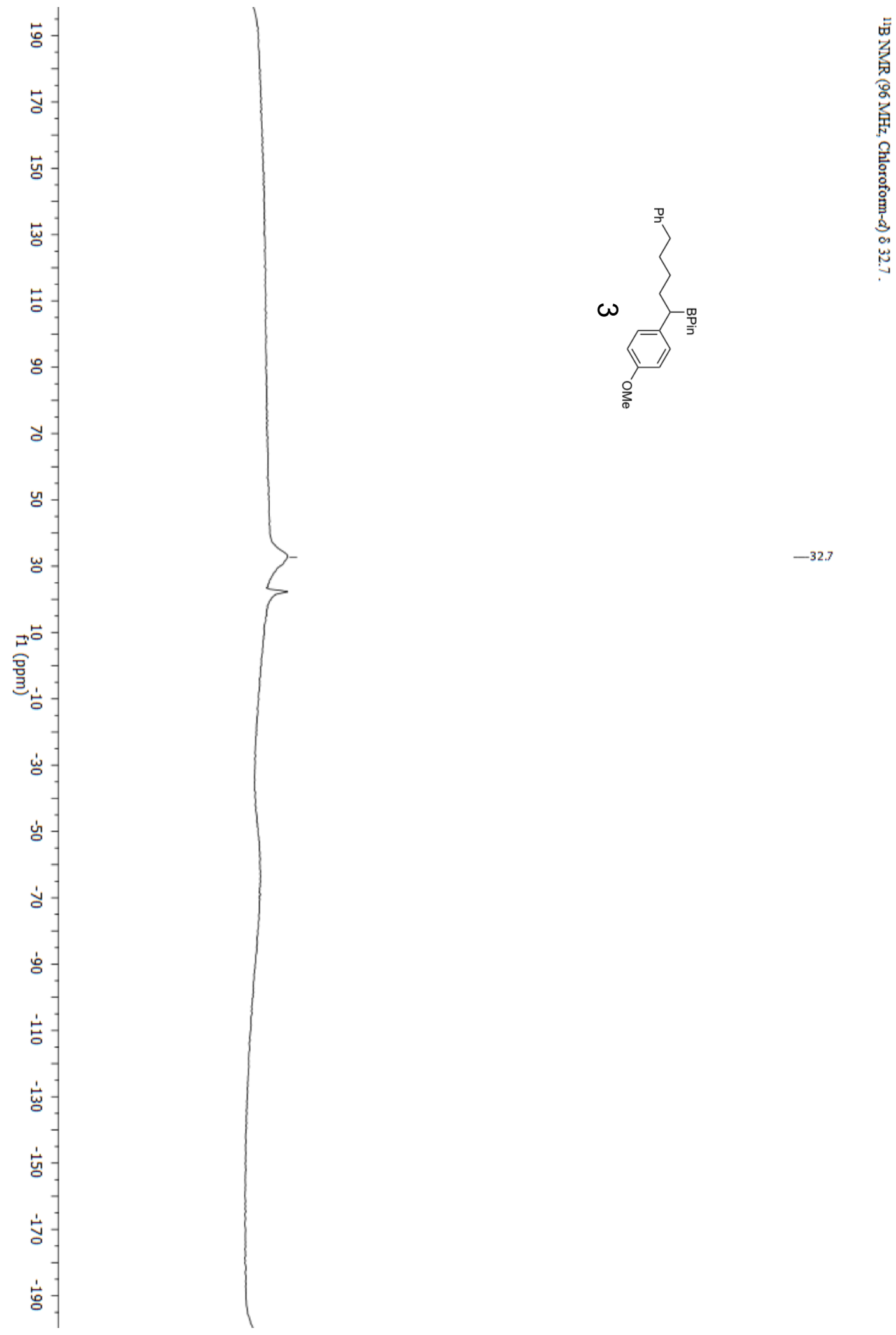


$$
\mid
$$




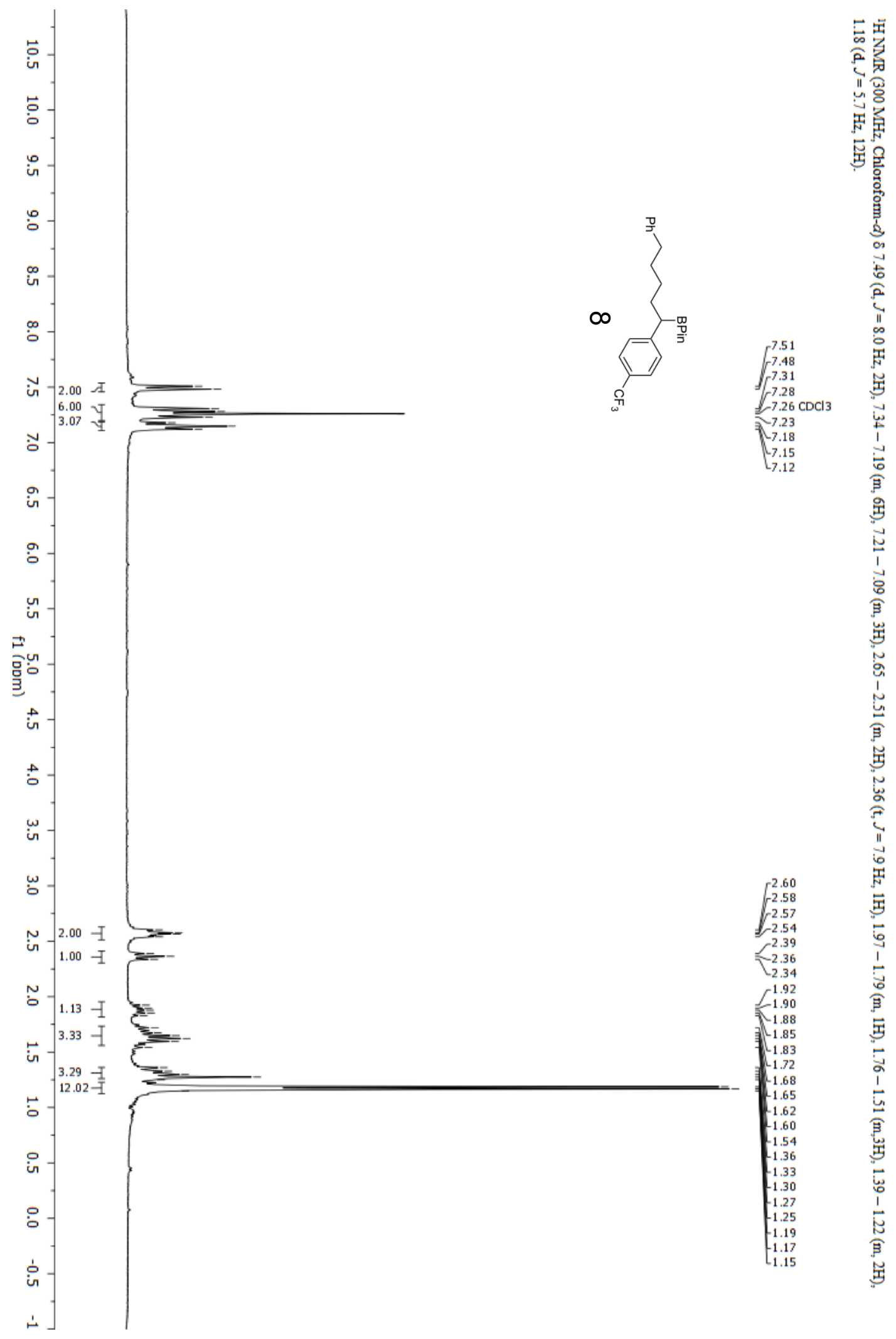




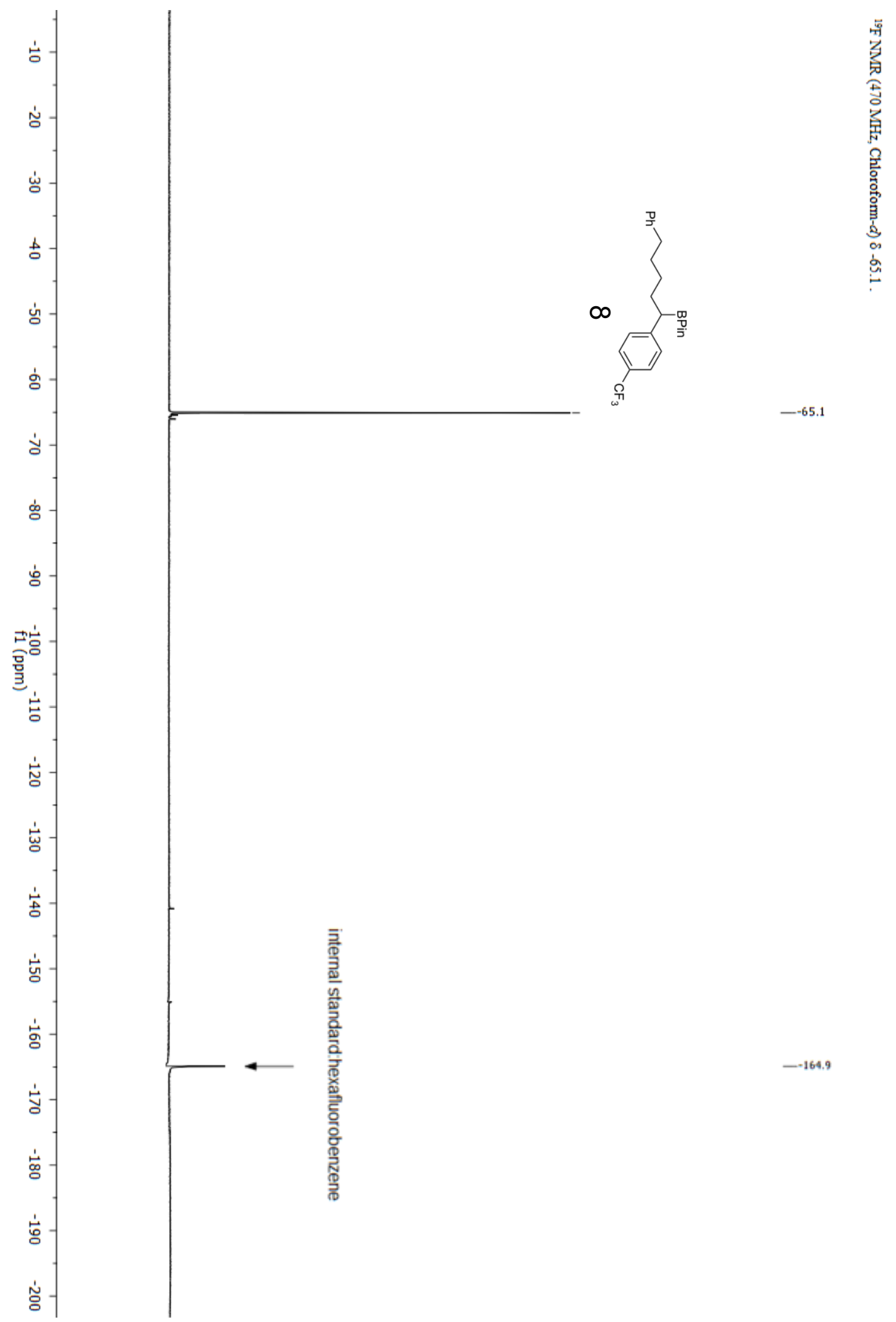



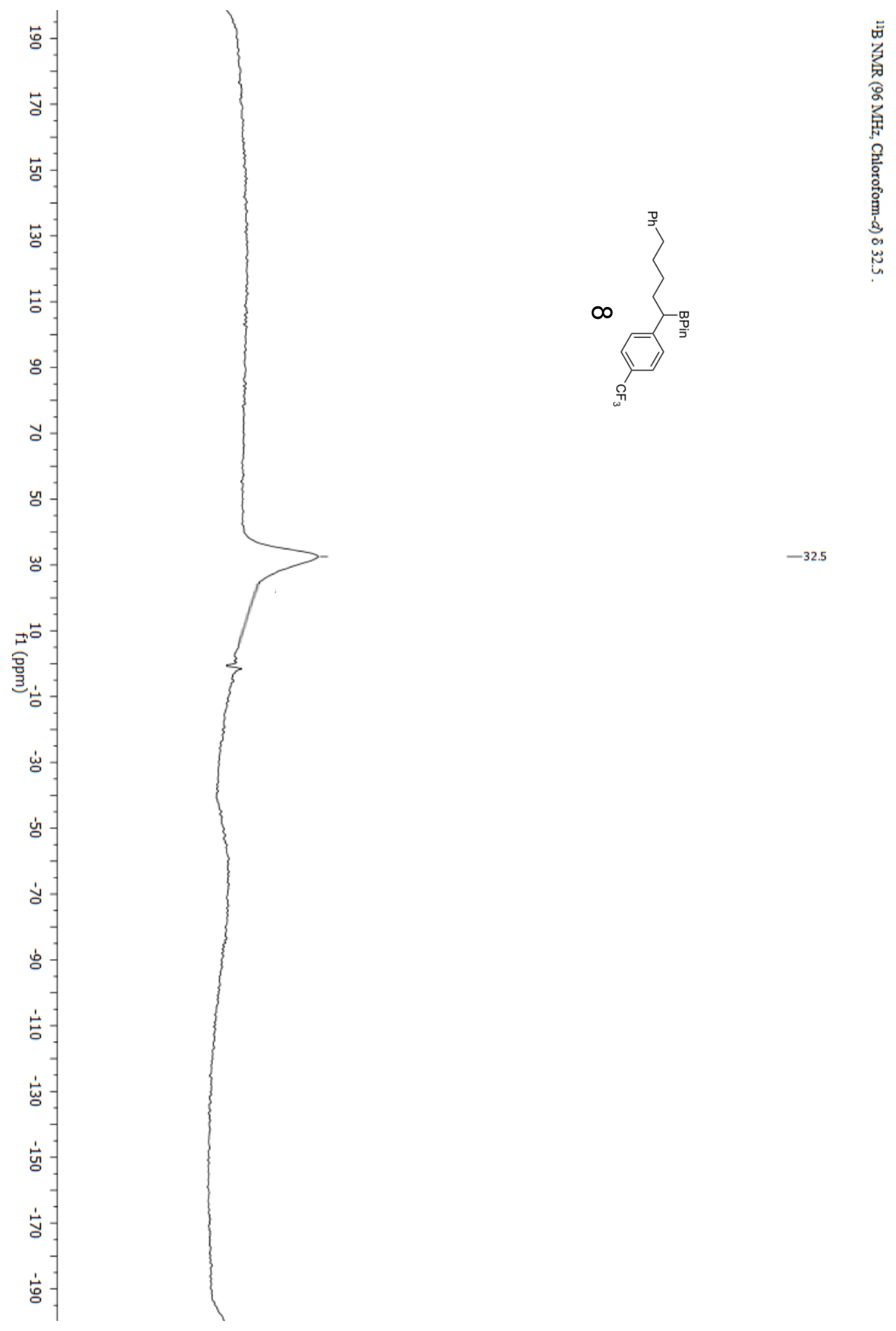

$\infty$

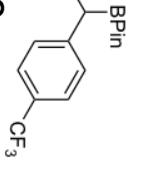

$-32.5$ 


$$
\text { F! }
$$




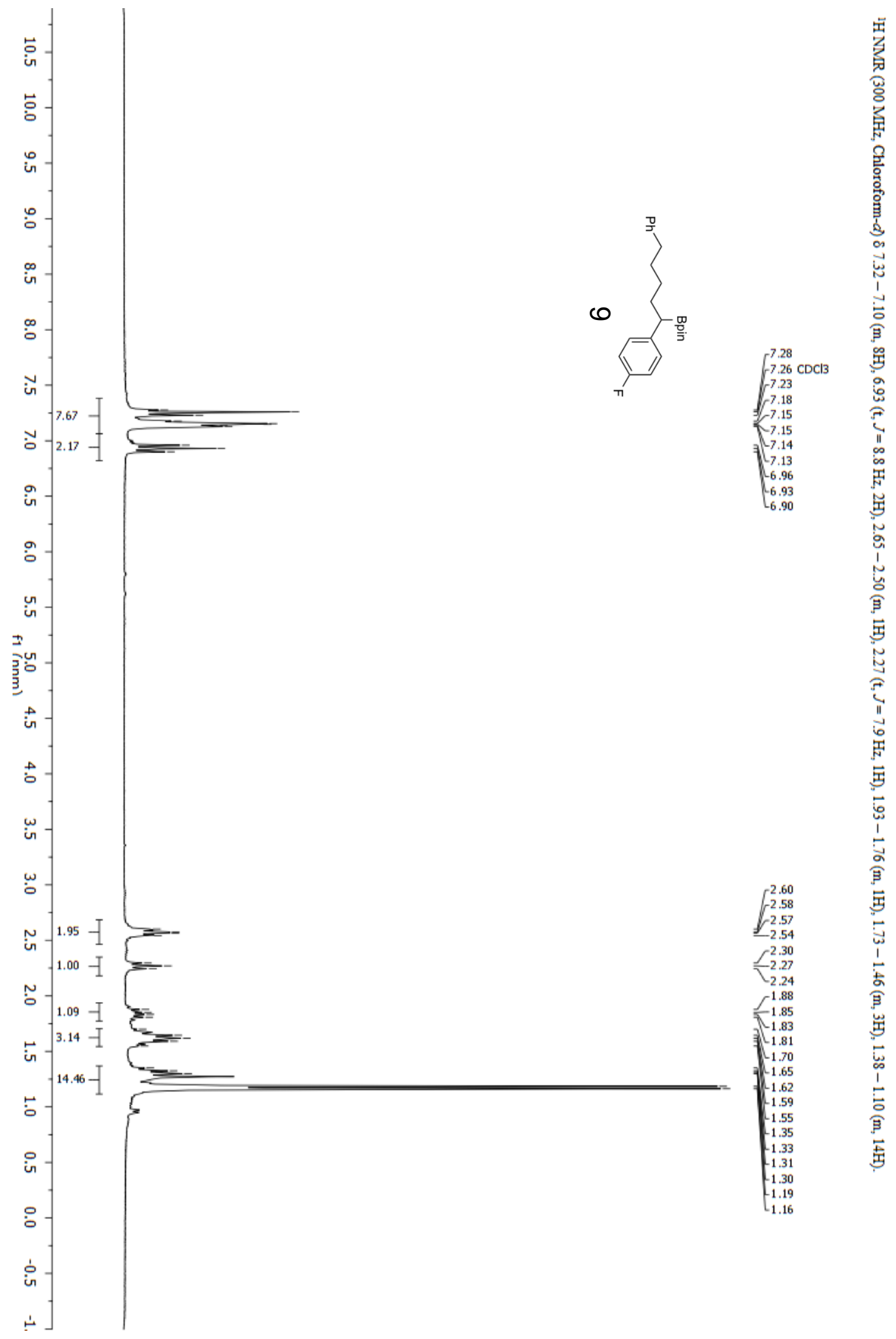




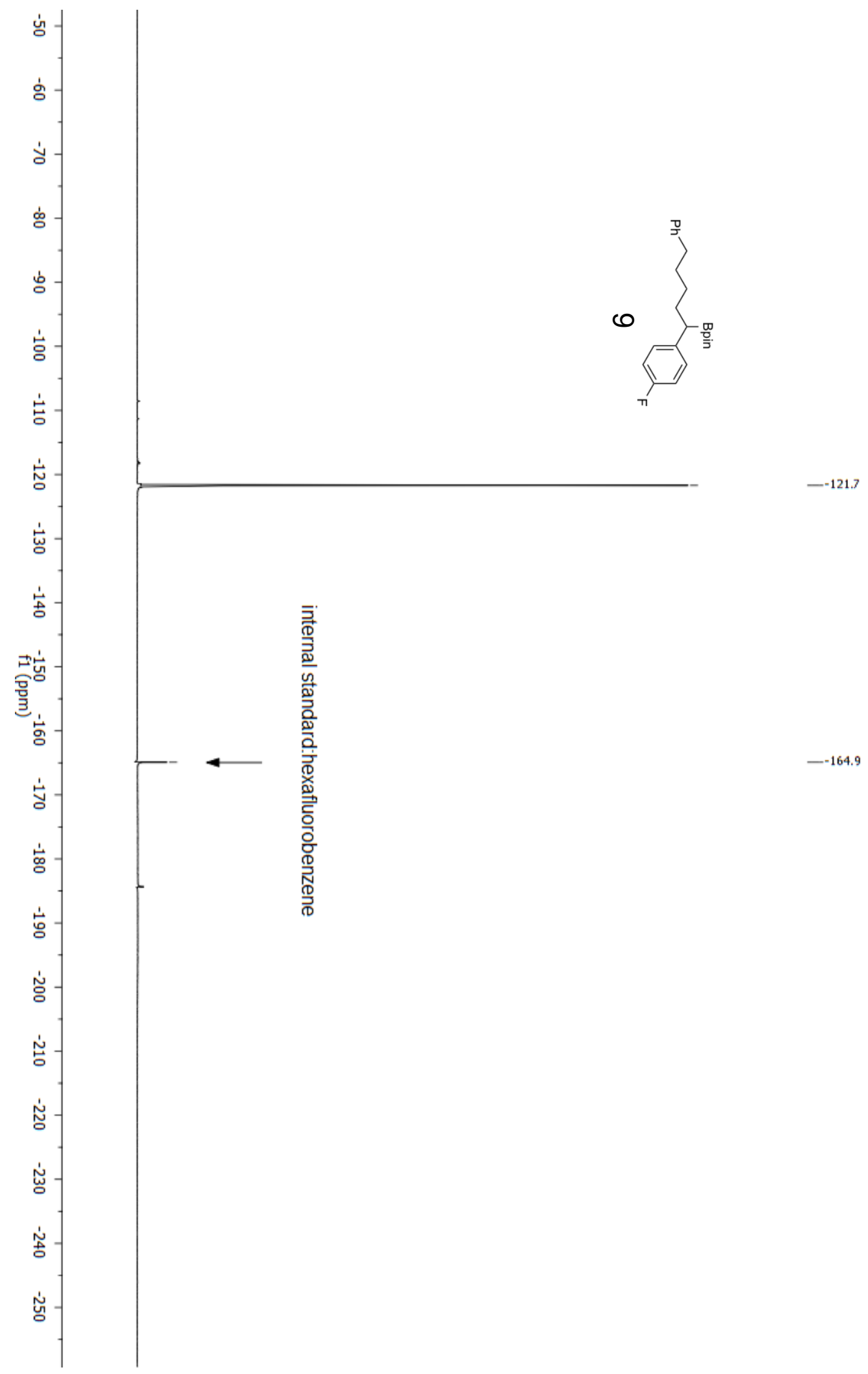



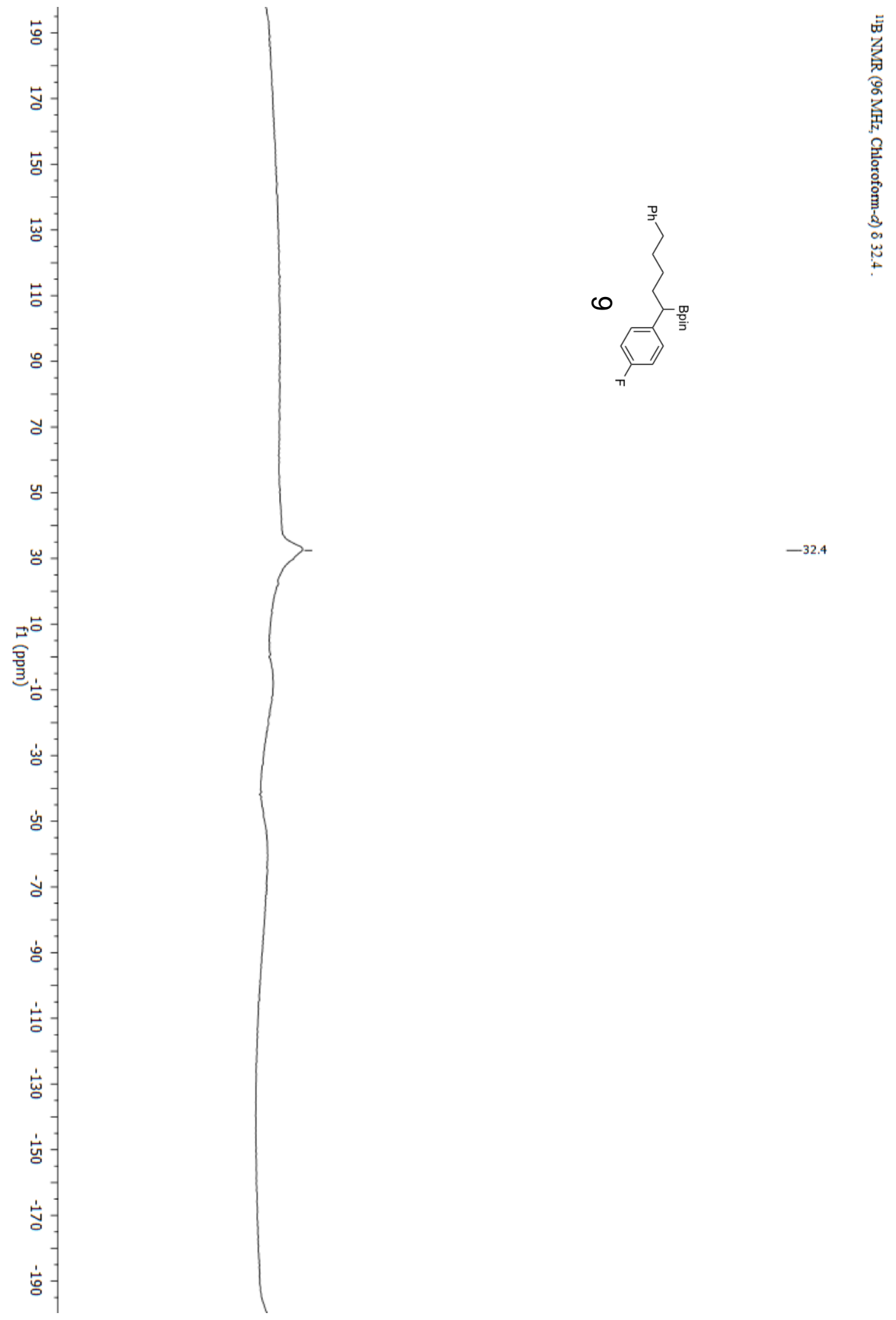

$-32.4$ 


$$
\mid
$$




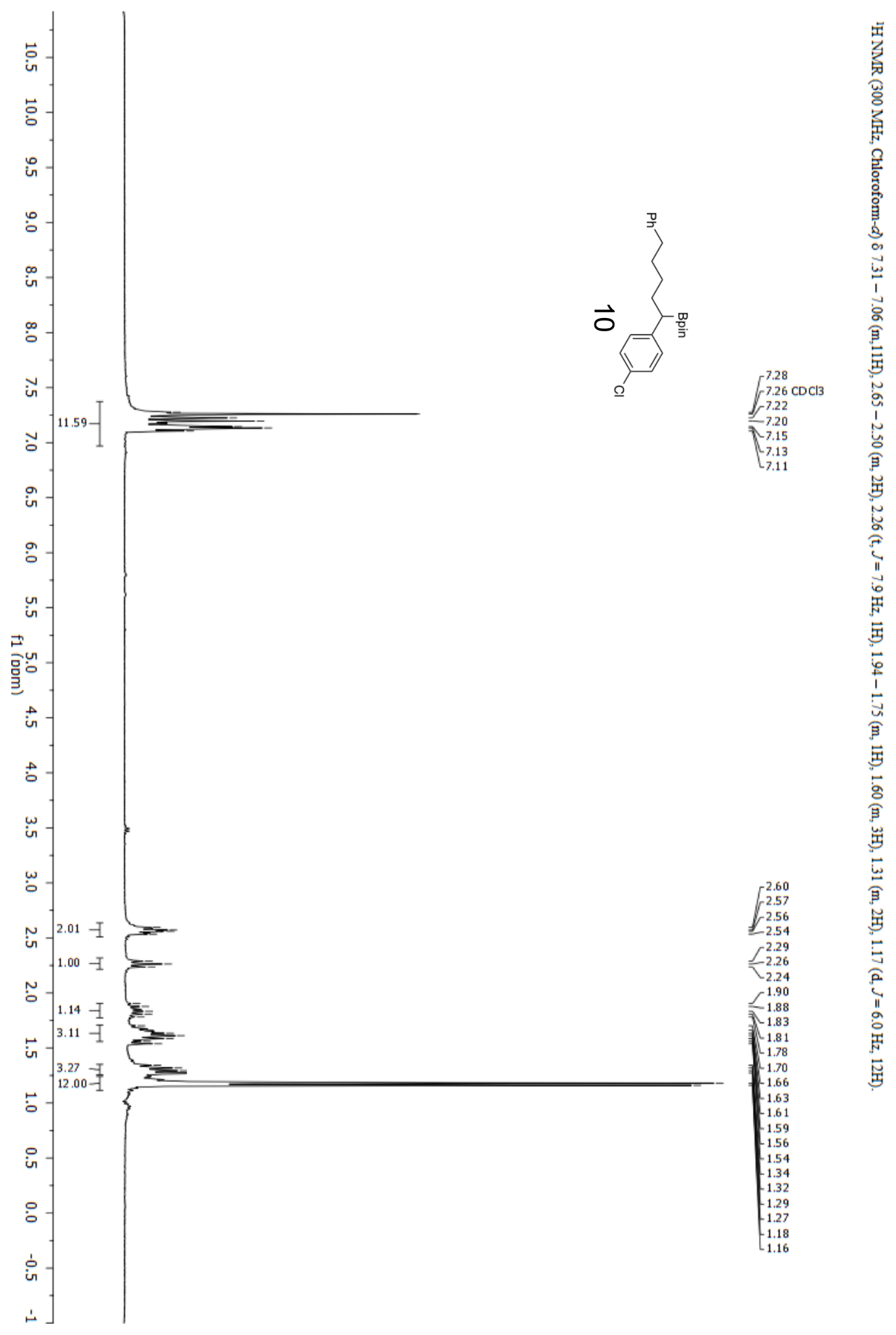



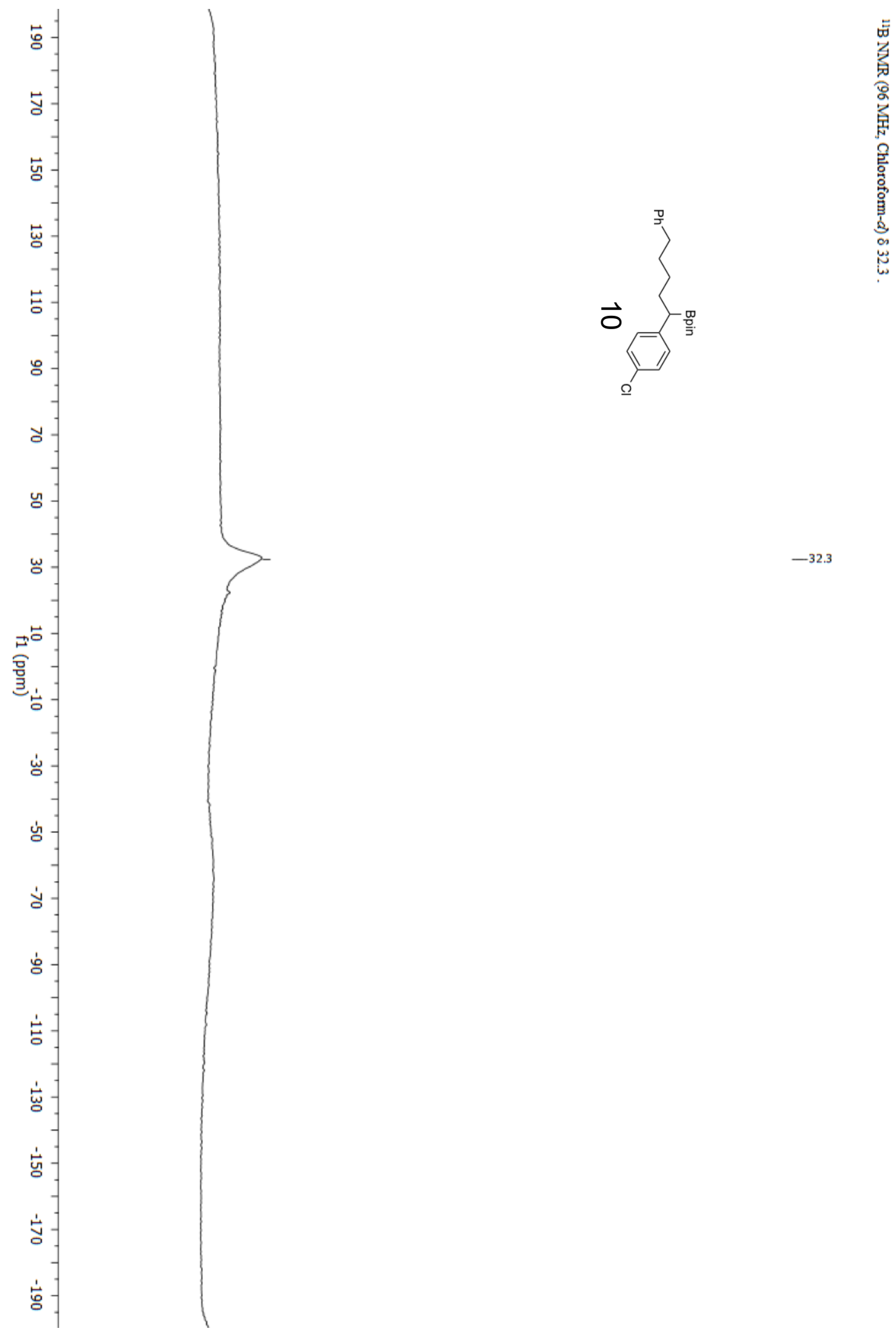

$-32.3$ 


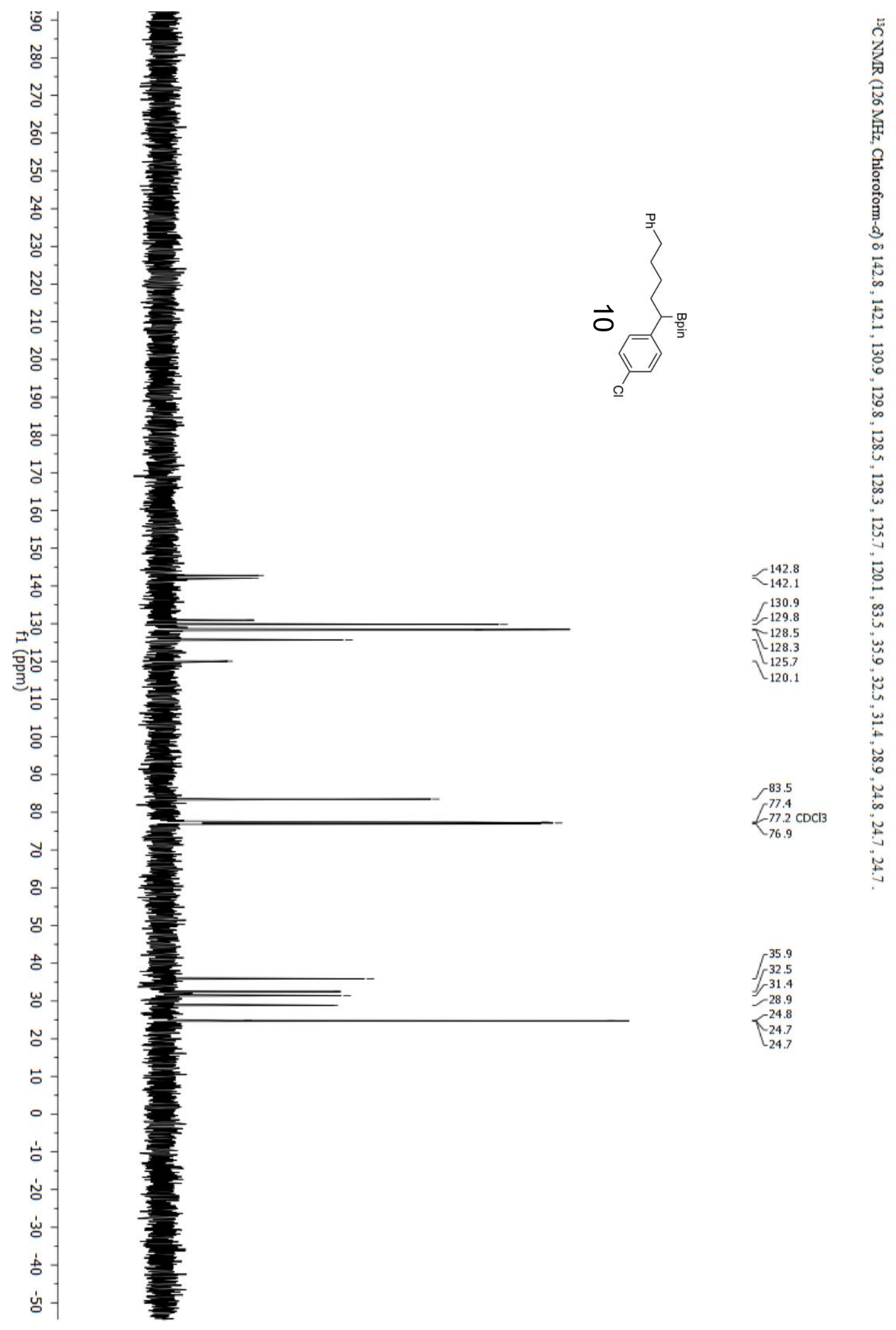




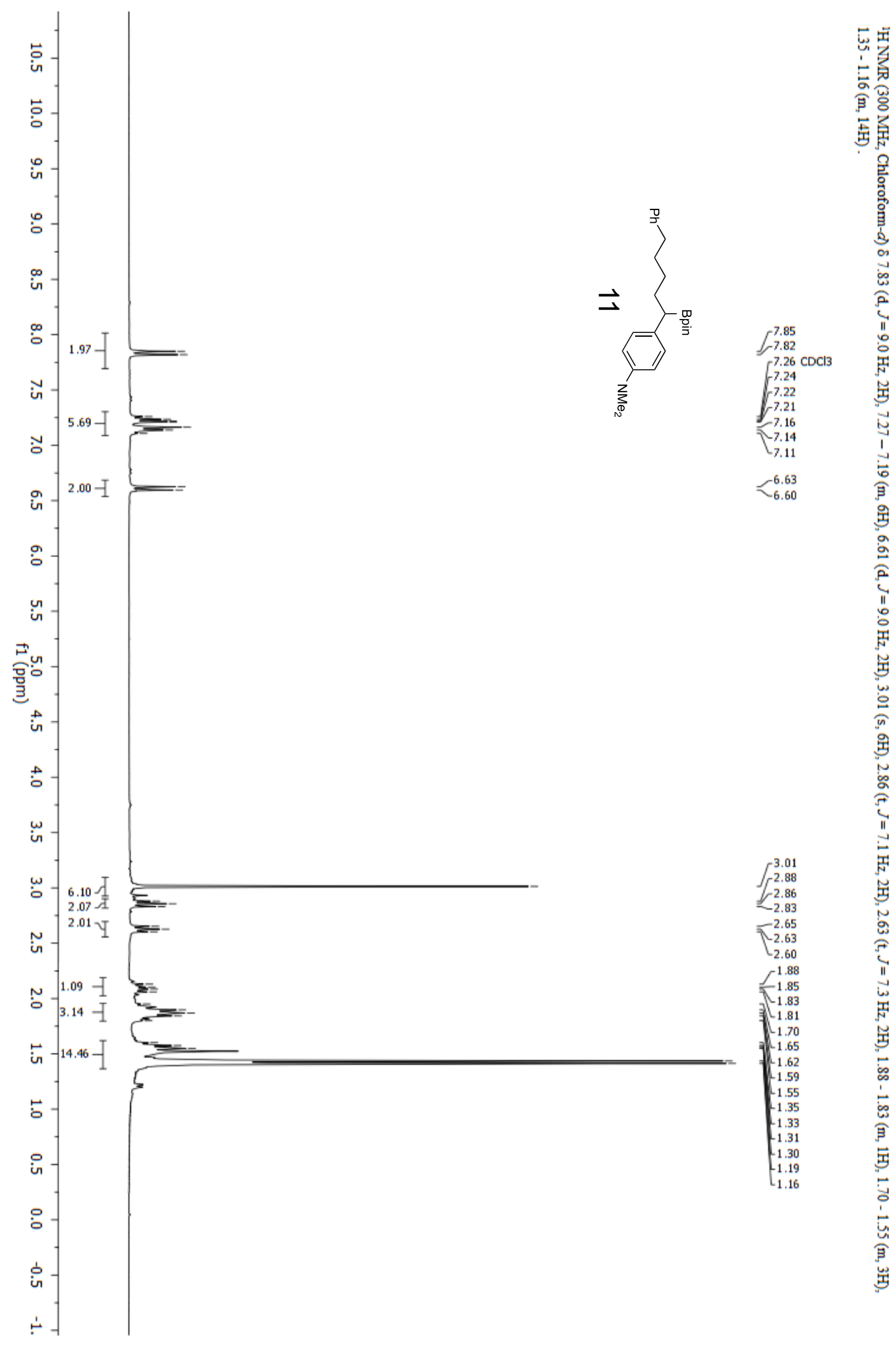



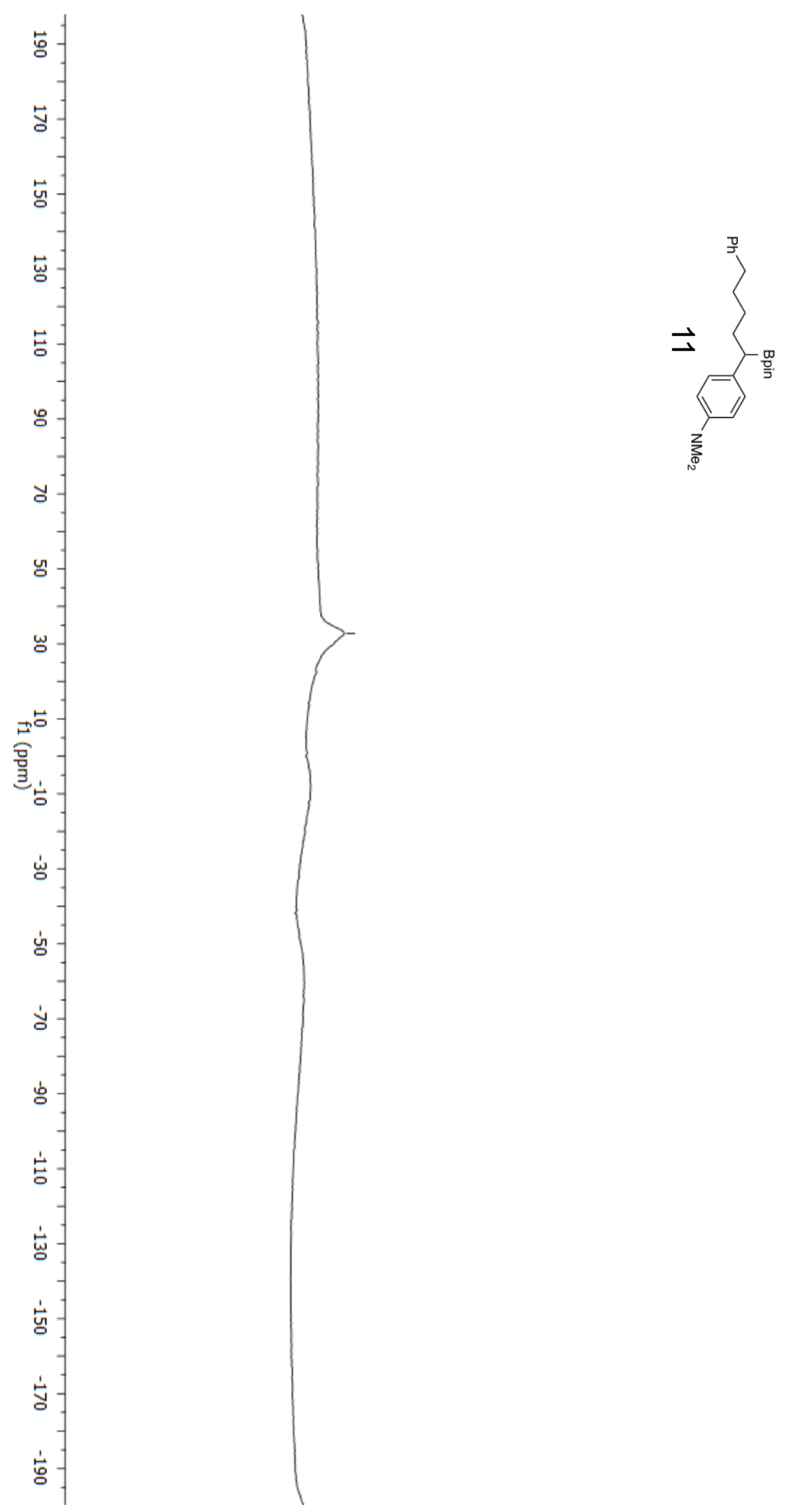

$-32.8$

S44 


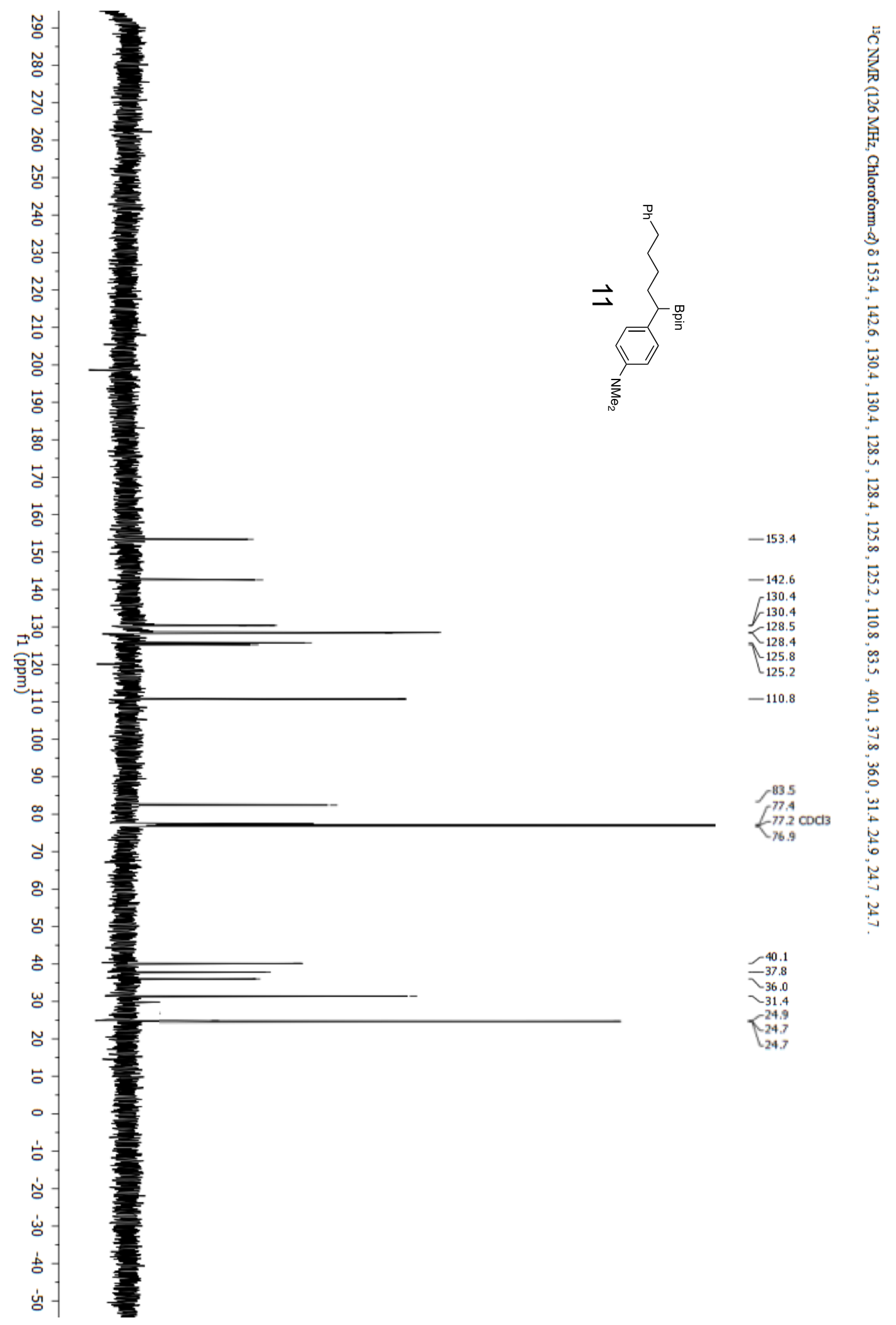




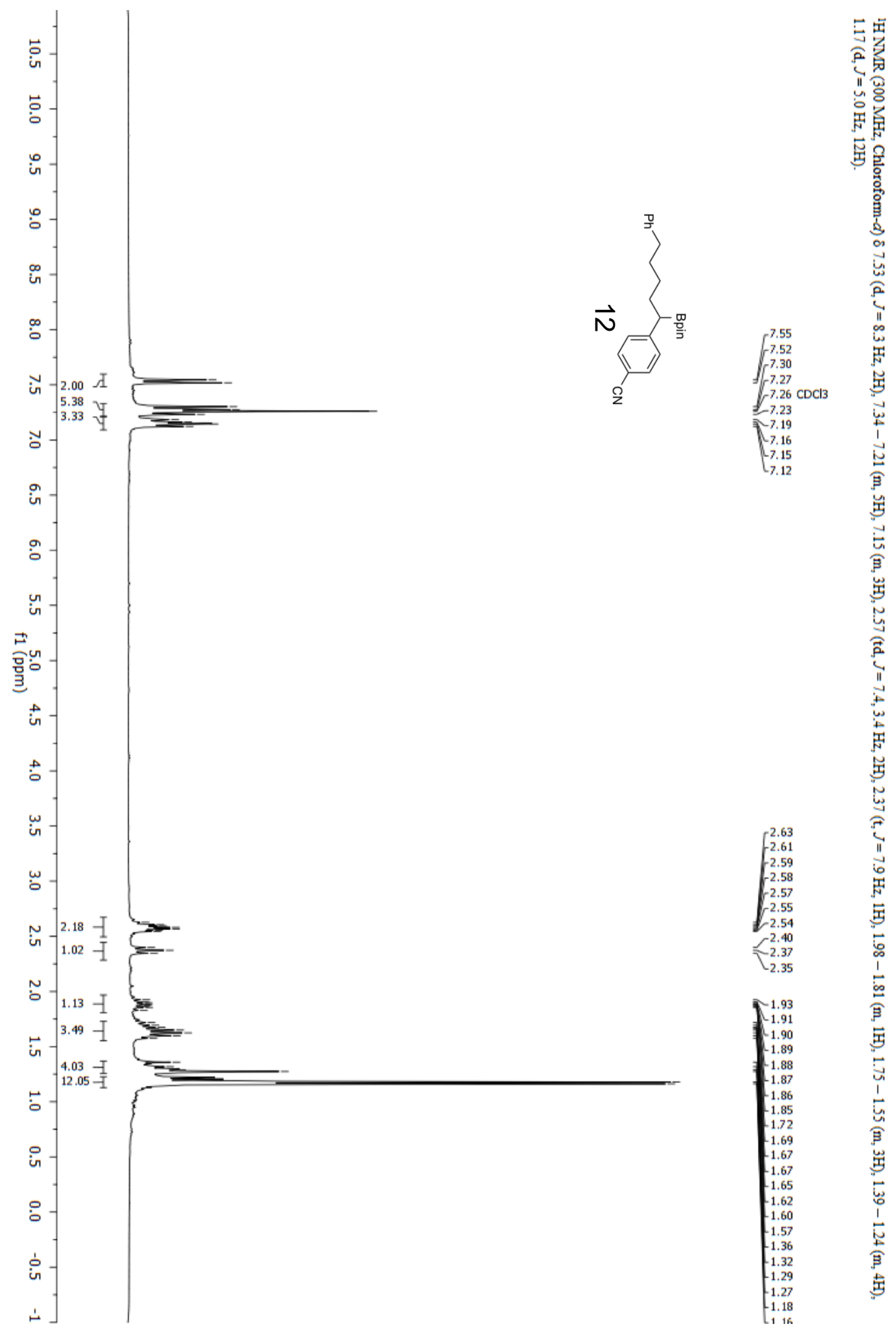



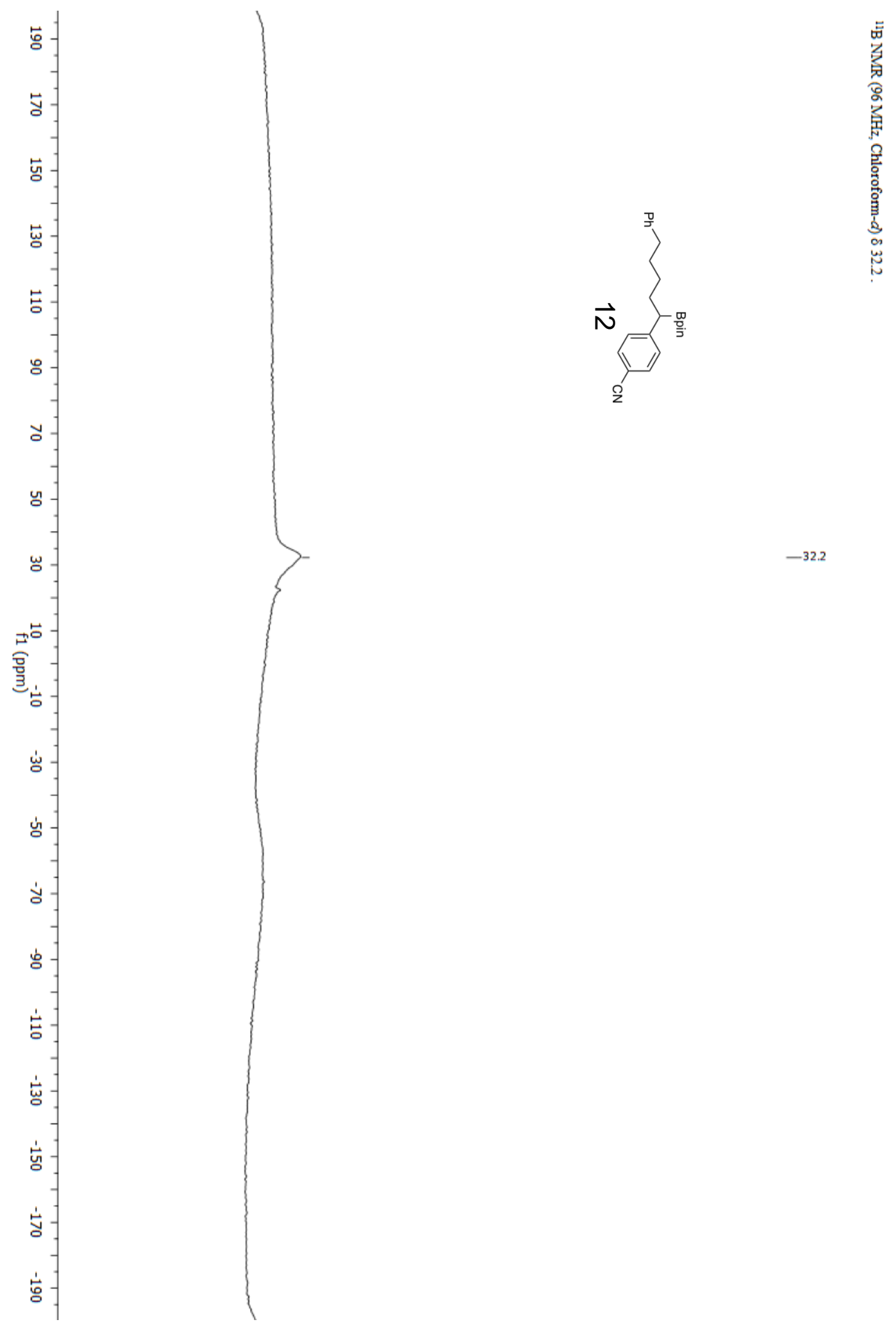


$$
1
$$




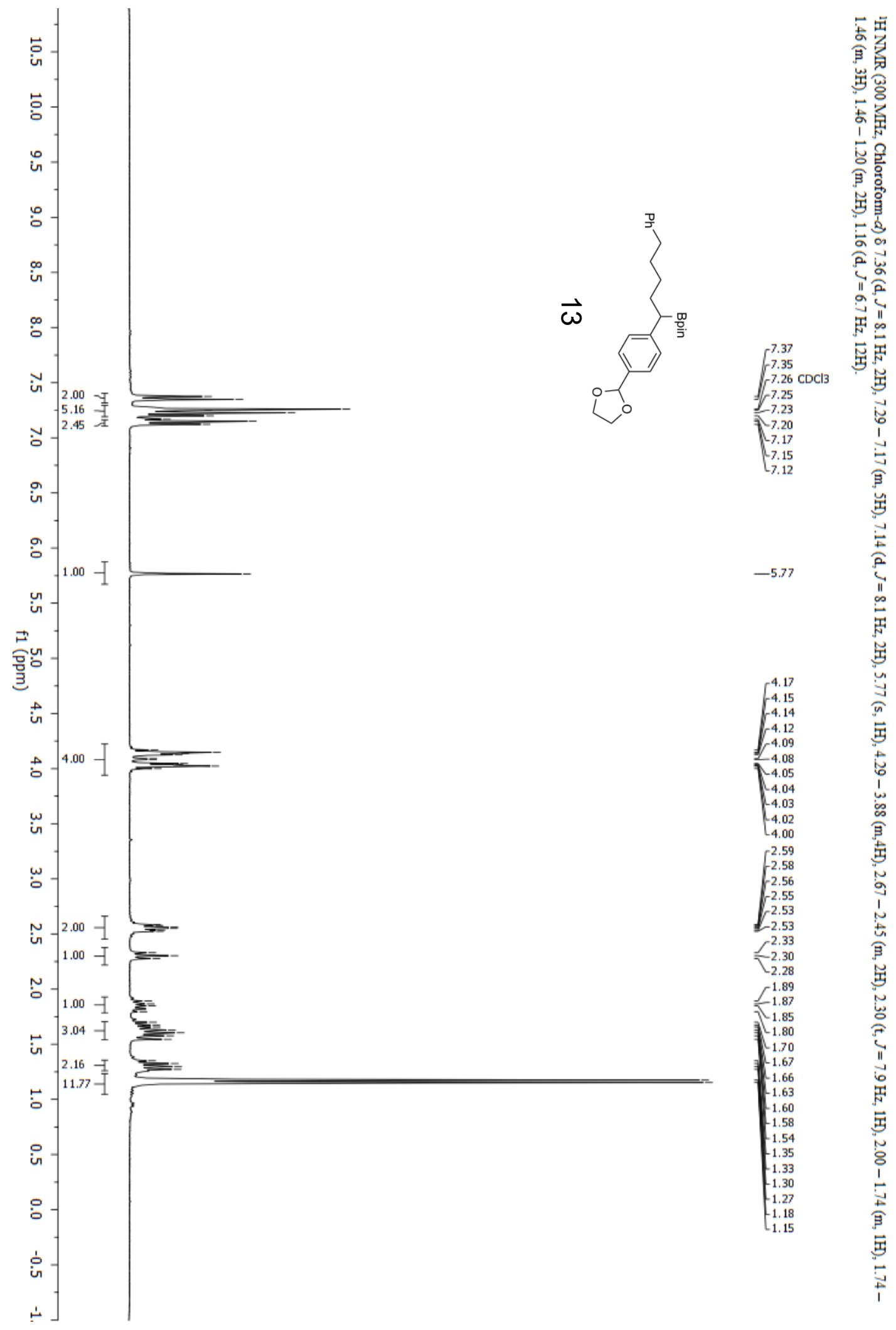



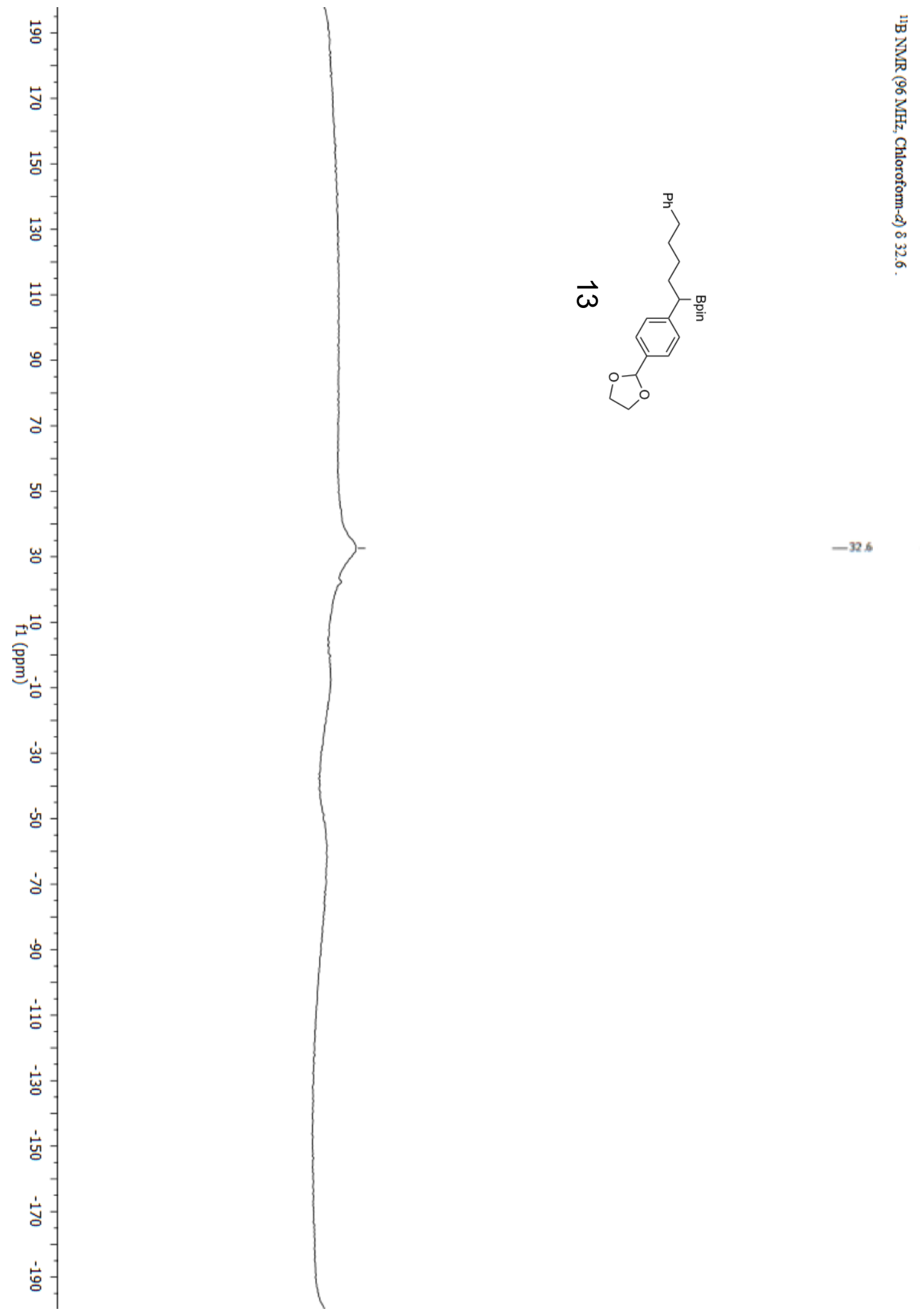

$-32.6$ 


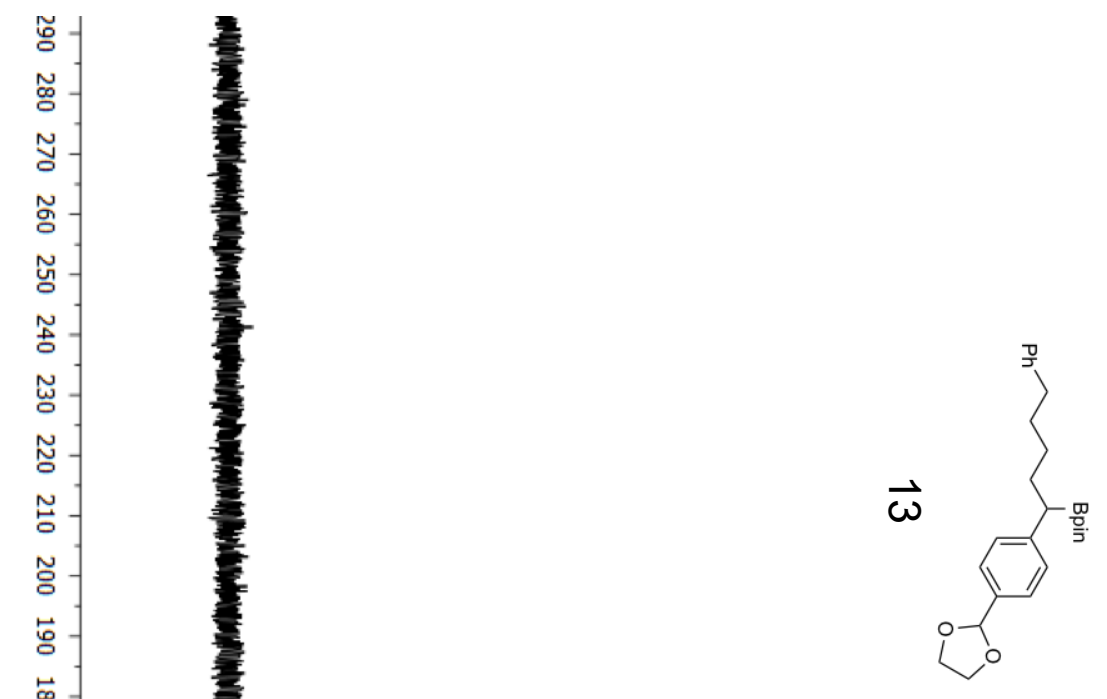

势

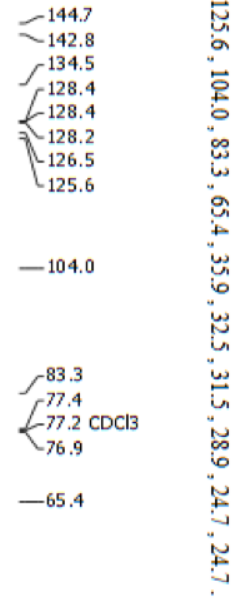

$$
\begin{array}{r}
35.9 \\
-32.5 \\
-31.5 \\
-28.9 \\
\square-24.7 \\
24.7
\end{array}
$$

S51 


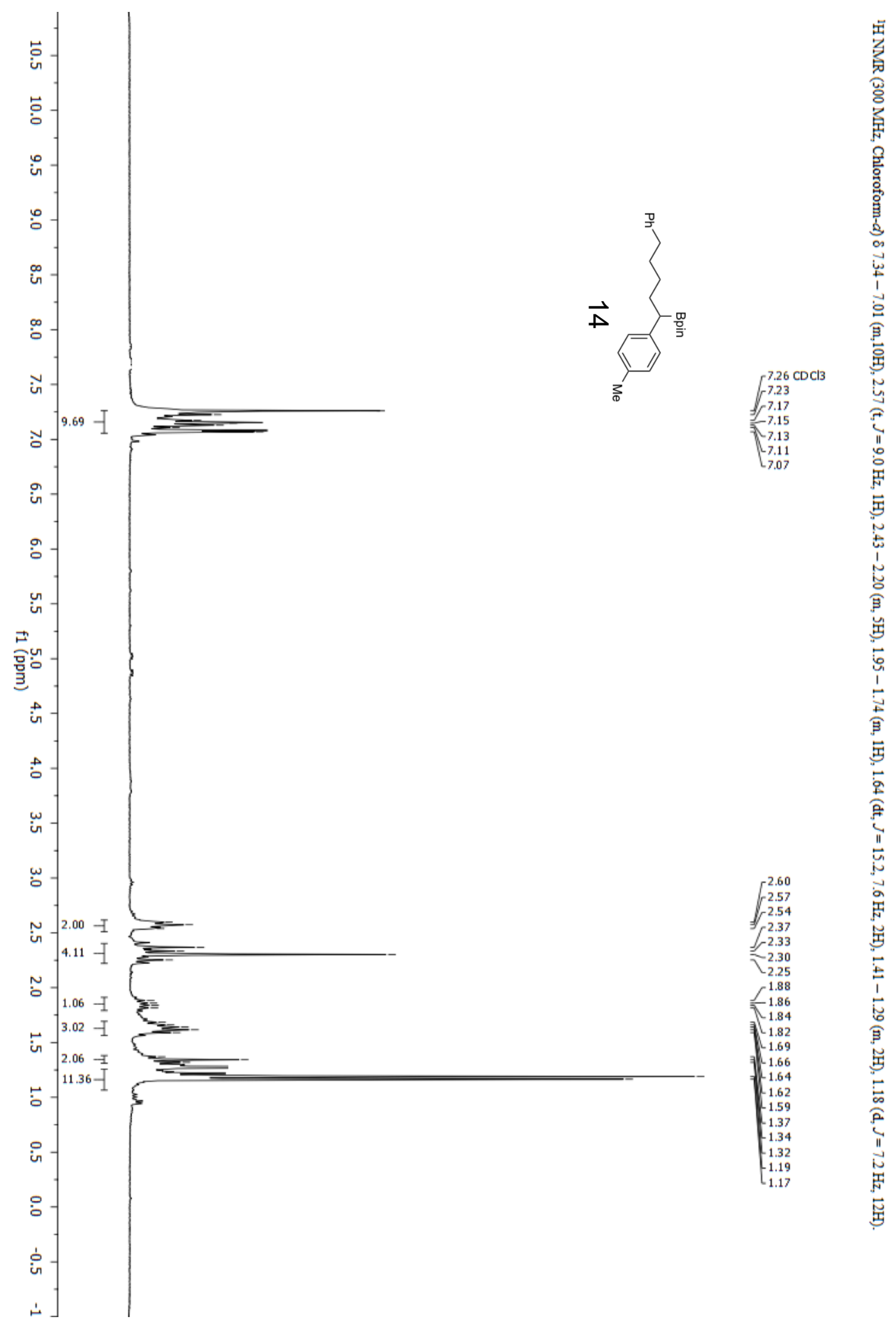



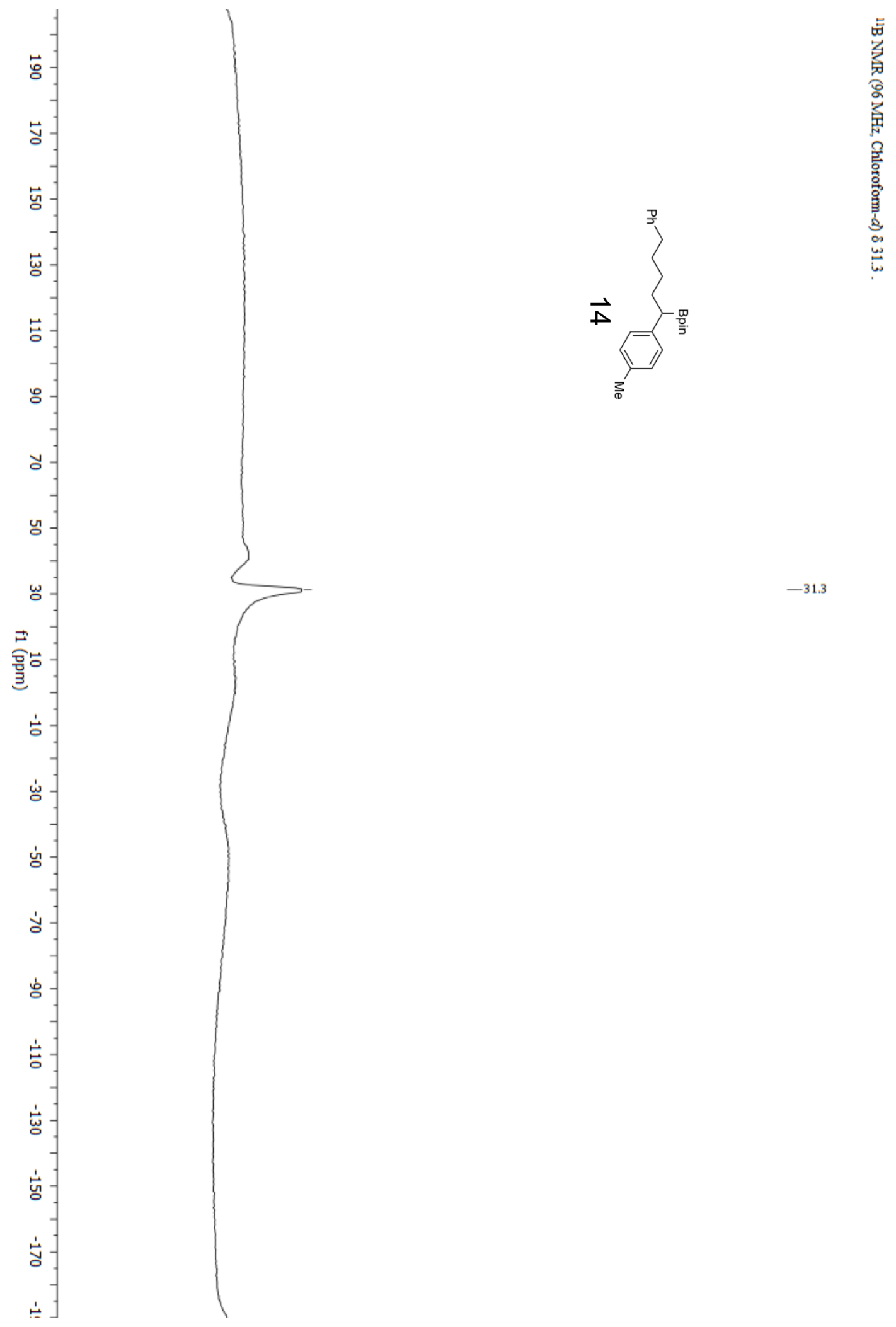


$$
\mid
$$




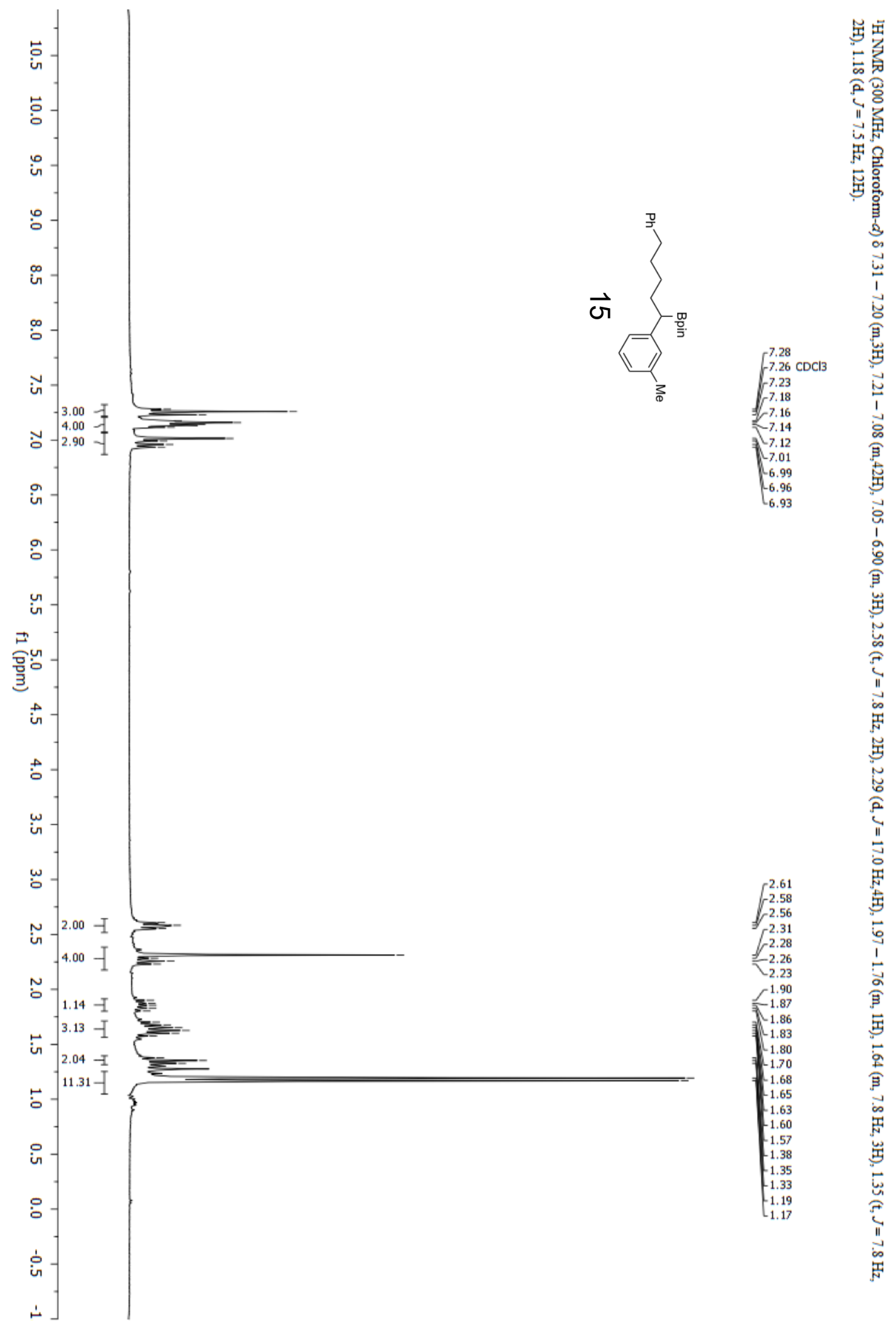




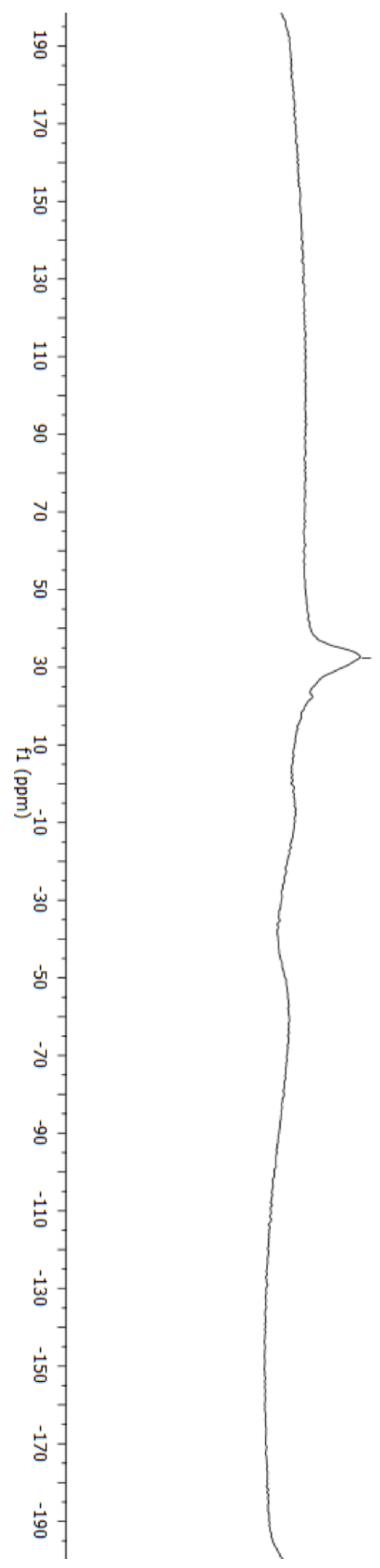




$$
\mid
$$




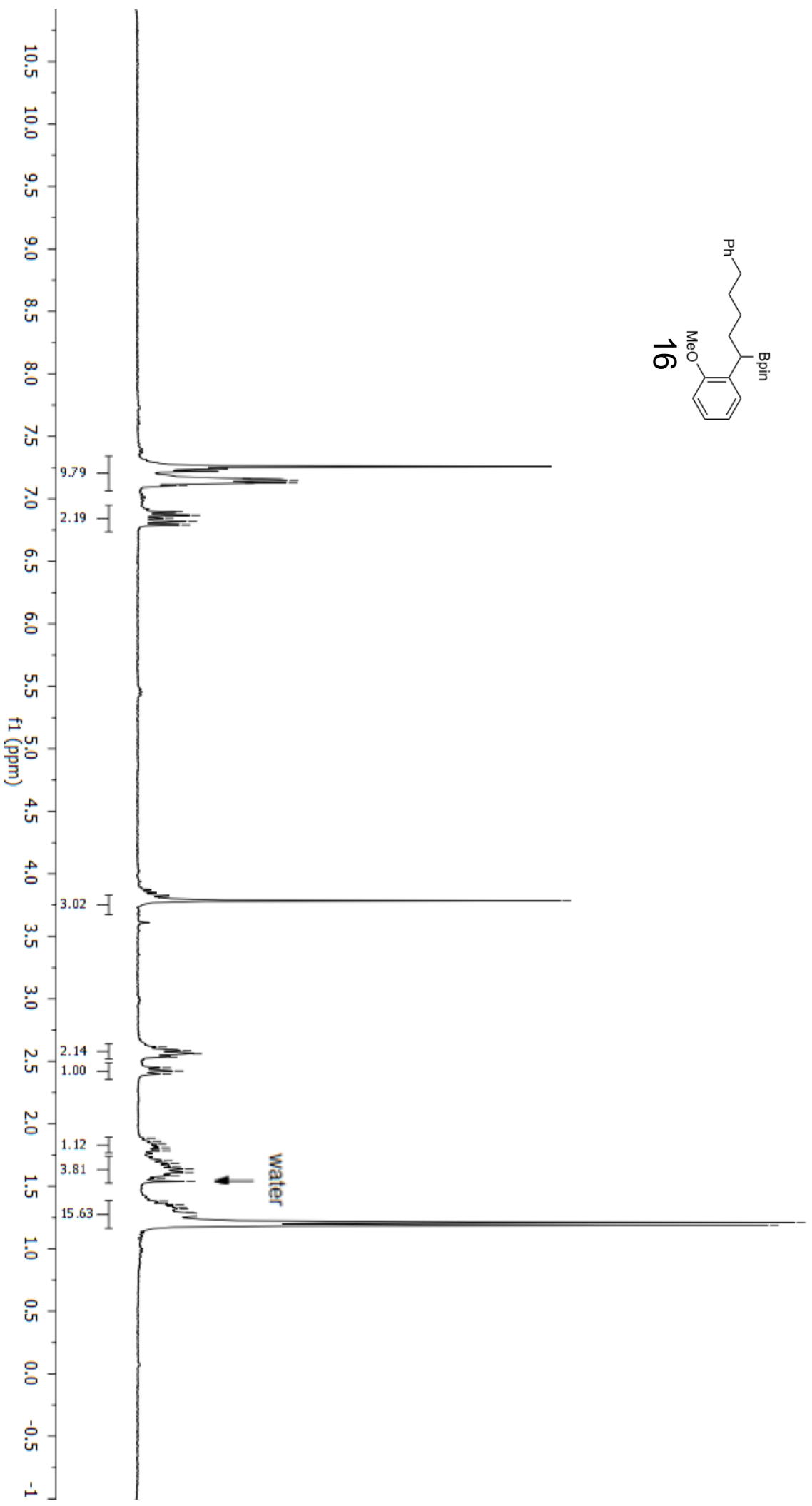

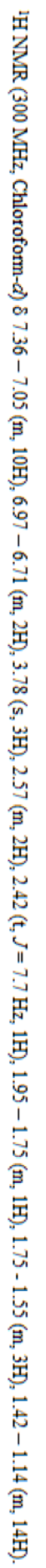

S58 

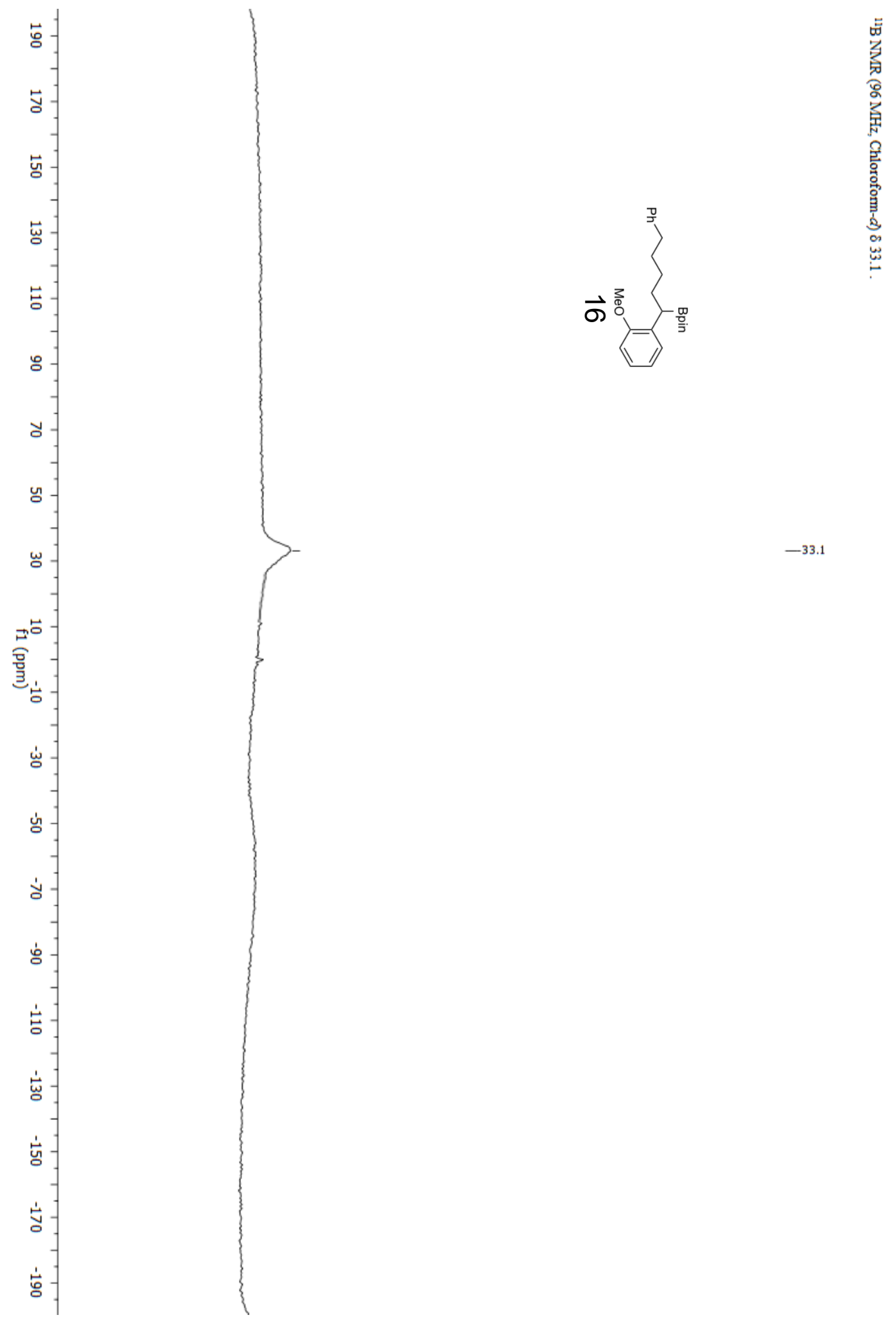


$$
\mid
$$




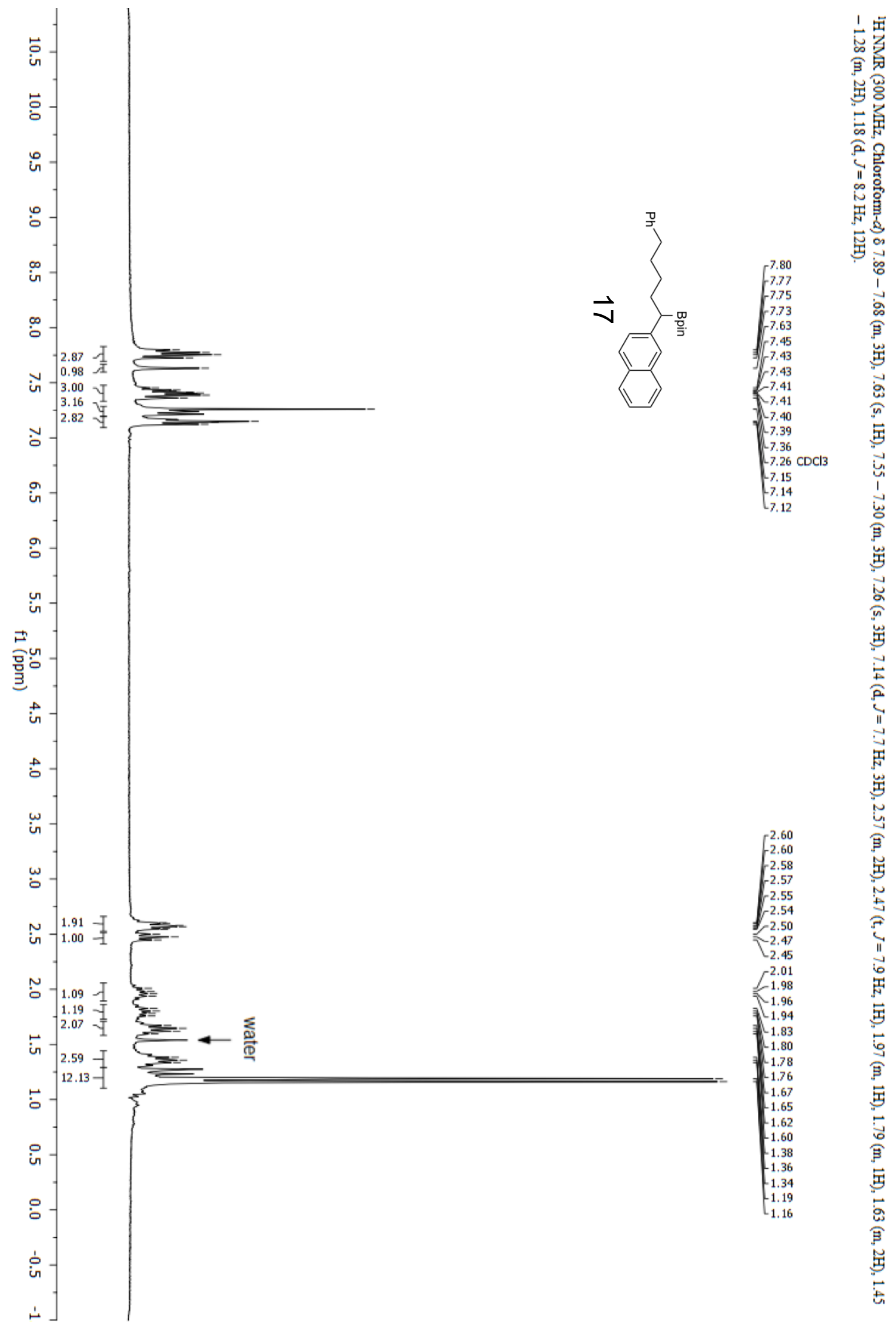



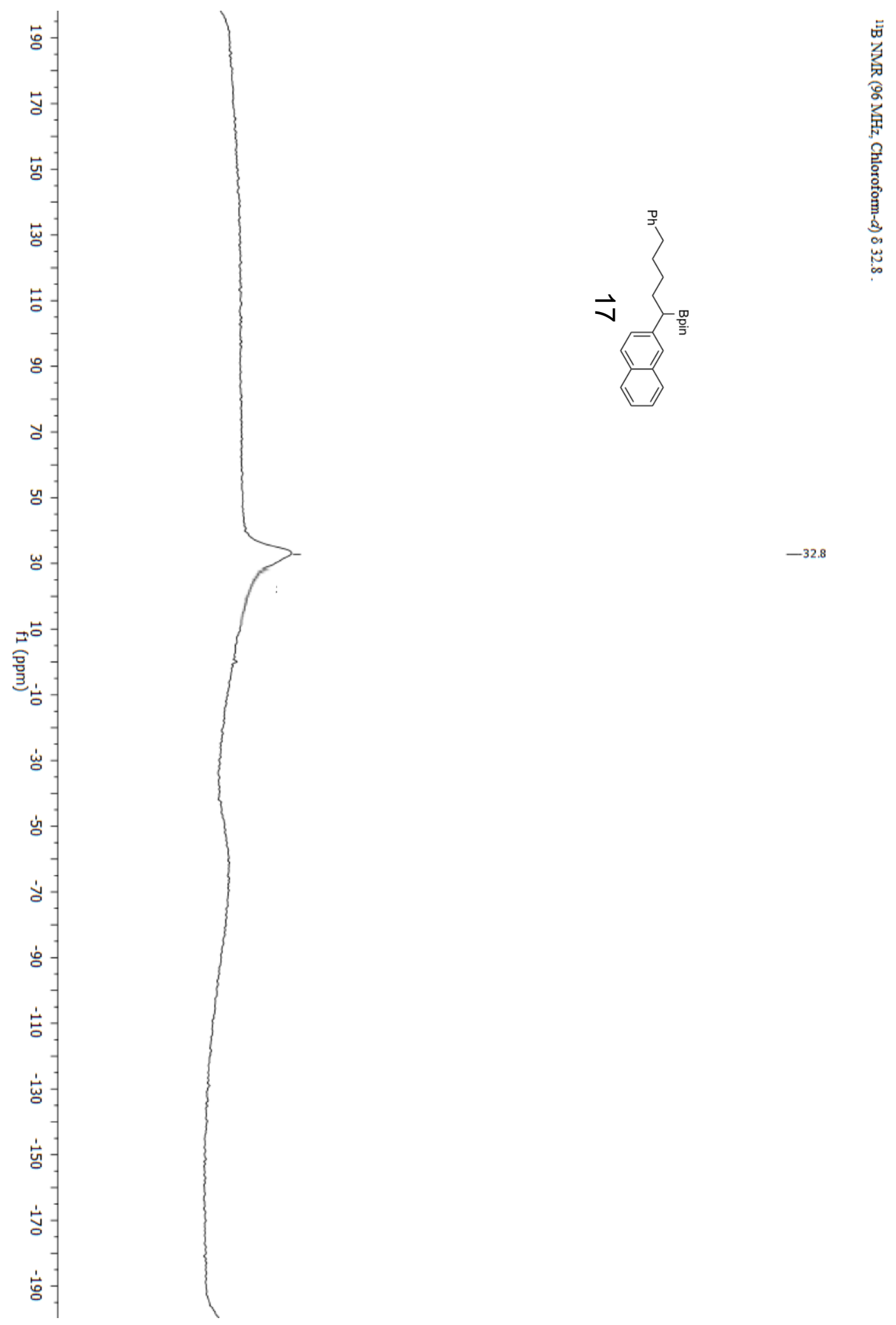

$-32.8$ 


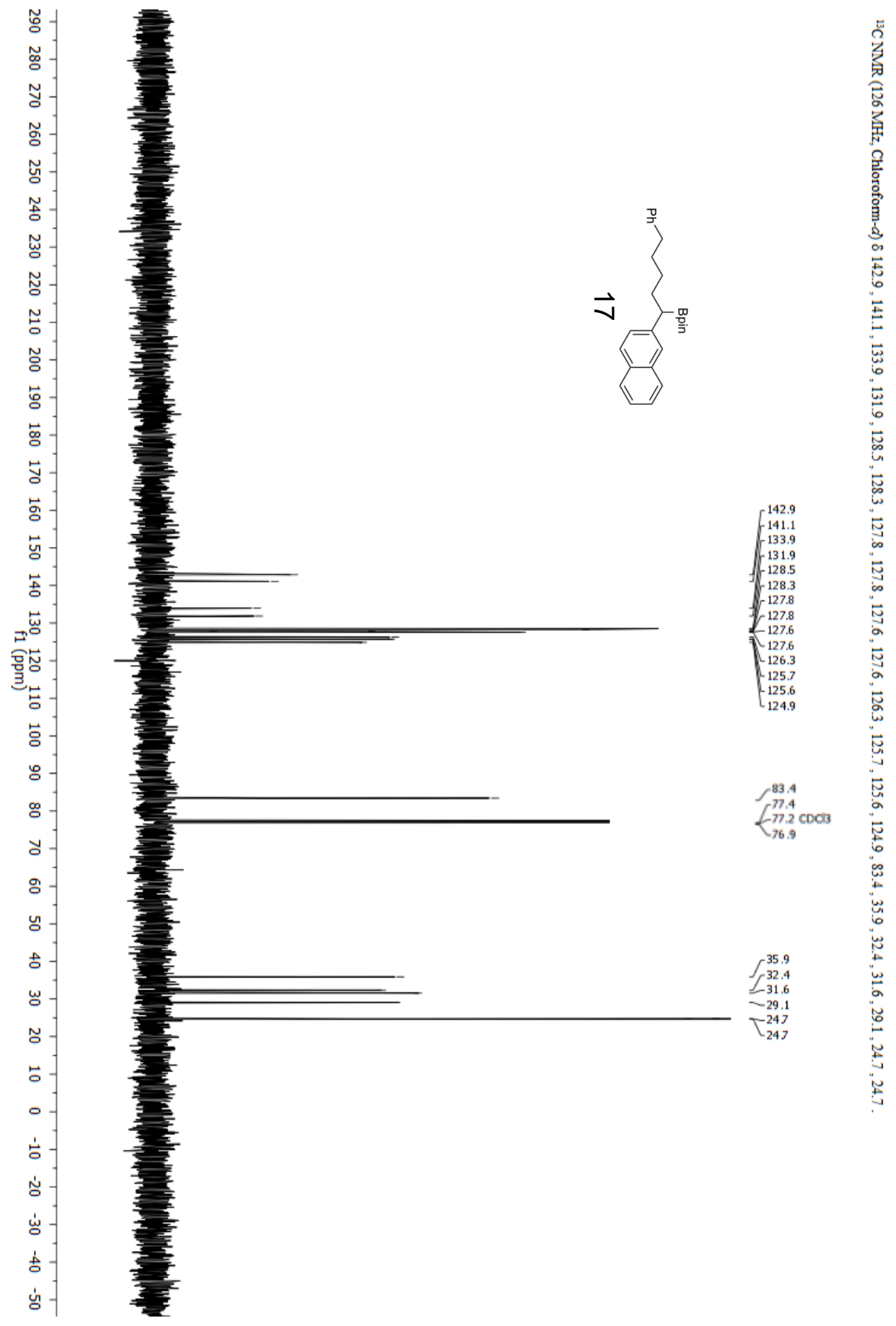




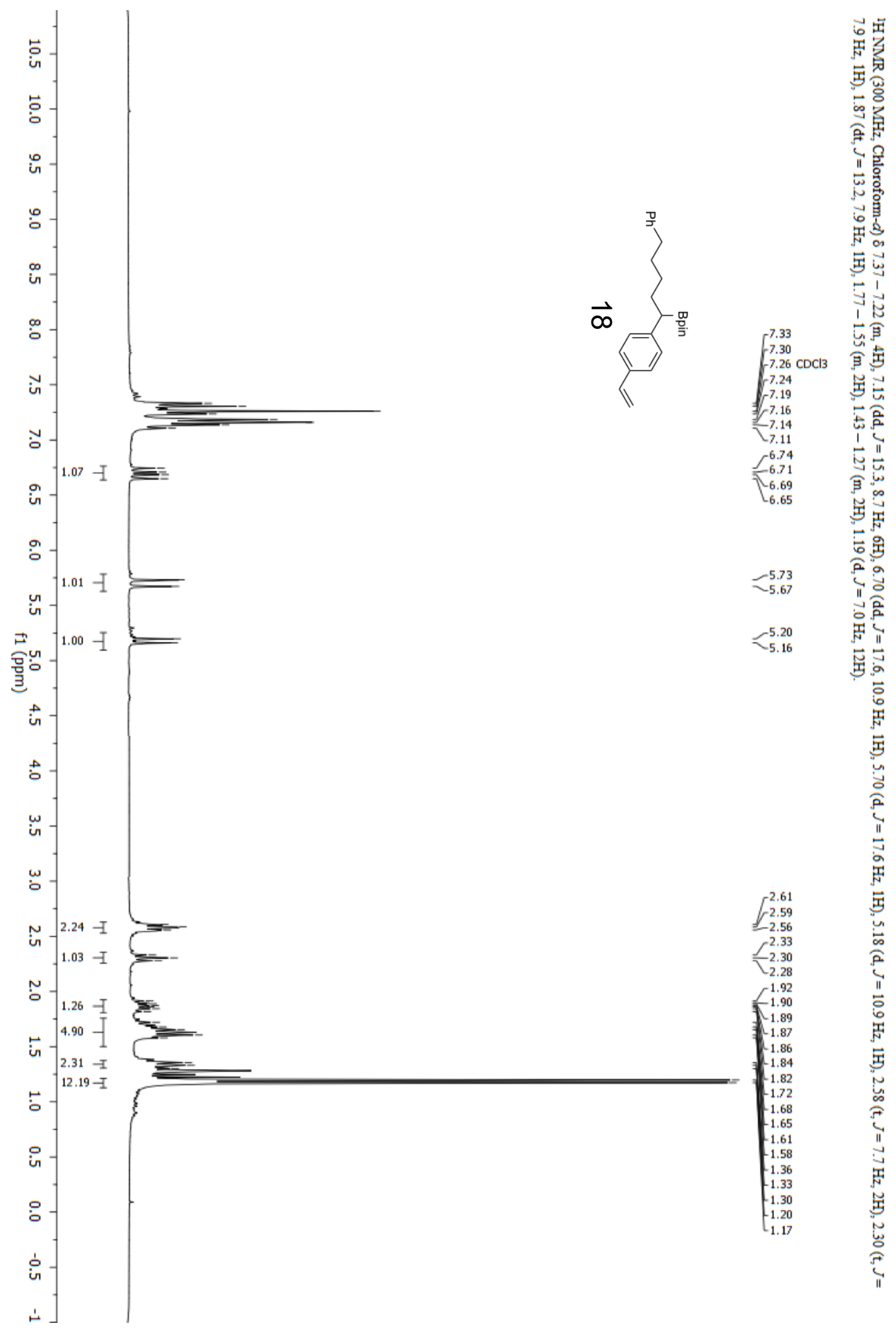



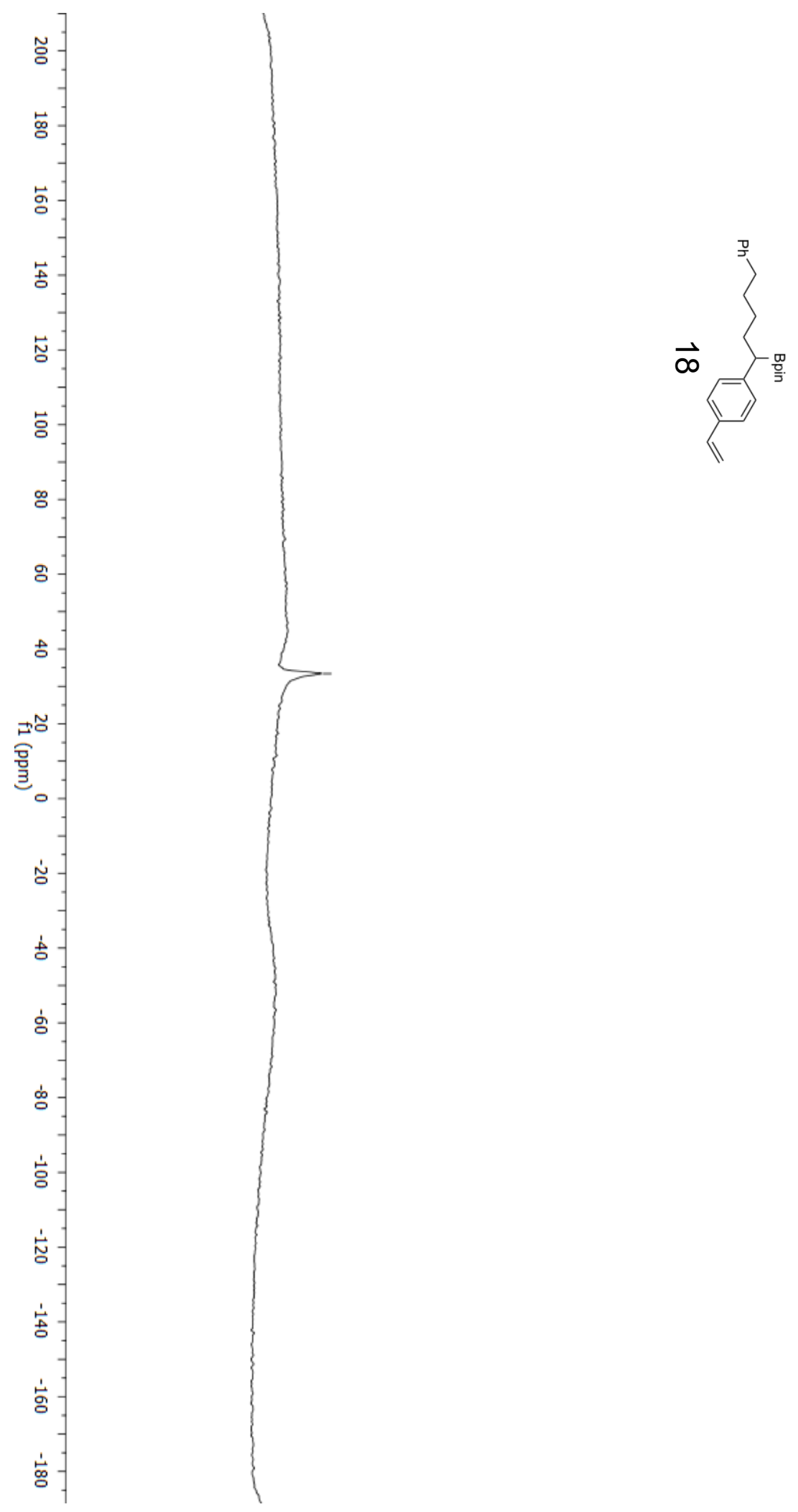

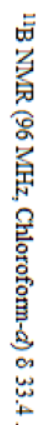

$-33.4$ 


$$
\mid k
$$




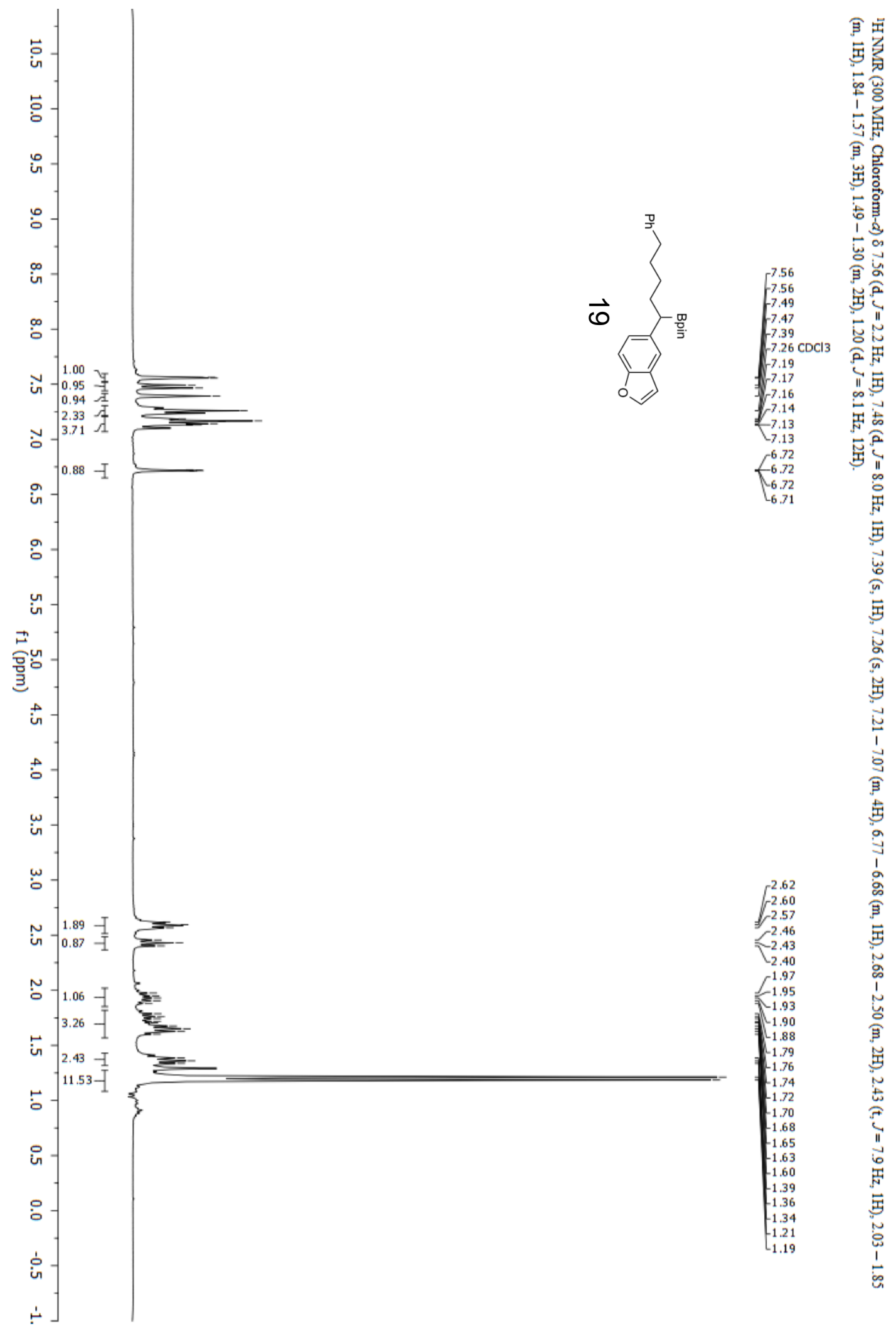




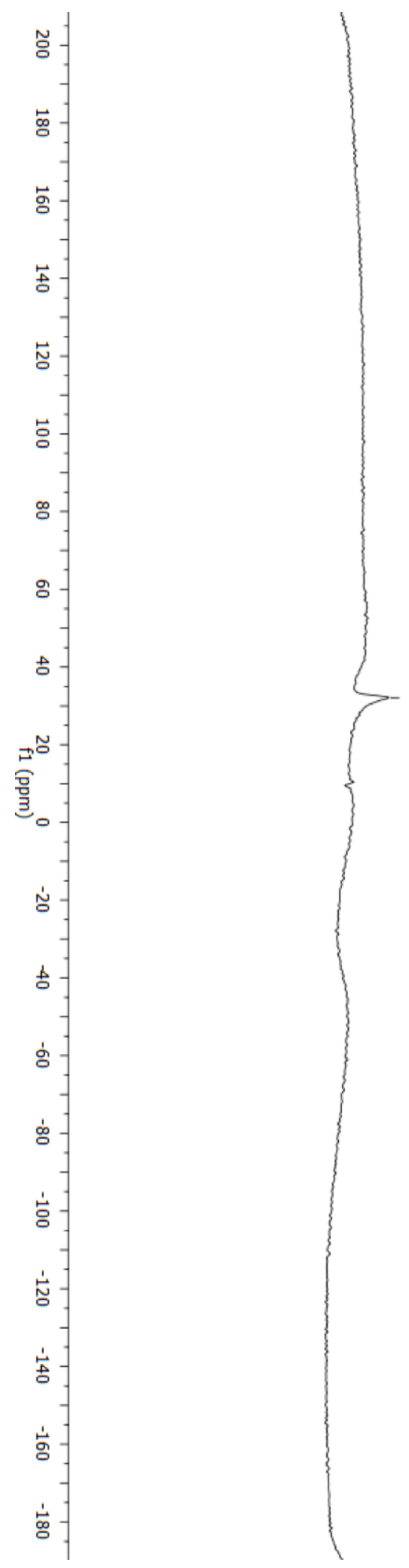




$$
\mid
$$




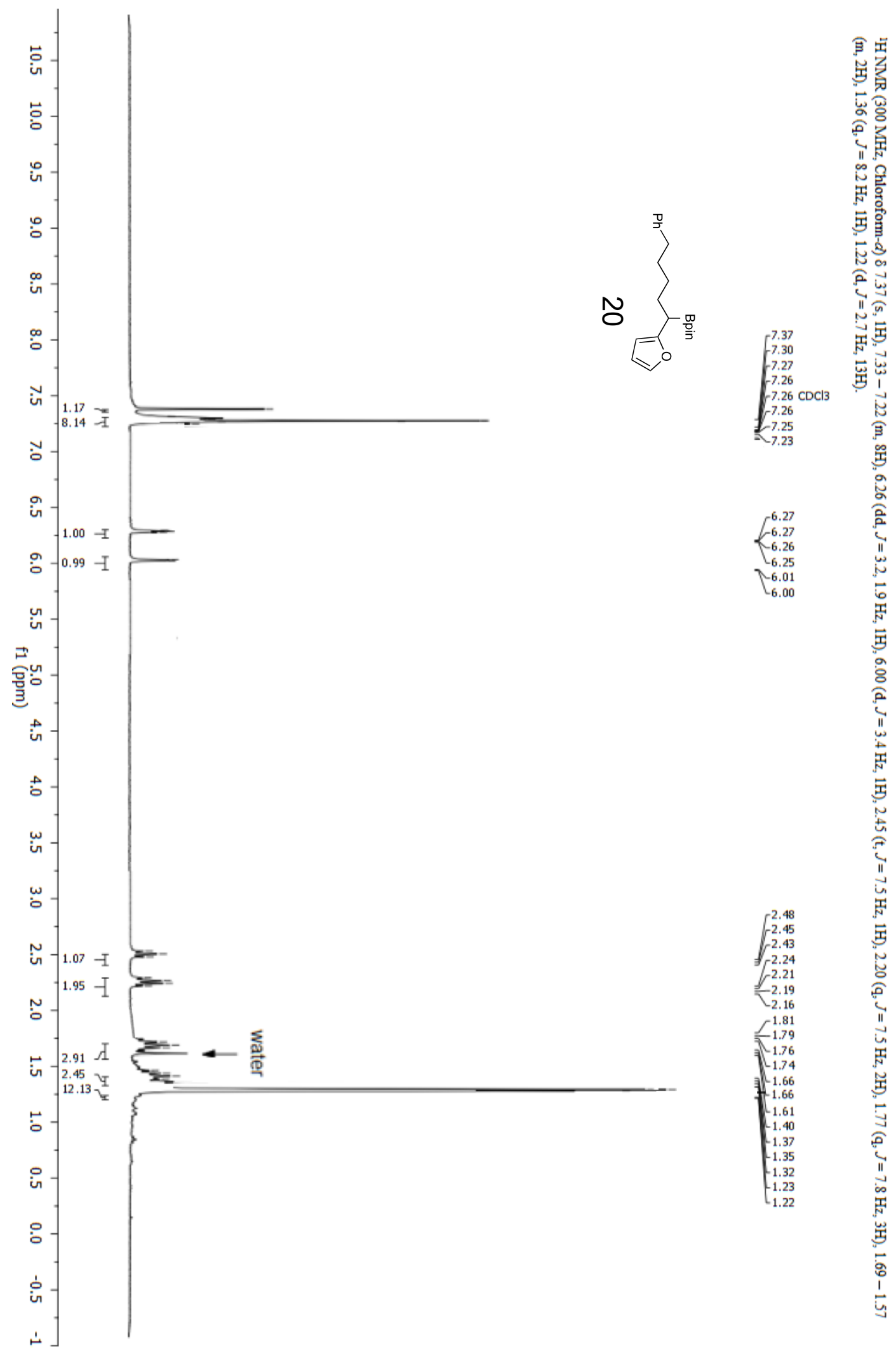



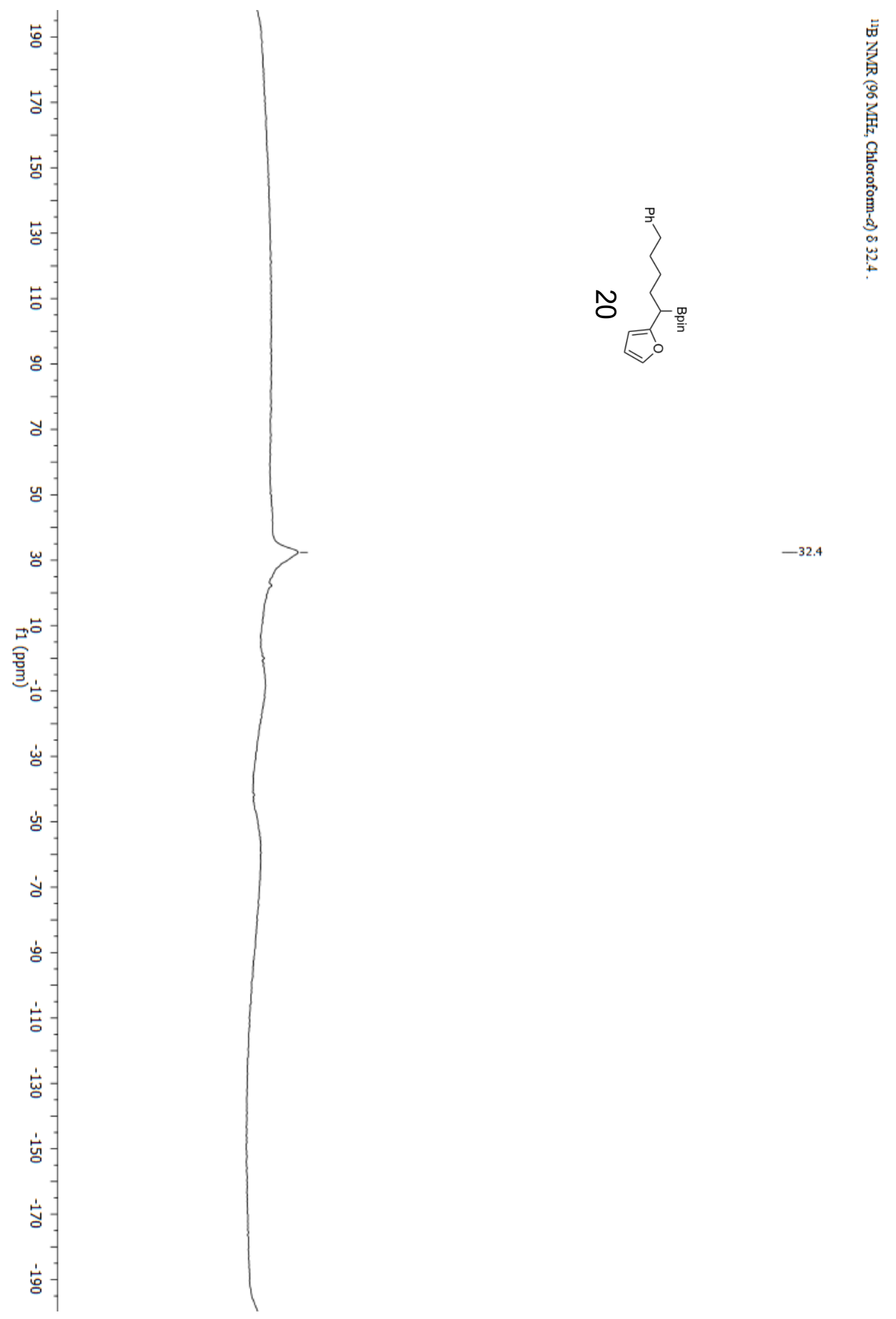

$-32.4$

S71 


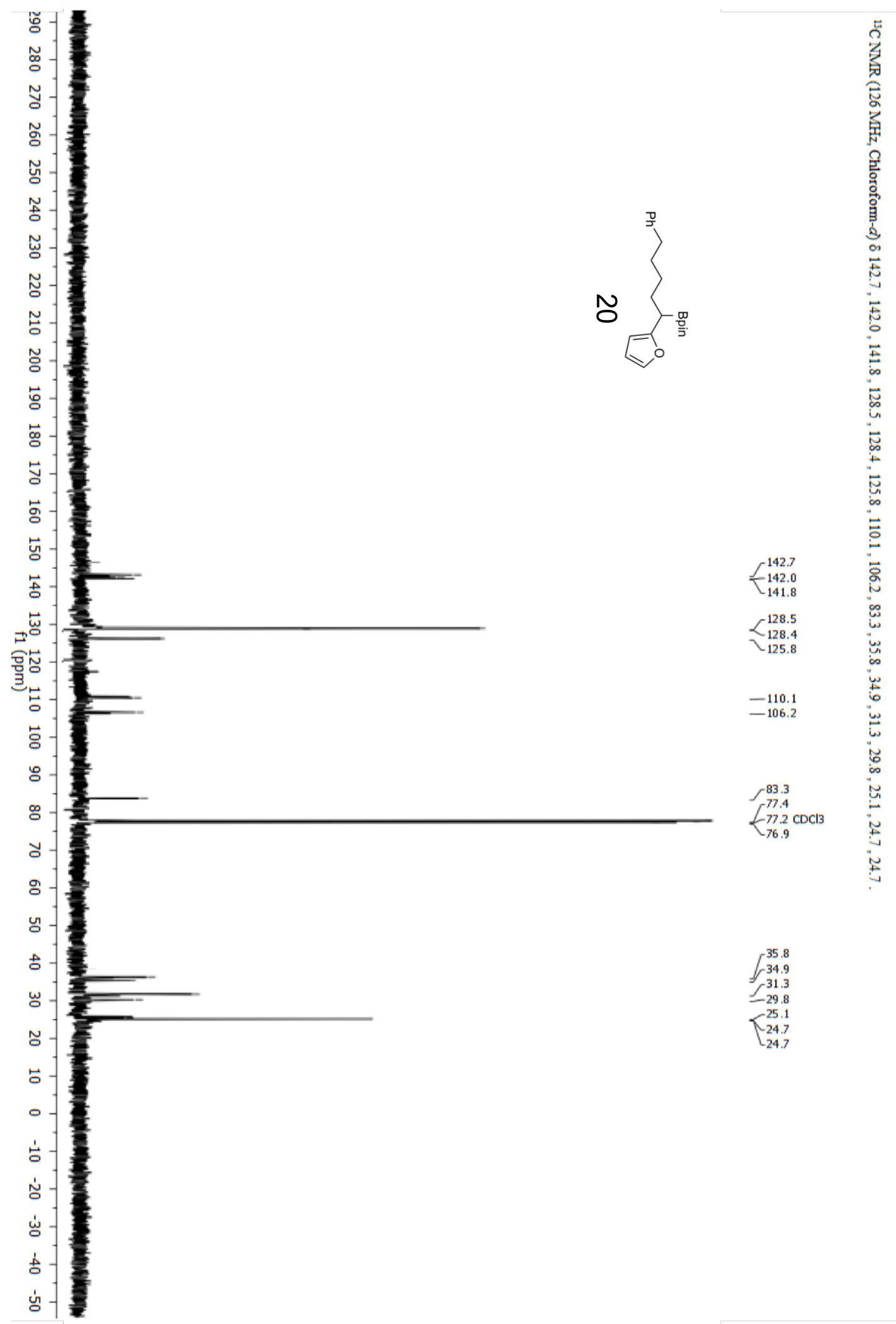




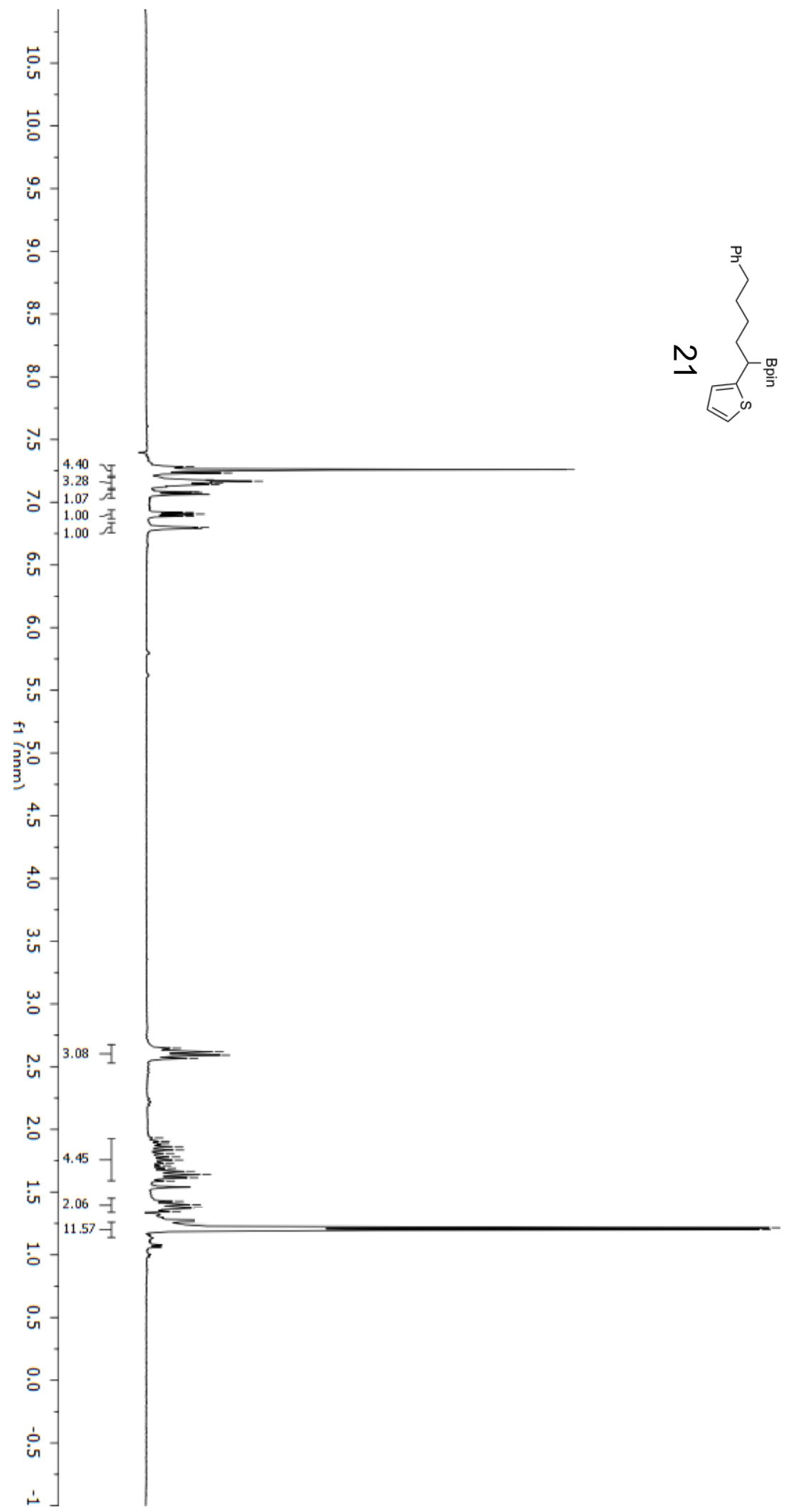



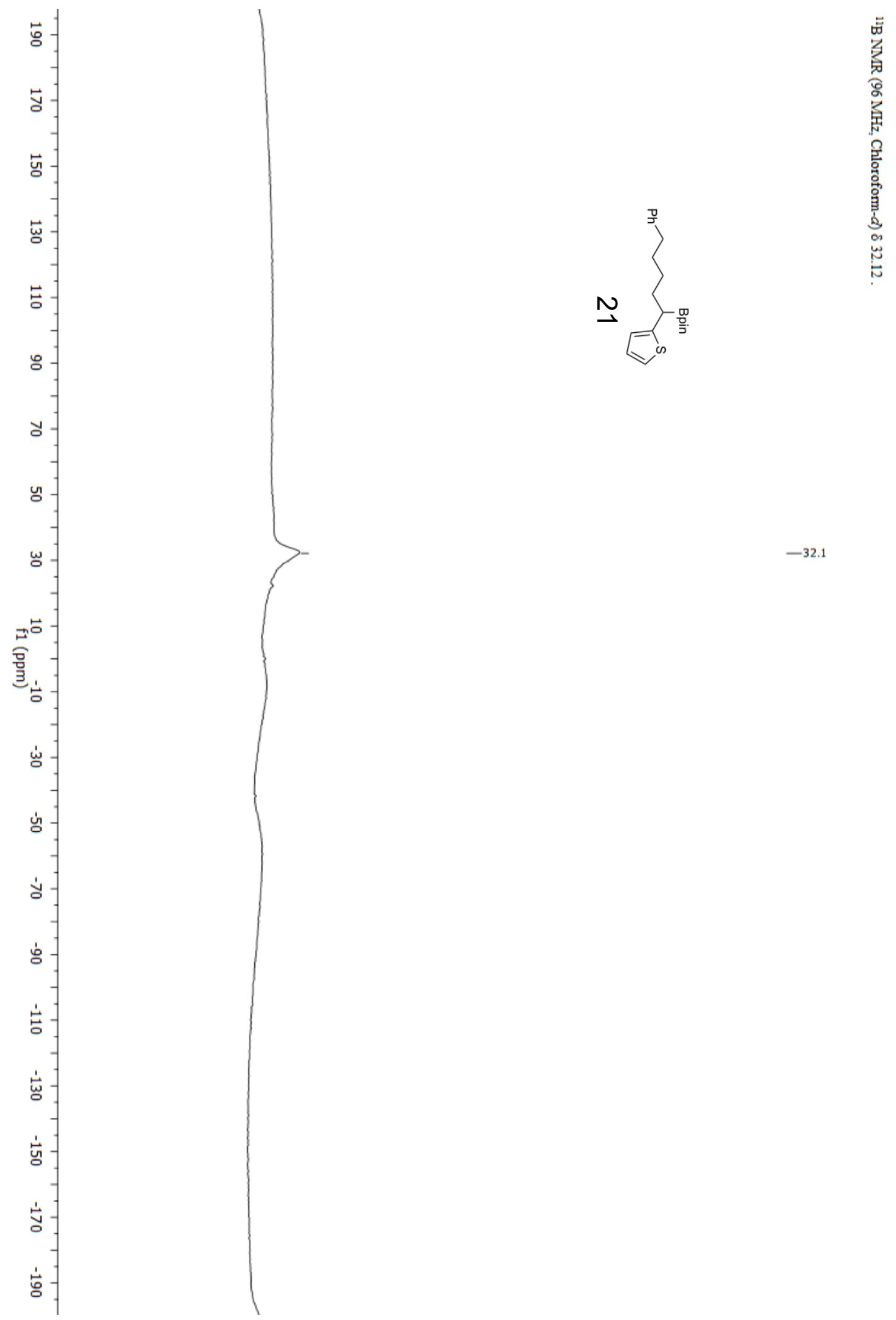

$-32.1$ 


$$
\mid
$$




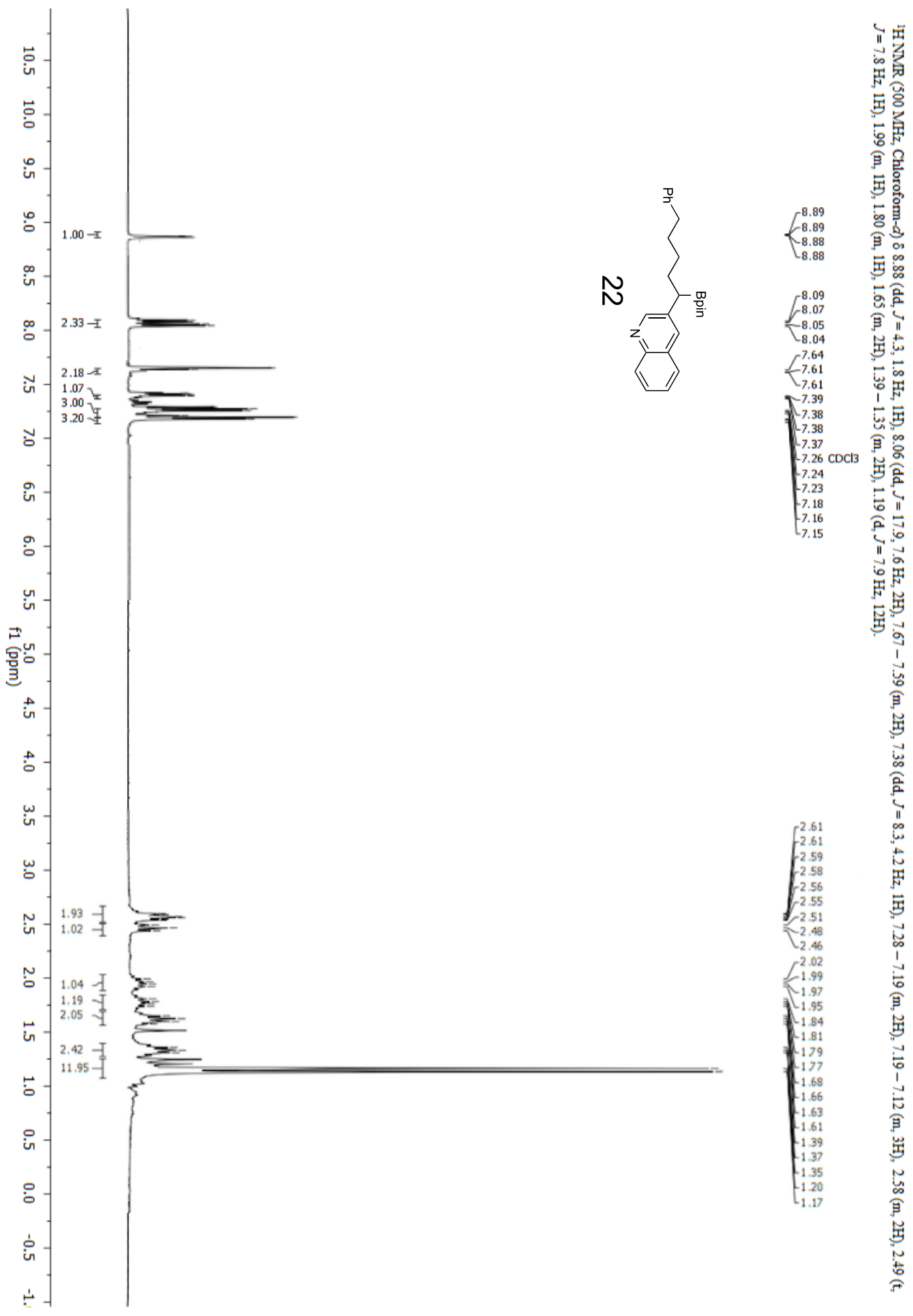



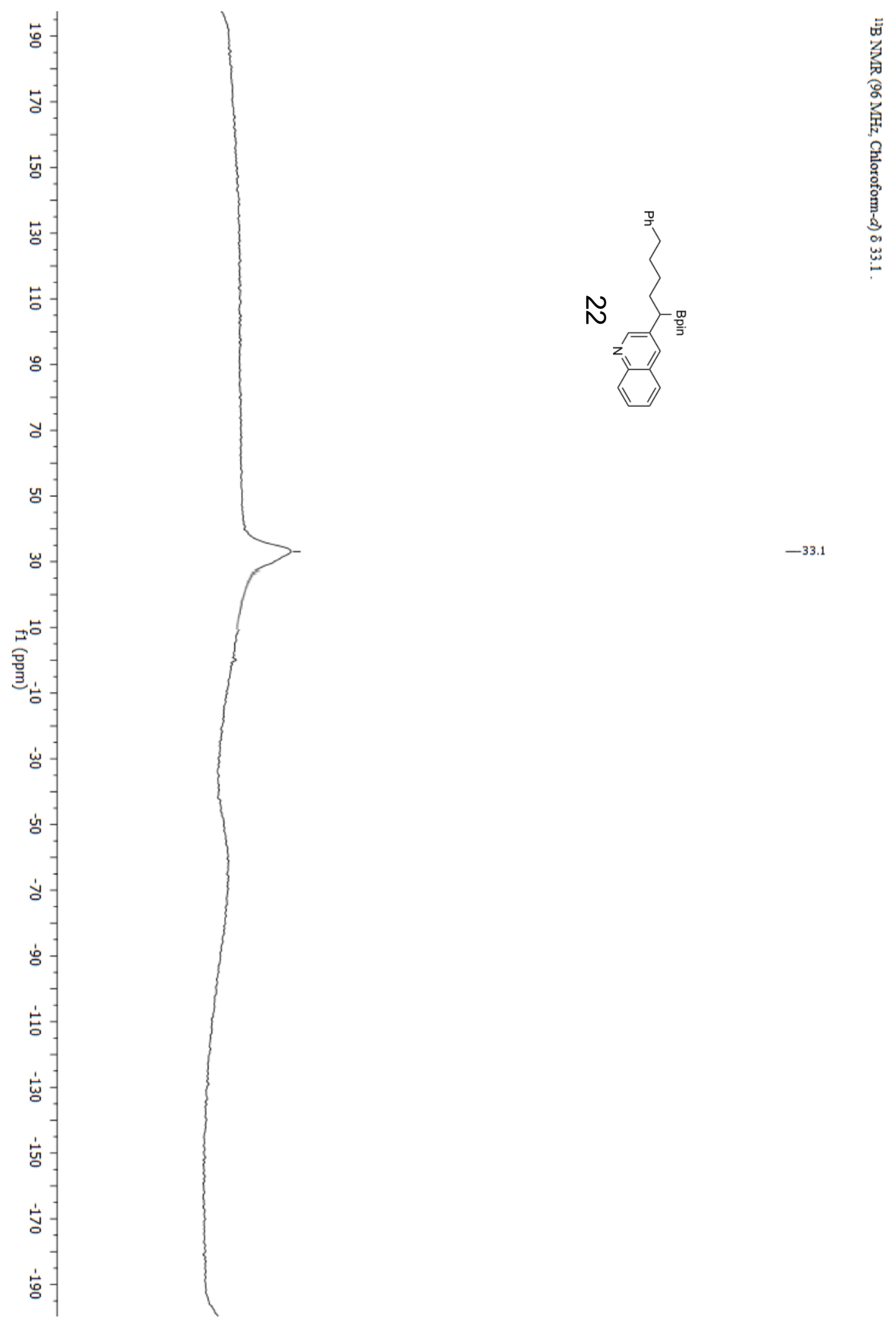

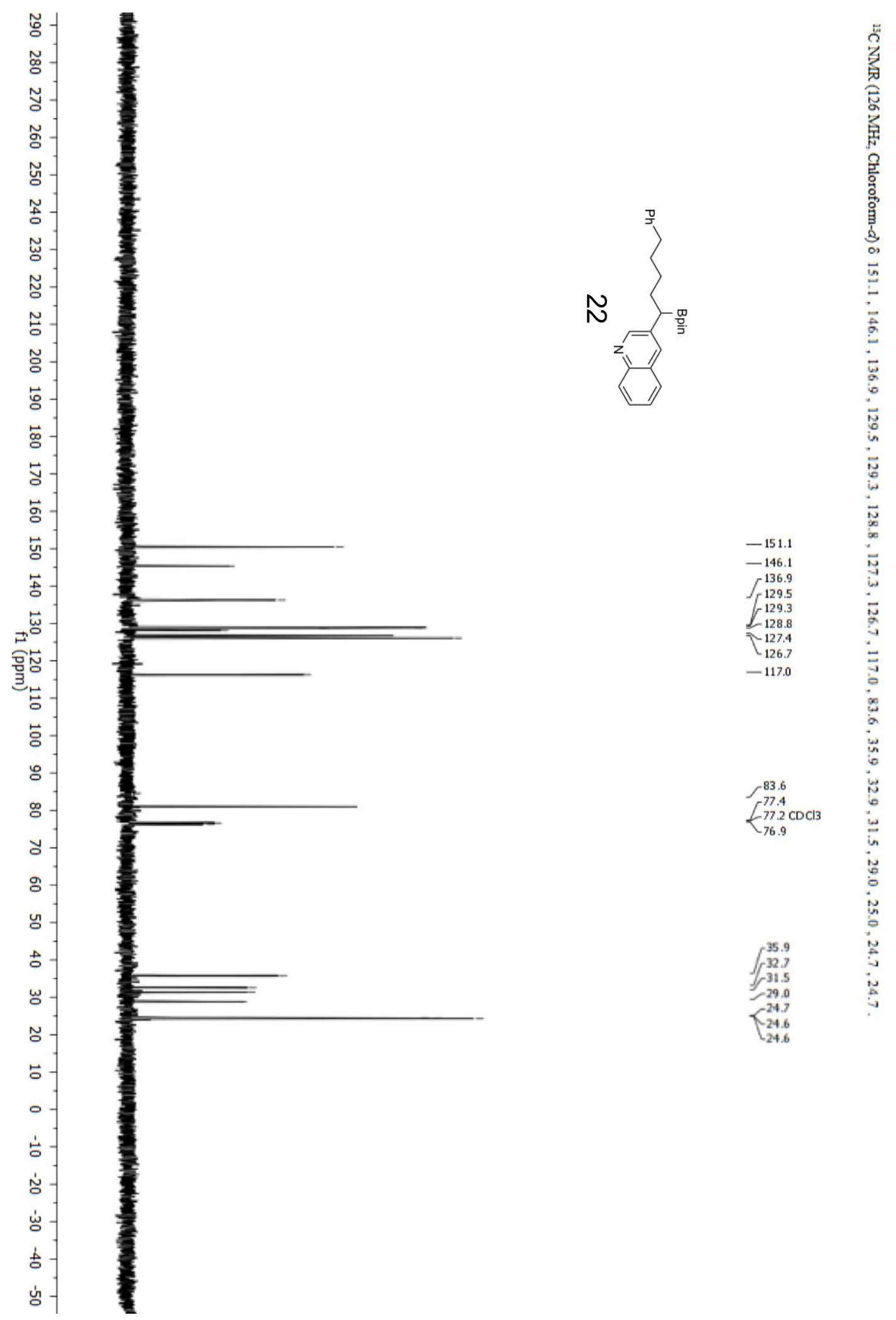


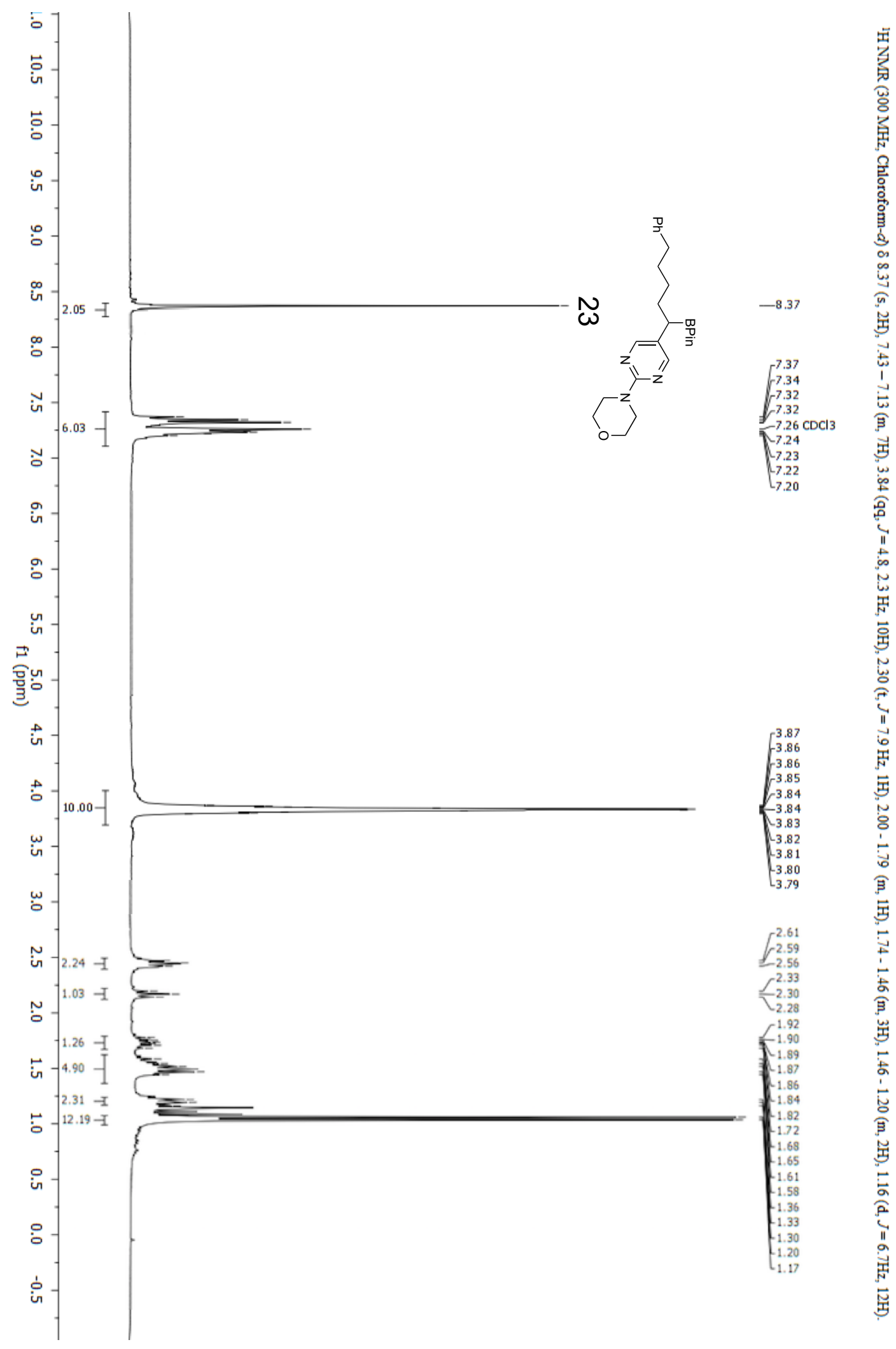



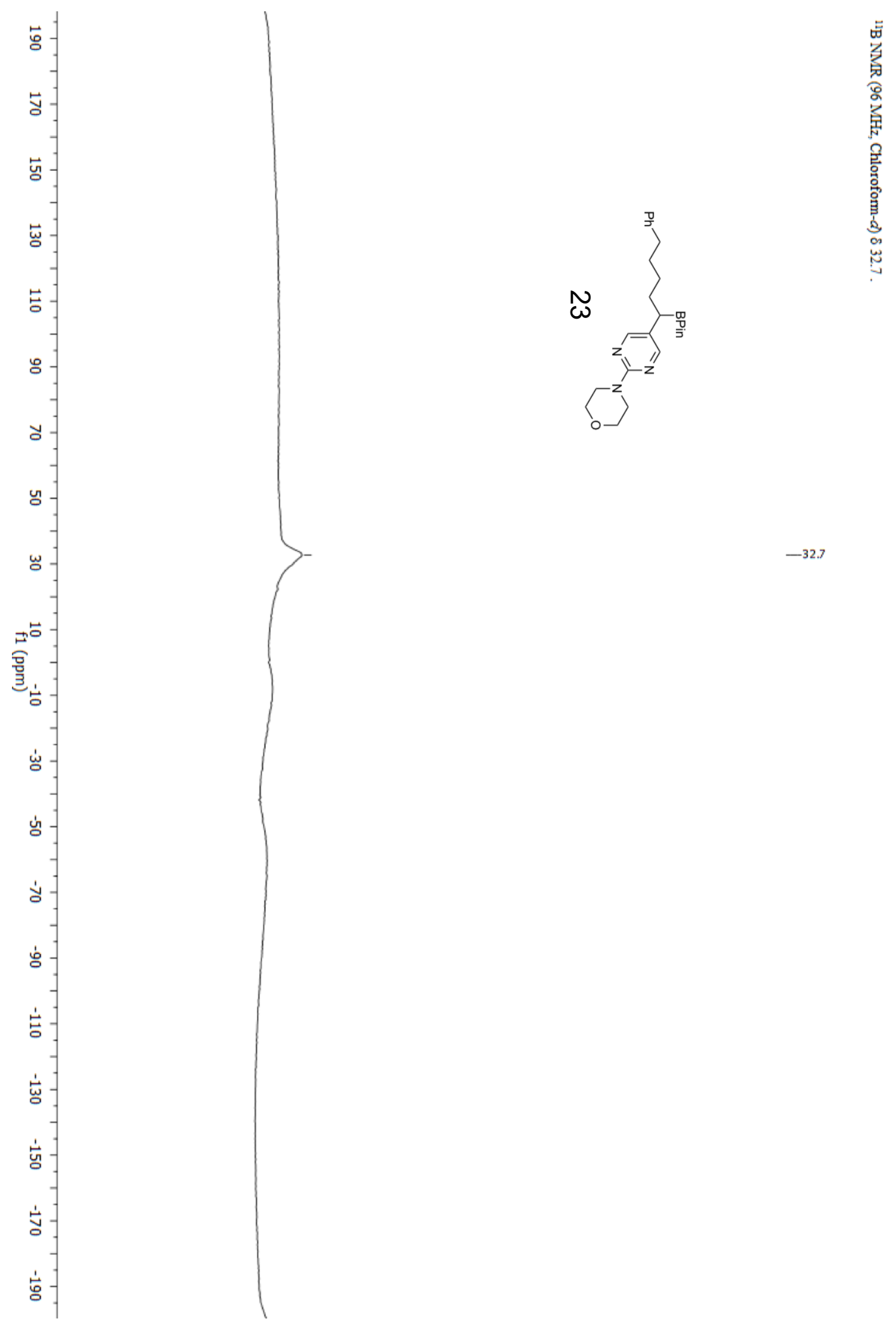

$-32.7$ 


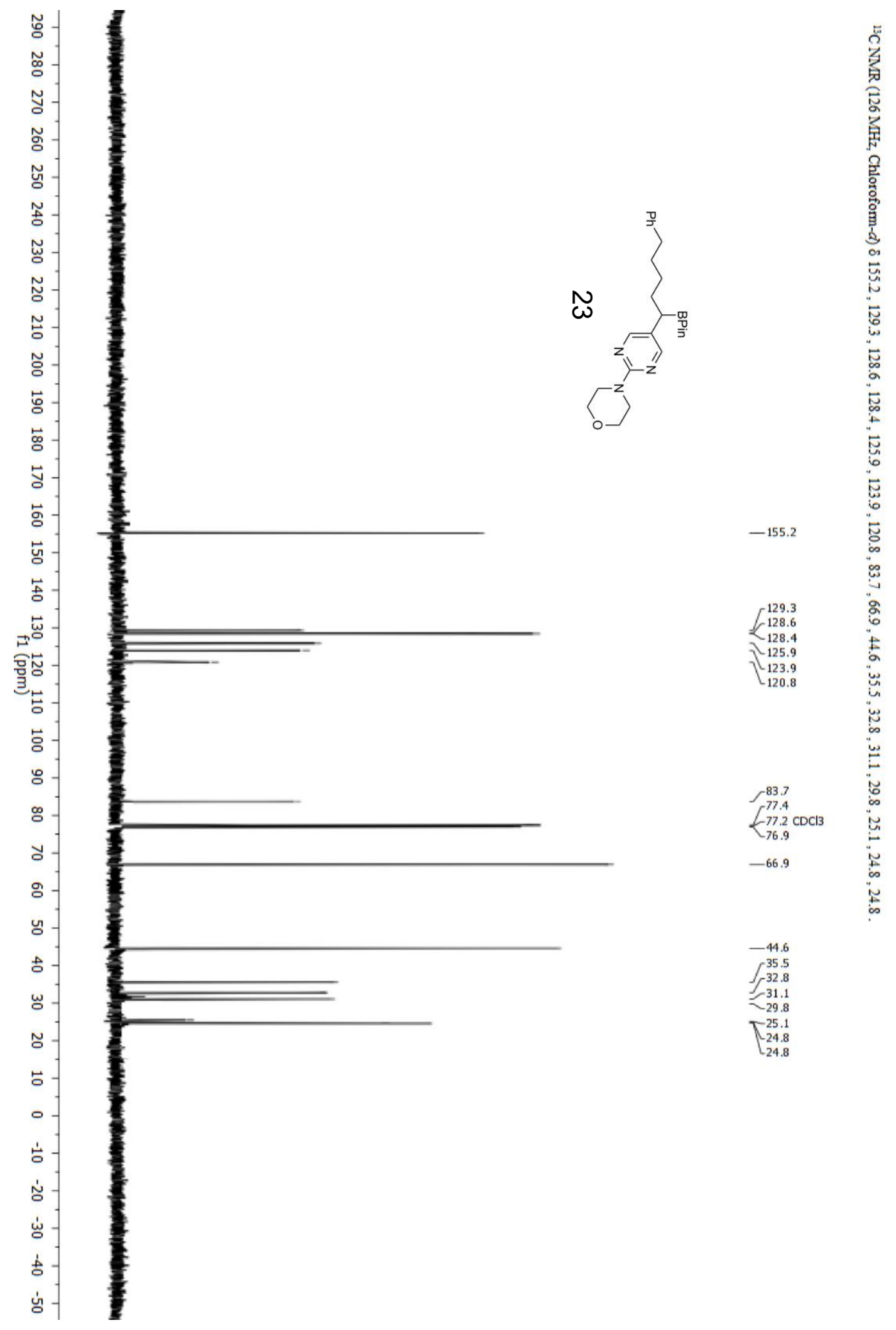




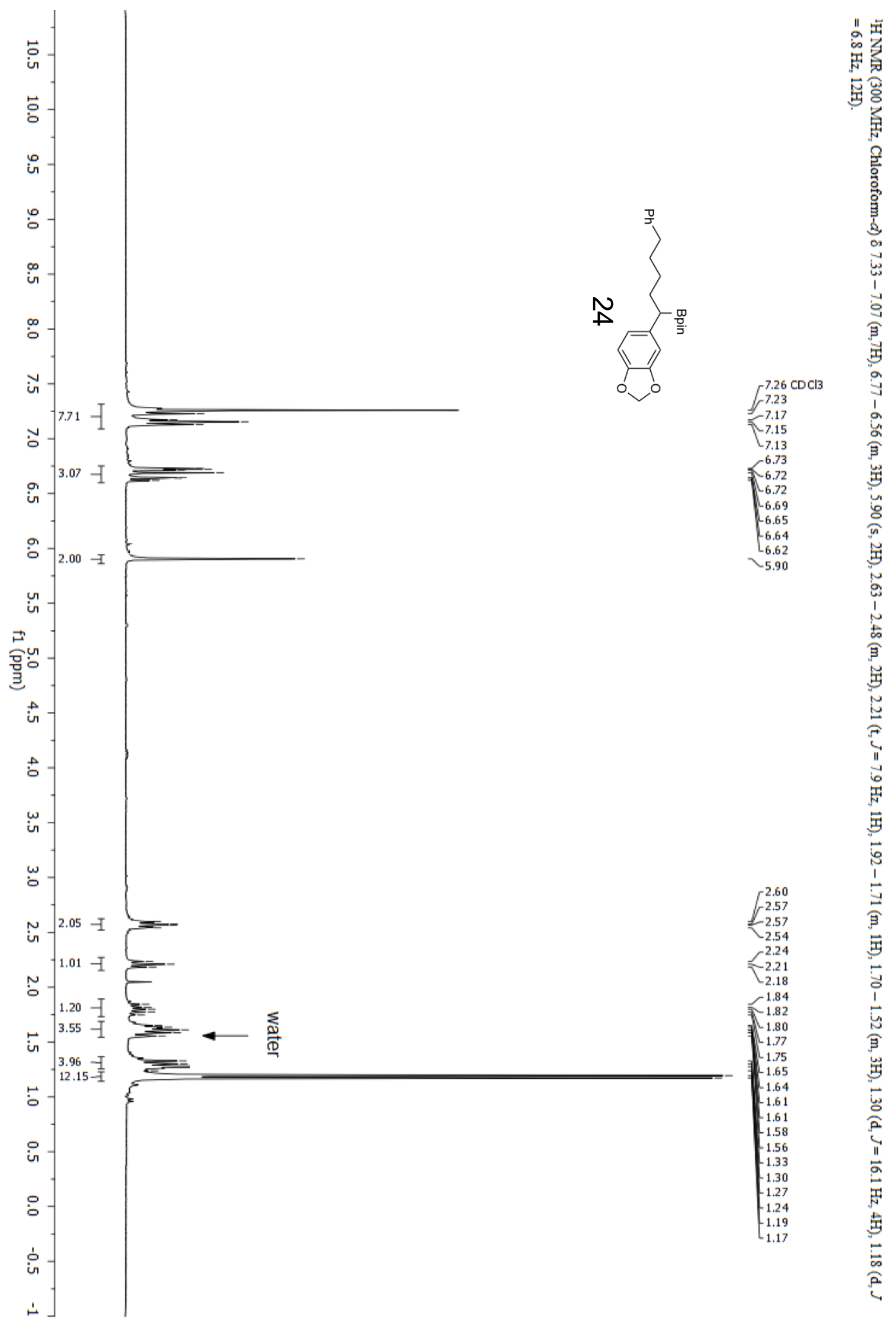

S82 

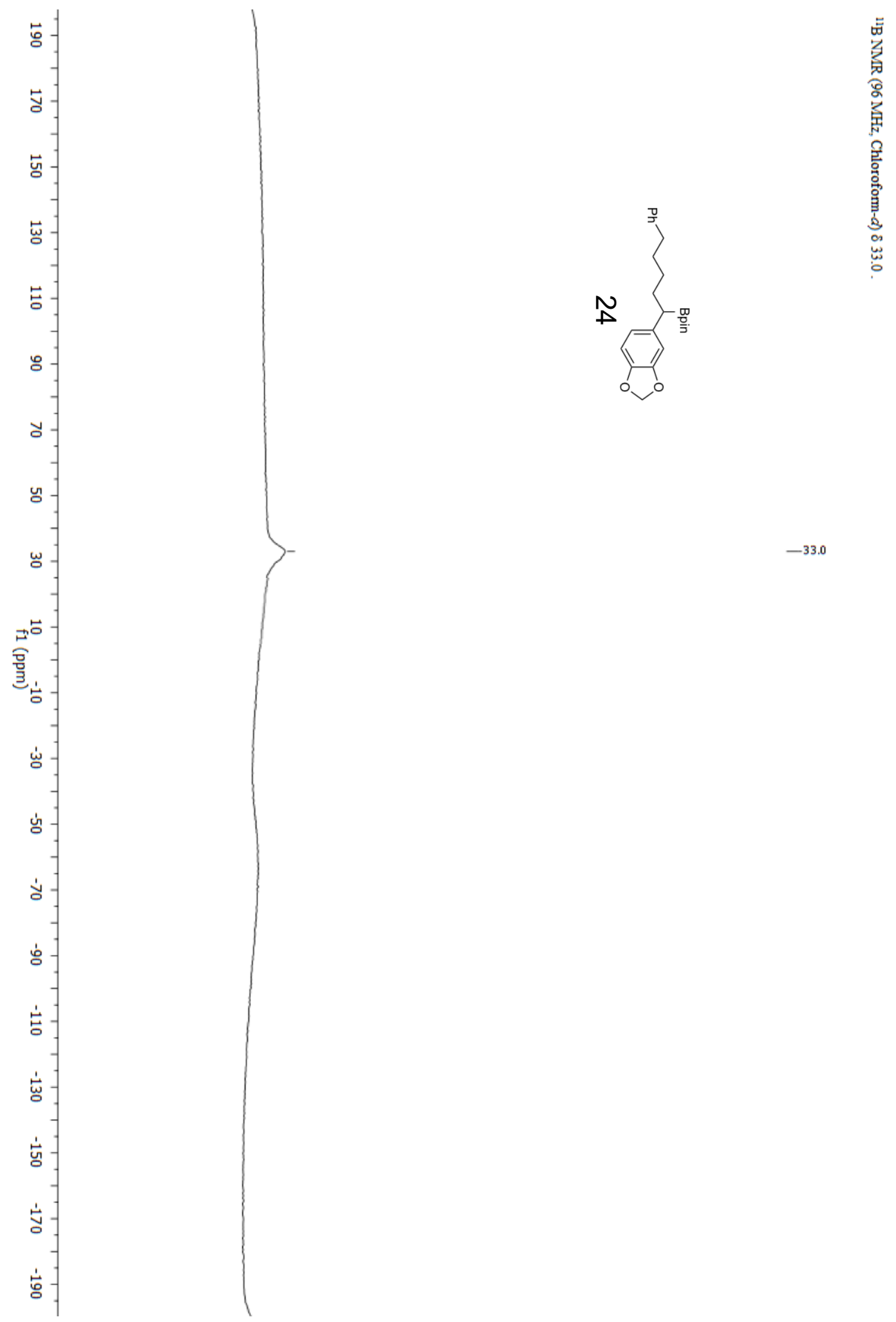


$$
\mid
$$




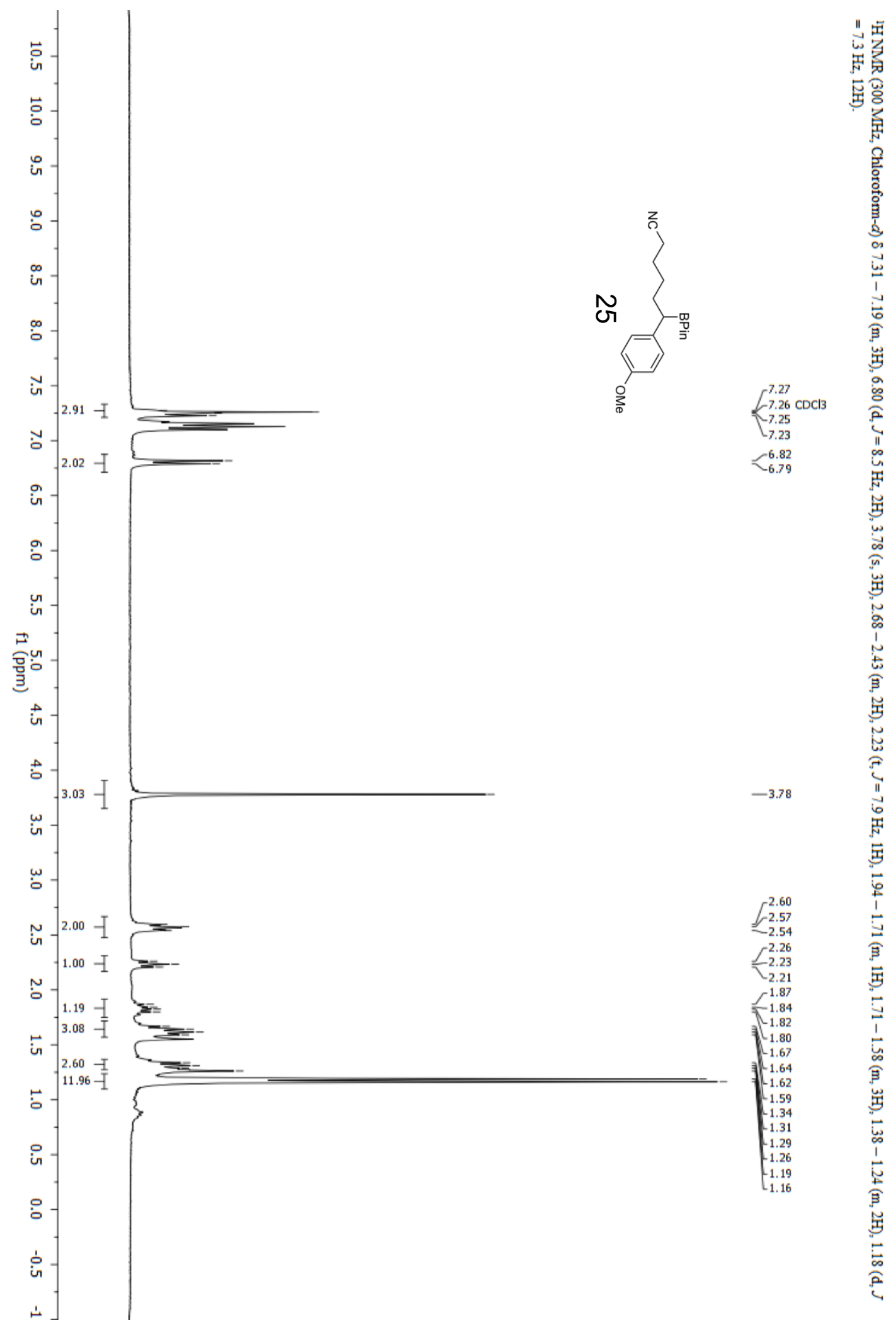



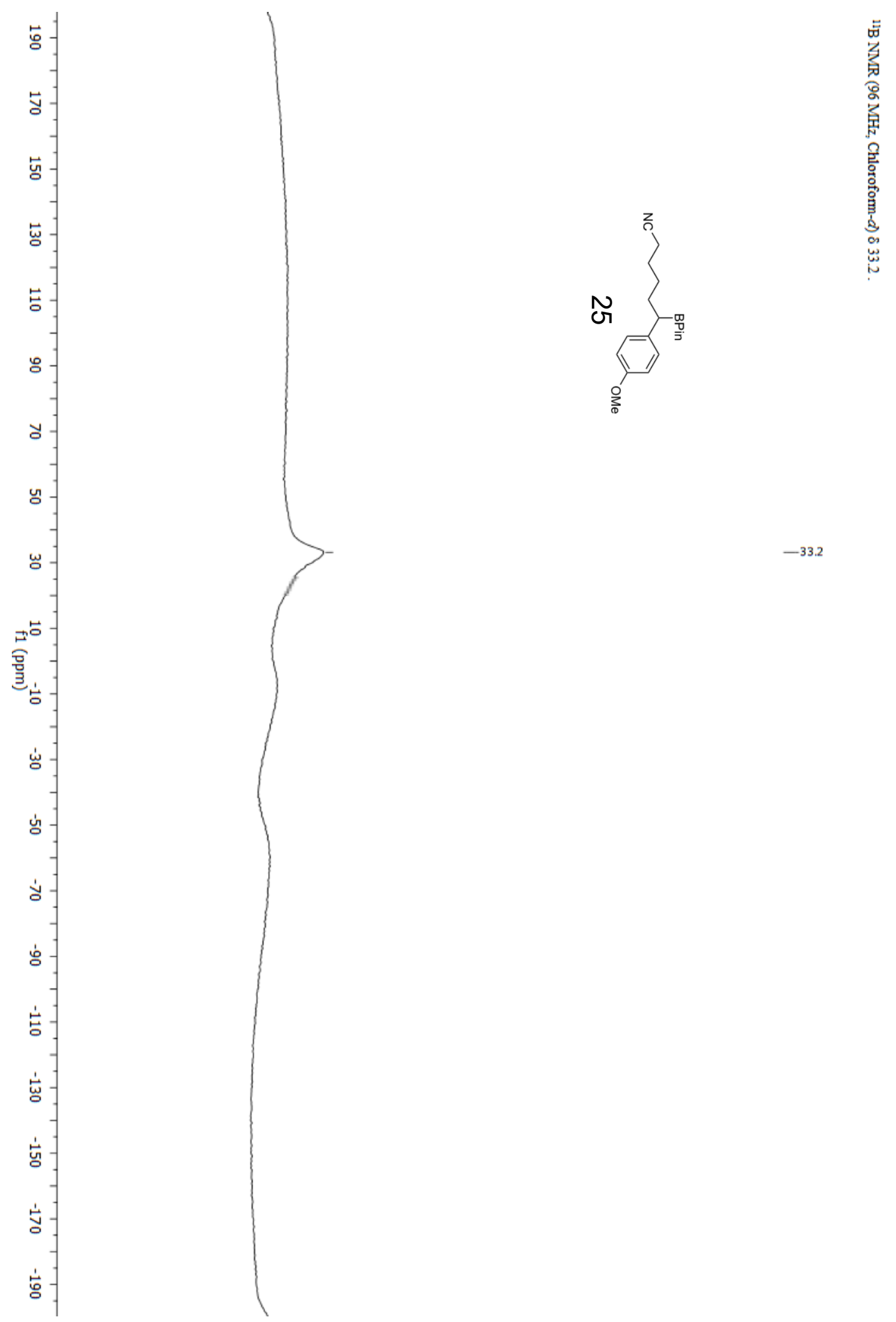

$-33.2$ 


$$
\text { | }
$$




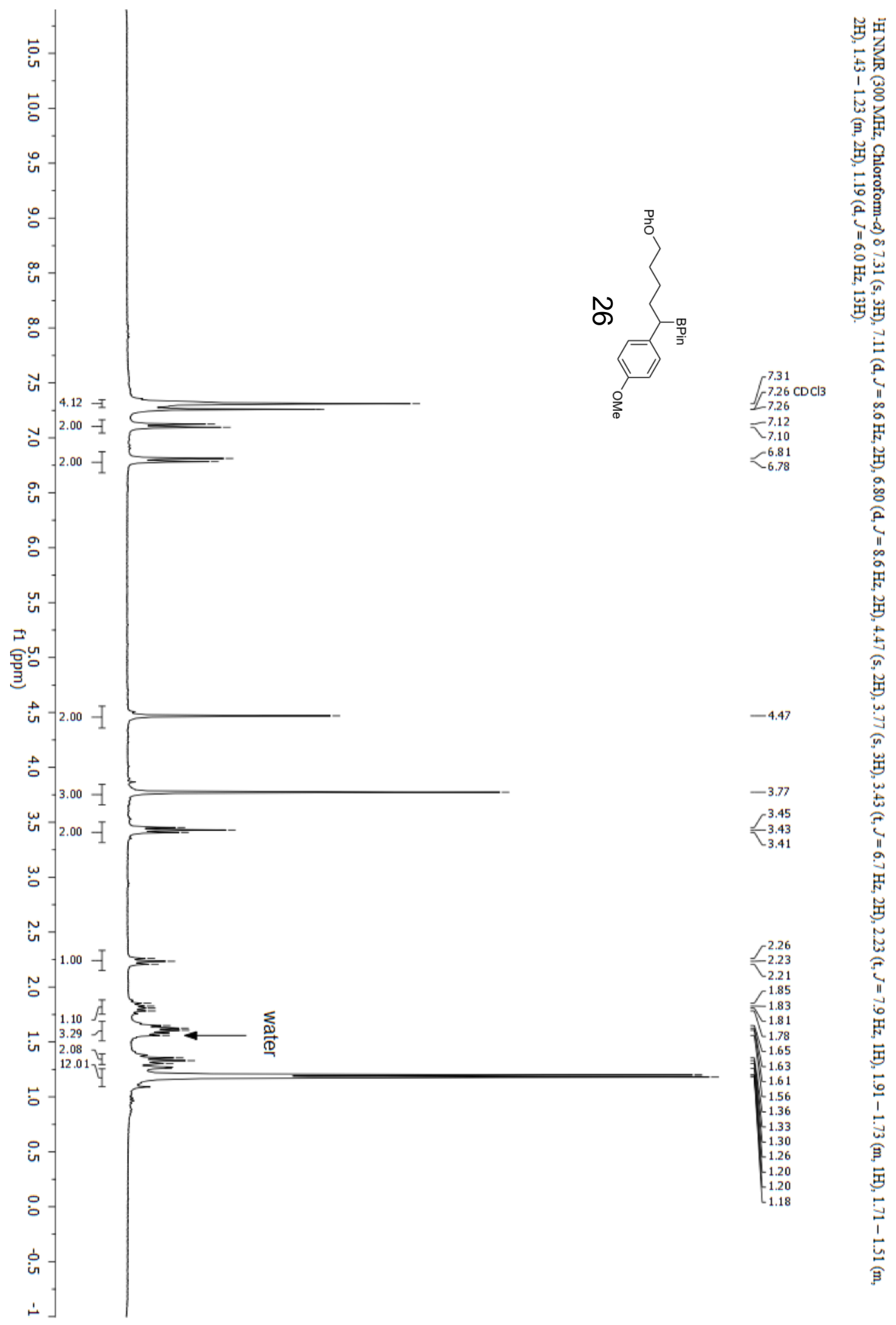

S88 

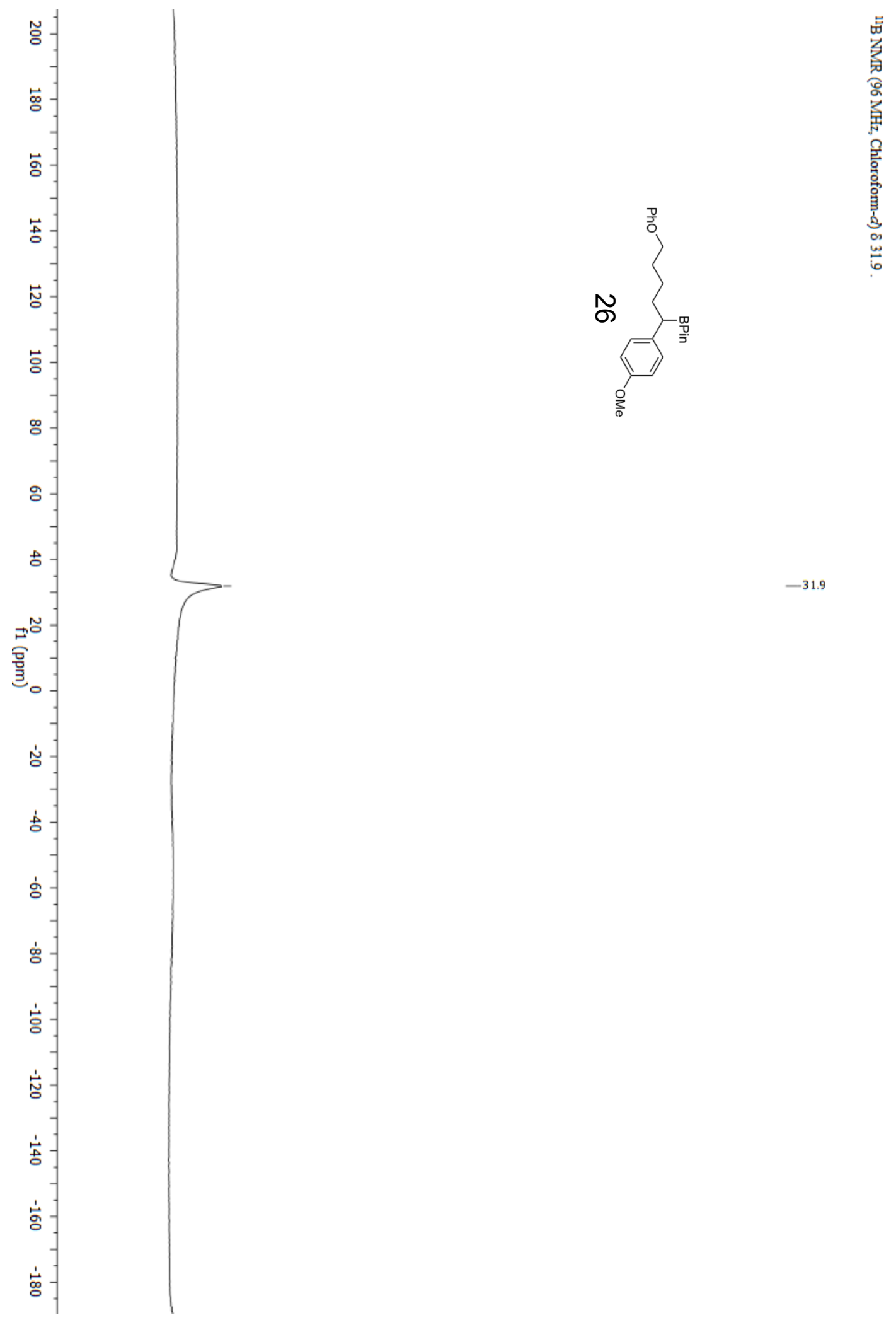

$-31.9$ 


$$
\mid
$$




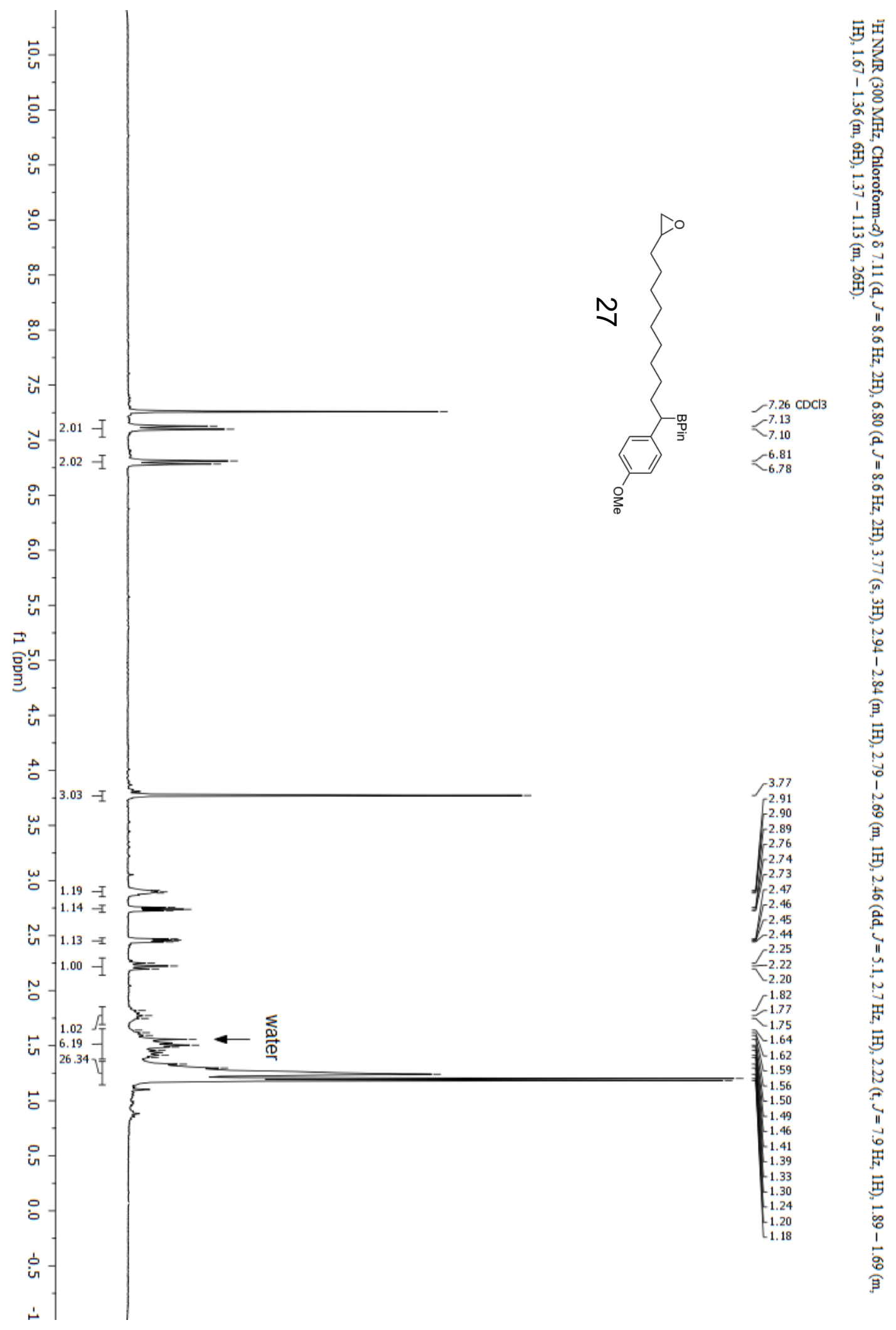



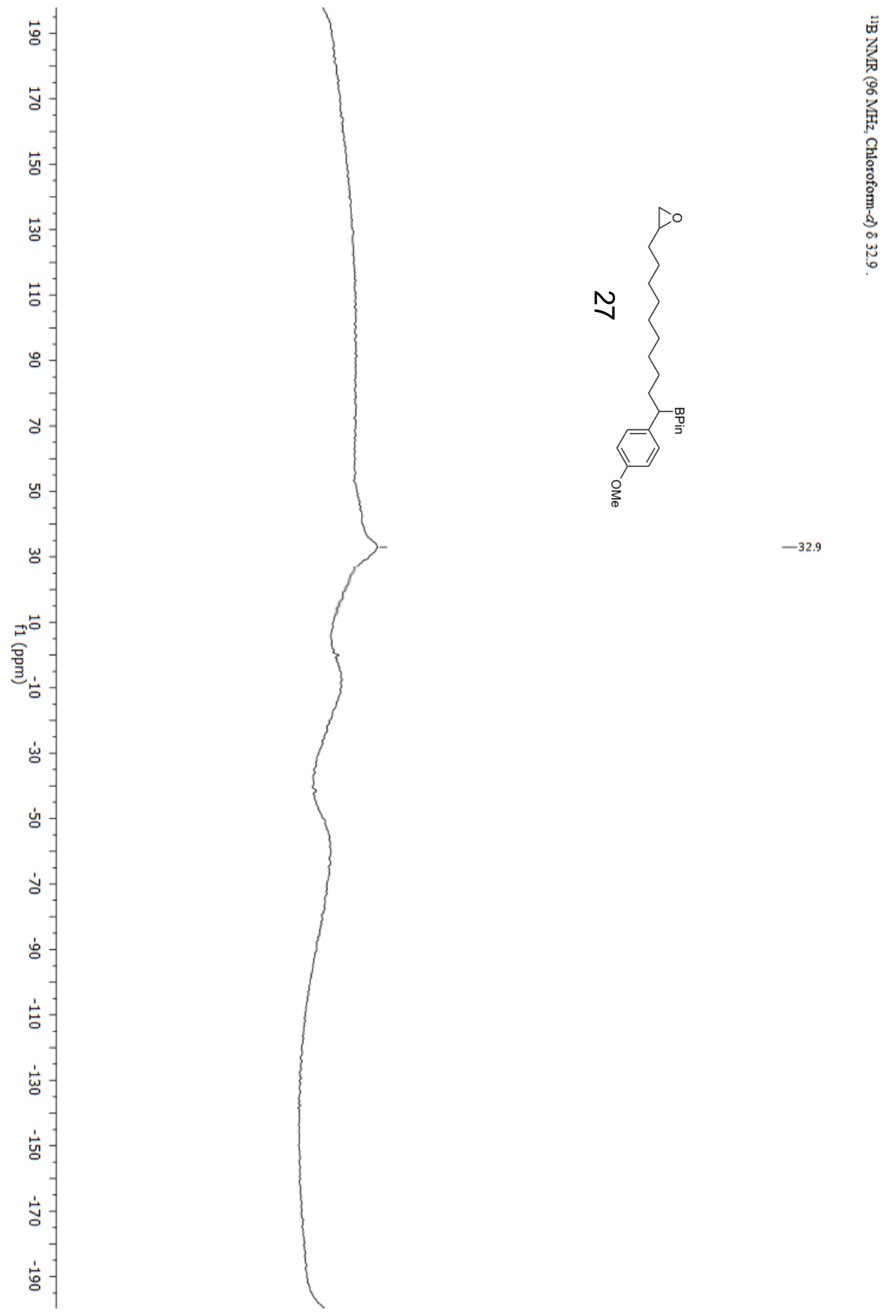


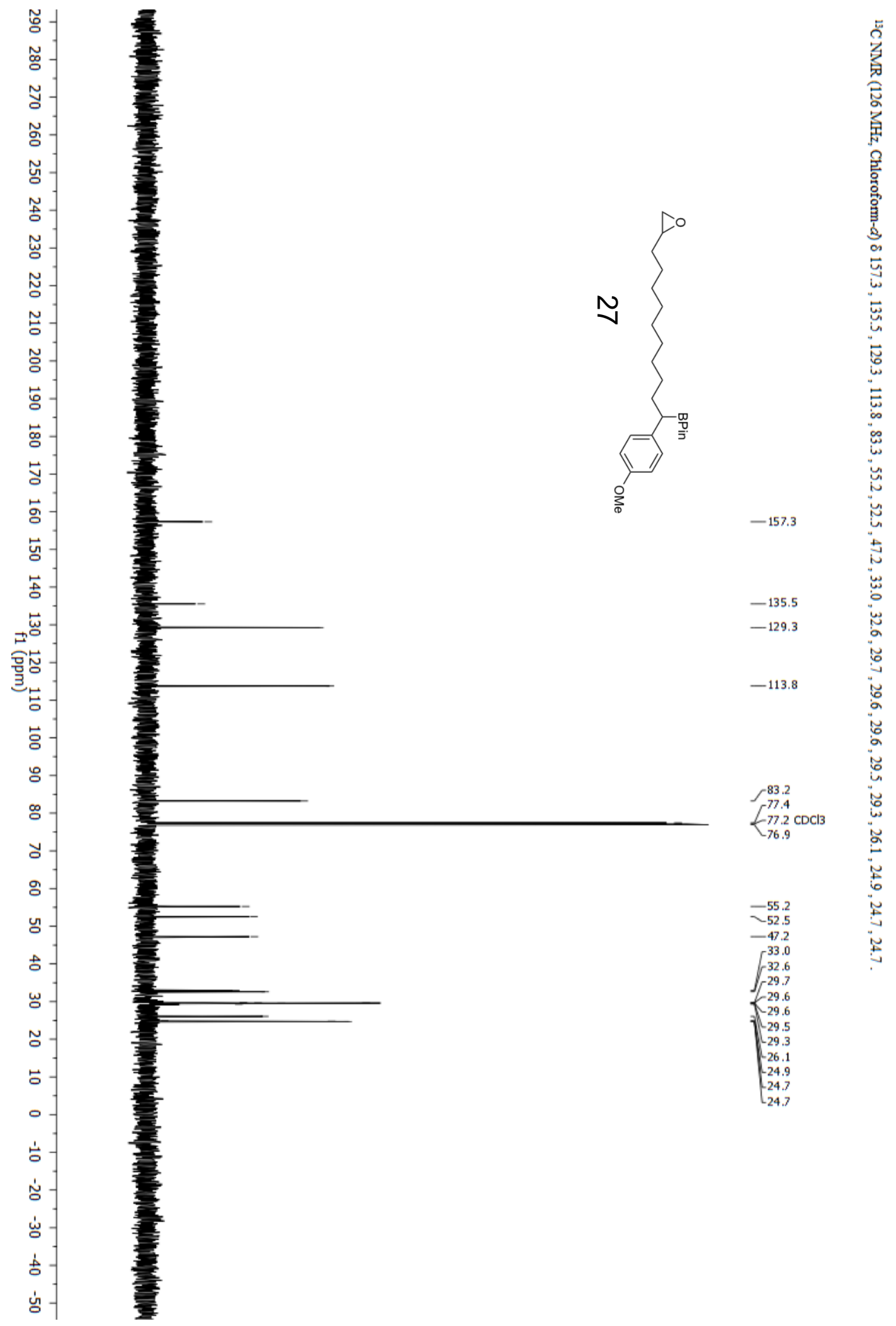



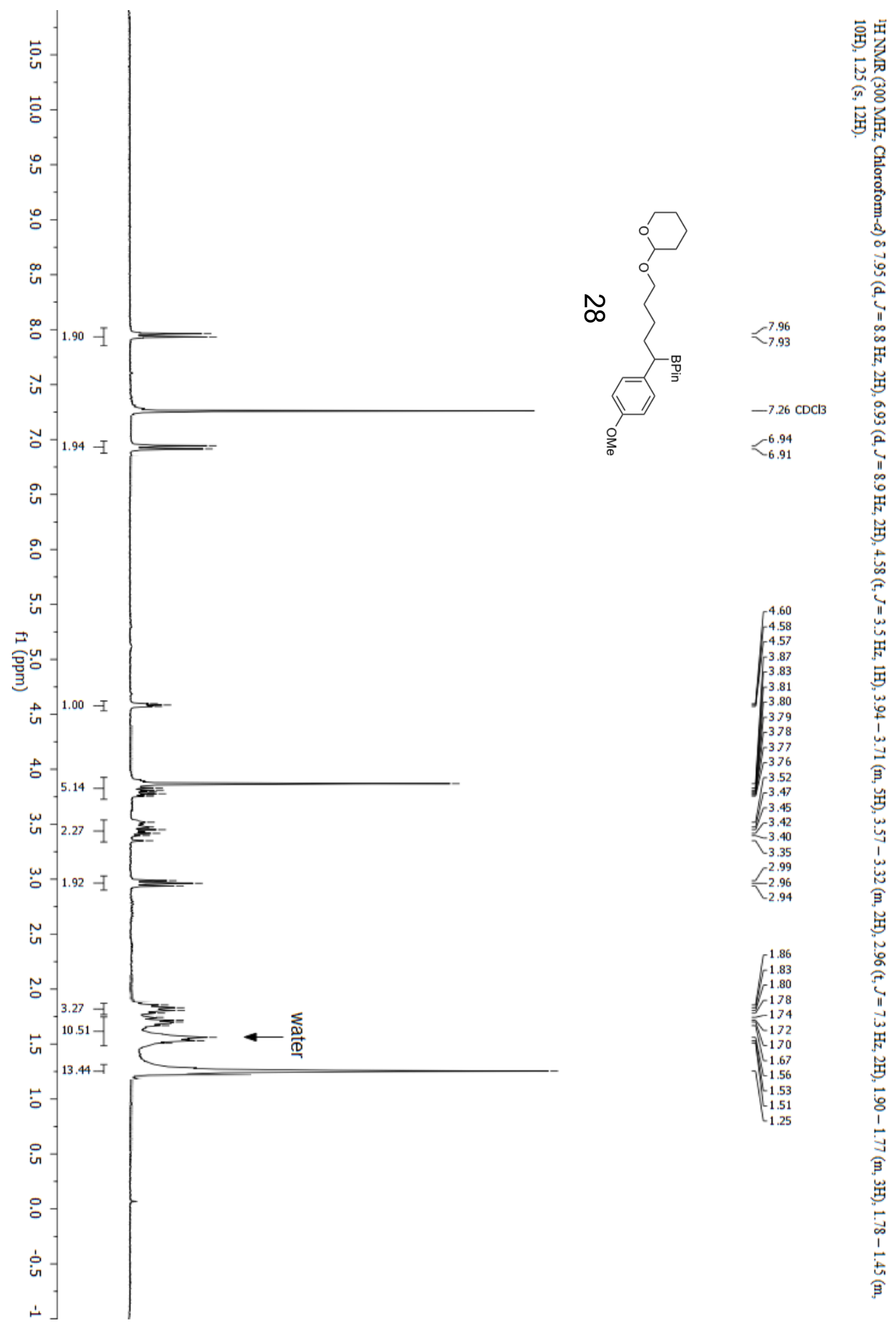

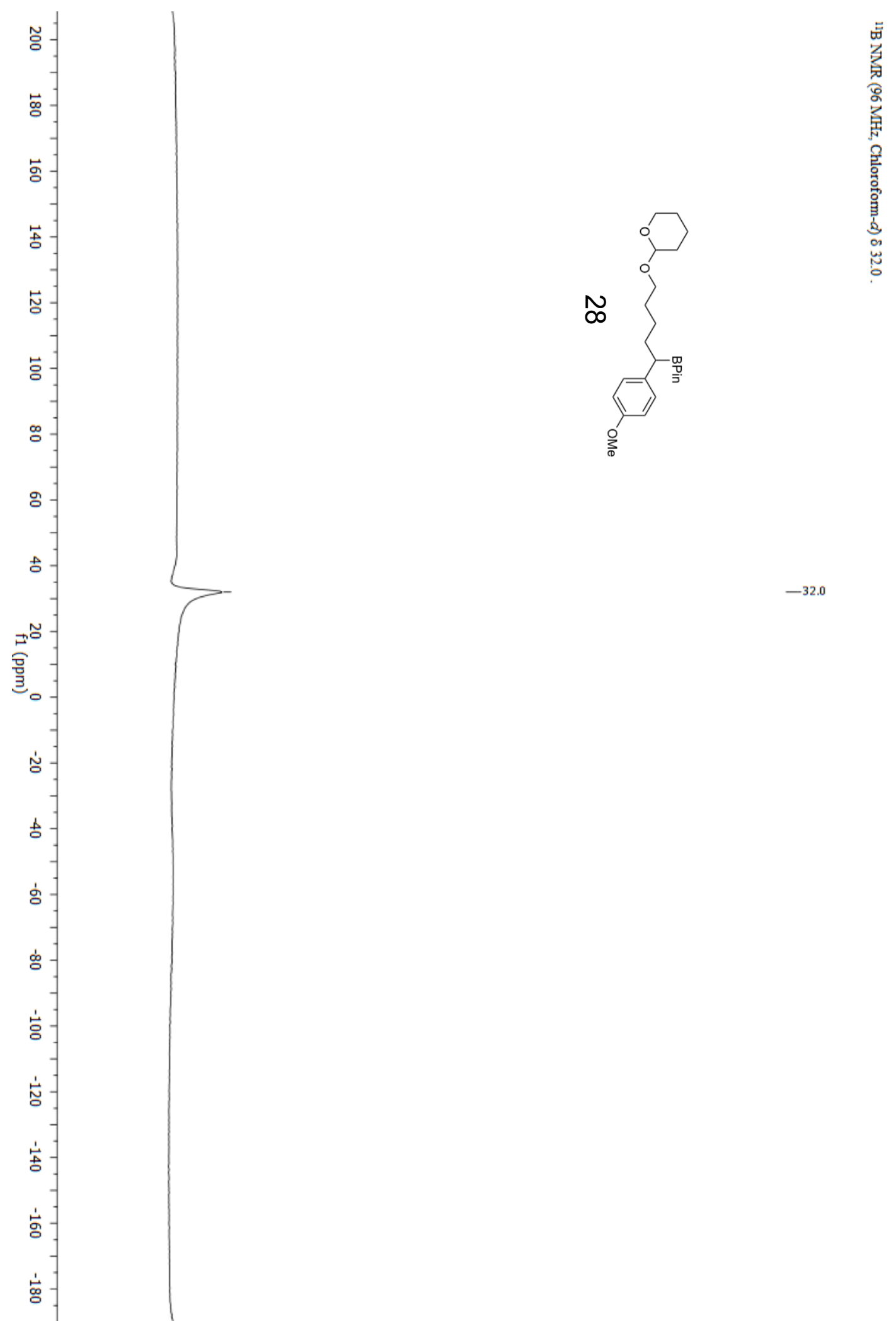


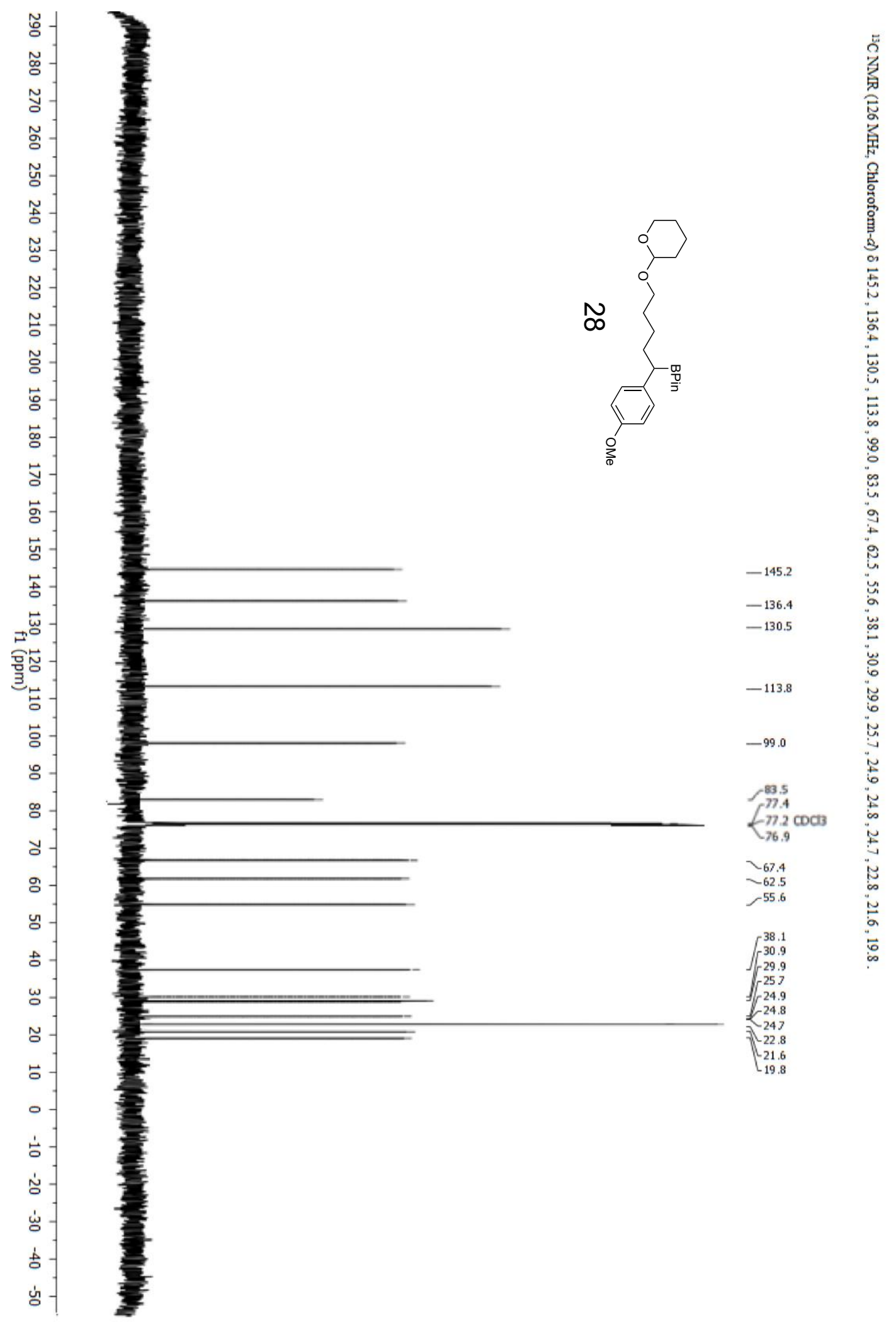




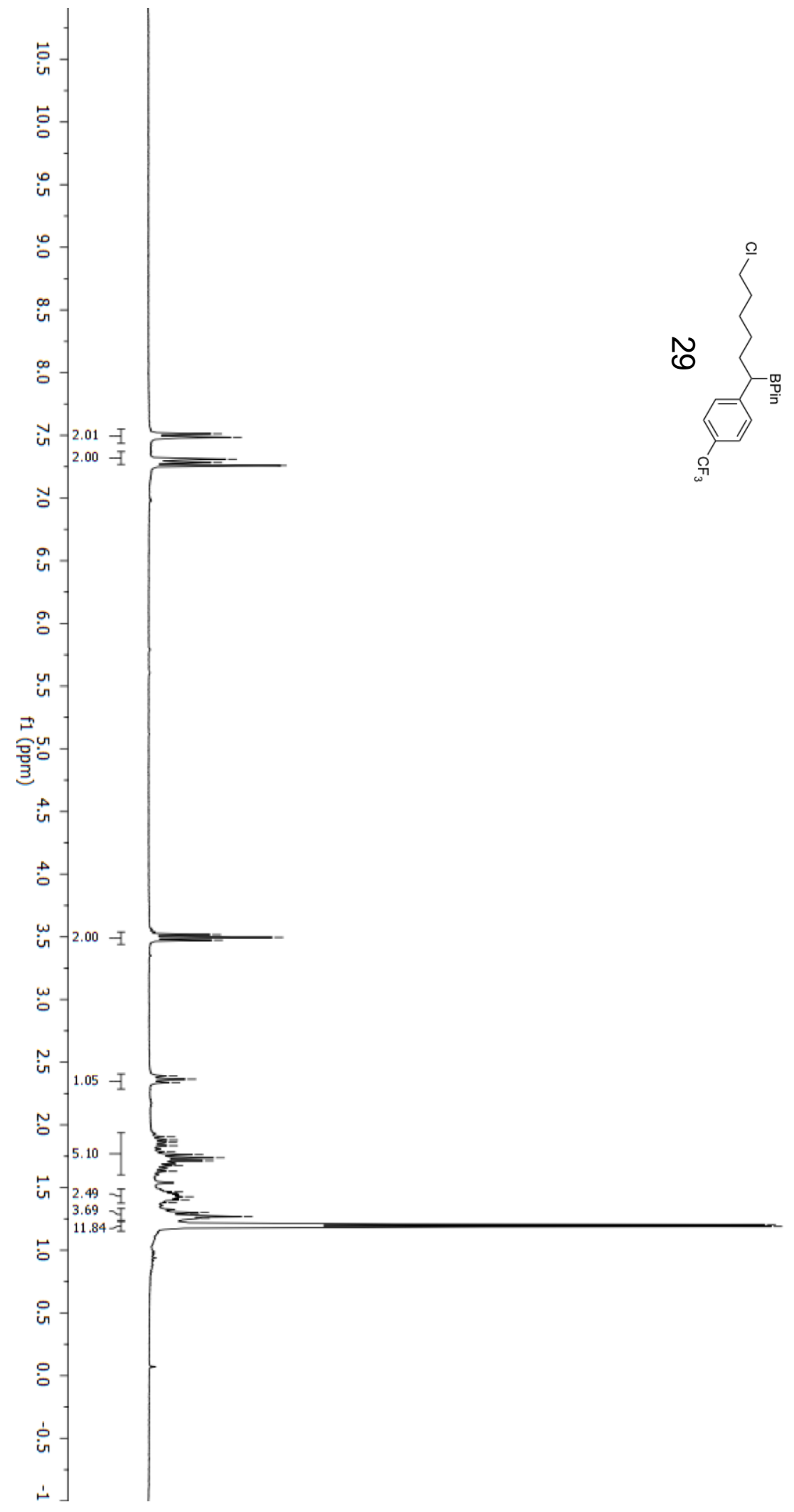

临密

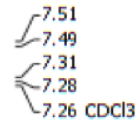




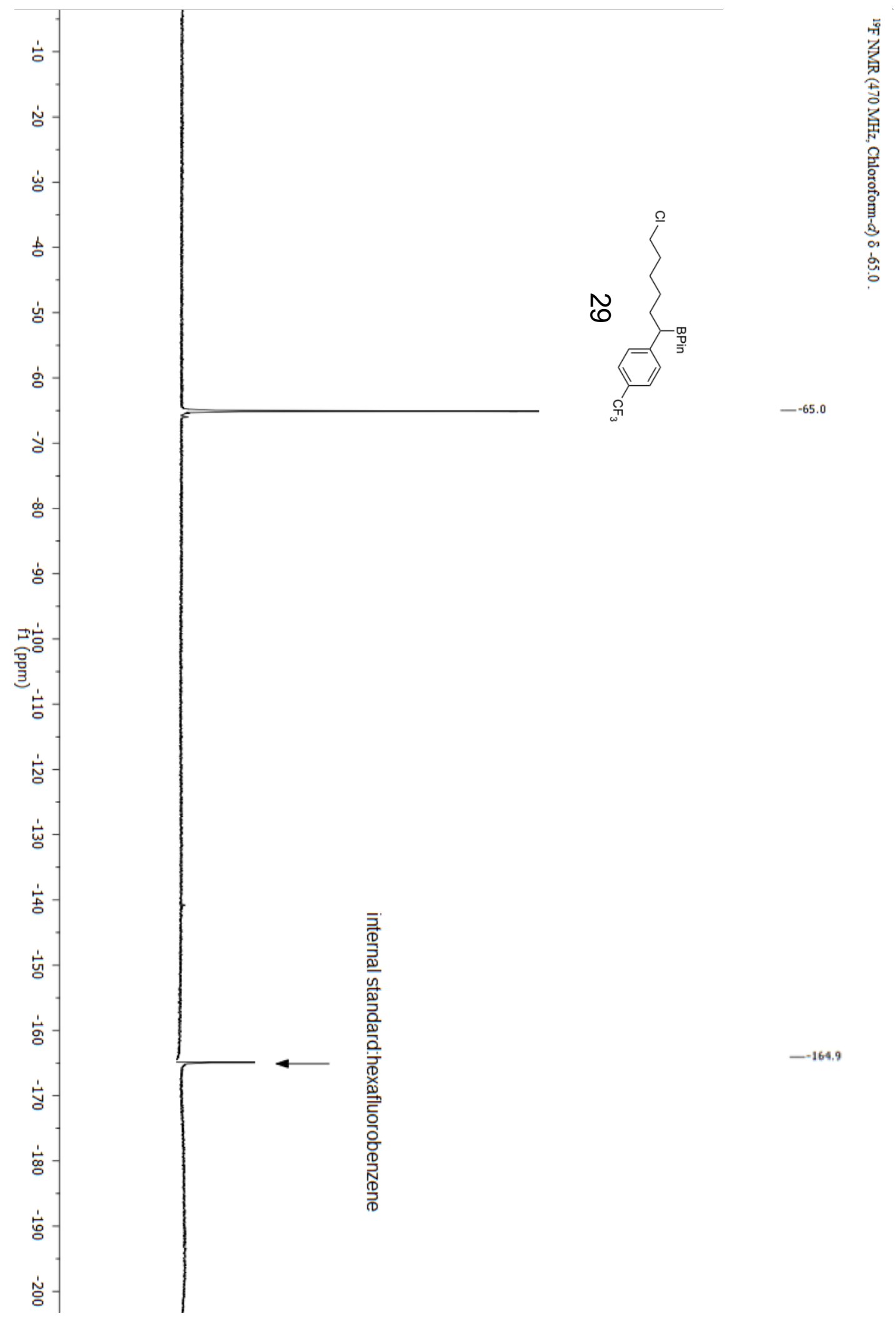



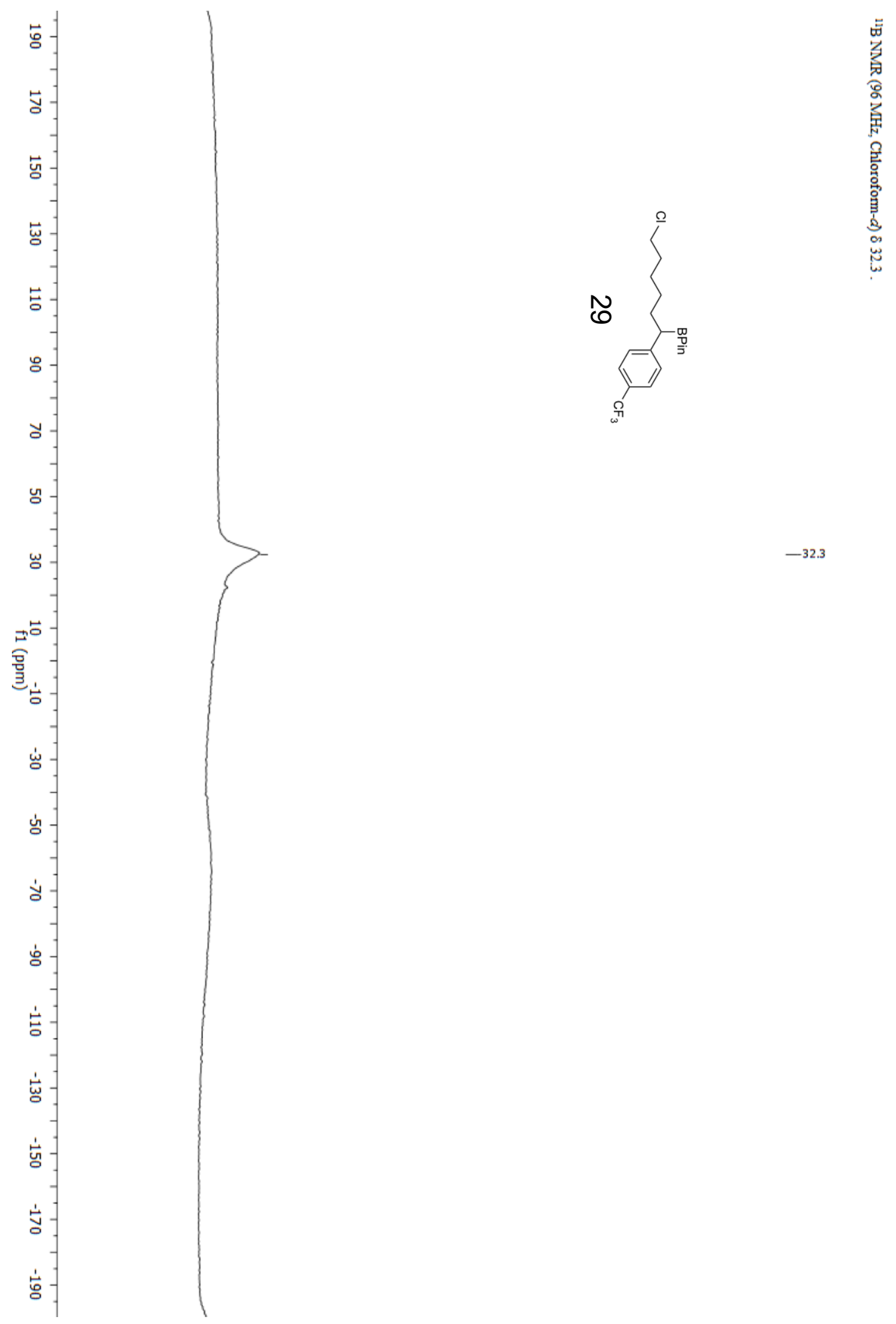

$-32.3$ 


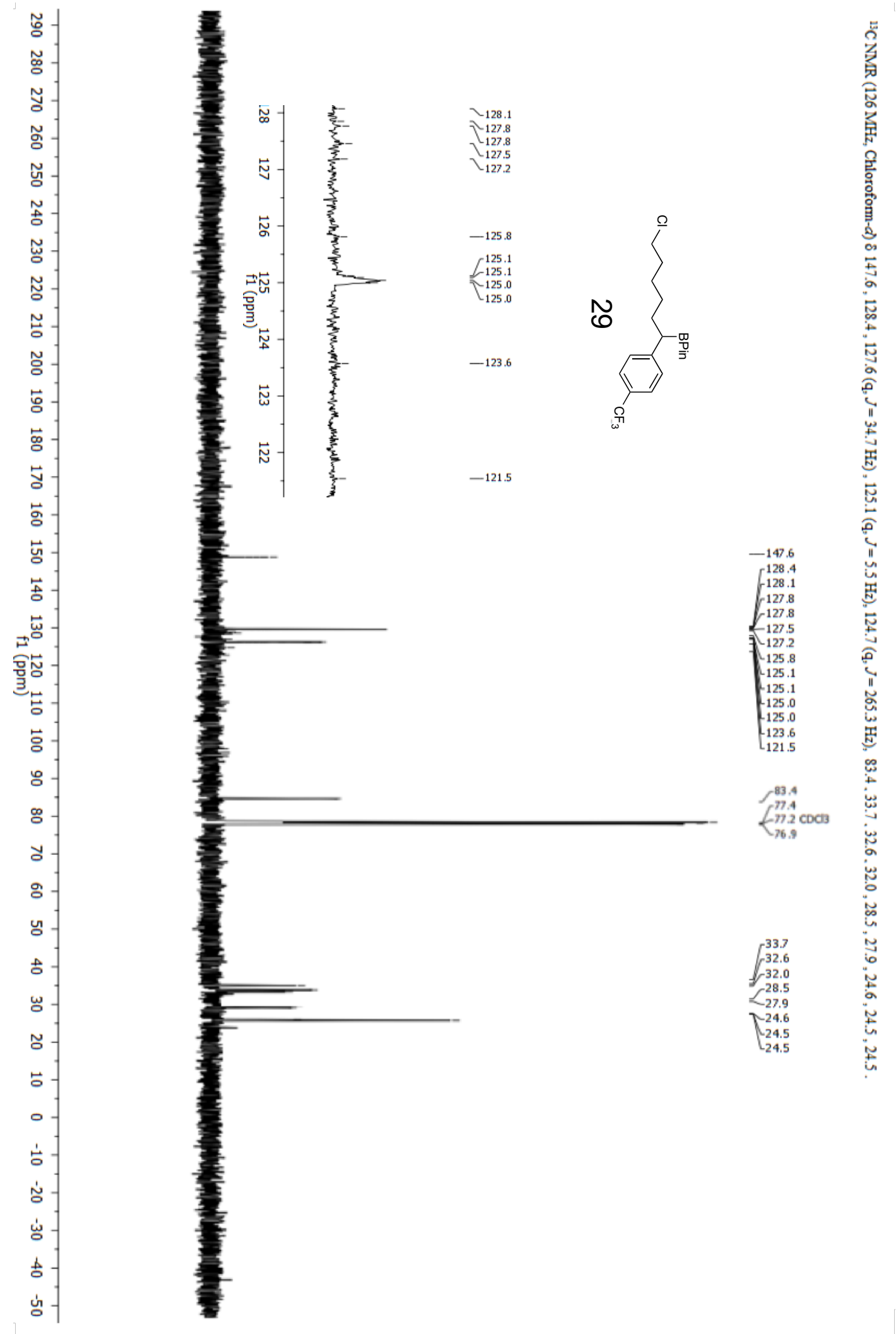



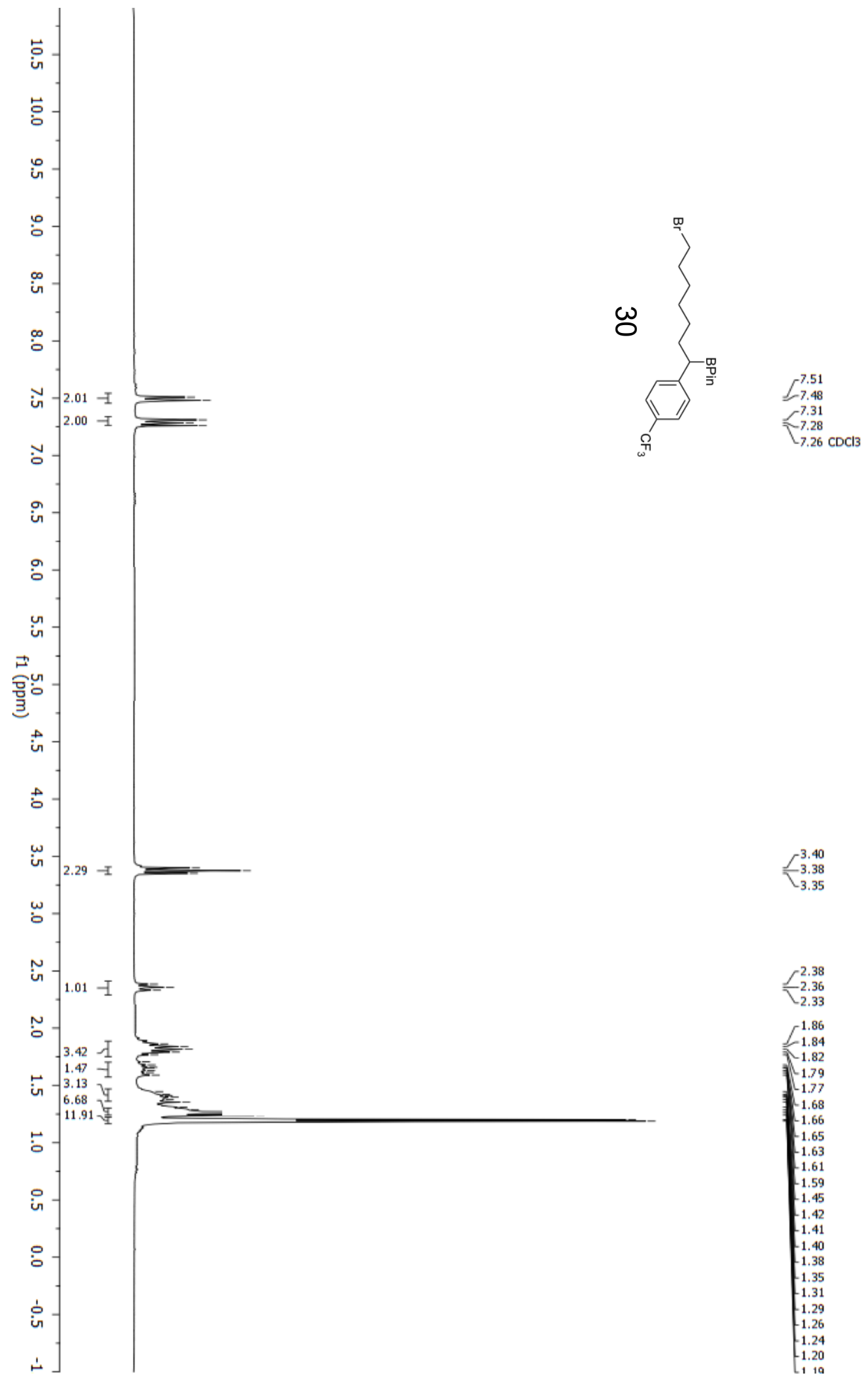

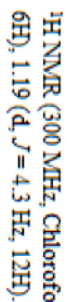

完易

눔

돌

岀兽

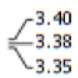

孚 

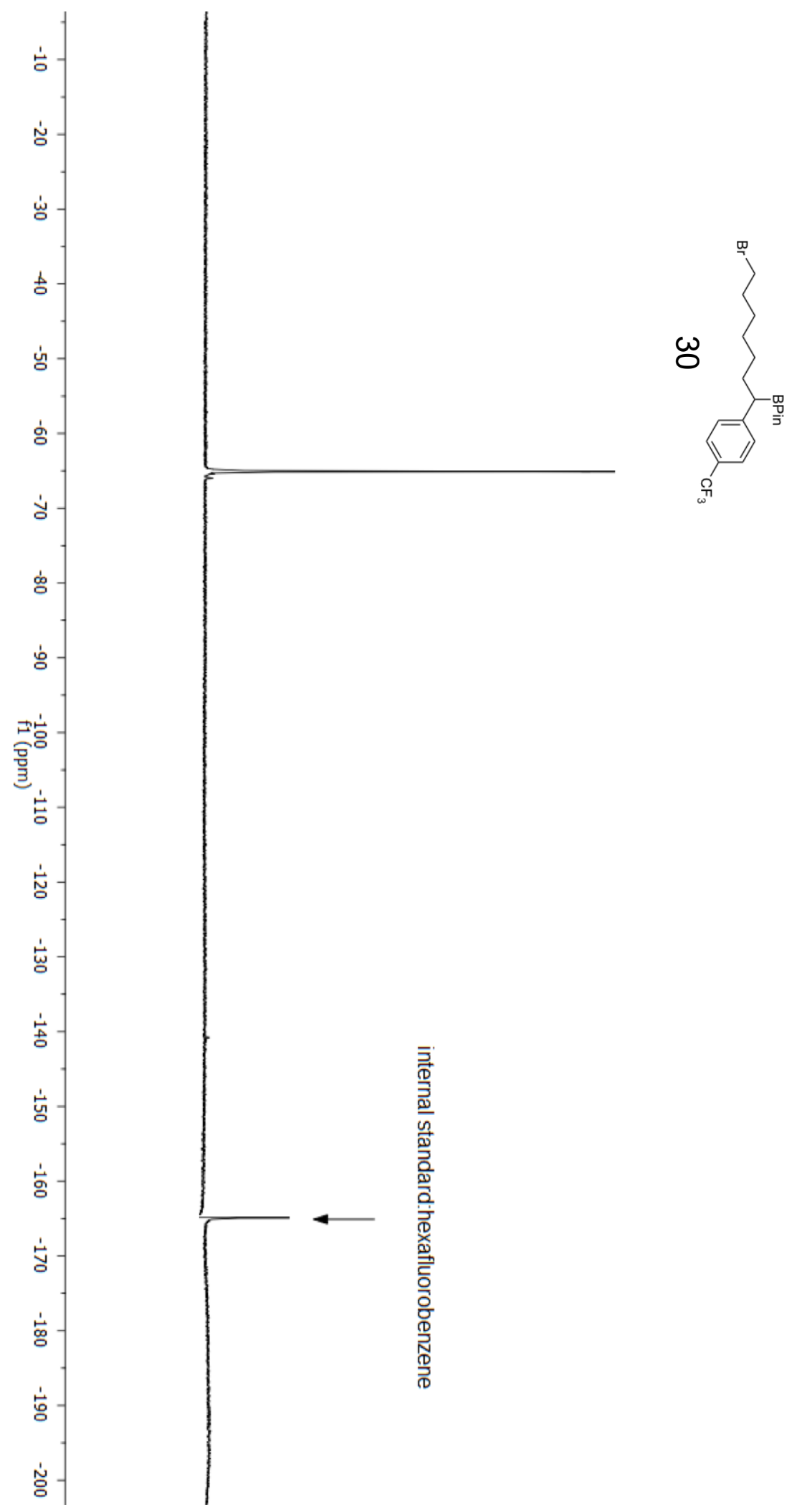

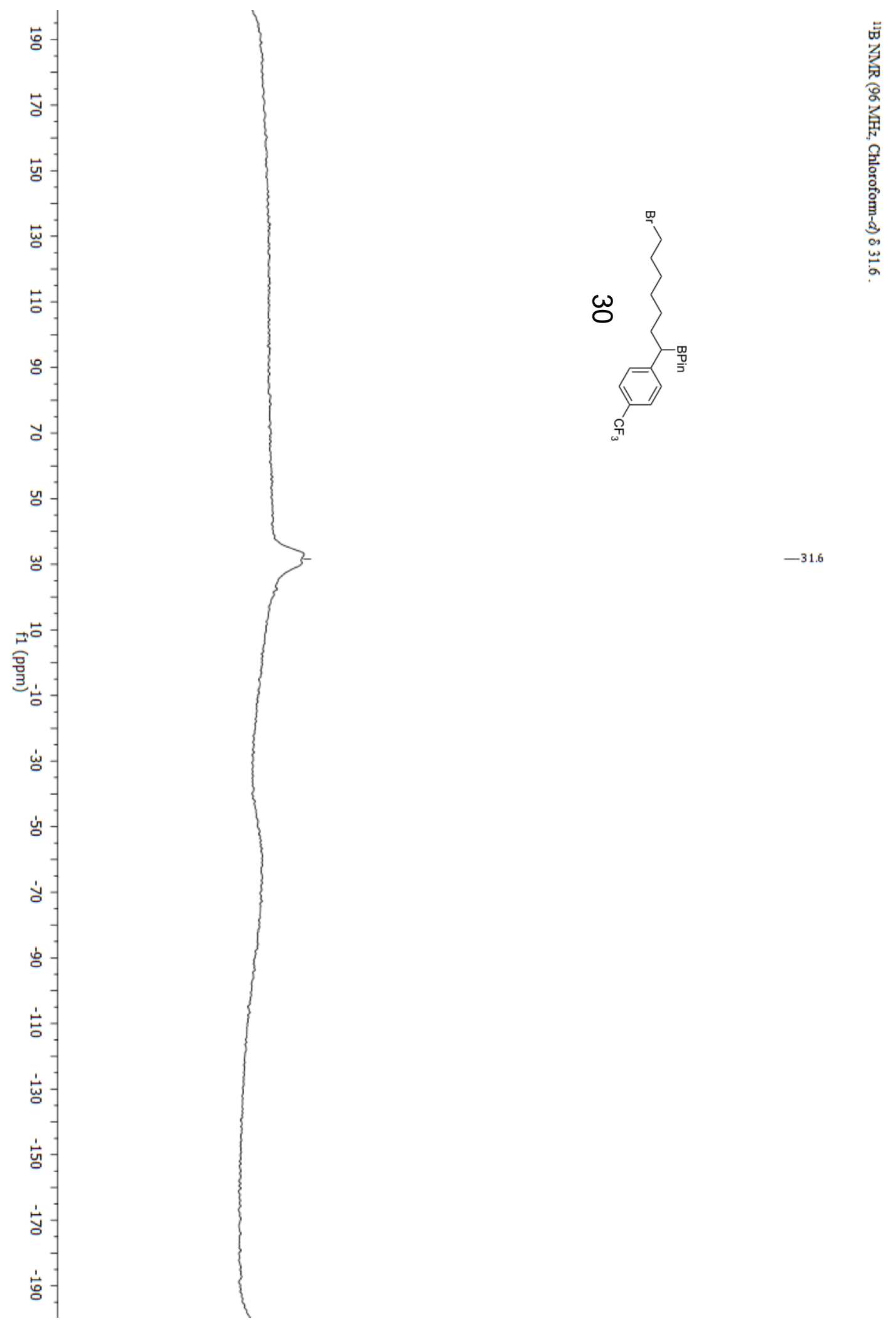


$$
\text { H: }
$$



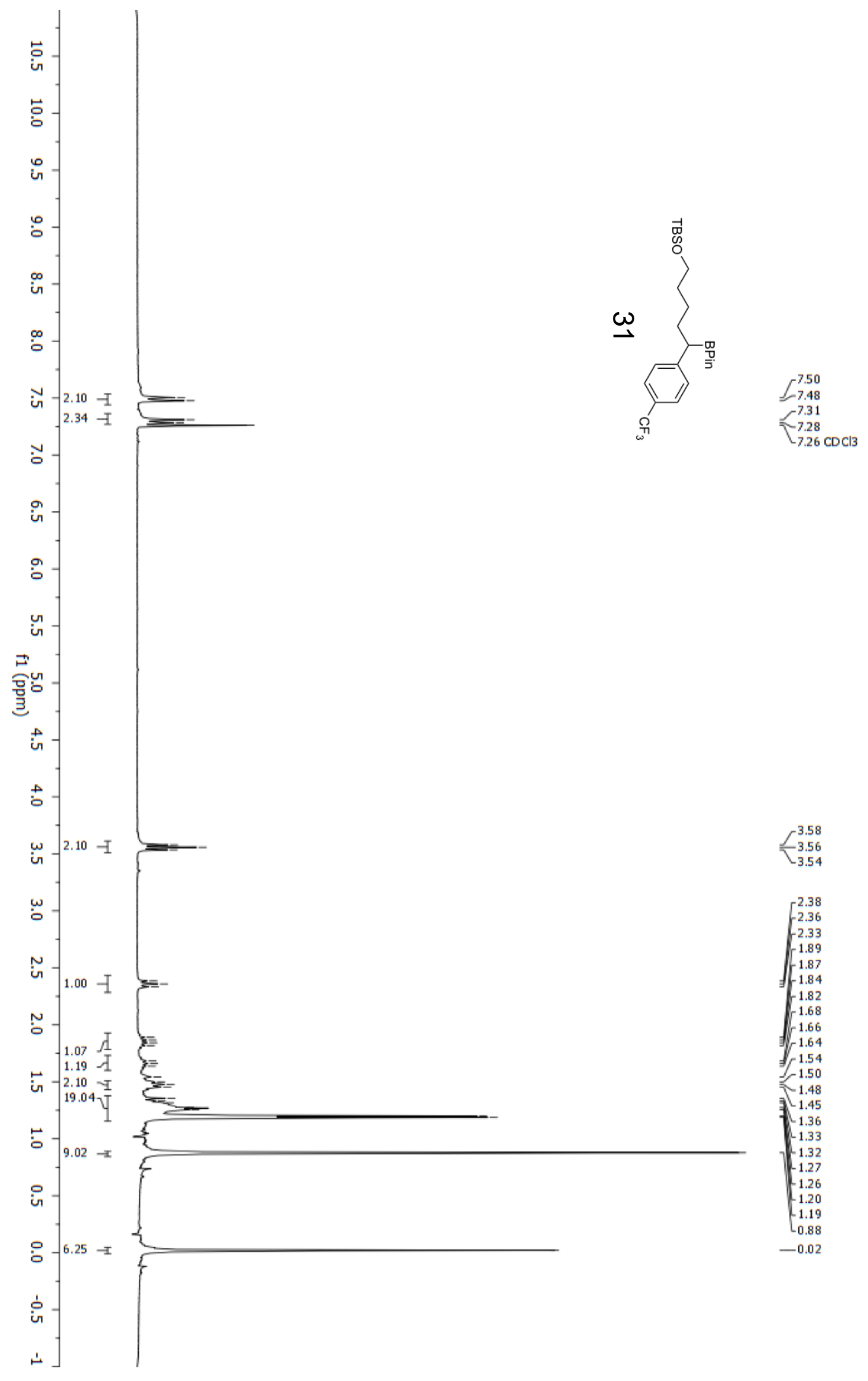

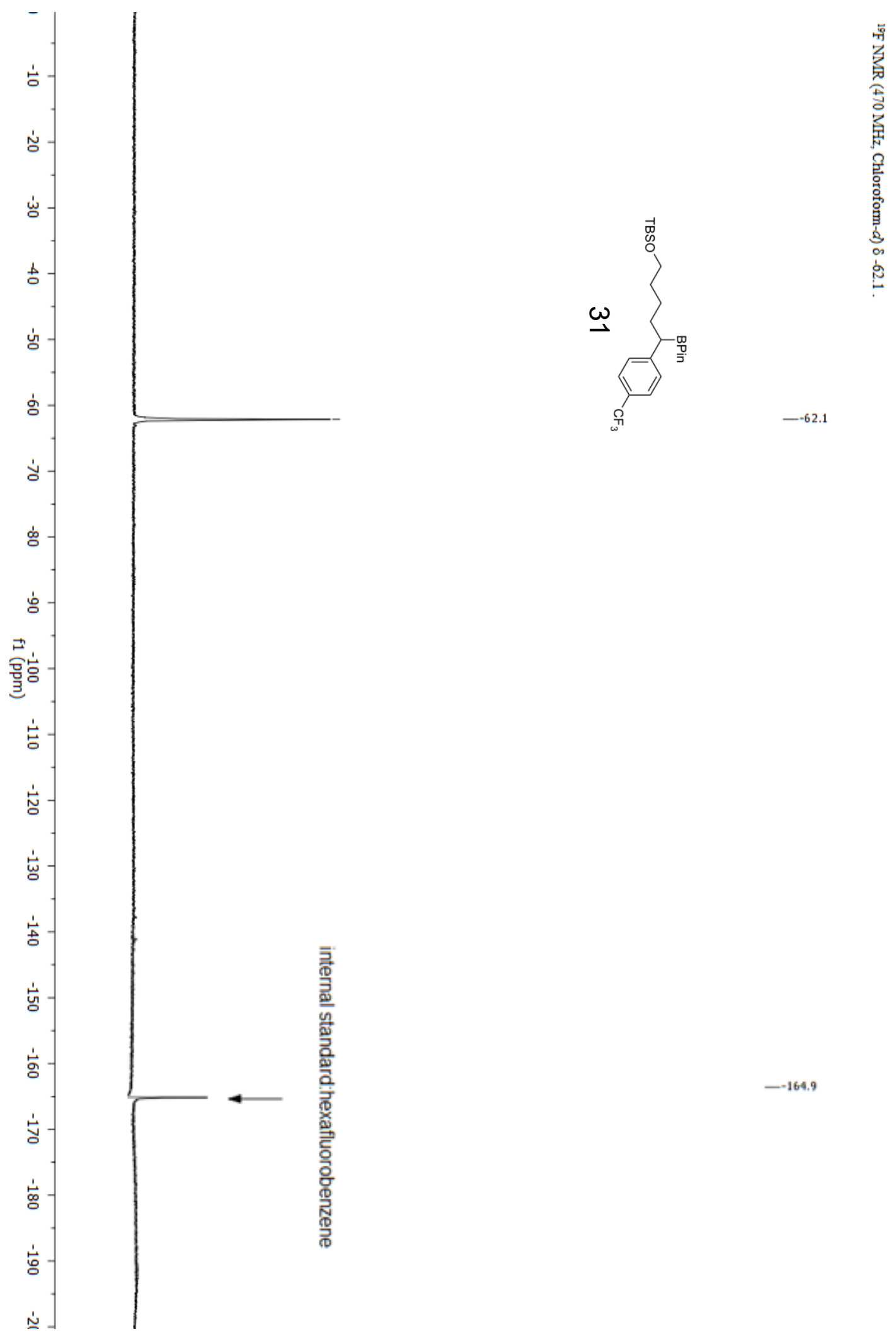

S106 

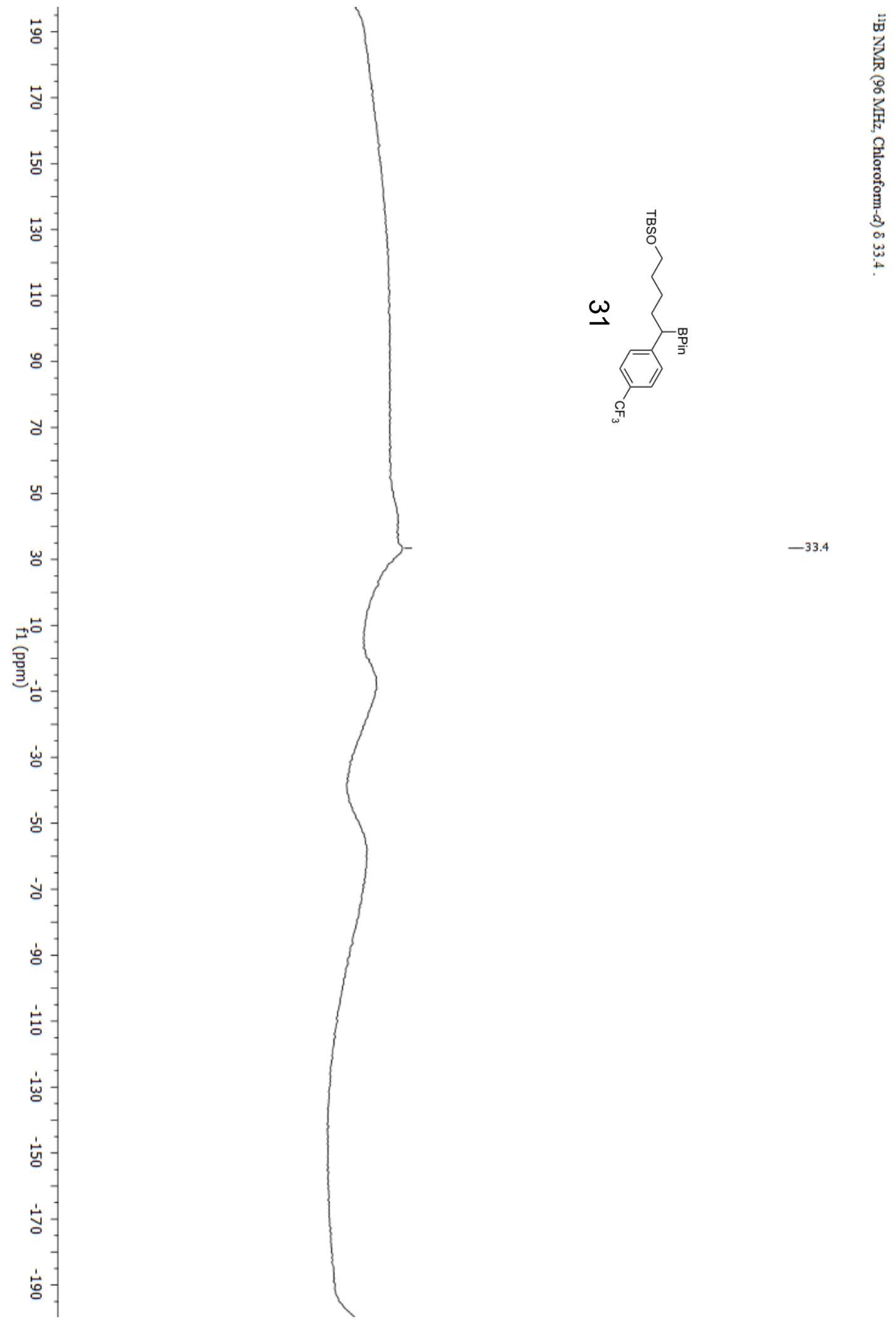

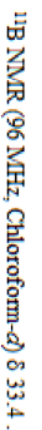

$-33.4$ 


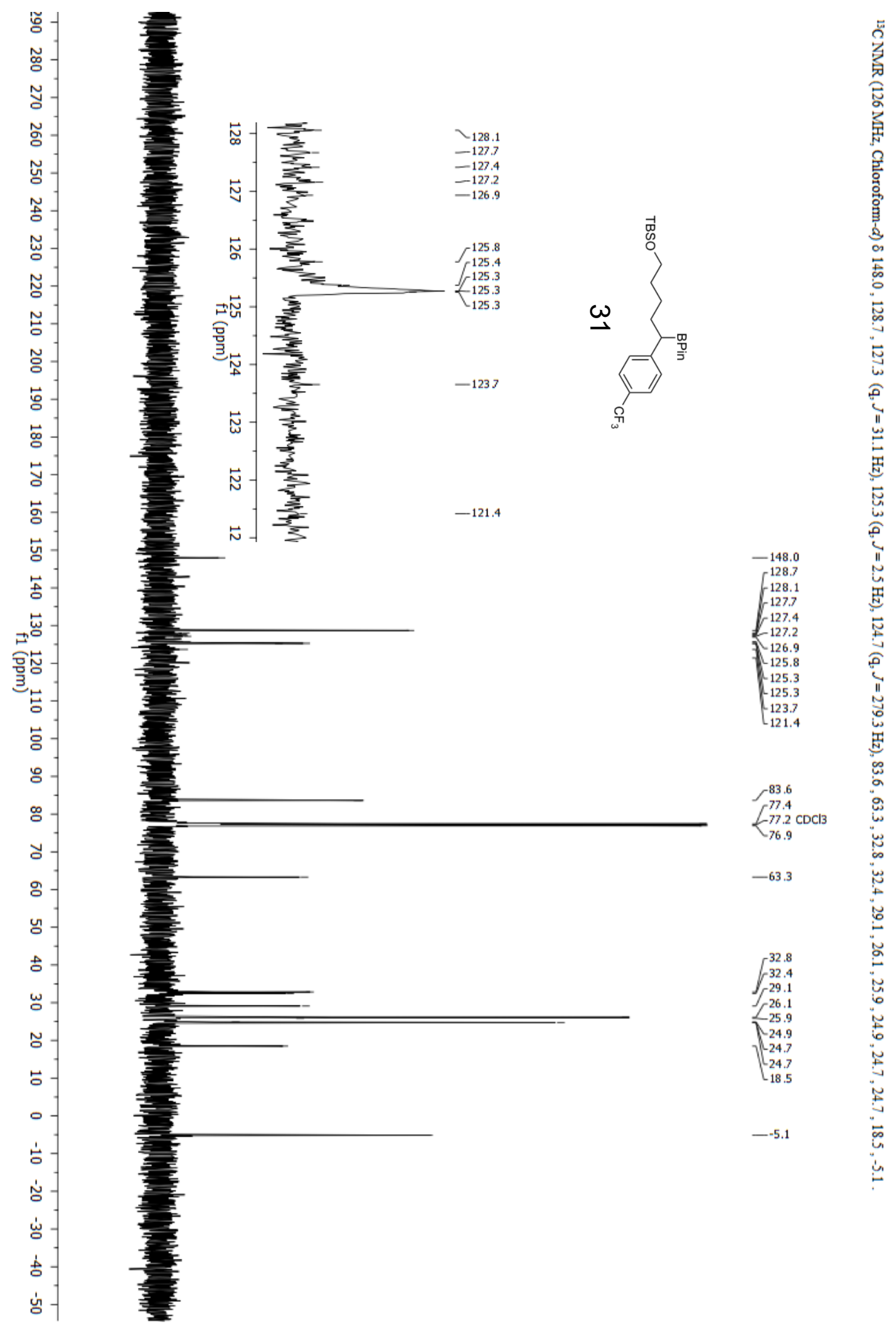



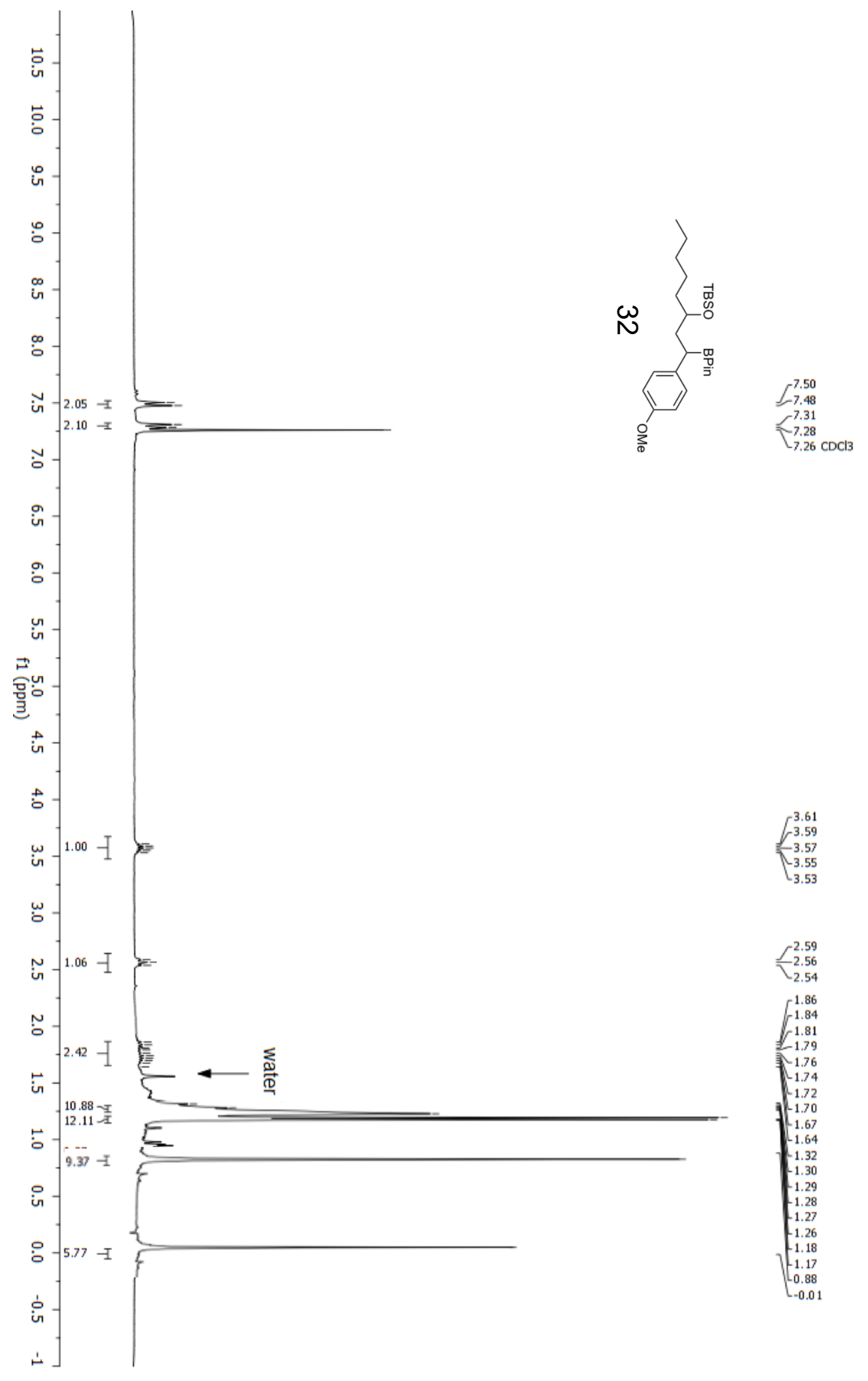

2.59
-2.56

1.86

1.86
1.84
1.81

1.79
-1.76

1.76
1.74

$\Lambda_{1.72}$

- -1.70

$-1.64$

$-1.32$

-1.30
-1.29
-1.28

-1.28
-1.27

-1.27
-1.18

$\left\{\begin{array}{r}-1.18 \\ -1.17 \\ 0.88\end{array}\right.$

0.88
-0.01

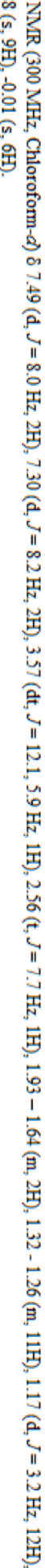



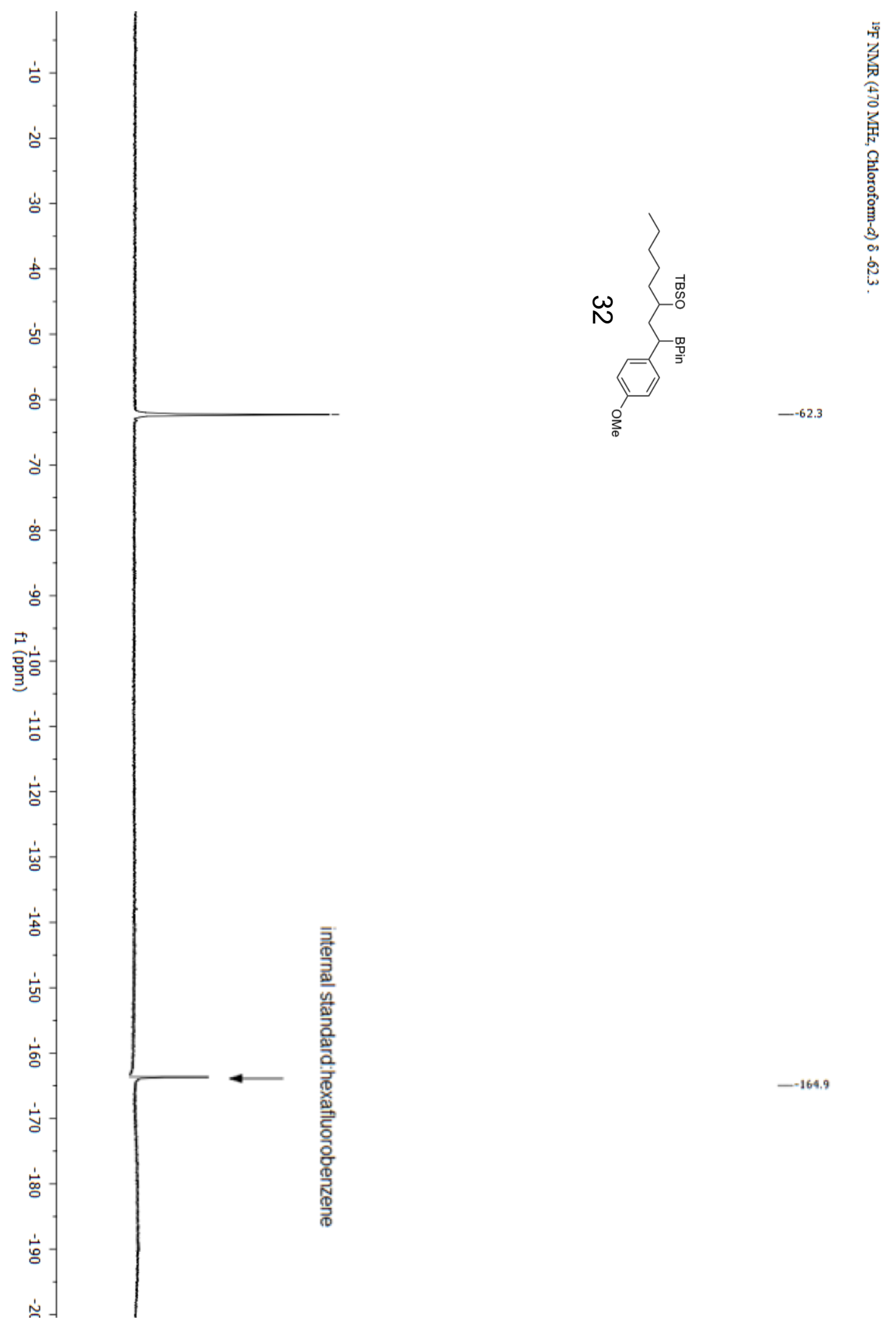

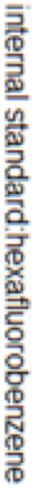
$--164.9$ 

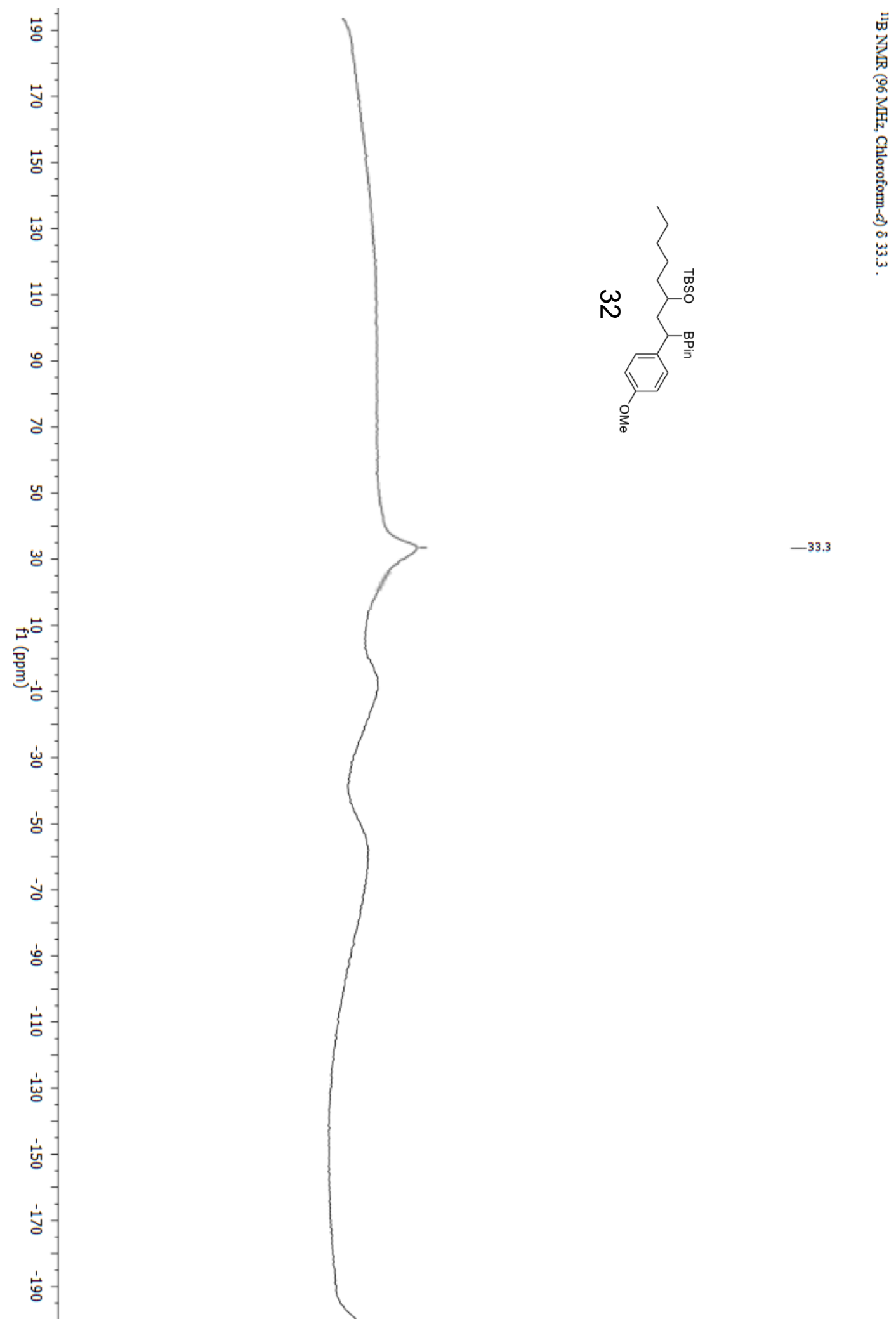

$-33.3$ 


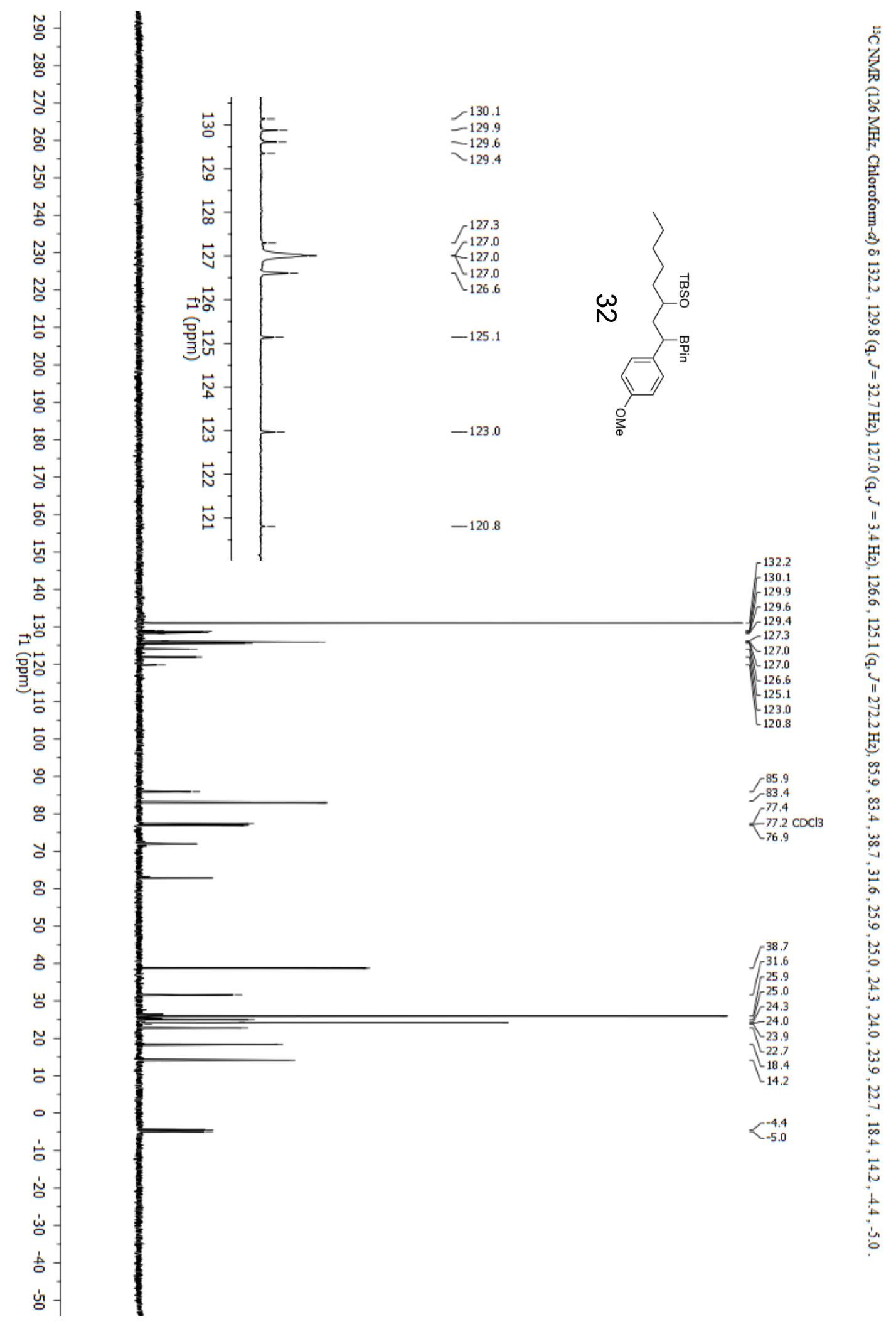




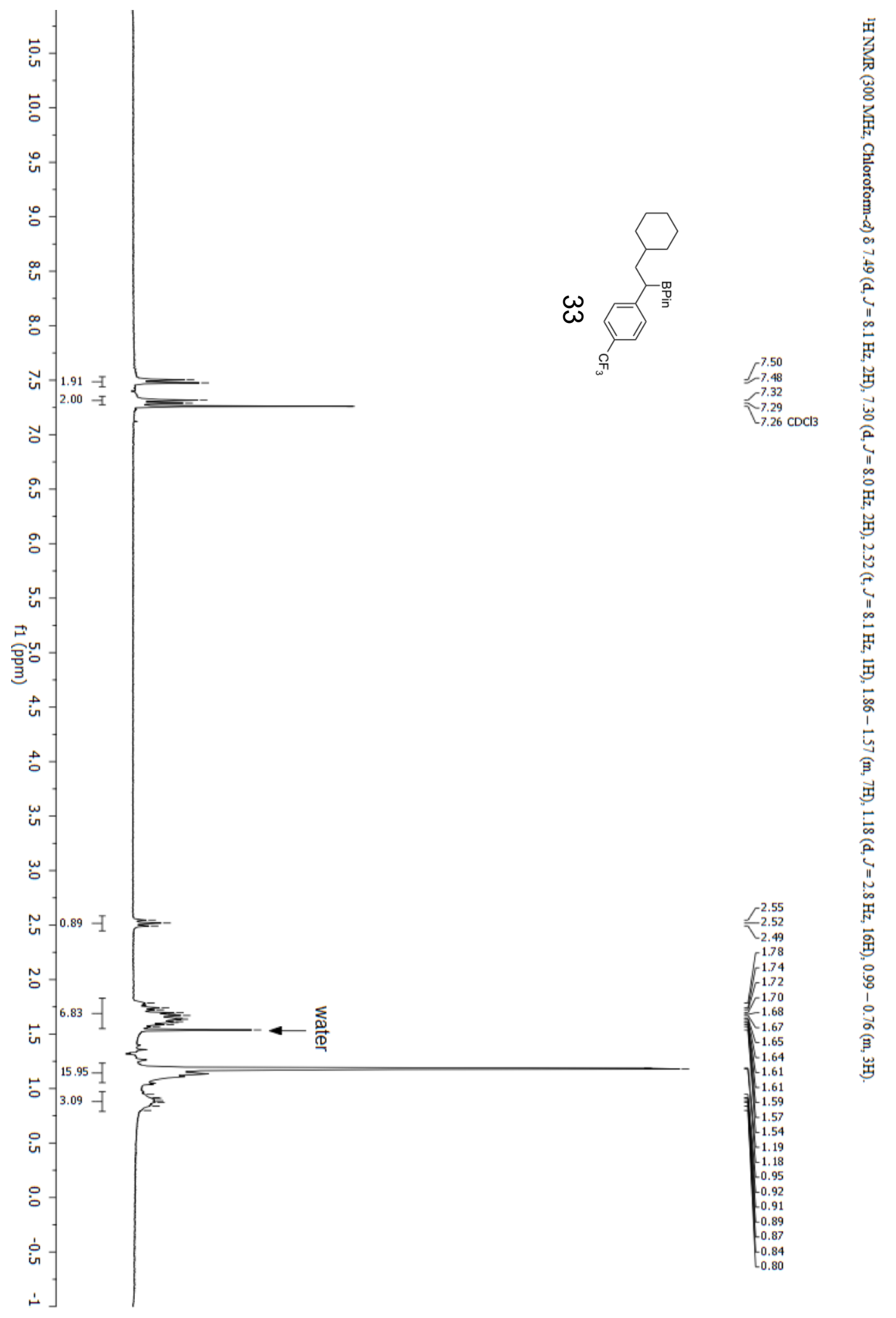



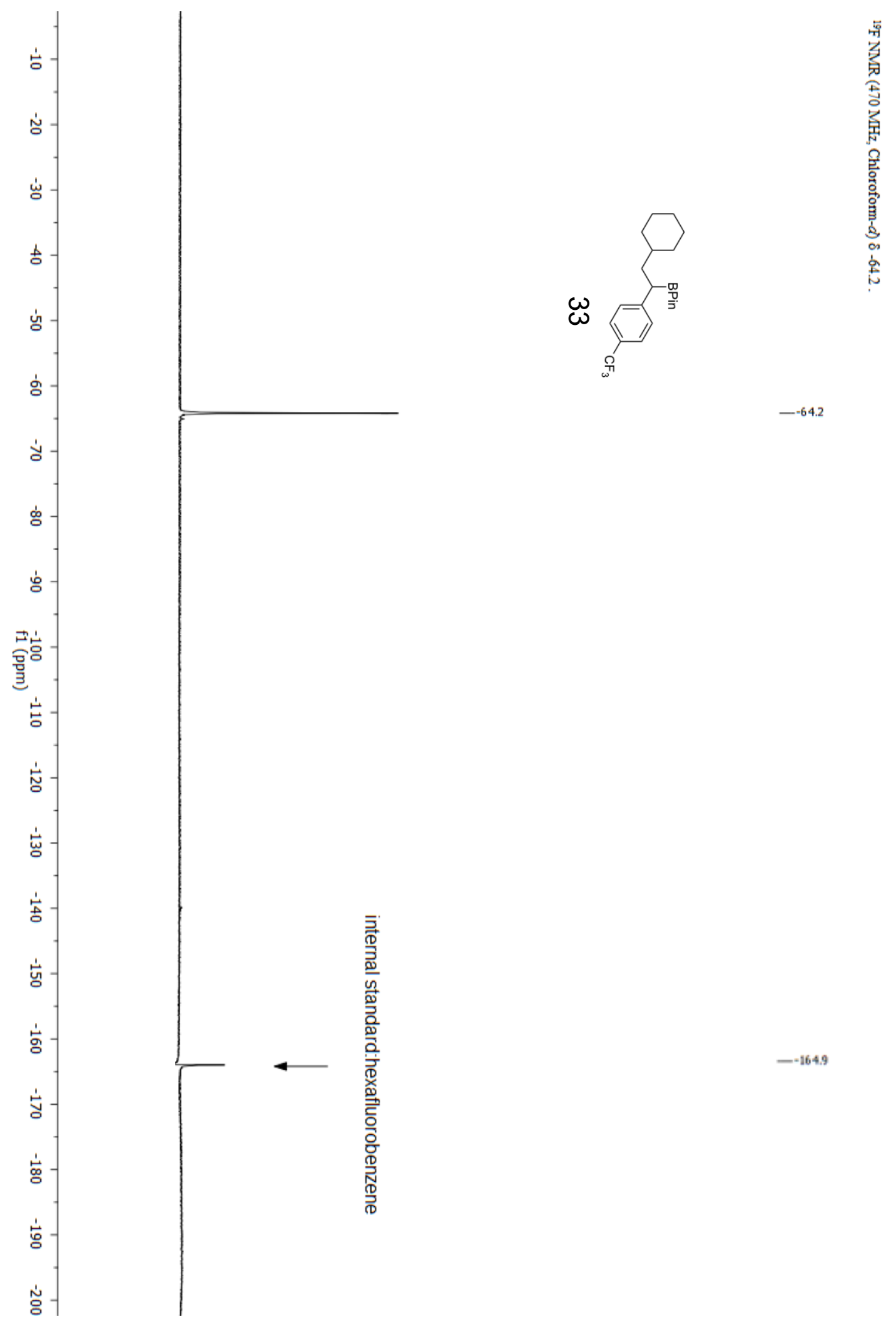

$-64.2$

$--164.9$ 

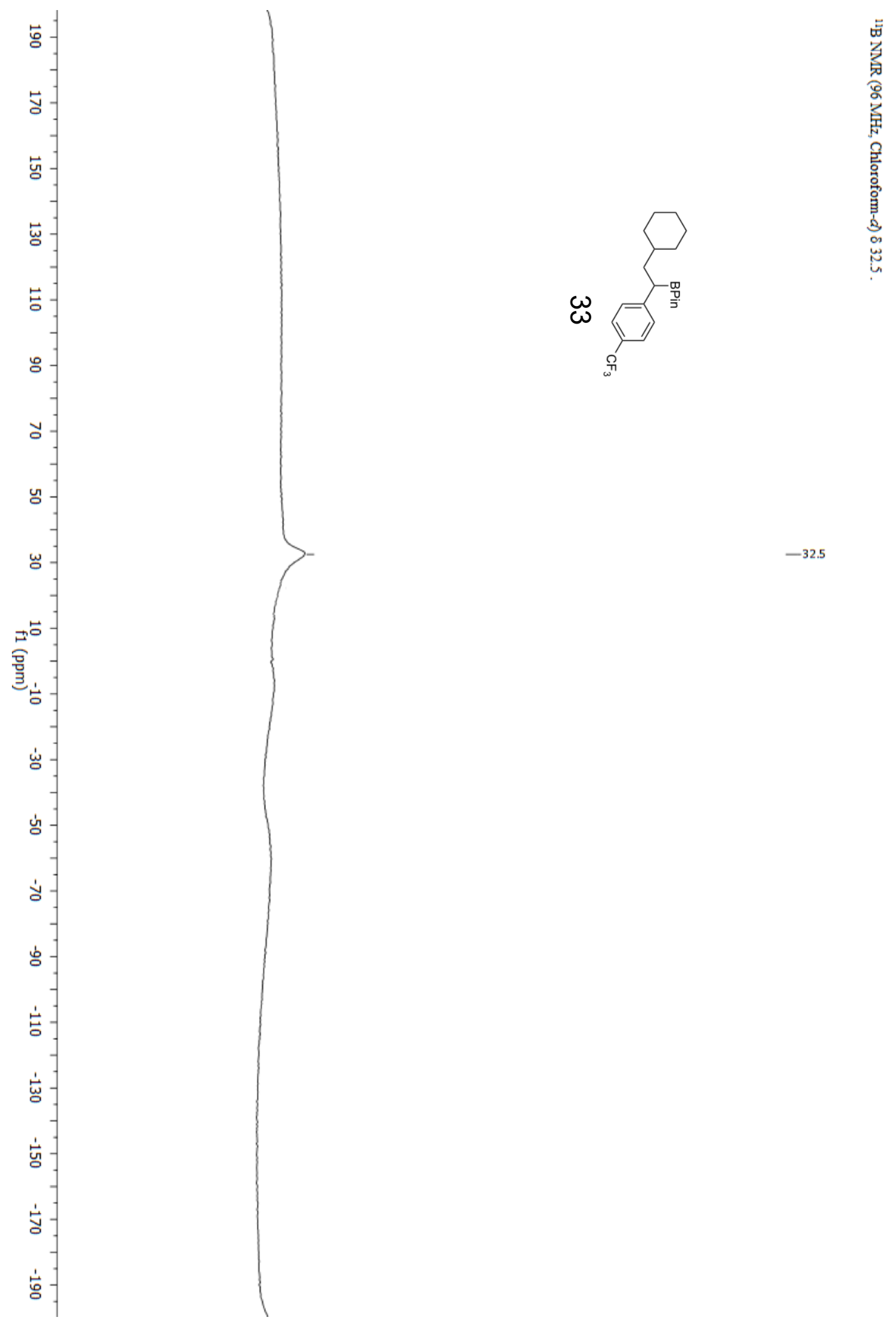

$-32.5$ 


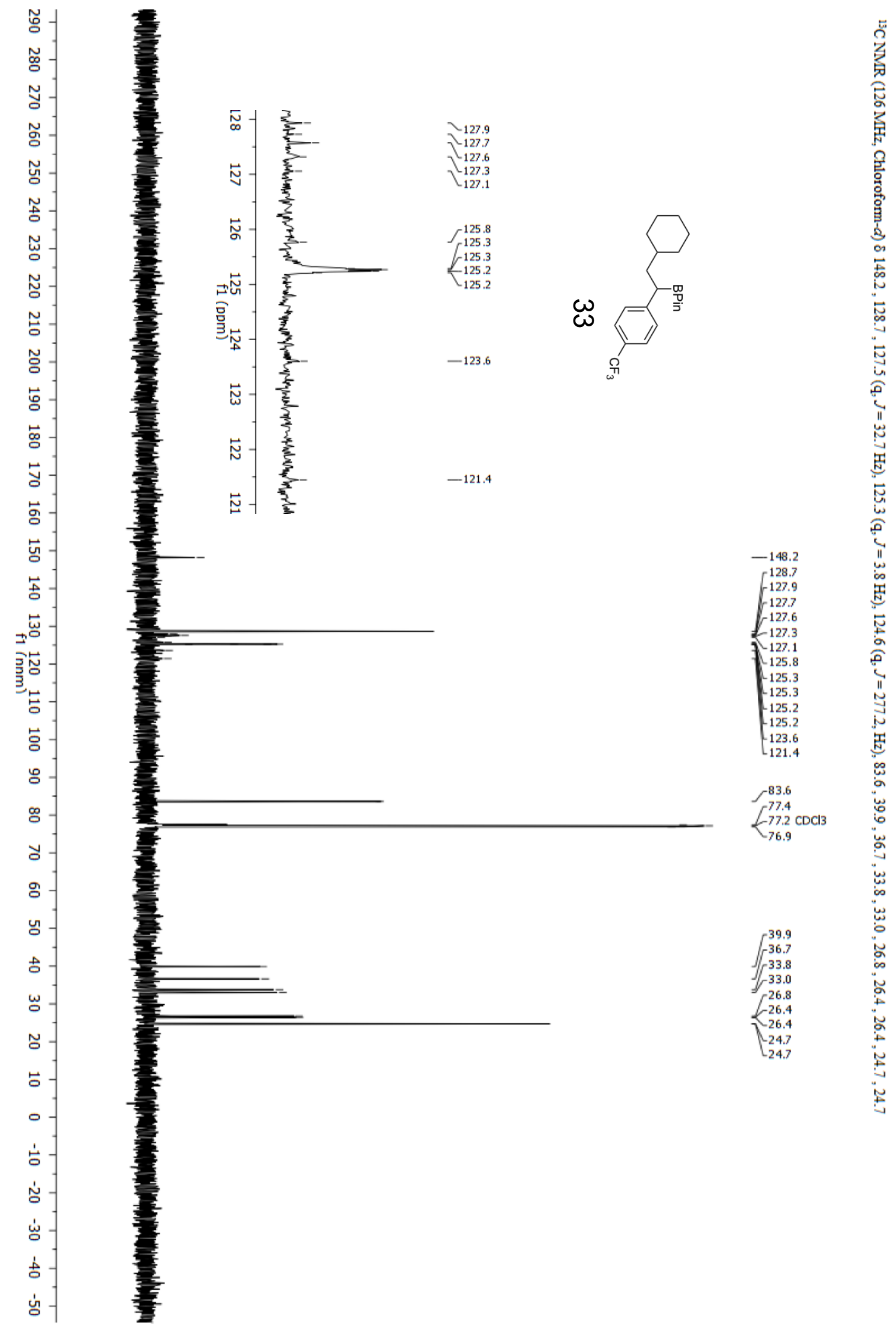




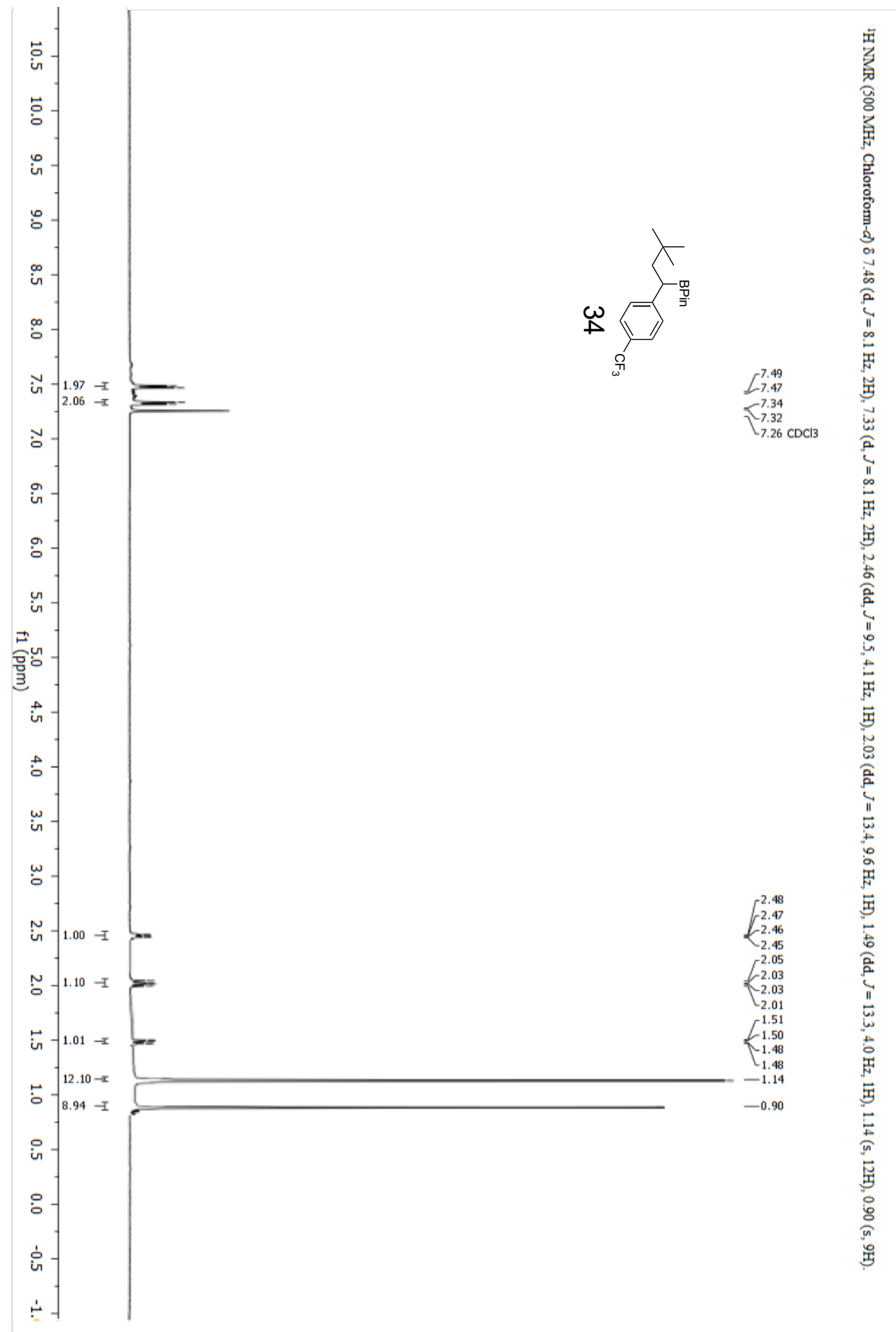



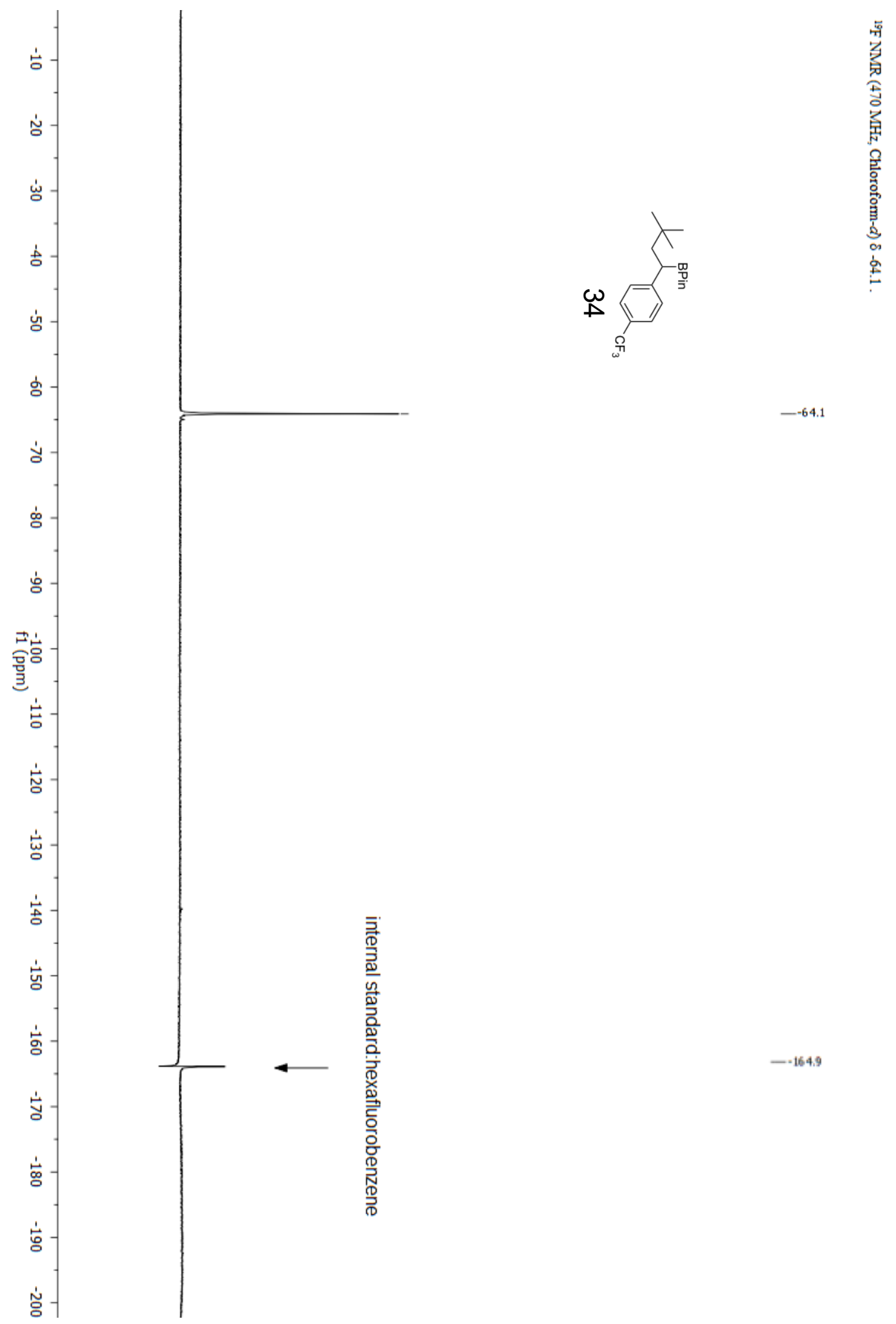

$-64.1$ 

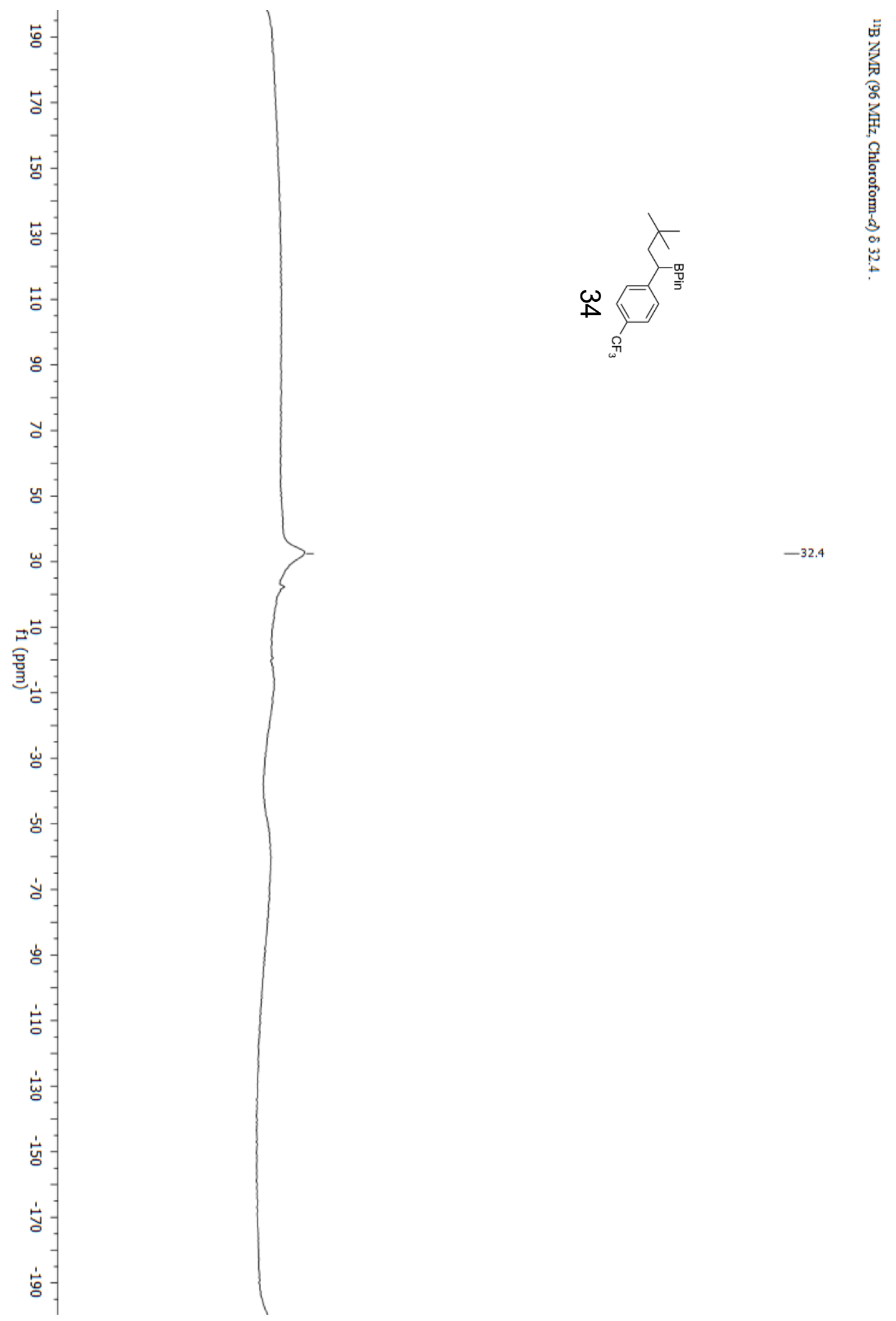


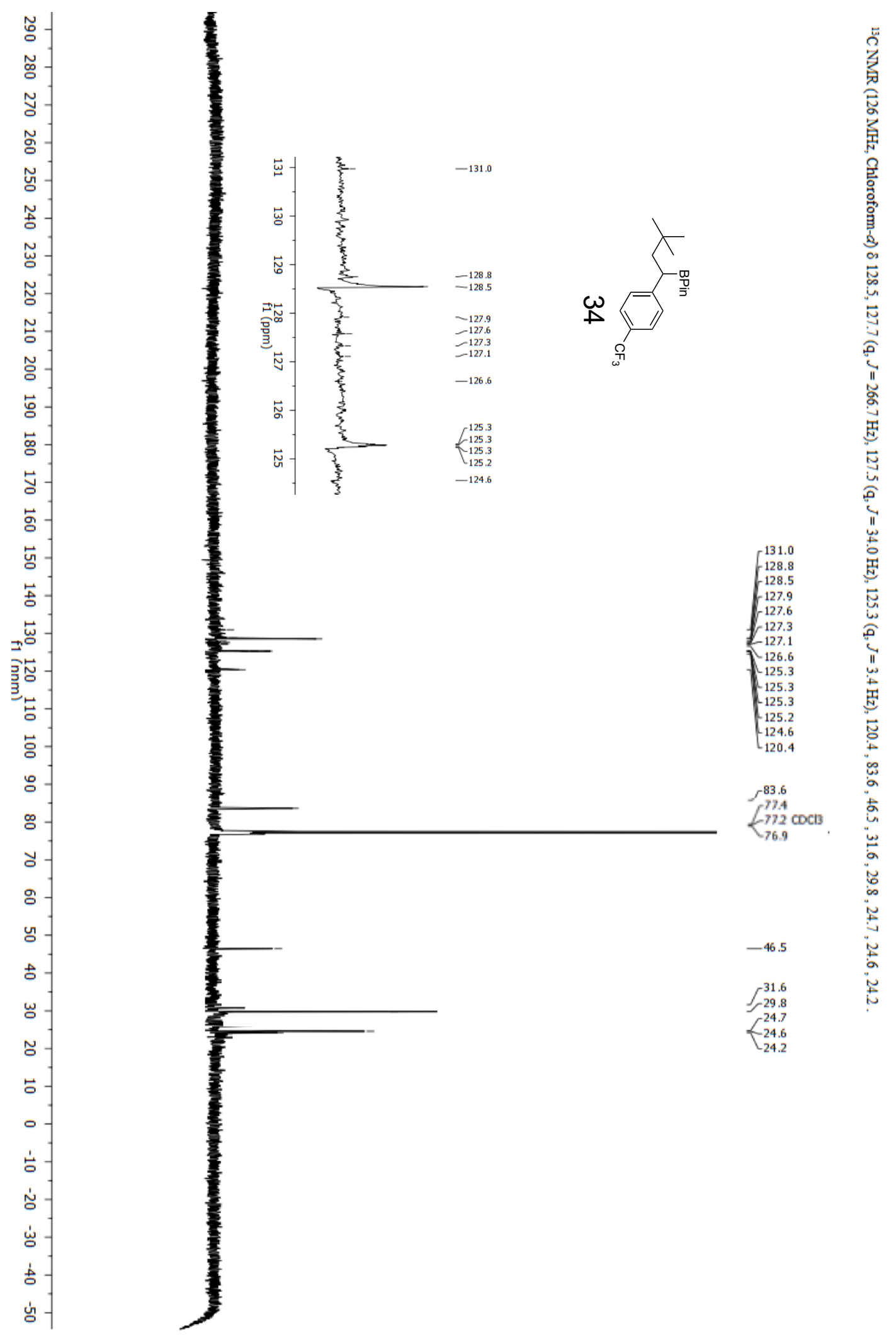




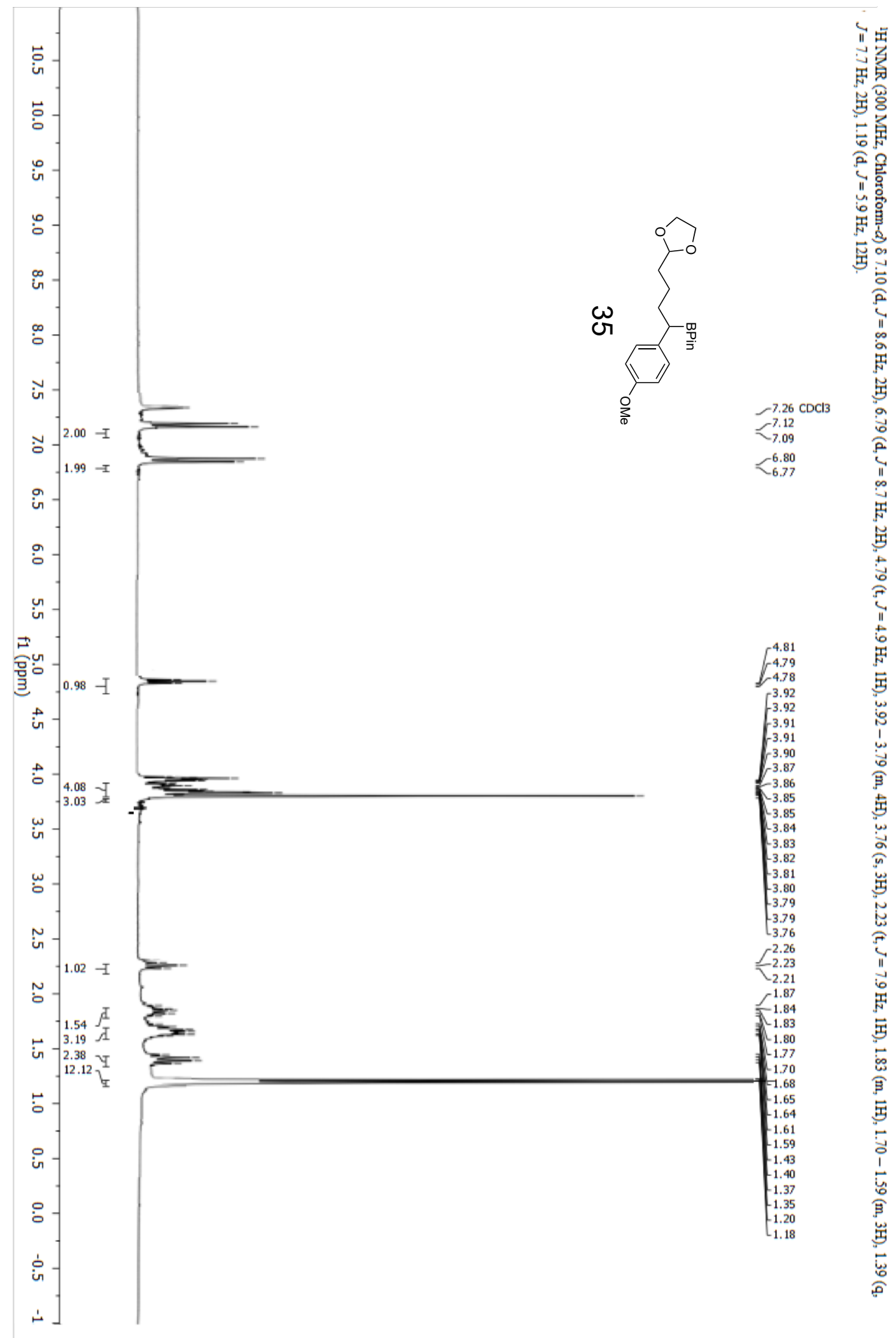



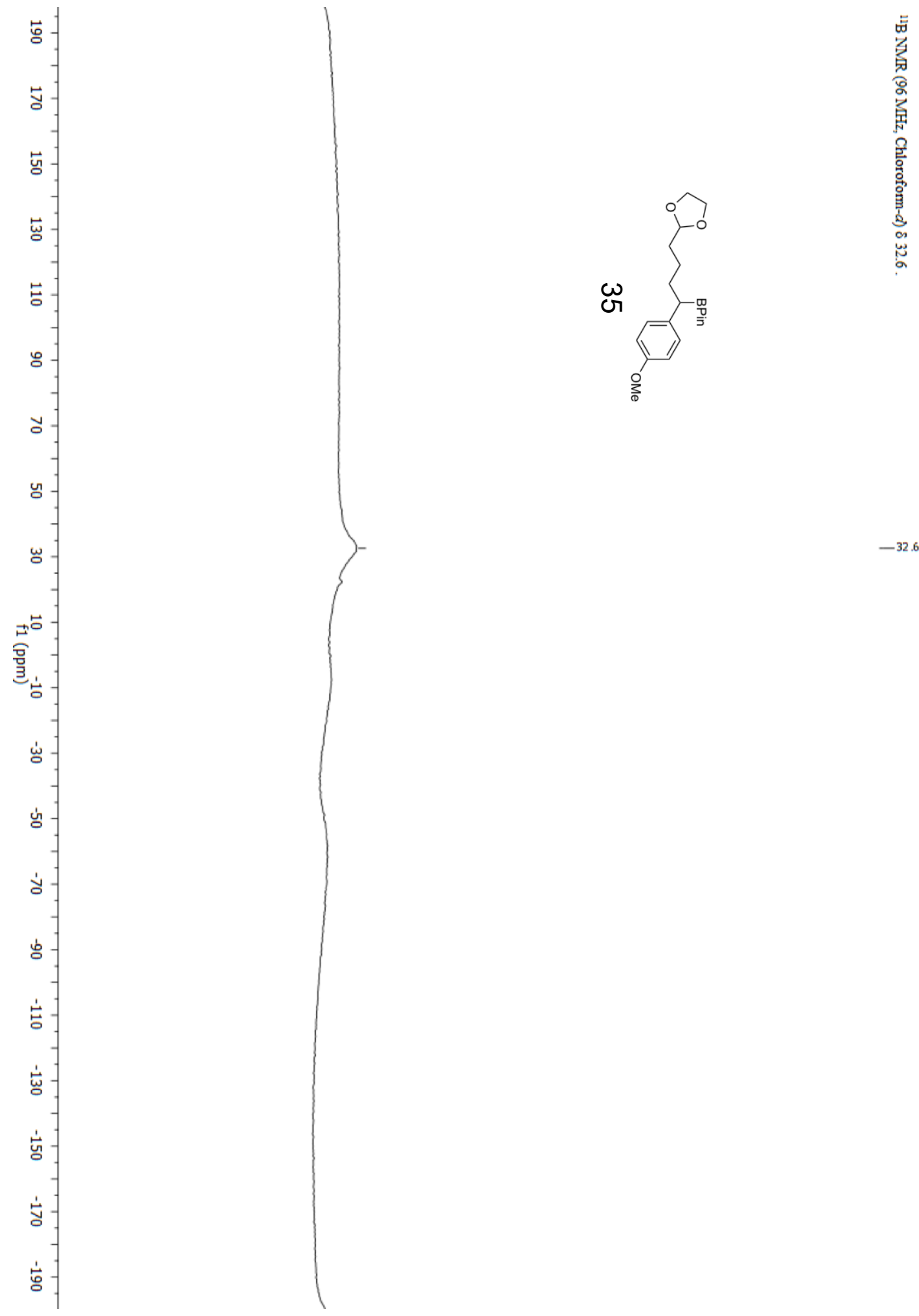

w

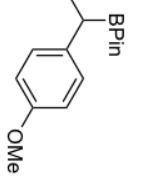

$-32.6$ 


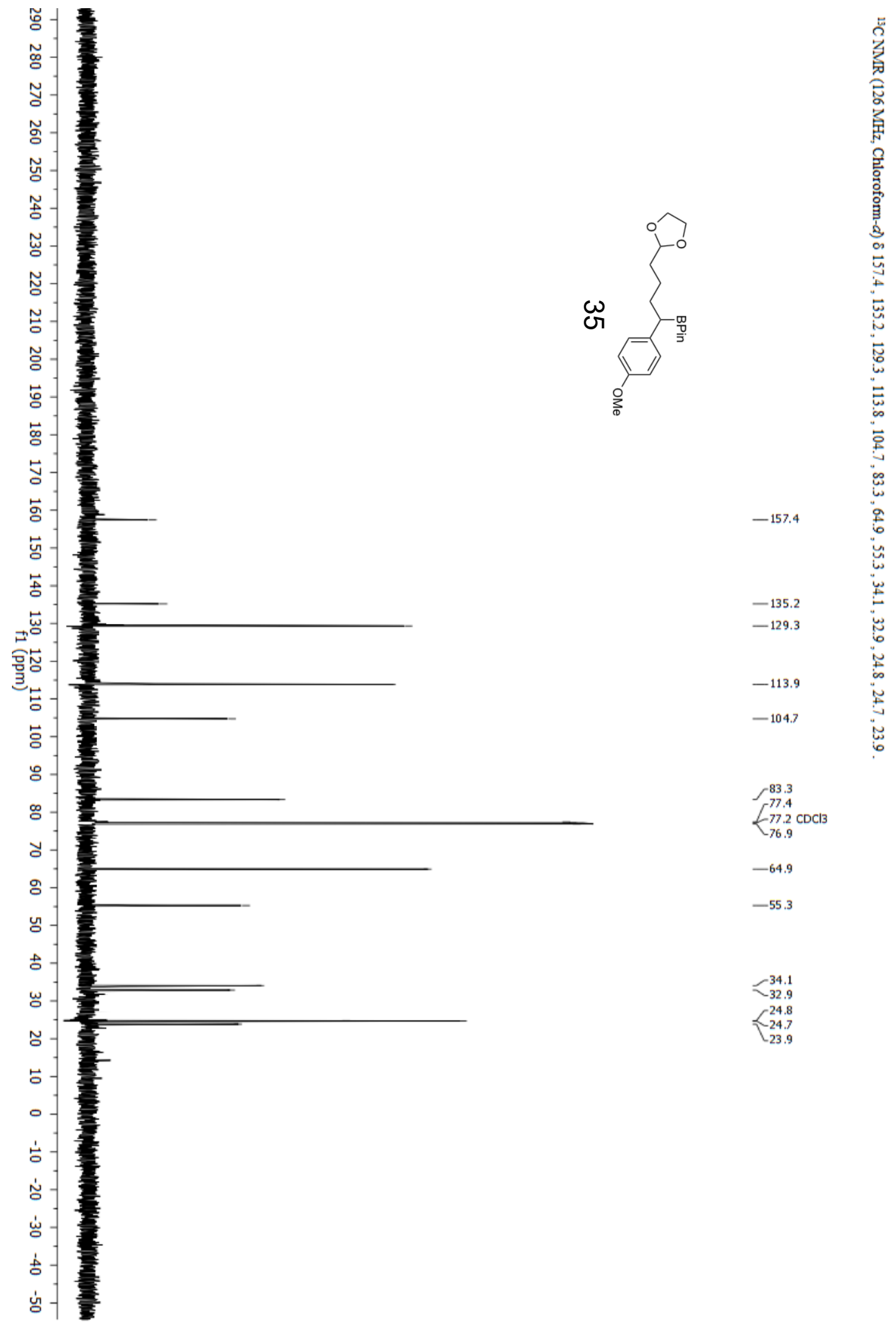




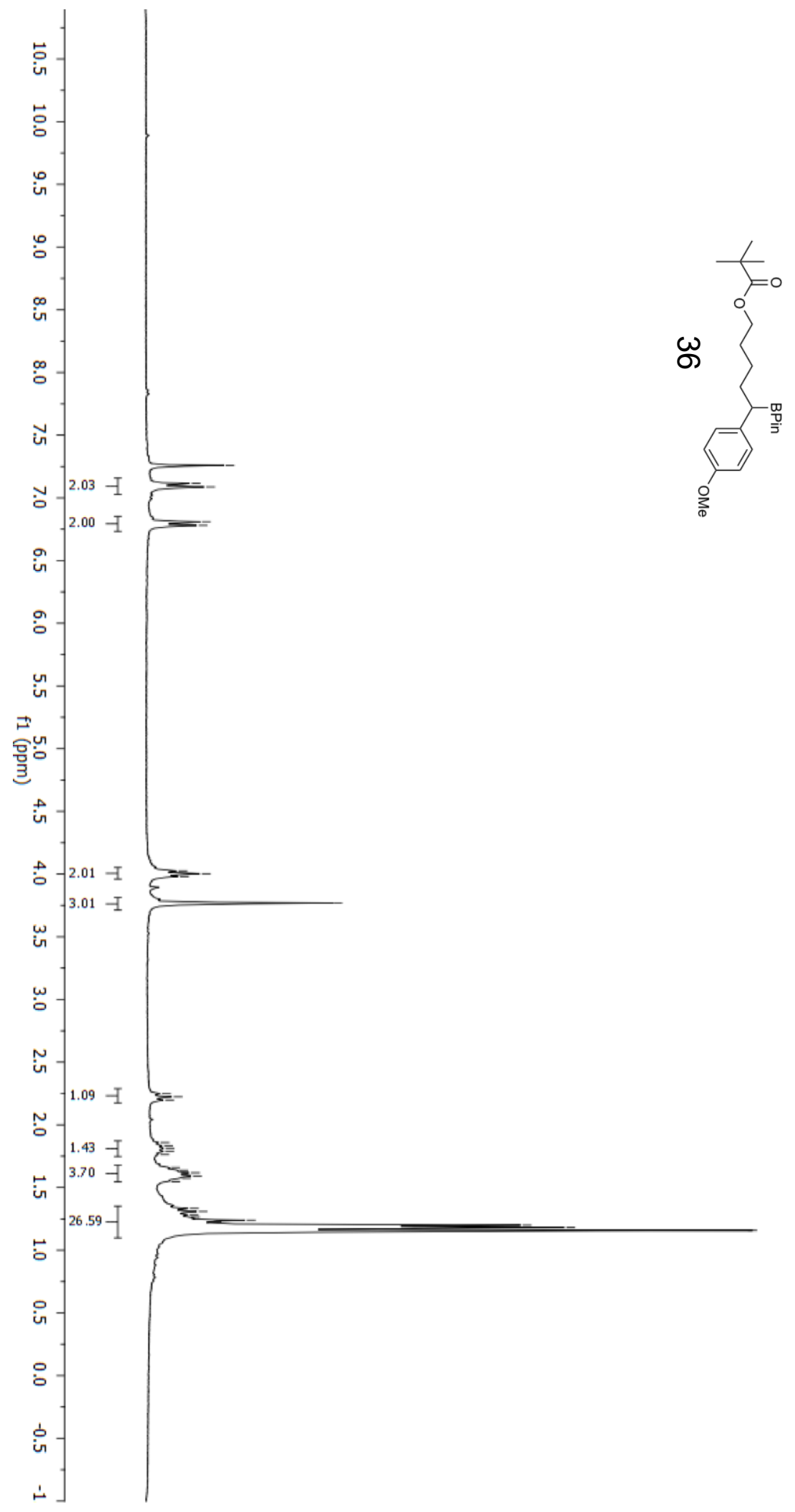



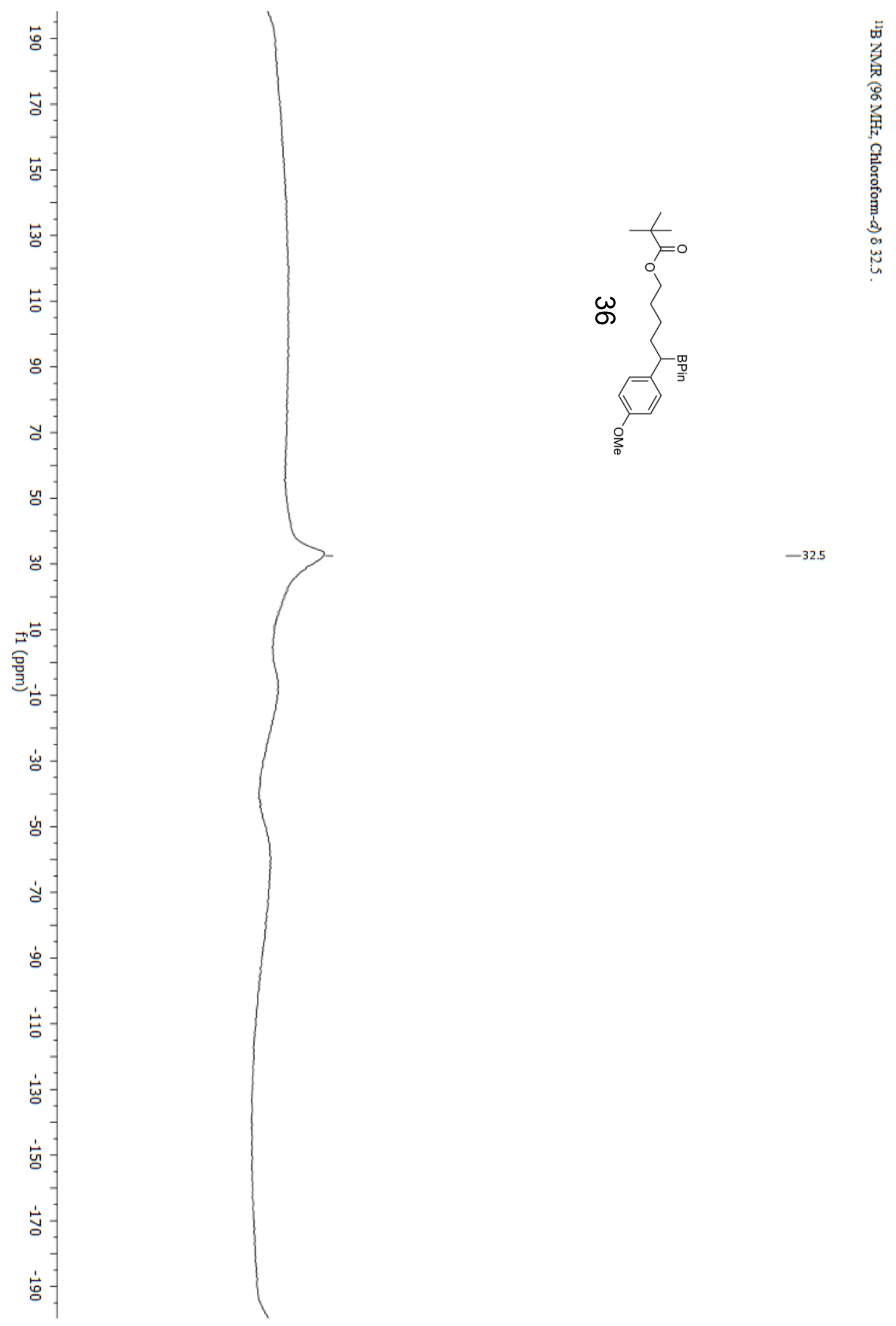

$-32.5$ 


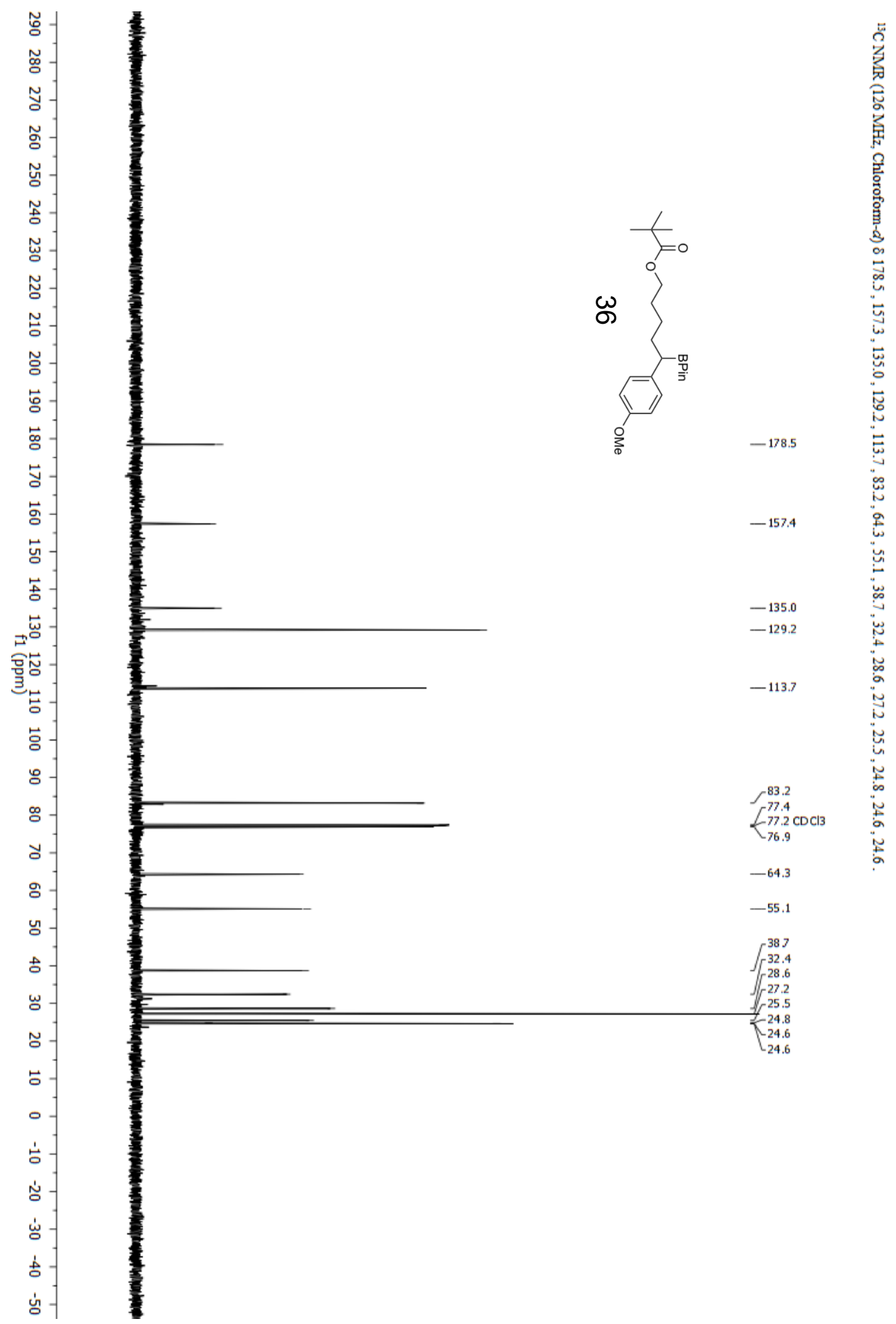




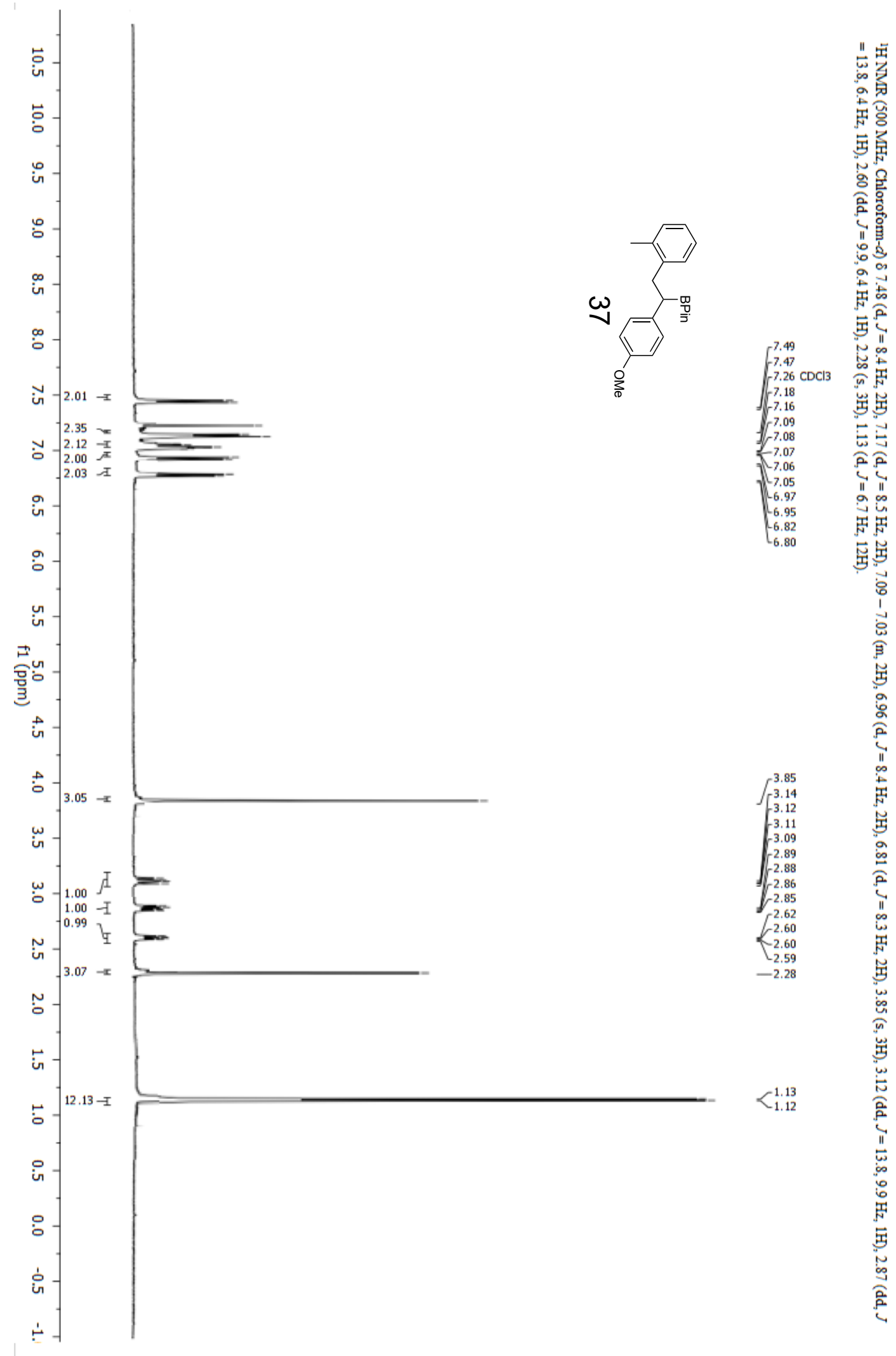



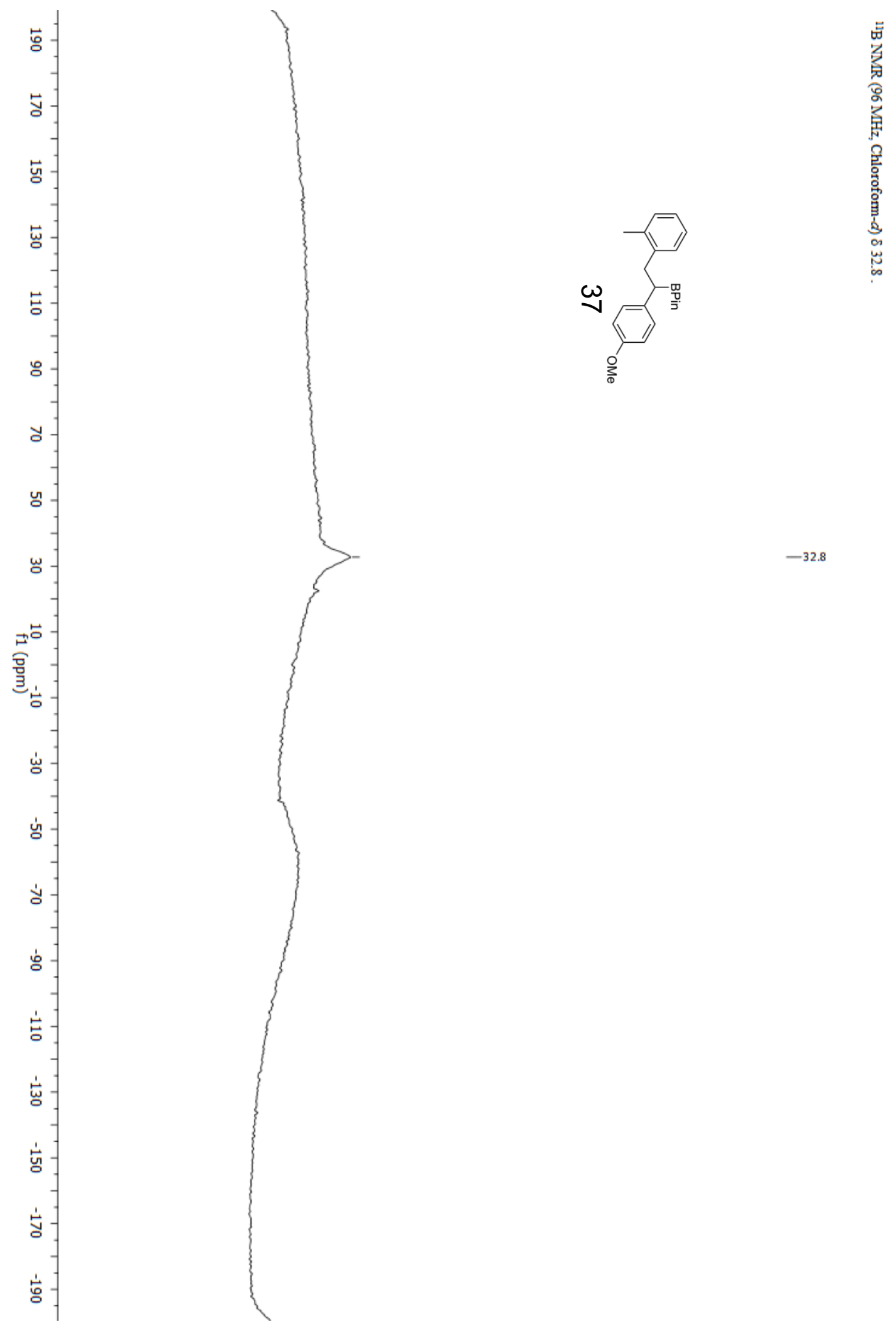

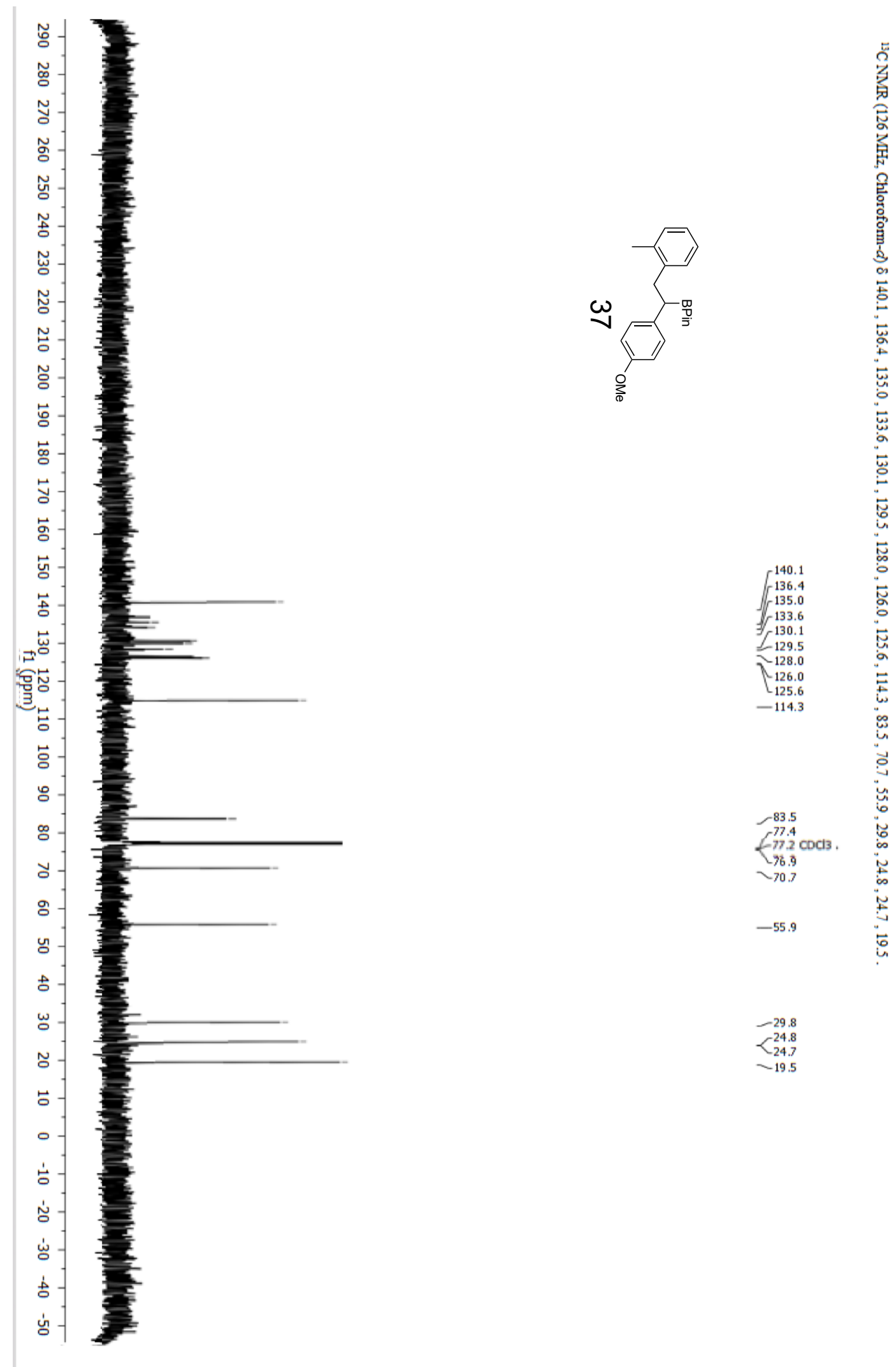


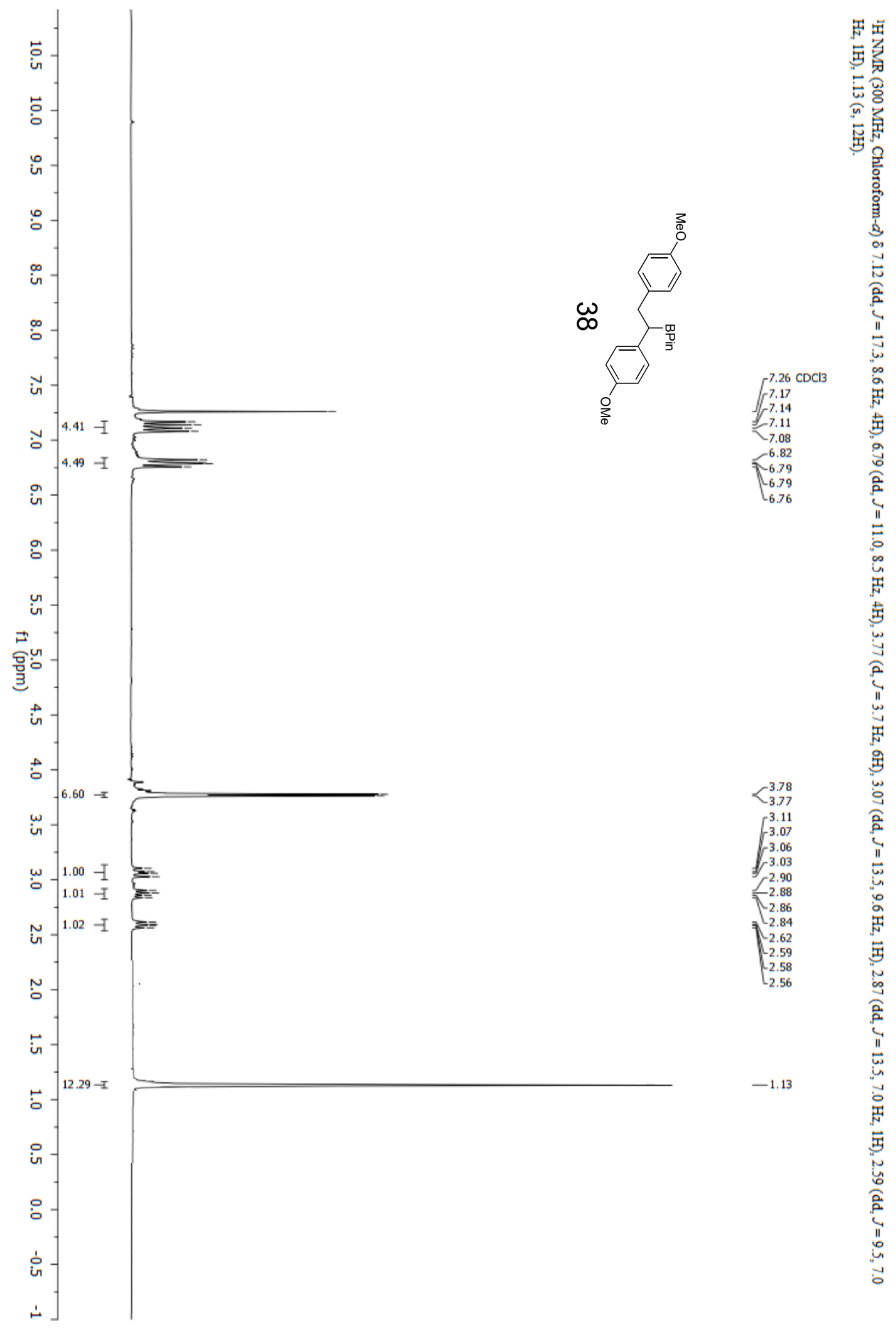



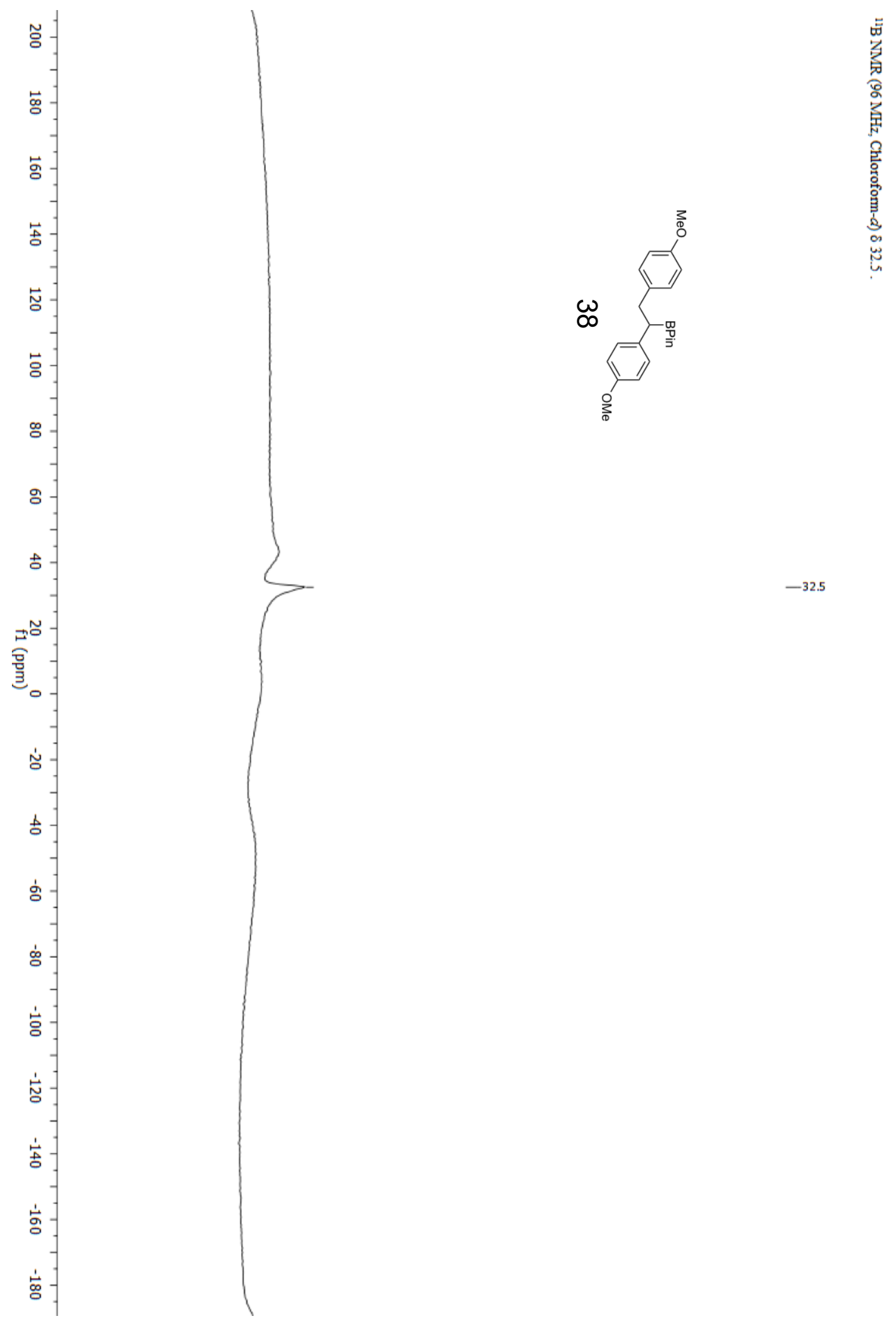

$-32.5$ 


$$
\mid
$$




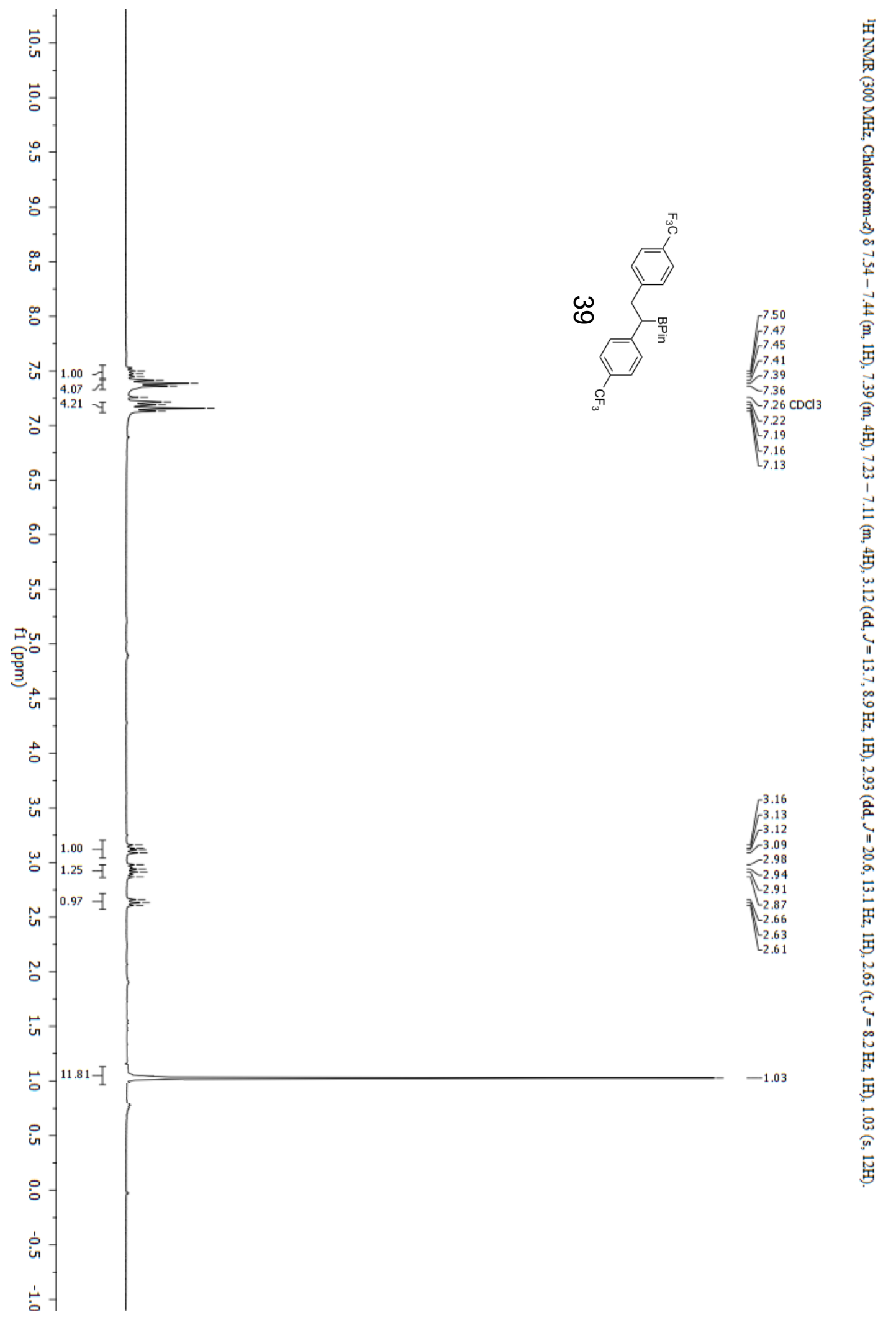




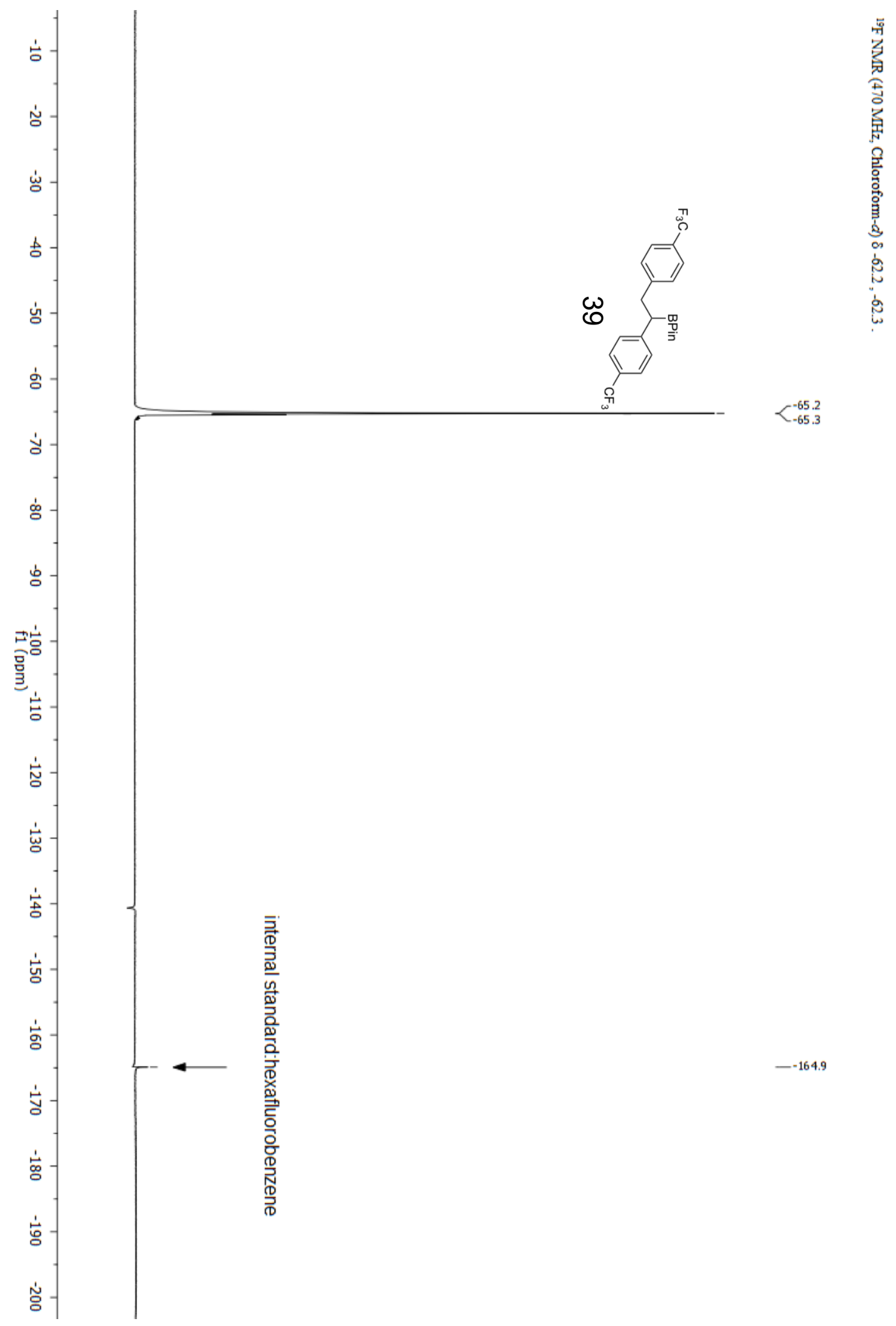




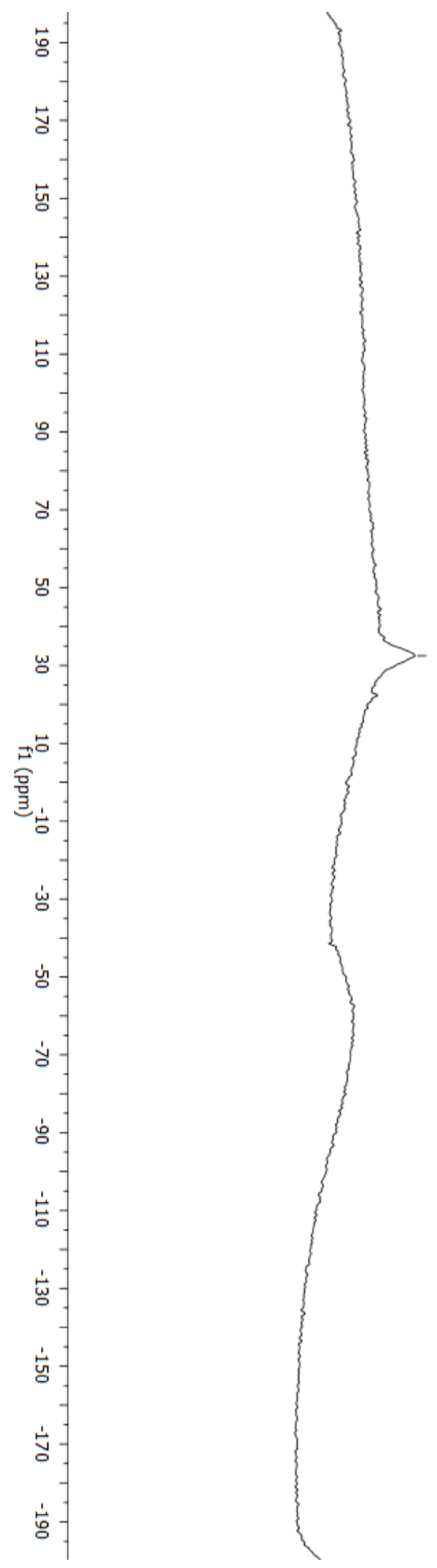




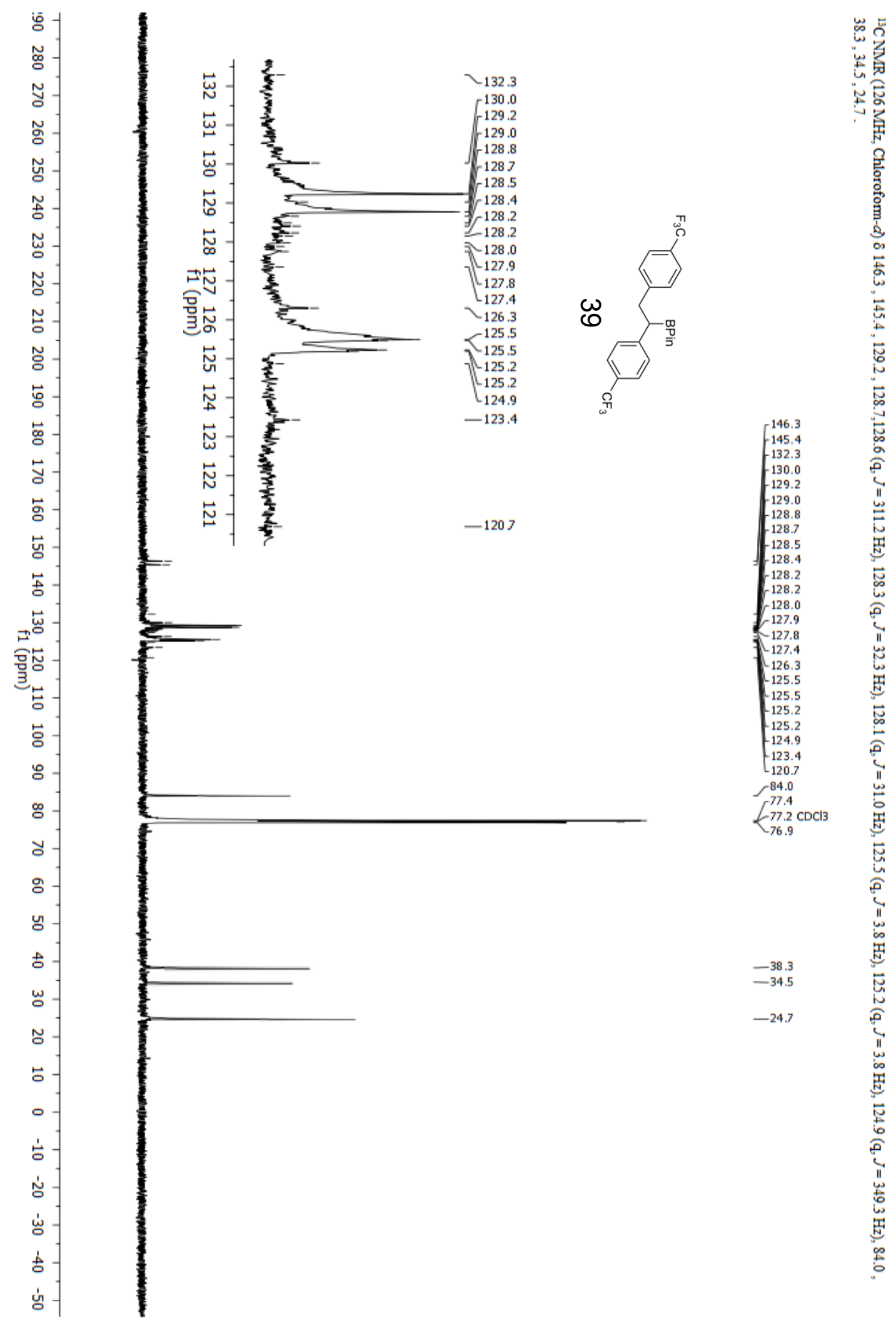




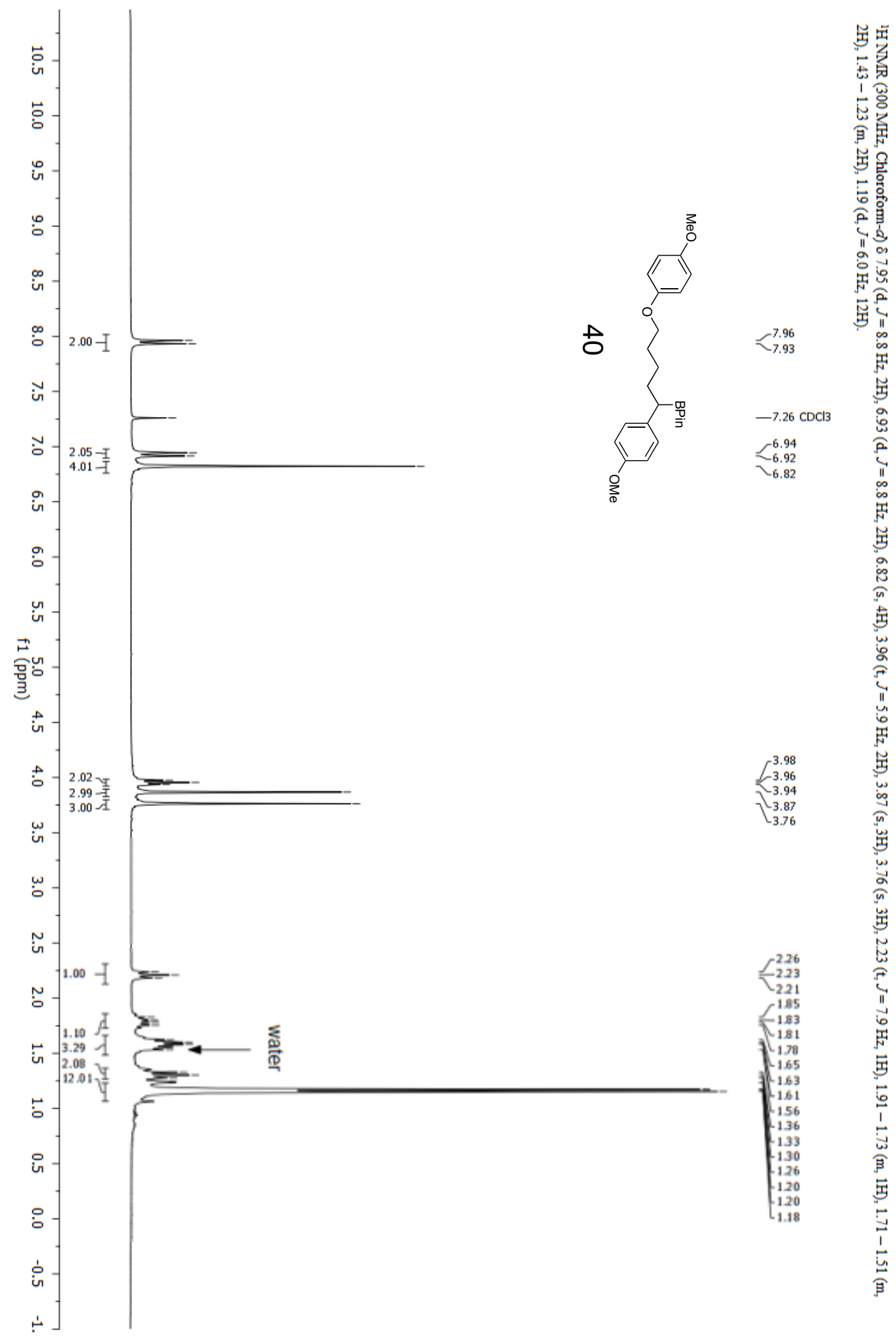



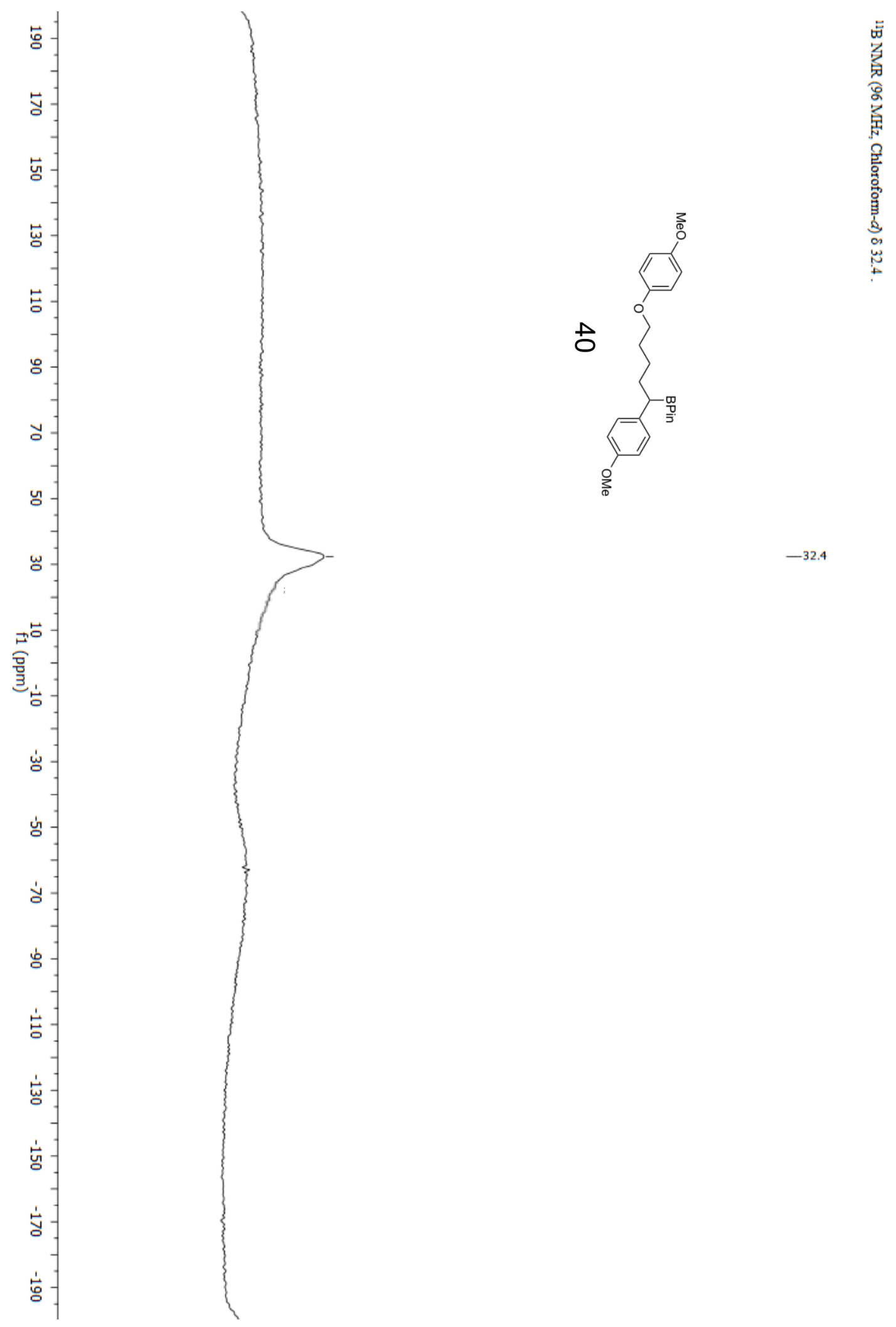

$-32.4$ 


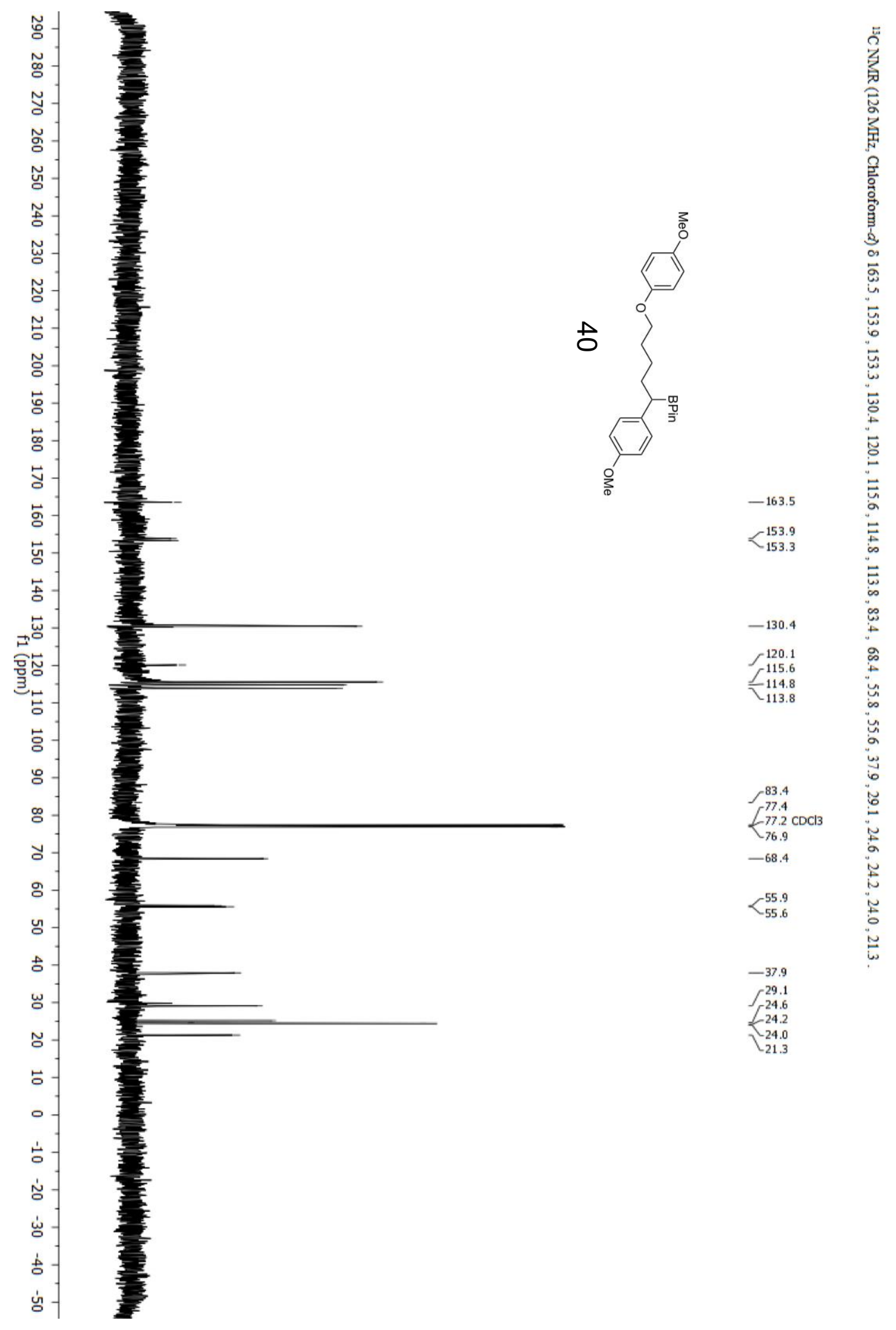




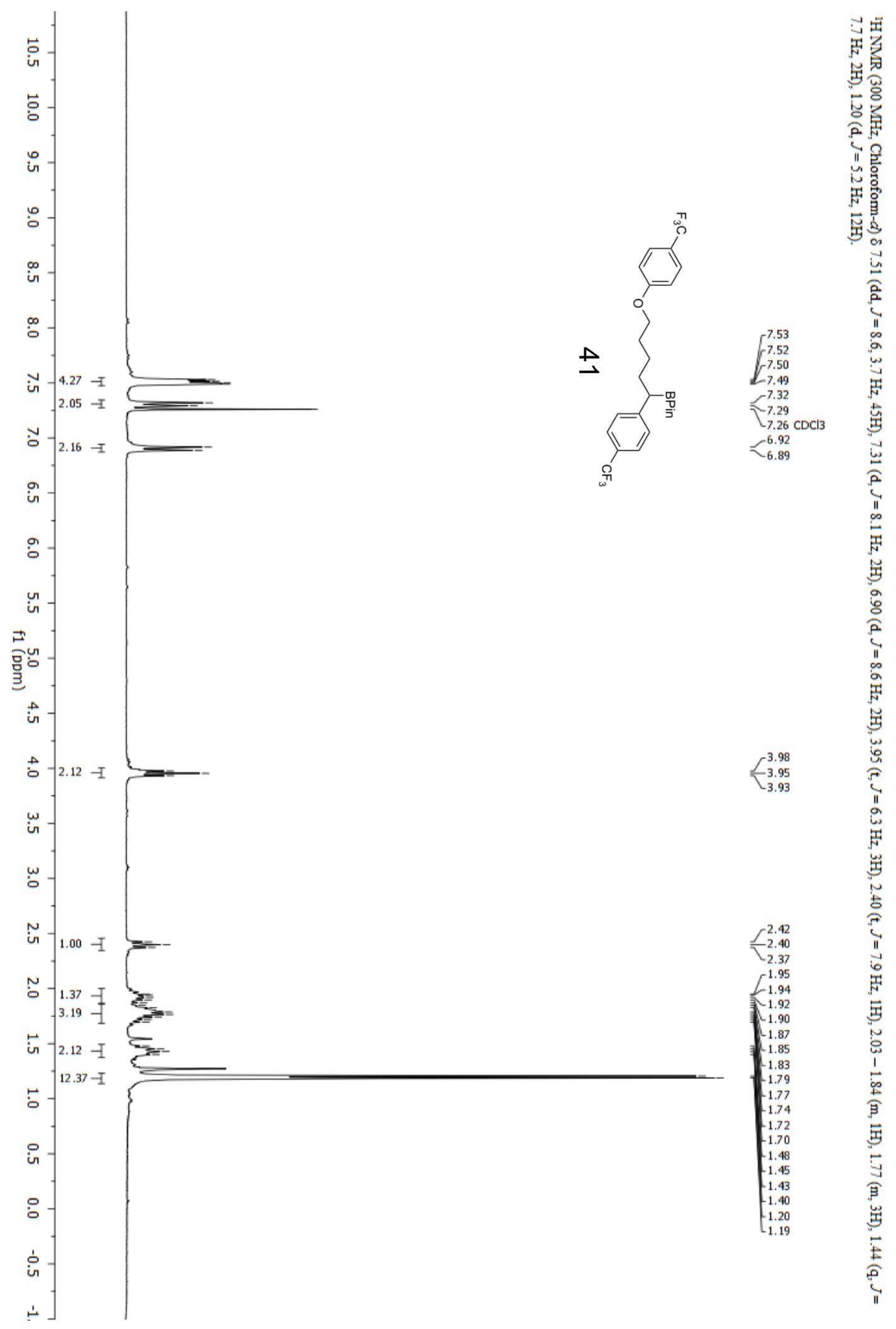



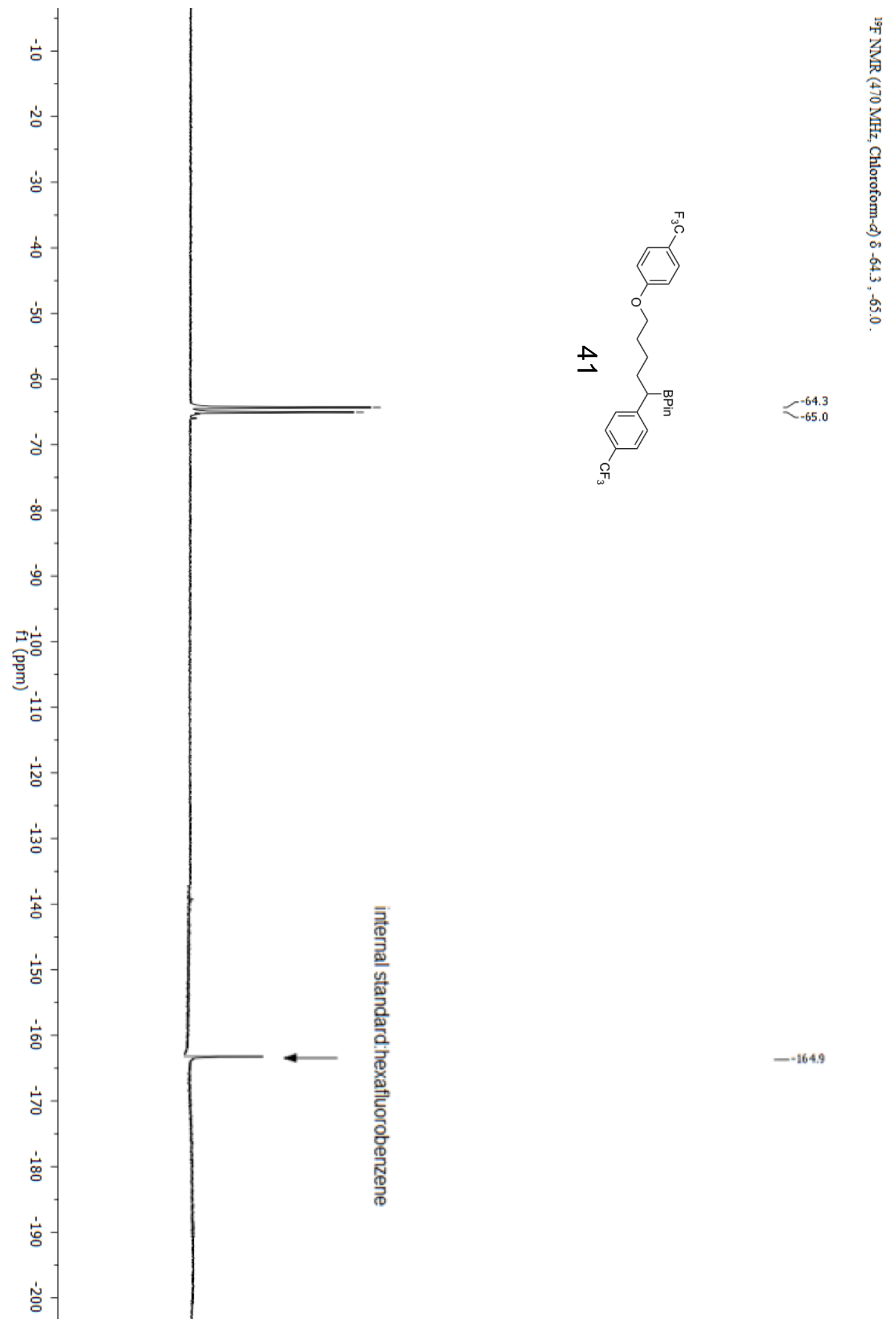

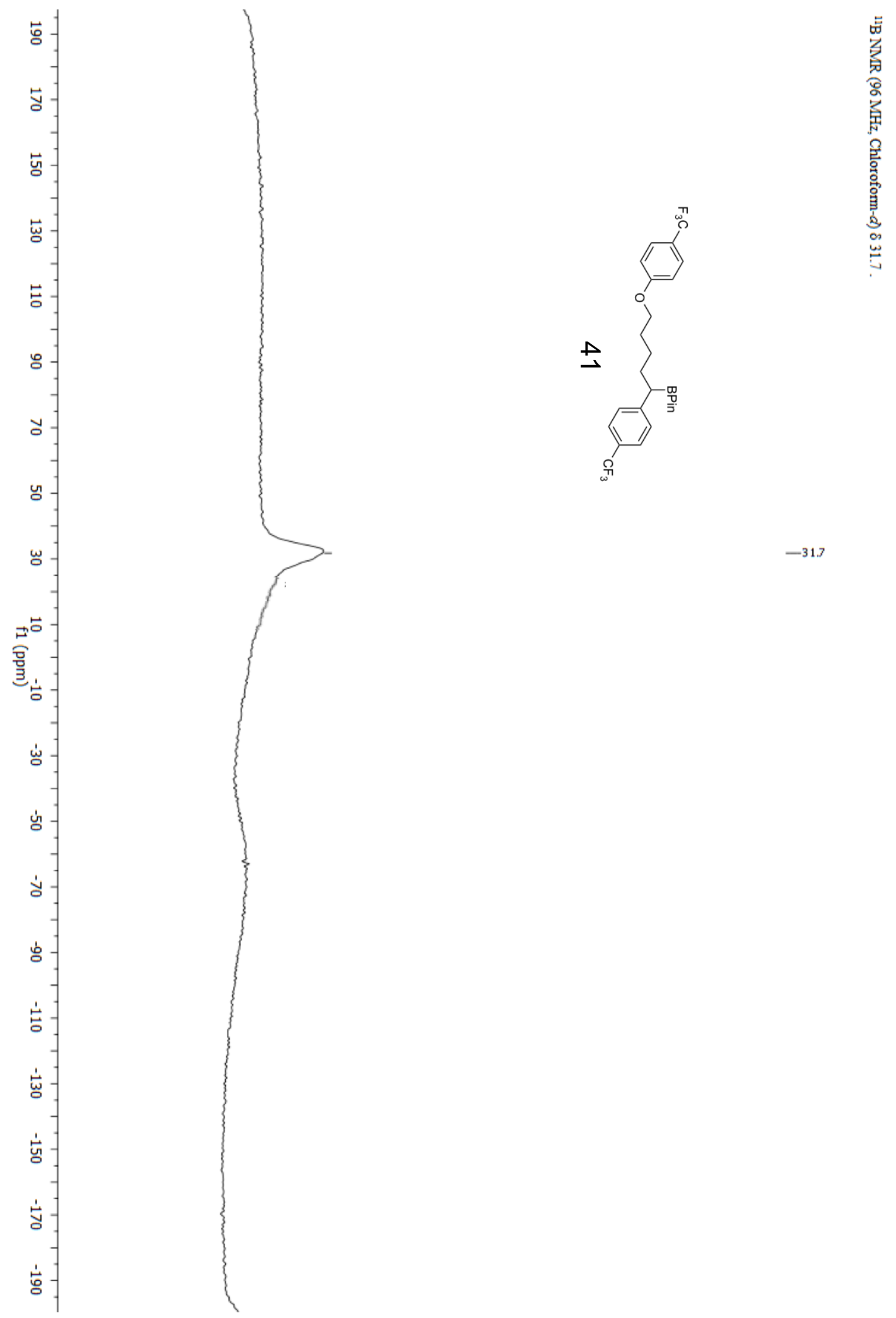


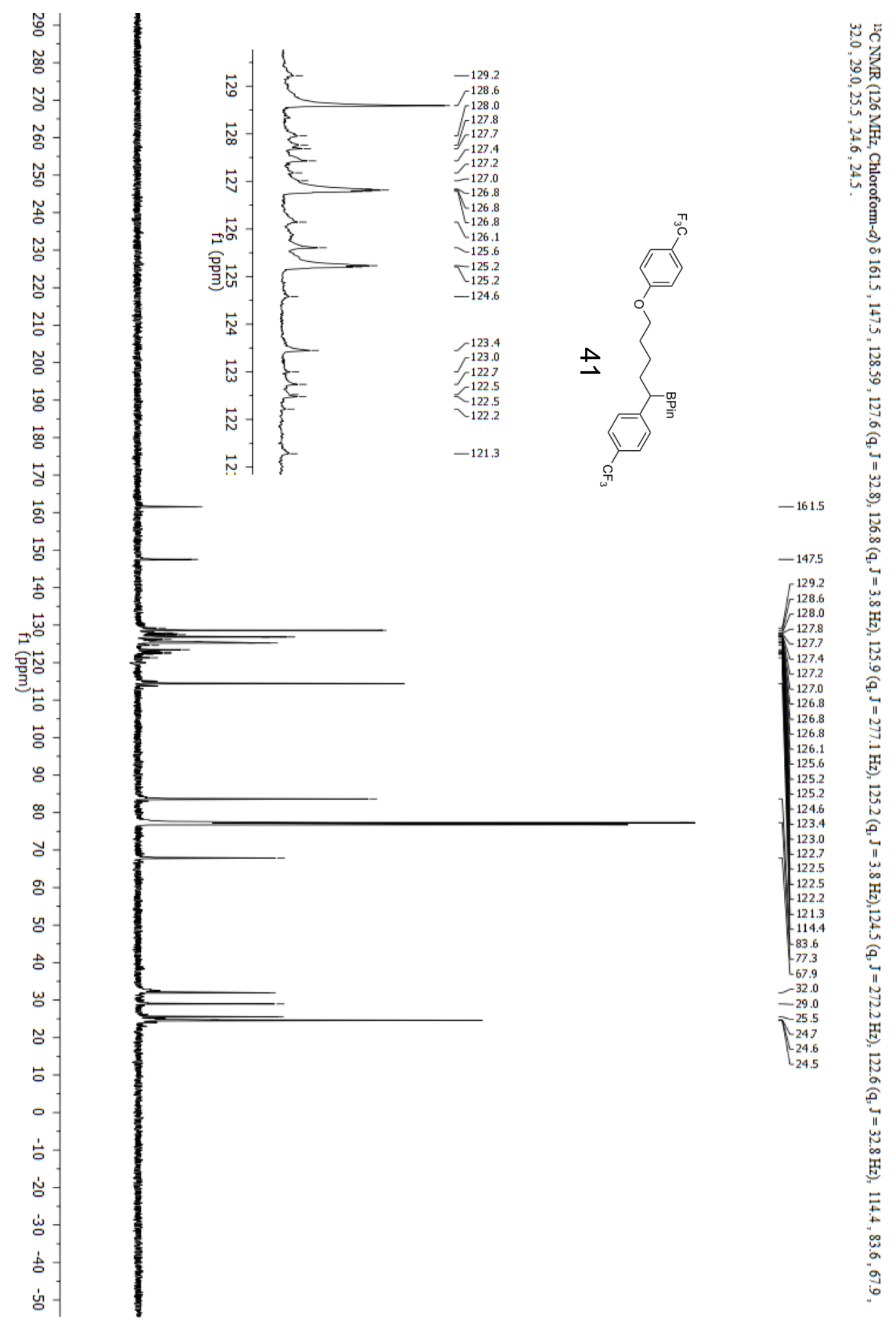



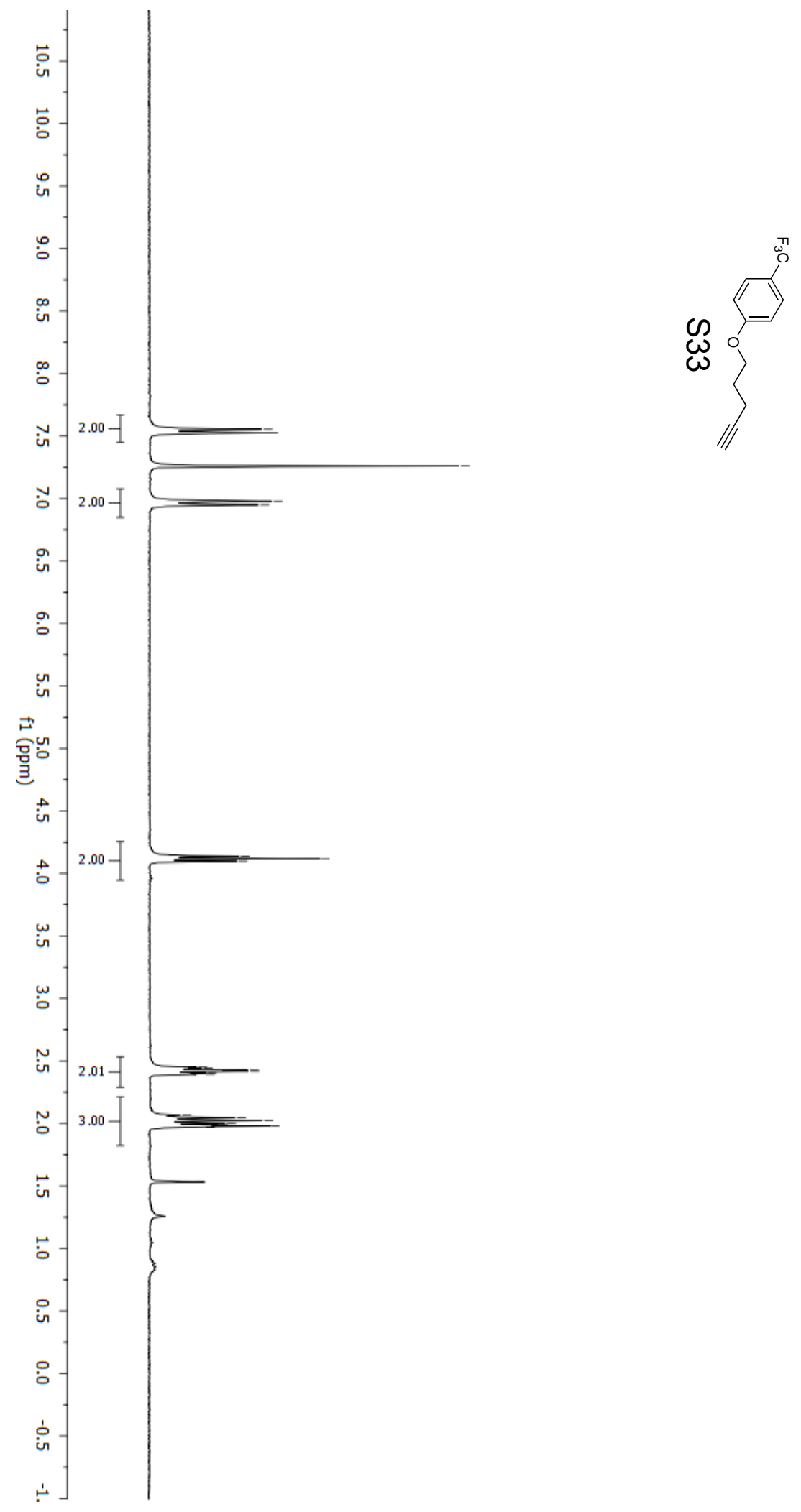

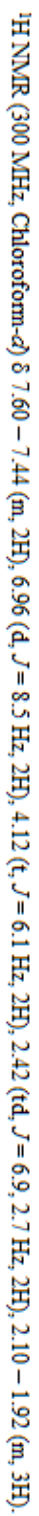




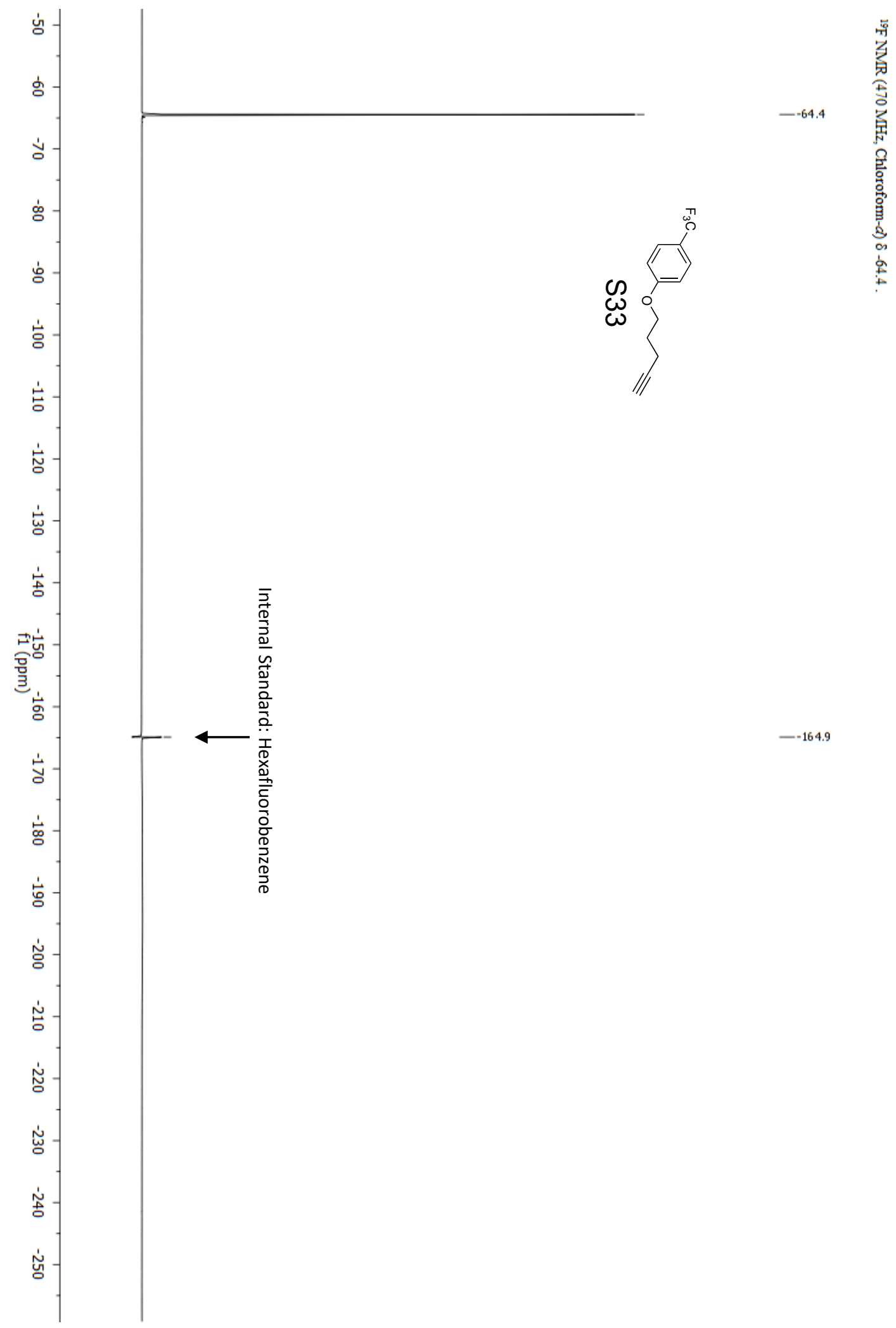




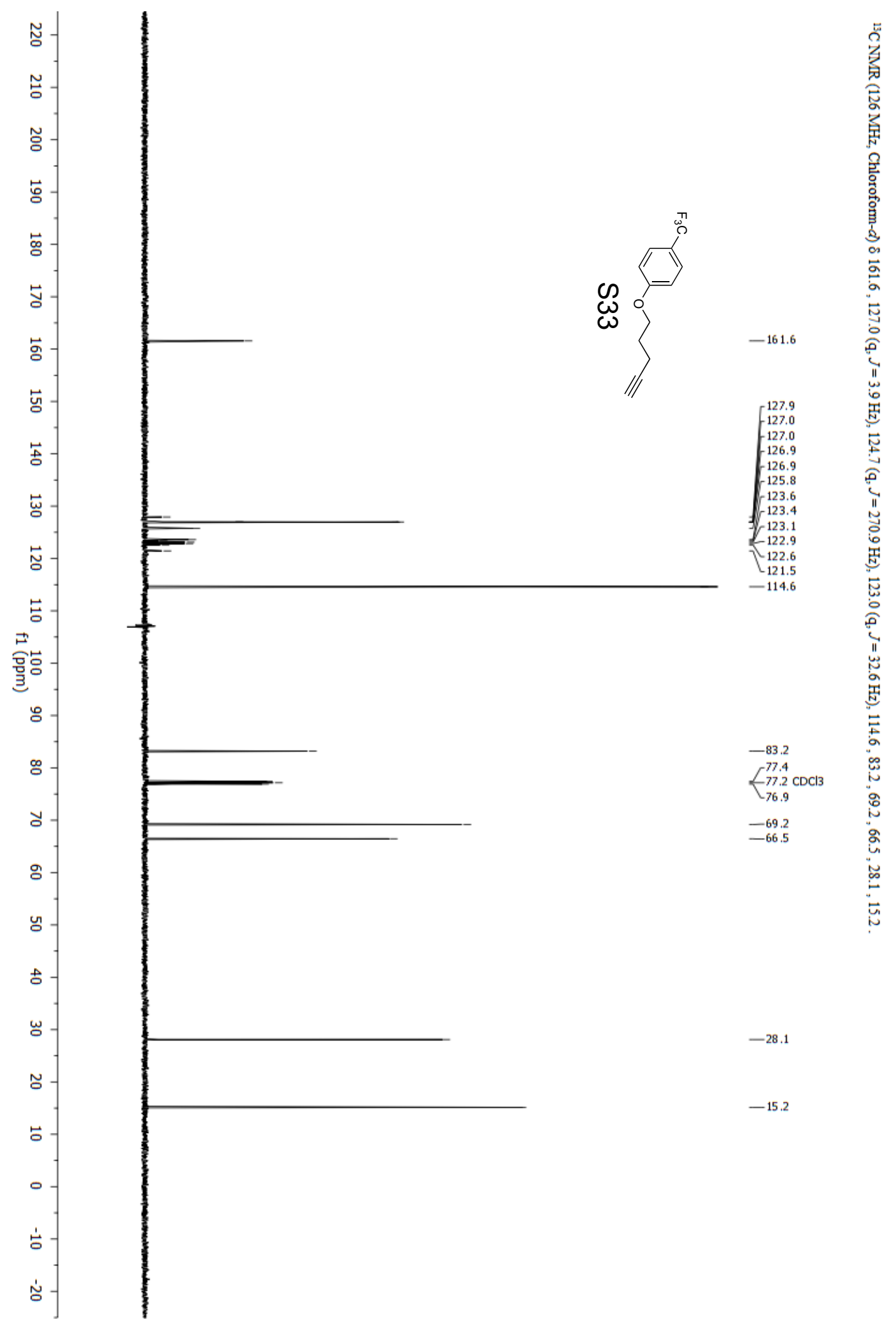




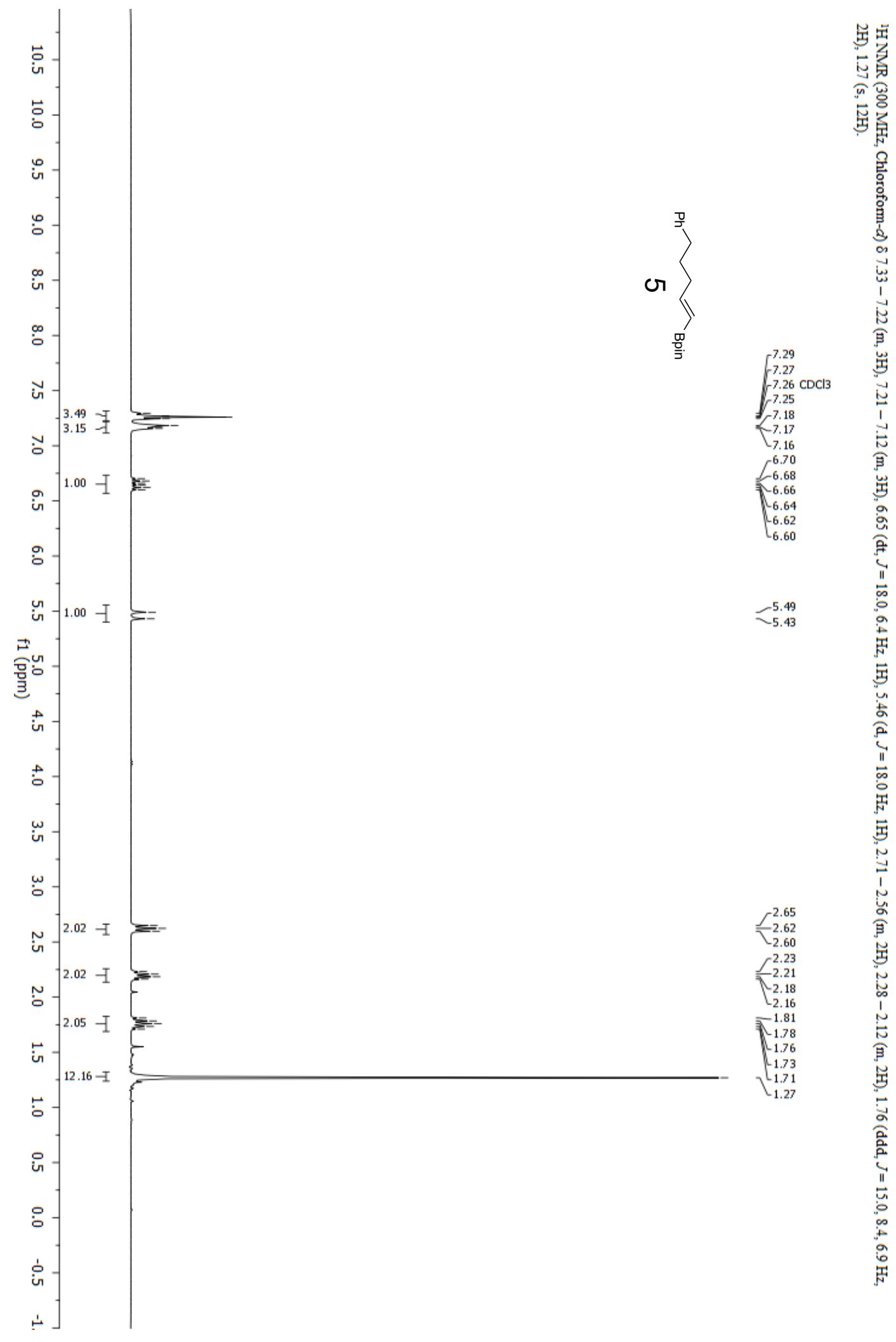



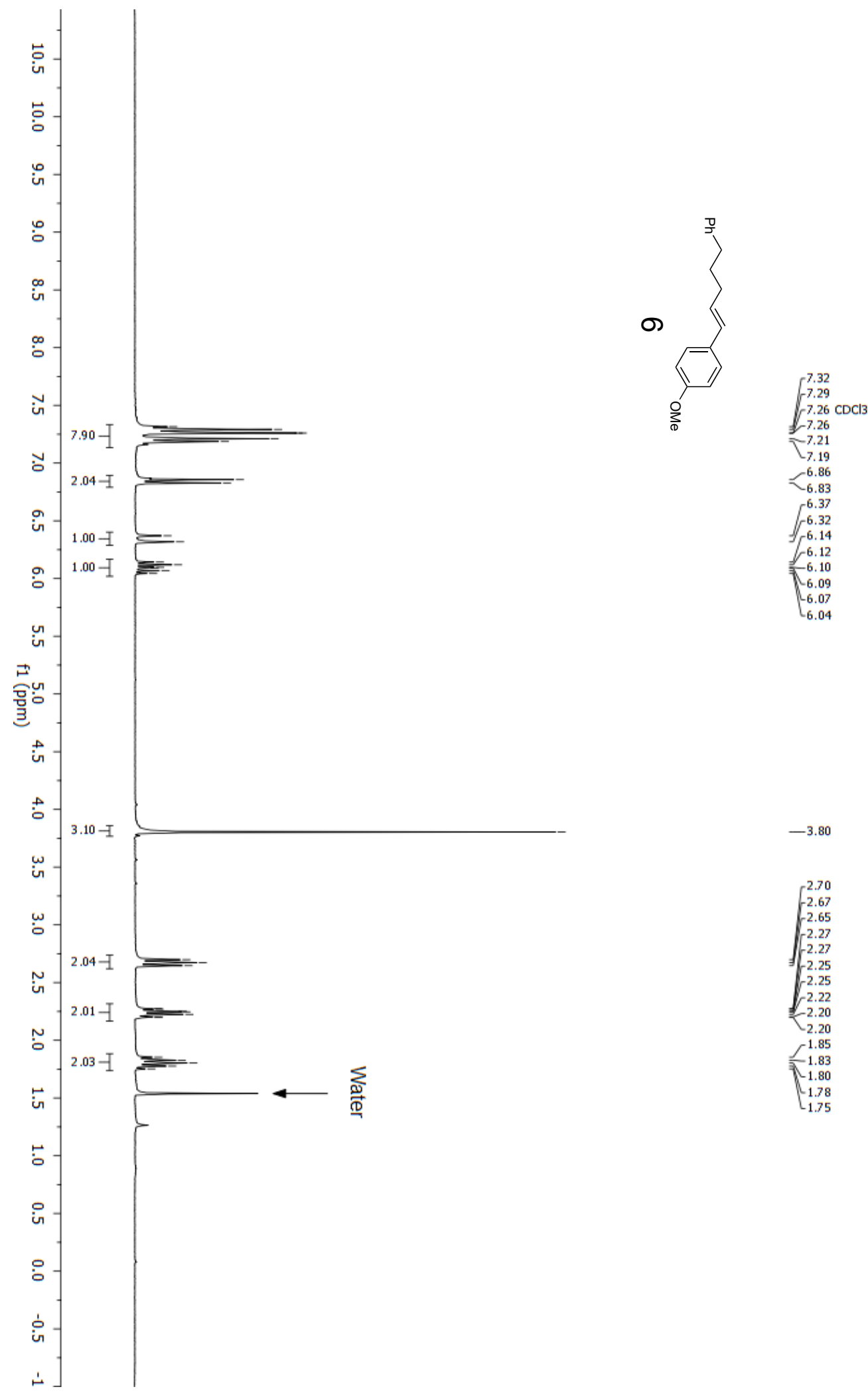

留宙

舀兽

$-3.80$

2.70

$\left\{\begin{array}{l}2.70 \\ -2.67 \\ 2.65 \\ 2.27 \\ 2.27 \\ 2.25 \\ 2.25 \\ 2.22 \\ 2.20 \\ 2.20\end{array}\right.$

$\mathcal{C}_{-1.83}^{1.85}$

$r_{1.80}$

1.75

年 


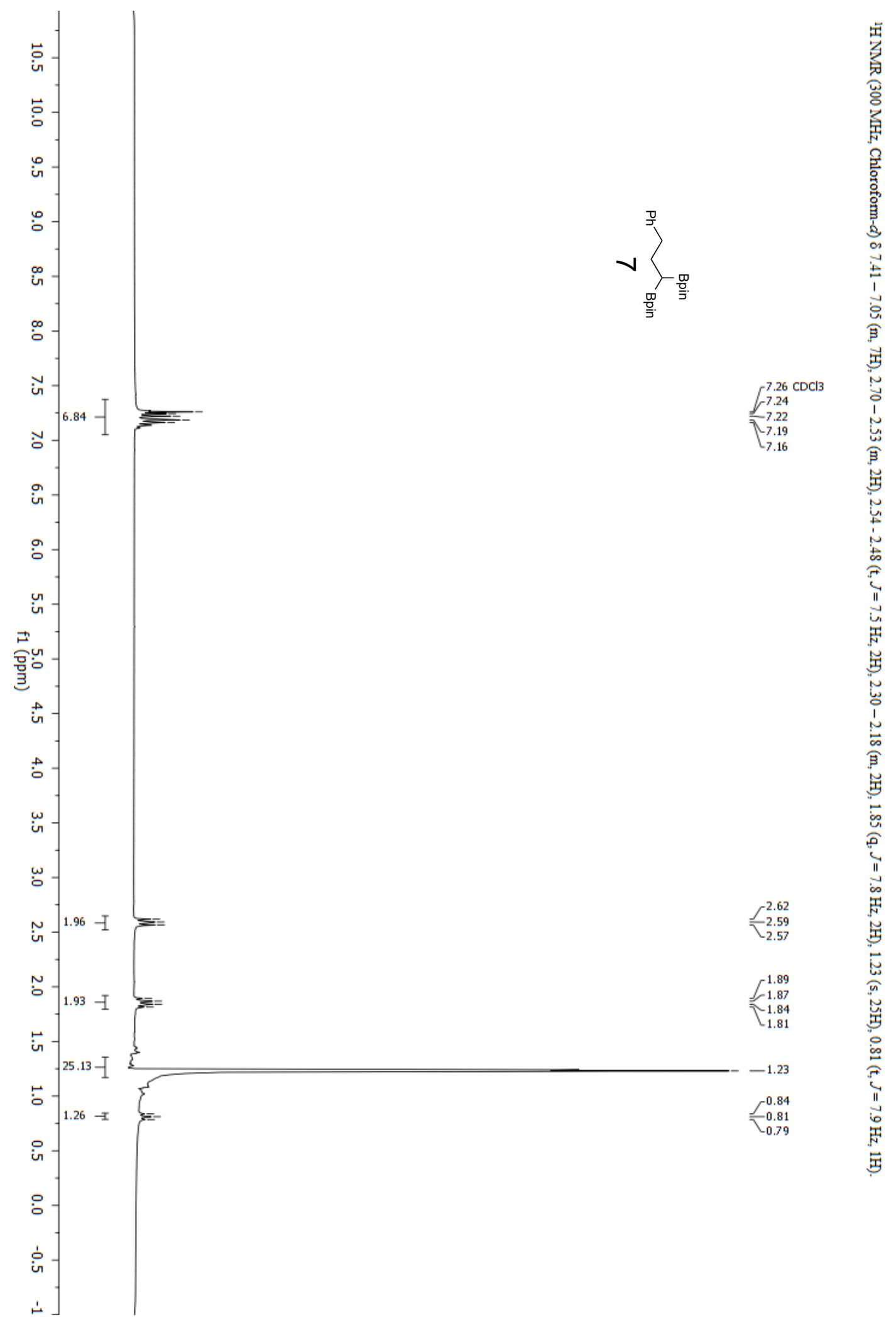



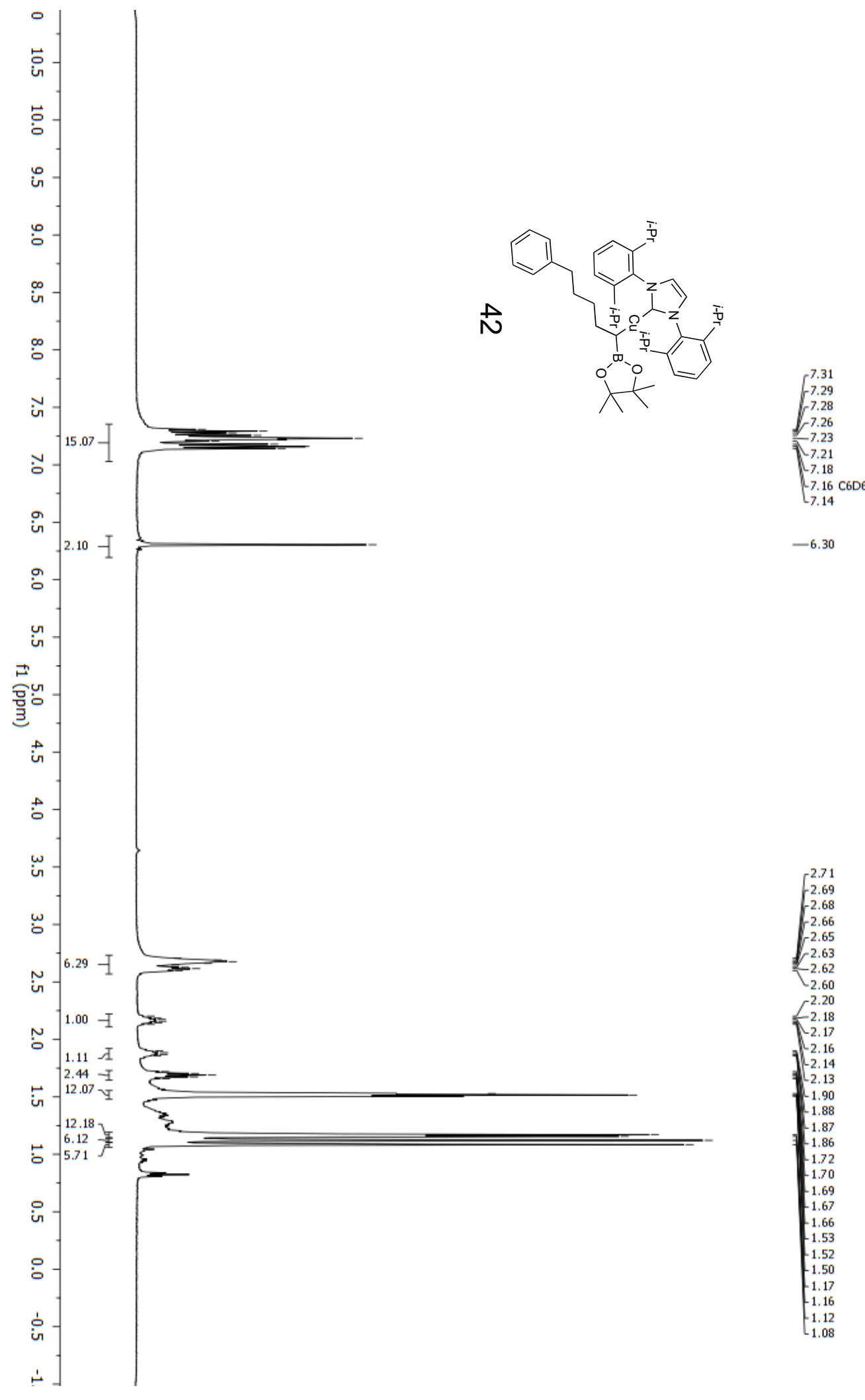

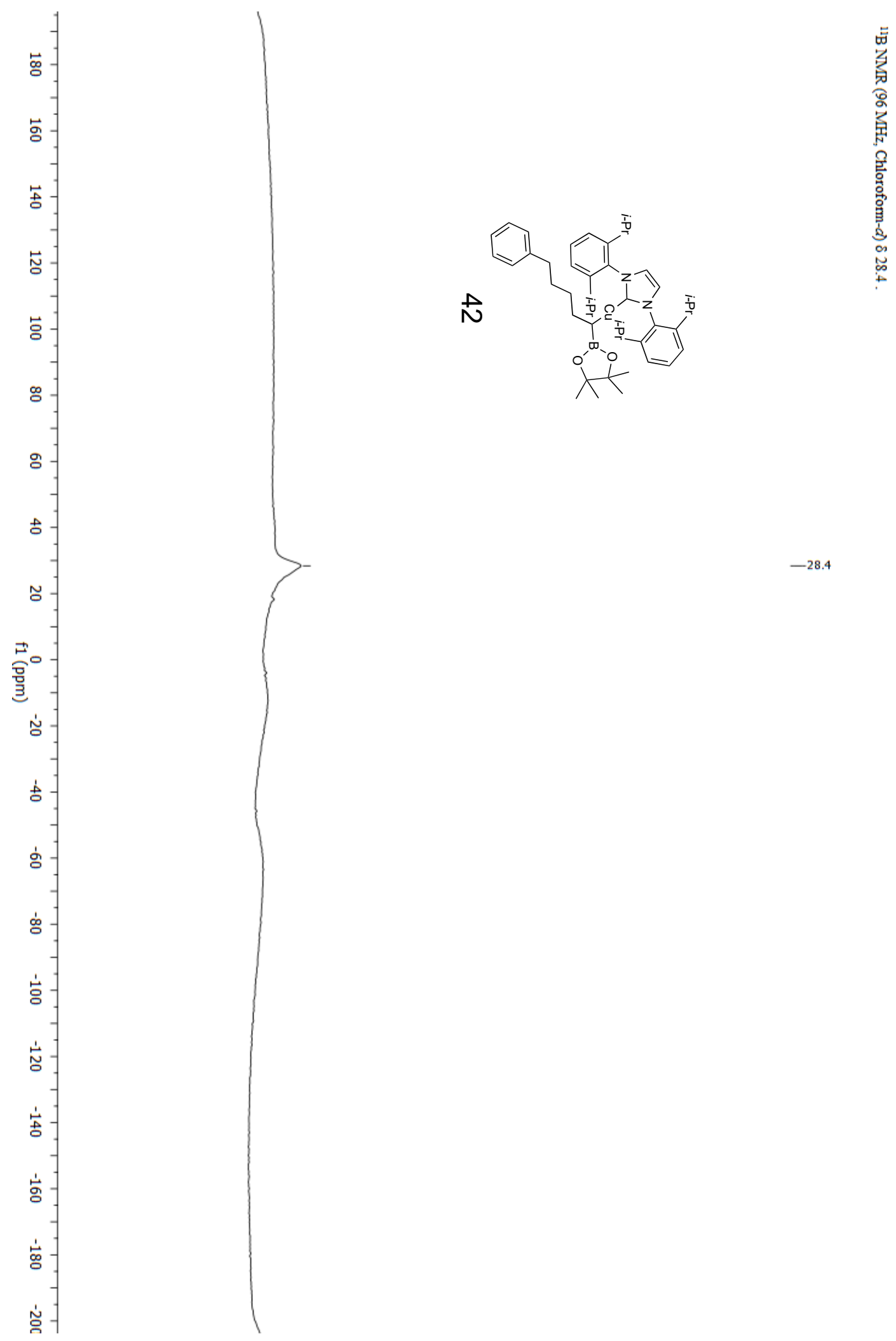

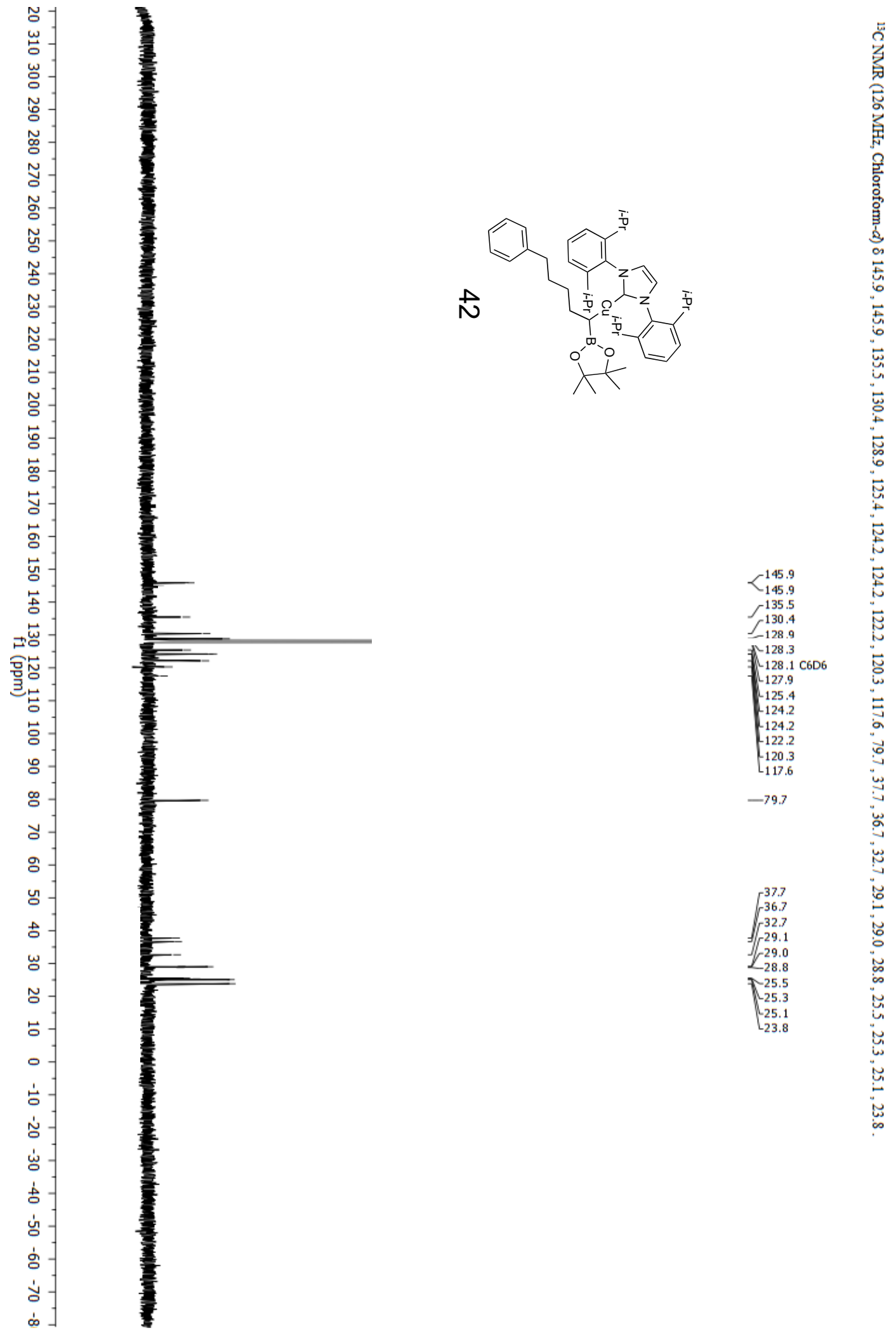

S152 